\title{
Cholesterol metabolism in mouse models of Multiple Sclerosis
}

\author{
Dissertation \\ for the award of the degree \\ "Doctor rerum naturalium" \\ of the Georg-August-Universität Göttingen \\ within the doctoral program Molecular Medicine \\ of the Georg-August University School of Science (GAUSS) \\ submitted by \\ Stefan Alfons Berghoff \\ from Munich
}

Göttingen, 2020 


\section{Thesis Committee}

Dr. Gesine Saher ( $1^{\text {st }}$ Reviewer $)$

Department of Neurogenetics

Max-Planck-Institute of Experimental Medicine

Prof. Dr. Christine Stadelmann-Nessler ( $2^{\text {nd }}$ Reviewer)

Institute for Neuropathology

University Medical Center

Prof. Dr. Alexander Flügel

Institute for Neuroimmunology and Multiple Sclerosis Research

University Medical Center

\section{Further members of the Examination Board}

Prof. Dr. Dr. Hannelore Ehrenreich

Clinical Neuroscience

Max-Planck-Institute of Experimental Medicine

Prof. Dr. Till Ischebeck

Department of Plant Biochemistry

Albrecht-von-Haller-Institute

Prof. Dr. Tiago Fleming Outeiro

Department of Experimental Neurodegeneration

University Medical Center

Date of oral examination: 02.03 .2020 


\section{Declaration}

I hereby declare that the Ph. D. thesis entitled, "Cholesterol metabolism in mouse models of Multiple Sclerosis", was written independently and with no other sources and aids than quoted.

Göttingen, 15.01.2020

Stefan Alfons Berghoff 


\section{Table of content}

List of abbreviations......................................................................

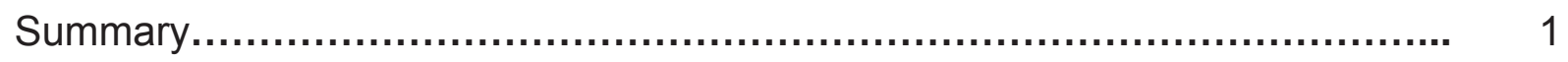

1. Introduction....................................................................... 2

$1.1 \quad$ Cholesterol in the CNS..................................................... 2

1.1.1 Cholesterol synthesis pathway.................................... 2

1.1.2 Regulation of cholesterol metabolism........................... 6

1.1.3 Cholesterol transport in the CNS................................... 8

1.1.4 Neuronal and glial contributions to brain cholesterol........... 10

$1.2 \quad$ Blood-brain barrier........................................................... 13

1.2.1 The Neurovascular Unit, Tight junctions and CNS transport 13

1.2.2 Blood-brain barrier during neuroinflammation.................. 17

$1.3 \quad$ Multiple sclerosis............................................................... 19

1.3.1 Multiple Sclerosis Pathology..................................... 20

1.3.2 Remyelination in Multiple Sclerosis............................. 22

1.3.3 Animal models in Multiple Sclerosis research................... 24

2. Publications/Manuscripts.......................................................... 30

2.1 Publication 1: Dietary cholesterol promotes repair of demyelinated lesions in the adult brain (Berghoff et al., 2017a)........................ 30

2.2 Publication 2: Blood-brain barrier hyperpermeability precedes demyelination in the cuprizone model (Berghoff et al., 2017b)....... 58

2.3 Manuscript: Phase and cell-type specific post-squalene sterol synthesis coordinates inflammation and remyelination................. 76

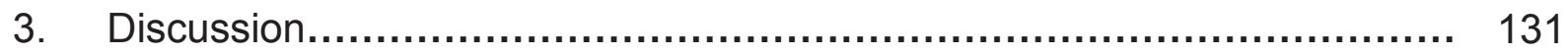

4. References................................................................... 136

5. Acknowledgements........................................................... 160

6. Publications and presentations................................................... 162

7. Own contribution............................................................... 164

8. Curriculum vitae....................................................................... 173 


\section{Abbreviations}

\section{ABCA1}

AIF1

ApoE

AQP4

BBB

BEC

CAll

CC

cKO

Cldn5

CNS

CX3CR1

CXCR3

DHCR24

DMHCA

EAE

FDFT1

FGF

GFAP

GFP

HMGCR

i.p.

i.v.

IFN $\beta-1 b$

IIDD

II1b

LDLR

LPS

LXR
ATP Binding Cassette Subfamily A Member 1

Allograft Inflammatory Factor 1 (Iba1)

Apolipoprotein E

Aquaporin 4

Blood-brain barrier

Brain Endothelial Cell

Carbonic Anhydrase II

Corpus Callosum

Conditional Knockout

Claudin 5

Central Nervous System

C-X3-C Motif Chemokine Receptor 1

C-X-C Motif Chemokine Receptor 3

24-Dehydrocholesterol Reductase

$\mathrm{N}, \mathrm{N}$-dimethyl-3ß-hydroxycholenamide

Experimental Autoimmune Encephalomyelitis

Farnesyl-Diphosphate Farnesyltransferase 1

Fibroblast Growth Factor

Glial Fibrillary Acidic Protein

Green Fluorescent Protein

HMG-CoA reductase

Intraperitoneal

Intravenous

Interferon beta

Idiopathic Inflammatory Demyelinating Disorder

Interleukin 1 beta

Low Density Lipoprotein Receptor

Lipopolysaccharide

Liver X Receptor 
Mac3

MOG

MRI

mRNA

MS

NOS2

NVU

Ocln

$\mathrm{OL}$

OLIG2

OPC

qPCR

scRNA seq.

SEM

SQS

TC

Tnf

$\mathrm{v} / \mathrm{v}$

$w / v$

wt

ZO-1

Lysosome-associated membrane protein 2 (CD107b)

Myelin Oligodendrocyte Glycoprotein

Magnetic Resonance Imaging

Messenger Ribonucleic acid

Multiple Sclerosis

Nitric Oxide Synthase 2

Neurovascular Unit

Occludin

Oligodendrocyte

Oligodendrocyte Transcription Factor 2

Oligodendrocyte Precursor cell

Quantitative polymerase chain reaction

Single-cell RNA sequencing

Standard error of the mean

Squalene Synthase

Total Cholesterol

Tumor Necrosis Factor

Volume percent

Weight per volume

Wildtype

Zonula Occludens 1 


\section{Summary}

The primary origin of brain cholesterol is de novo synthesis due to limited entry peripheral cholesterol across the blood-brain barrier (BBB). Most if not all brain cells contribute to central nervous system (CNS) cholesterol homeostasis. During development, oligodendrocytes produce the majority of cholesterol which is ratelimiting for myelin biogenesis while in the adult brain the role of individual cell-types in cholesterol homeostasis is not well-known. In demyelinating diseases such as multiple sclerosis (MS), CNS cholesterol homeostasis is disturbed likely contributing to disease. However, limited numbers of studies have addressed the relationship between cholesterol availability, cholesterol synthesis, inflammation and remyelination. In this study, I could demonstrate that the disruption of the BBB in the cuprizone intoxication model of de- and remyelination allows for entry of peripherally supplemented cholesterol which supports oligodendrocyte precursor cell (OPC) proliferation and differentiation, restores the balance of growth factors and thereby creates a permissive environment for remyelination. Further, I could show that persistent BBB impairment precedes demyelination characterized by induction of inflammatory mediators mainly of astroglial origin. In addition, I assessed the contribution of individual cell-types to CNS cholesterol metabolism during remyelination. Therefore, cholesterol metabolism during remyelination by tissue based and cell type specific sterol gene expression profiling was comprehensively analyzed and remyelination efficiency of cell type specific conditional cholesterol synthesis mutants during acute and chronic remyelination evaluated. Here, it could be shown that (I) chronic remyelination is driven by the capability of oligodendroglia to synthesize cholesterol, (II) cholesterol synthesis ablation in phagocytes prevents accumulation of the Liver $X$ receptor (LXR) ligand desmosterol leading to chronic activation and lipid export deficiency thereby preventing remyelination, (III) exogenous cholesterol precursor therapy reduces disease severity by inducing LXR activation through increased abundance of desmosterol suppressing inflammation, in addition to directly supporting myelination in oligodendroglia and (IV) cholesterol precursor administration can be integrated in combination therapy. Together, my studies shed light on cholesterol metabolism during remyelination and provide evidence for lipid based therapy in promoting repair after demyelinating episodes. 


\section{Introduction}

\subsection{Cholesterol in the CNS}

The brain is the most cholesterol rich organ and harbors around $20-25 \%$ of the total amount of body cholesterol (Bjorkhem and Meaney, 2004). Within the brain, 70 to $80 \%$ of cholesterol is located in myelin that is formed by oligodendrocytes and insulates axons to facilitate fast nerve conduction velocity (Saher et al., 2011). Cholesterol metabolism in the CNS is essentially independent from the periphery due to the blood brain barrier (BBB), which is established around embryonic day 14 in mice (Hagan and Ben-Zvi, 2015). Hence, most of the brain cholesterol is synthesized locally by de novo synthesis, a complex and resource-intense process with highest rates of about $250 \mu \mathrm{g}$ per day during postnatal myelination (Dietschy, 2009). Following brain maturation cholesterol synthesis remains at a low stable production rate of 25-35 $\mu \mathrm{g}$ per day with a low turnover rate, resulting in a half-life of around 6 month in rodents and up to 5 years in humans (Bjorkhem et al., 1998; Dietschy, 2009). Most if not all brain cells contribute to the CNS cholesterol homeostasis by cell autonomous synthesis. Importance of physiologic brain cholesterol metabolism is highlighted by defects related to cholesterol synthesis and processing pathways leading to maturation defects and life threatening disorders (Bjorkhem et al., 2010; Dietschy, 2009; Martin et al., 2014; Orth and Bellosta, 2012; Saher and Stumpf, 2015; Segatto et al., 2019). In addition, several studies have linked pathology in neurodegenerative diseases to altered brain cholesterol metabolism. However, in the adult brain and during neurodegeneration, the cell type specific extent of cholesterol synthesis still remains largely unknown.

\subsubsection{Cholesterol synthesis pathway}

Cholesterol synthesis is an energy expensive anabolic process consuming 18 ATP and 25 reduction equivalents (e.g. NADPH) that involves over 20 enzymes (Cerqueira et al., 2016; Kovacs et al., 2007; Liscum, 2002; Mazein et al., 2013; Saher et al., 2011). In all mammalian nucleated cells synthesis of the 27 carbon compound cholesterol starts from acetyl-CoA that is generated by cytoplasmic, mitochondrial, or peroxisomal oxidation (Chakrabarti et al., 2017) (Figure 1). HMG-CoA synthase (HMGCS) encoded by cytosolic Hmgcs1, catalyze the irreversible condensation of 
acetyl-CoA and acetoacetyl-CoA to form 3-hydroxy-3-methylglutaryl-CoA (HMG-CoA) which is the first reaction of the isoprenoid biosynthesis pathway. HMG-CoA dictated to cholesterol synthesis is mainly formed by cytosolic HMGCS1, although functional relevance for sterol synthesis by HMGCS1 in peroxisomes has been suggested (Faust and Kovacs, 2014; Olivier and Krisans, 2000). Mitochondrial HMGCS2 is the rate-limiting enzyme for ketogenesis in liver cells that provides fuel for extrahepatic tissues (Grabacka et al., 2016; Kovacs et al., 2007). However, mitochondrial HMGCS2 mediated cholesterol-convertible HMG-CoA production could be shown in cell culture (Ortiz et al., 1994). Rate-limiting for sterol synthesis is the conversion of HMG-CoA to mevalonate by HMG-CoA reductase (encoded by Hmgcr), mainly anchored to the endoplasmic reticulum or in lesser abundance to peroxisomes (Breitling and Krisans, 2002; Kovacs et al., 2007). HMG-CoA reductase is one of the most regulated enzymes and can be targeted by competitive inhibitors (statins) which have become a breakthrough in the management of dyslipidemia (Endo, 2010; Goldstein and Brown, 1990). Following mevalonate synthesis, ATP dependent conversion by mevalonate kinase (MVK) leads to the formation of mevalonate 5phosphate that is further converted to the isoprenoid geranylgeranyl pyrophosphate (Geranyl-PP) via Phosphomevalonate kinase (PMVK), Diphosphomevalonate decarboxylase (MVD), Isopentenyl-diphosphate delta-isomerases (IDI1, IDI2) and Geranylgeranyl pyrophosphate synthase (GGPS1) or Farnesyl diphosphate synthase (FDPS). GGPS1 and FDPS catalyze two reactions that ultimately lead to the formation of the isoprenoid farnesyl diphosphate. Synthesized pre-squalene isoprenoid precursors (isoprenoid lipids) are important for post-translational modification of proteins (protein prenylation), facilitating protein-protein interaction, protein stability and thereby localization of proteins to membranes e.g. small GTPbinding proteins (Park et al., 2014; Wang and Casey, 2016). In addition, FDPS leads to the formation of essential precursors for synthesis of dolchiols and ubiquinone (Coenzym Q10) (Cantagrel and Lefeber, 2011; Faust et al., 1979) (Figure 1). Of note, there is conflict about the subcellular localization of enzymes converting mevalonate to farnesyl pyrophosphate (Farnesyl-PP). Although data suggest peroxisomal localization, cytosolic localization especially for the three ATP dependent enzymes MVK, PMVK and MPD are established (Biardi et al., 1994; Hogenboom et al., 2004a, b; Kovacs et al., 2007; Wang et al., 2016b). Giving the possibility of shared 
localization, loss of PEX5 in mice leading to absence of functional peroxisomes, does not affect cholesterol synthesis (Hogenboom et al., 2002). The first committed step to cholesterol synthesis is catalyzed by farnesyl-diphosphate farnesyltransferase 1 (FDFT1, Squalene synthase) forming the 30 carbon terpene squalene by two-step dimerization of two farnesyl diphosphate molecules. FDFT1 is highly regulated and the promoter region of the encoding gene harbors multiple binding sites for sterol regulatory element-binding proteins (SREBPs). Targeting FDFT1 has been suggested as alternative lipid lowering strategy, circumventing side effects induced by blocked synthesis of isoprenoid precursors by inhibiting HMG-CoA reductase (Charlton-Menys and Durrington, 2007). To date, only one FDFT1 inhibitor has been tested in humans and failed in clinical trials, due to hepatic safety issues (Stein et al., 2011). By two-step cyclization via squalene epoxidase (SQLE) and lanosterol synthase (LSS), squalene is converted to lanosterol which is a branching point of the cholesterol biosynthesis pathway (Buhaescu and Izzedine, 2007). Conversion of lanosterol to the final product cholesterol is a complex multistep process (Figure 1). Two pathways separated by the intermediate that undergoes C24 double bound side chain reduction via 24-dehydrocholesterol reductase (DHCR24), have been proposed. Therefore, conversion of lanosterol by lanosterol 14 alpha-demethylase (CYP51) is termed Bloch pathway, forming a series of intermediates with unsaturated side-chain resulting in the final reduction of the intermediate desmosterol to cholesterol (Bloch, 1965). Alternatively, the Kandutsch-Russel pathway has been proposed, starting with formation of dihydrolanosterol via DHCR24 mediated reduction of lanosterol, creating several intermediates with saturated side chain and proceeding by the final conversion of 7-dehydrocholesterol to cholesterol through 7dehydrocholesterol reductase (DHCR7) (Kandutsch and Russell, 1960a, b). Importantly, recent studies support tissue and cell-type specific use of these two pathways or even alternative branching points. Therefore, within brain tissue a hybrid pathway termed modified Kandutsch-Russel pathway has been proposed starting from side-chain reduction of zymosterol (Mitsche et al., 2015) (Figure 1). However, cholesterol formation from lanosterol requires the terminal cholesterol synthesis enzymes DHCR7 and DHCR24 that potentially even interact forming a "cholesterol metabolon" (Luu et al., 2015). 
Sterol synthesis pathway

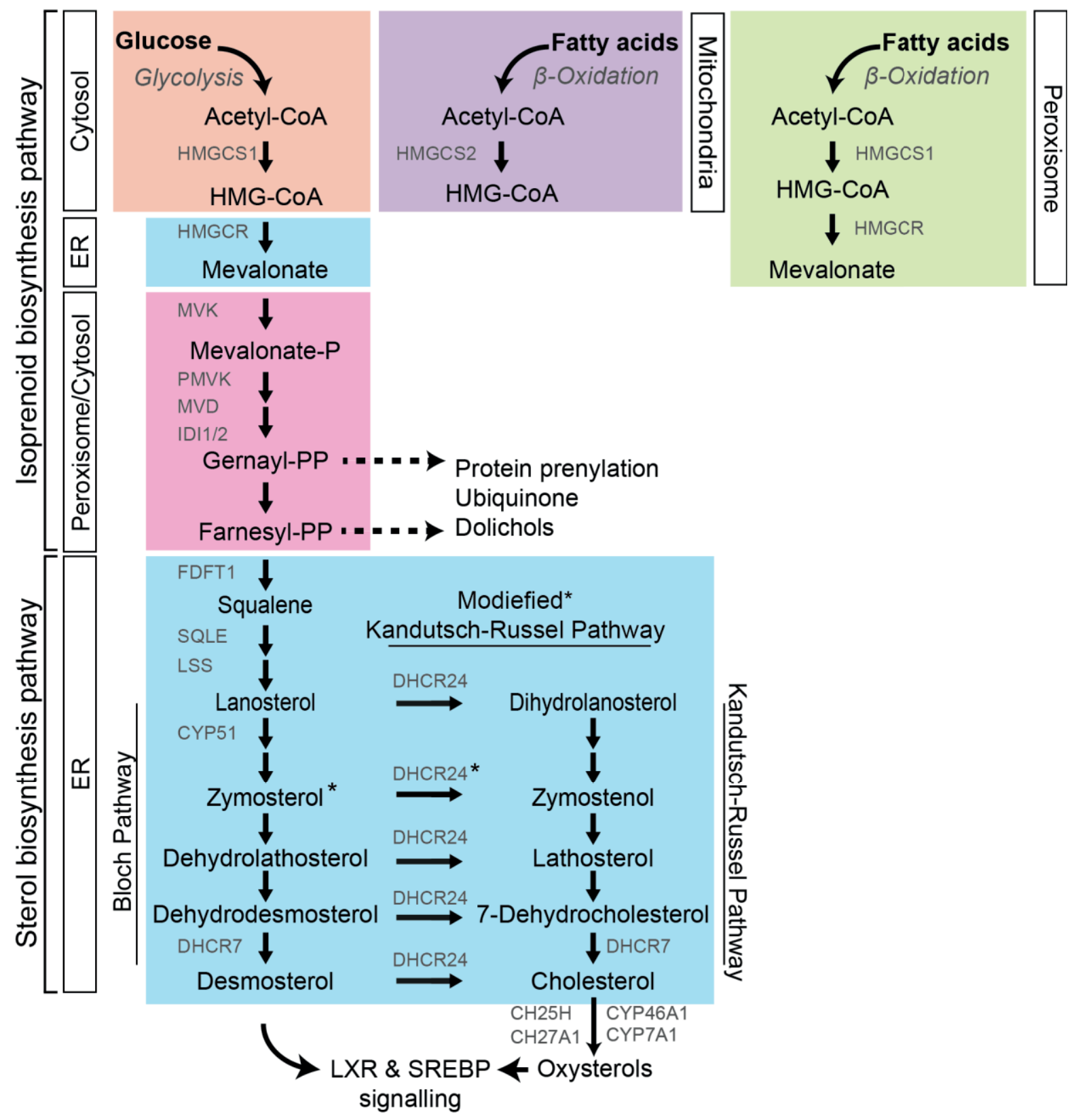

Figure 1: Schematic representation of cholesterol synthesis pathway.

Diagram depicting most of the enzymes involved in isoprenoid and sterol biosynthesis and their intracellular localization (colors). Isoprenoid lipids important for post-translation protein modification and generation of dolichols and ubiquinone are highlighted. Bloch, Kandutsch-Russell and modiefied Kandutsch-Russell pathway for the enzymatic conversion of squalene to cholesterol are indicated (sterol synthesis pathway) as well as enzymes mediating oxysterol synthesis and the LXR ligand function of desmosterol both involved in regulation of cholesterol metabolism. 


\subsubsection{Regulation of cholesterol metabolism}

End-product inhibition of cholesterol synthesis has been already described in 1933 (Schoenheimer and Breusch, 1933). Since then, a complex regulatory machinery not only targeting the rate-limiting enzyme HMG-CoA reductase but also other enzymes of the cholesterol synthesis pathways have been described. Cholesterol metabolism is controlled by several mechanisms including regulation of mRNA transcripts, protein degradation and enzyme activity of important cholesterol homeostasis proteins (Luo et al., 2019). For instance, this combination can impact several hundred fold differences on protein abundance of HMG-CoA reductase (Nakanishi et al., 1988). The identification of a conserved motif regulating HMG-CoA reductase stability termed sterol-sensing domain (SSD) has led to the discovery of several sterol-related proteins (Goldstein et al., 2006; Theesfeld et al., 2011).

One important master regulator controlling sterol synthesis is the sterol regulatory element-binding protein 2 (SREBP-2), an ER membrane anchored inactive transcription factor that binds the SSD harboring SREBP cleavage-activating protein (SCAP) (DeBose-Boyd, 2008; Goldstein et al., 2006; Ye and DeBose-Boyd, 2011) (Figure 2). SREBP-SCAP complex is locked in the ER by the retention proteins INSIG-1 and INSIG-2 (Insulin Induced Gene 1 and 2) that bind to SCAP when ER cholesterol levels exceed $5 \mathrm{~mol} \%$ of total ER lipids (Radhakrishnan et al., 2008). In contrast to SCAP, binding of INSIGs to HMG-CoA reductase induces ubiquitination and degradation. Under low cholesterol conditions, INSIG interaction with SREBP2/SCAP complex is lost leading to coatomer II (COPII) vesicle mediated translocation of the SREBP-2-complex to the Golgi where SREBP-2 undergoes proteolytic cleavage by site-1 protease (S1P) and site-2 protease (S2P). Soluble $\mathrm{NH}_{2}$-terminal fragments are able to enter the nucleus and bind to sterol regulatory elements (Biardi et al., 1994) of target genes inducing expression of several cholesterol synthesis genes (Sharpe and Brown, 2013) (Figure 2). Beside transcriptional control of cholesterol synthesis via SREBP2, a family of transcription factors, namely the Liver $X$ receptors (LXRs) have been implicated as major regulators of cholesterol metabolism (Baranowski, 2008; Courtney and Landreth, 2016; Dietschy, 2009). Of those ligand-activated transcription factors, LXRa (NR1H3) and LXR $(\mathrm{NR} 1 \mathrm{H} 2)$ have been shown to induce expression of genes harboring LXR response elements 


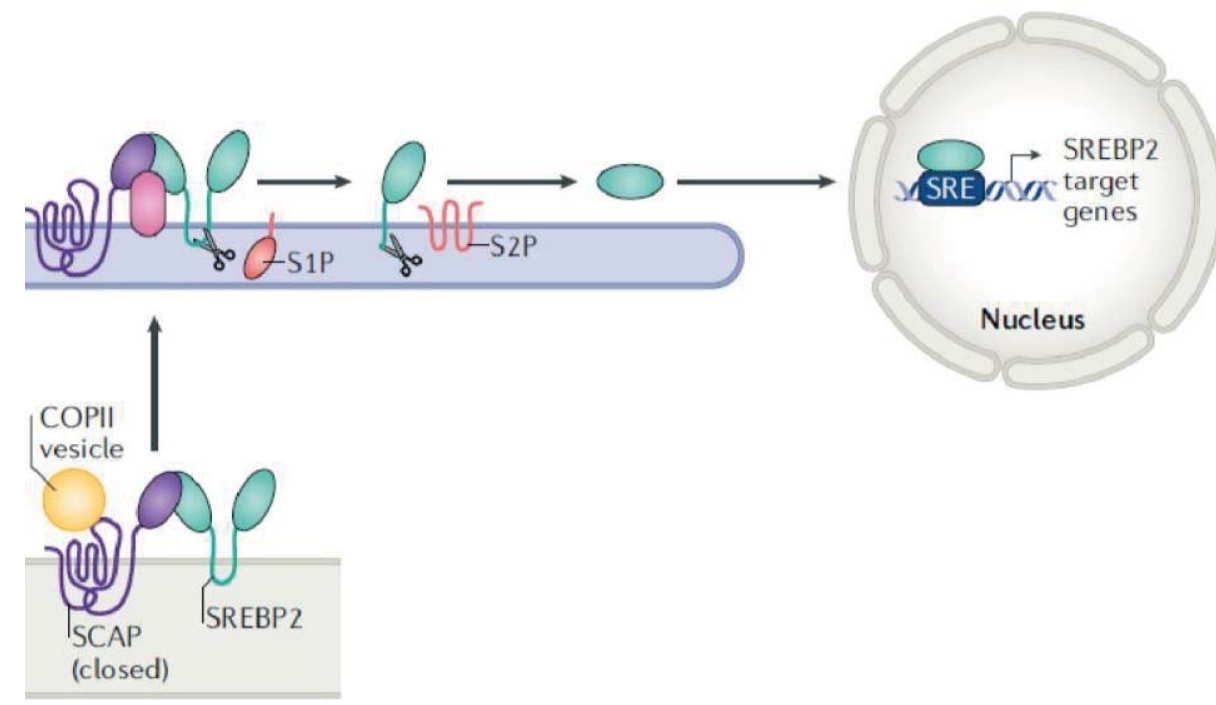

Figure 2. Master sterol synthesis regulator SREBP2.

Sterol regulatory element- binding protein 2 (SREBP2) localized to the ER, interacts with SREBP-cleavage activating protein (SCAP). Under cholesterol starvation, coatomer II (COPII) binds SCAP allowing ER exit of the SCAP-SREBP complex that is anchored to the Golgi. SREBP2 undergoes proteolytic cleavage by site 1 protease (S1P) and S2P in the golgi, releasing the soluble N-terminal domain that enters the nucleus, binds to the sterol regulatory element (Biardi et al., 1994) in the promoter of target genes thereby inducing gene transcription of e.g. cholesterol synthesis genes (modified from Luo et al., 2019).

(LXREs) after heterodimerization with isoforms of the retinoid $X$ receptor (RXR). When cholesterol levels increase, natural LXR ligands are formed by conversion of cholesterol to mono-oxygenated cholesterol derivates (Oxysterols) via several hydroxylases (CYP46A1, CH25H, CYP7b1, CYP27a1) (Figure1) (Huang, 2014). Following ligand binding to LXR, corepressors are released and coactivators recruited (Hu et al., 2003). LXR activation induces expression of genes, regulating cholesterol efflux e.g. ATP binding cassette subfamily A member 1 (ABCA1) (Sparrow et al., 2002) and leads to degradation of the cholesterol uptake low density lipoprotein receptor (LDLR) (Zelcer et al., 2009). In addition, suppression of cholesterol synthesis genes such as FDFT1 and CYP51 (Wang et al., 2008) and SREBP signaling in cell specific manner has been reported (Muse et al., 2018). In addition to oxysterols, the cholesterol intermediate desmosterol exhibits LXR agonist activity, indicating additional regulatory levels of cholesterol metabolism by sterol intermediates (Yang et al., 2006). Cholesterol in the metabolic active pool can alternatively be controlled by esterification via acyl-CoA cholesterol acyltransferase 1 (ACAT1, also named SOAT1) (Chang et al., 2009; Fujimoto and Parton, 2011). In 
cells with excess cholesterol, SOAT 1 mediates formation and lipidation of lipid droplets that emerge from the ER thereby forming a storage organelle for lipids (Olzmann and Carvalho, 2019; Walther and Farese, 2012). Lipid droplets are highly dynamic organelles in close contact with other cellular organelles and can be used as a source for cholesterol by mobilization of cholesterol esters via different lipases e.g. hormone-sensitive lipase (LIPE), hydrolases e.g. neutral cholesterol ester hydrolase (NCEH1) or esterases e.g. carboxylesterase 1 (CES1) (Sakai et al., 2014; Walther and Farese, 2009; Xu et al., 2018).

\subsubsection{Cholesterol transport in the CNS}

Within the CNS, cholesterol is transferred via HDL-like lipoprotein particles composed of a lipid core of esterified cholesterol and triglycerides (TG), surrounded by phospholipids, unesterified cholesterol, and apolipoproteins. The major apolipoprotein forming lipoprotein particles produced in the CNS is apolipoprotein E (ApoE). In cerebrospinal fluid (CSF), distinct lipoprotein classes have been suggested, that are distinguished by their different abundance of apolipoproteins such as ApoE, ApoJ, ApoD, ApoH and ApoA1I/II, their size (10-22nm) and lipidprotein composition (Koch et al., 2001). It has been proposed, that ApoA1 is not synthesized within the brain and transferred from the plasma via scavenger receptor class B type 1 (SR-BI) in addition to being involved in active lipid transfer at the blood-brain barrier (BBB) via phospholipid transfer protein (PLTP) (Balazs et al., 2004; Chirackal Manavalan et al., 2014; Linton et al., 1991). Similar to peripheral cholesterol traffic, CNS lipoprotein metabolism involves classical proteins like lipoprotein lipase (LPL), lecithin-cholesterin-acyltransferase (LCAT), cholesterol ester transfer protein (CETP), ABCA1 and LDL receptor family members. Lipidation of ApoE-containing lipoprotein particles is mediated by ATP-binding cassette (Rabchevsky et al.) transporters like ABCA1 which is essential for normal CNS functioning (Karasinska et al., 2009; Wahrle et al., 2004). Maturation of secreted lipoprotein particles involves remodeling enzymes like LCAT and CETP, responsible for cholesterol esterification and lipid transfer between particles, leading to formation of larger spherical HDL particles (Albers et al., 1992; Vance and Hayashi, 2010). Several receptors, transporters and enzymes mediate cellular uptake of lipoprotein lipids within the CNS (Mahley, 2016; Wang and Eckel, 2014). In the brain, members 
of the LDL receptor family orchestrate receptor-mediated endocytosis of lipid-rich lipoprotein particles. This family consists of several receptors including the wellestablished LDLR that led to the discovery of an internalization and degradation pathway following binding of low density lipoprotein ( $L D L)$ in peripheral tissue (Goldstein and Brown, 2009; Strickland et al., 2002). Incorporation of lipoprotein derived lipids is mediated by LPL facilitating uptake via the LDL receptors and necessary for TG hydrolysis (Loeffler et al., 2007).

Within the CNS several members of the LDL receptor family like LDLR, very low density lipoprotein receptor (VLDLR) and low density lipoprotein receptor related protein 1 (LRP1) have been described with important functions for normal brain development (Zhao et al., 2007). In addition, members of the scavenger receptors SR-BI, SR-BII and SR-BIII (CD36) as well as the ApoB-receptor (APOBR) have been identified. Interestingly, lipoprotein receptors are also involved in signaling pathways independent of lipid uptake. For instance, reelin induces downstream signaling by binding to the VLDLR and APOE receptor 2 (APOER2) and interference impacts neuronal development of the cerebral cortex (Herz and Chen, 2006; Trommsdorff et al., 1999). Following lipid receptor mediated endocytosis, lipid receptors are recycled to the plasma membrane and lipoprotein particles shuttle to the endo/lysosome where cholesterol esters are hydrolyzed (Brown and Goldstein, 1979; Fazio et al., 2000). Two cholesterol binding proteins, Niemann-Pick, Type C1 (NPC1) and NPC2 are critically involved in sorting of endosomal cholesterol (Carstea et al., 1997; Pfeffer, 2019). Genetic mutations of either of these proteins causes Niemann-Pick type C (NPC) a fatal neurodegenerative lysosomal storage disorder most commonly diagnosed during childhood and histopathologically characterized by deposits of lipids, including sphingomyelin, cholesterol, and gangliosides (Carstea et al., 1997; Evans and Hendriksz, 2017). NPC1 and NPC2 are thought to act together in a twostep mechanism, enabling egress of cholesterol from the lysosomal compartment to the ER, plasma membrane or other cell organelles (Chu et al., 2015; Infante et al., 2008; Kanerva et al., 2013; Subramanian and Balch, 2008). In addition, ABCA1 mediated efflux of cholesterol seems to be connected to functional NPC-protein, underlining the importance of these traffic proteins (Boadu and Francis, 2006; Boadu et al., 2012; Subramanian and Balch, 2008). 


\subsubsection{Neuronal and glial contributions to brain cholesterol}

Of the entire pool of unesterified brain cholesterol $70 \%$ locates to myelin, while $20 \%$ are attributed to glia cells and $10 \%$ to neurons (Bjorkhem and Meaney, 2004; Dietschy and Turley, 2004). Astrocytes have been considered to be the major producers of cholesterol in the adult brain (Petrov et al., 2016) (Figure 3) indicated by high basal mRNA transcript levels of cholesterol synthesis genes in vivo (Zhang et al., 2014), being the major brain cell population positive for ApoE-EGFP in transgenic mice (Xu et al., 2006), shuttling cholesterol to neurons in cell culture (Mauch et al., 2001) and rescuing cholesterol deficient neurons by upregulating cholesterol synthesis and transport (Funfschilling et al., 2012). In addition, disruption of astrocyte cholesterol metabolism in Huntington disease limits the supply to neurons thereby contributing to pathology (Valenza et al., 2015). The importance of astrocyte lipid metabolism could be shown, by genetically interfering with lipid and cholesterol synthesis deleting SCAP or SREBP-2 in astrocytes during development (Camargo et al., 2012; Camargo et al., 2017; Ferris et al., 2017; van Deijk et al., 2017). For instance, SCAP deficiency in astrocytes results in impaired brain development and hypomyelination that could be rescued by a lipid enriched diet (Camargo et al., 2012; Camargo et al., 2017). However, SCAP and SREBP-2 signaling is in addition to cholesterol metabolism involved in fatty acid homeostasis (Espenshade, 2006; Ye and DeBose-Boyd, 2011). Therefore, studies specifically targeting cholesterol metabolism in astrocytes are needed to unravel cholesterol contribution not only in the adult brain, but also during development.

It has been shown, that neuronal cholesterol synthesis is indispensable during maturation, although horizontal transfer either by microglia or astrocyte supports cholesterol synthesis deficient neurons (Funfschilling et al., 2012). In addition to that, astrocytes support neurons by transferring ApoE-cholesterol in vitro, although conclusive in vivo data under heathy conditions are missing (Mauch et al., 2001). Although controversial, opposing to astrocytes that seem to synthesize cholesterol via the Bloch pathway indicated by high levels of desmosterol, neuronal cholesterol synthesis has been linked to the Kandusch-Russel pathway (Dietschy, 2009; Nieweg et al., 2009; Pfrieger and Ungerer, 2011). Given the high metabolic rate of neurons, it has been suggested that constant cholesterol turnover via the neuron specific cholesterol-24-hydroxylase (CYP46A1) converting cholesterol to 24S- 
hydroxycholesterol (24OHC) which is able to pass the BBB, accounts for the elimination of the majority of brain cholesterol (Bjorkhem et al., 2019; Dietschy and Turley, 2004; Lund et al., 1999; Xie et al., 2003) (Figure 3). Although direct in vivo evidence of neuronal cholesterol elimination is still missing, correlation of serum 24$\mathrm{OHC}$ levels and neuronal pathology under neurodegenerative conditions may support this model (Bjorkhem et al., 2019; Hughes et al., 2013). Interestingly, cholesterol synthesis as well as $24-\mathrm{OHC}$ conversion seems to be restricted to the neuronal somata and is not found in the narrow ER of axons (Ramirez et al., 2008; Ramirez and Couve, 2011; Terasaki, 2018; Vance et al., 1994). Supporting this, sciatic nerve crush induces an ER generated retrograde injury signal that is transported to the nucleus, initiating axon regeneration by increasing cholesterol synthesis (Ying et al., 2015). During early postnatal development, cholesterol synthesis in oligodendrocytes is rate-limiting for myelination. Loss of cholesterol synthesis in oligodendrocytes seems to be partially compensated by other glial cells but leads to persistent reduction of myelin cholesterol (Saher et al., 2005). During this critical period, CNS cholesterol synthesis rate closely correlates with cholesterol content of the brain. In addition, postnatal oligodendrocytes selectively express LDLR and VLDLR during myelination suggesting additional import of cholesterol from glia cells e.g. astrocytes (Zhao et al., 2007). In the adult brain, oligodendrocytes show second highest mRNA transcript levels of genes related to cholesterol synthesis and transfer (Zhang et al., 2014). Therefore, it has been suggested that oligodendrocytes rely on their own cholesterol synthesis for myelin maintenance although direct in vivo evidence is missing (Martin et al., 2014). The contribution of other glial cells to CNS cholesterol metabolism remains enigmatic. In the adult brain, Microglia express ApoE, likely contributing to horizontal cholesterol transfer (Zhang et al., 2014) (Figure 3). Interestingly, high cholesterol in vitro conditions are essential for microglial survival and homeostatic resting phenotype, indicating that microglia are important sensors for CNS cholesterol homeostasis (Bohlen et al., 2017). Brain endothelial cells (BECs), forming a barrier for CNS lipid entry during healthy conditions, have been suggested to be involved in HDL generation at the brain parenchymal site although direct in vivo evidence is lacking (Andreone et al., 2017; Balazs et al., 2004; Chirackal Manavalan et al., 2014). In addition, these cells might be involved in ABCA1-ApoE mediated cholesterol export from the brain (Dietschy, 2009; Hirsch- 
Reinshagen et al., 2004) (Figure 3). In principle, BECs could be involved in active import of cholesterol into the CNS, which might be relevant during disease conditions (Abbott and Friedman, 2012; Abbott et al., 2010; Stefani and Liguri, 2009). In summary, data from cell-specific knock-out mice indicate the essential need of oligodendroglial, astroglial and neuronal cholesterol synthesis and limitations of horizontal transfer during early development. However, studies targeting cholesterol synthesis and metabolism in the adult brain with cellular specificity are needed to understand cholesterol homeostasis in the mature CNS.

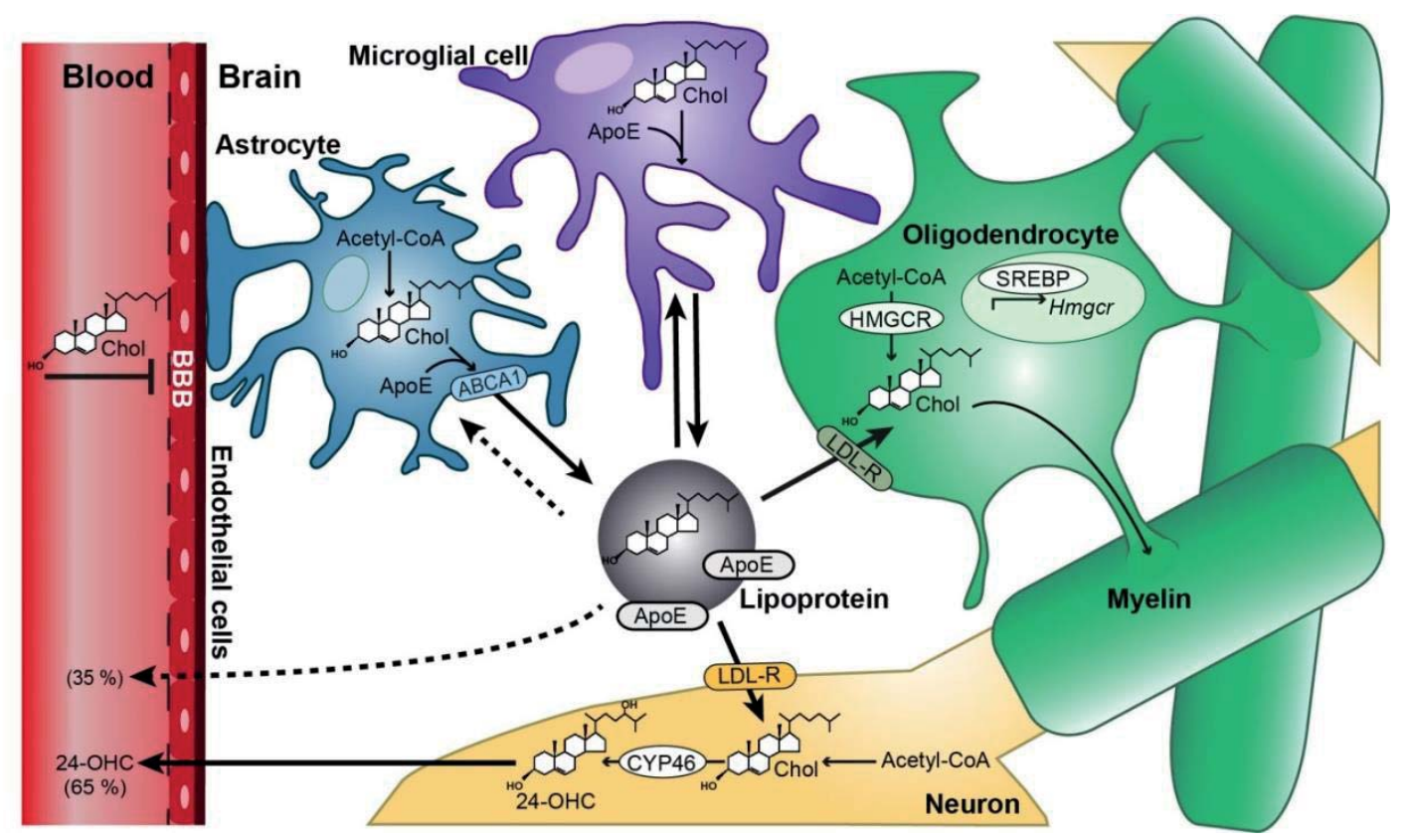

Figure 3: Model of cholesterol metabolism in the CNS

Peripheral cholesterol (Zhang et al.) entry into the brain is largely precluded by the BBB which is formed by endothelial cells. In the brain, cholesterol is synthesized de novo by all cells. Cholesterol synthesis starts from acetyl-CoA, the rate-limiting step is catalyzed by HMG-CoA reductase (HMGCR gene). SREBP (sterol regulatory element-binding protein) transcription factors induce cholesterol synthesis. Apolipoprotein $E$ (ApoE) containing lipoproteins that are generated by $A B C$ transporter (ABCA1) mediated secretion and lipidation, facilitate the horizontal transport of cholesterol. Lipoproteins are endocytosed by receptors of the lowdensity lipoprotein receptor (LDL-R) family. During myelination, cholesterol is integrated into the myelin sheath. One excretion route for cholesterol out of the brain is achieved by conversion to $24(\mathrm{~S})$-hydroxycholesterol (24-OHC) catalyzed by cholesterol 24-hydroxylasldle (CYP46). 24-OHC can pass the BBB into the blood stream where it gets transported for degradation. About one third of cholesterol is excreted by another route, potentially involving ApoE. Dashed arrows point to yet unknown pathways (modified from Saher and Stumpf, 2015). 


\subsection{Blood-brain barrier}

The Blood-brain barrier (BBB) has been experimentally observed by Paul Ehrlich in the late 19th century, following intravenous injection of a water-soluble dye that stained all peripheral organs except the structures of the CNS (Ehrlich, 1885). This phenomenon led to the depiction of a "capillary wall that must block the entrance of certain molecules" (Lewandowsky, 1900) followed by the designation as blood brain barrier (Barrière hématoencéphalique) by Stern and colleagues (Stern and Gautier, 1921). Later, specialized brain endothelial cells (BECs) forming the BBB were characterized by presence of unique belt-like tight junctions (Reese and Karnovsky, 1967), higher mitochondrial volume fraction (Oldendorf et al., 1977), low pinocytic and transcytosis activity (Coomber and Stewart, 1985; Peters et al., 1991), no fenestration (Fenstermacher et al., 1988), the presence of specialized transport systems (Abbott et al., 2010; Abbott et al., 2006), decreased expression of leukocyte adhesion molecules (Daneman et al., 2010), and a narrow paracellular cleft (interendothelial space) between adjacent endothelial membranes (Vorbrodt and Dobrogowska, 2003) (Figure 4).

\subsubsection{The Neurovascular Unit, Tight junctions and CNS transport}

BBB properties are orchestrated by cellular interaction of BECs with pericytes, perivascular glial cells, astrocytes and neurons, together establishing the Neurovascular Unit (NVU) (Alvarez et al., 2013; ladecola, 2017; Maoz et al., 2018; Muoio et al., 2014; Sa-Pereira et al., 2012). In the brain, microvessels are ensheathed by astrocyte end-feet, forming the glial limitans that covers almost the complete CNS surface. In CNS capillaries, BECs and astrocytes form two distinguishable basement membranes with pericytes and perivascular cells embedded into the endothelial basement membrane. The formed basement membrane, is characterized by classical extracellular matrix proteins like collagen, proteoglycans, laminin and fibronectin, secreted by cells of the NVU (Baeten and Akassoglou, 2011). The importance of cellular constituents of the NVU regulating BBB permeability may be highlighted by genetically induced pericyte deficiency, which results in increased transcytosis of tracer molecules and lipids into the CNS parenchyma by interfering with astrocyte end-feet polarization in the presence of intact tight junctions (Armulik et al., 2010; Saeed et al., 2014). 
A

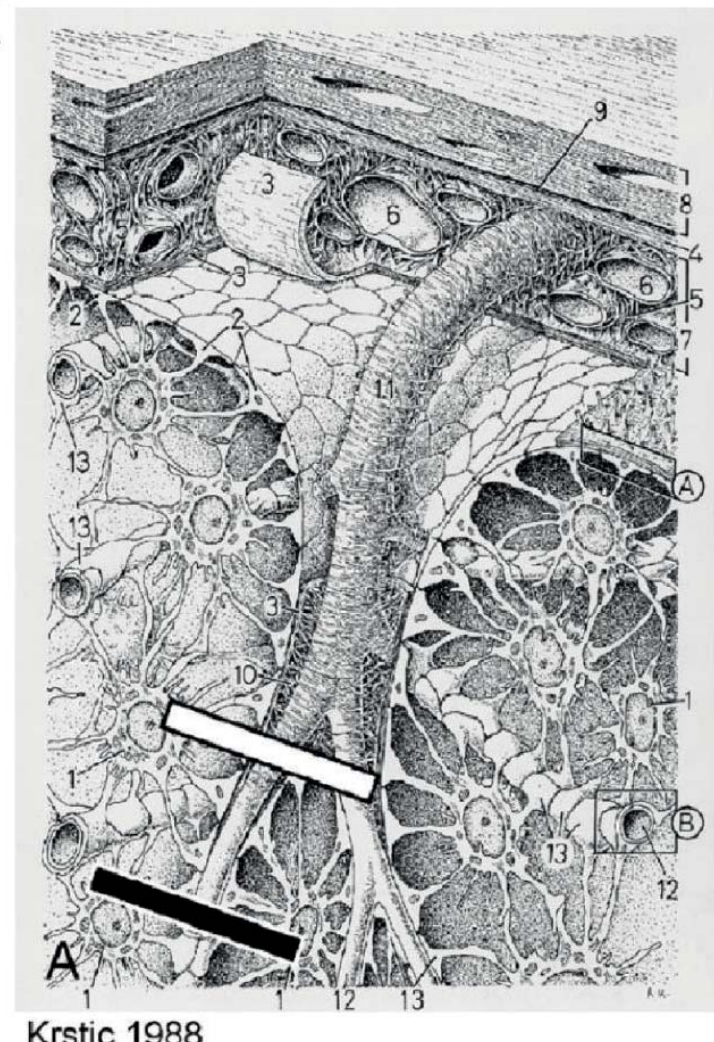

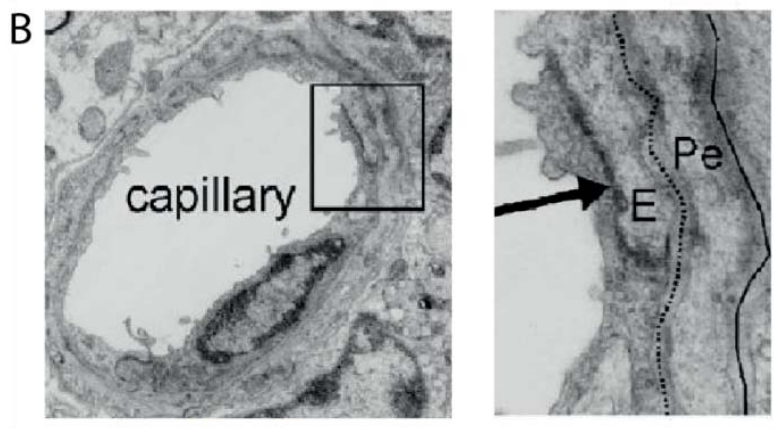

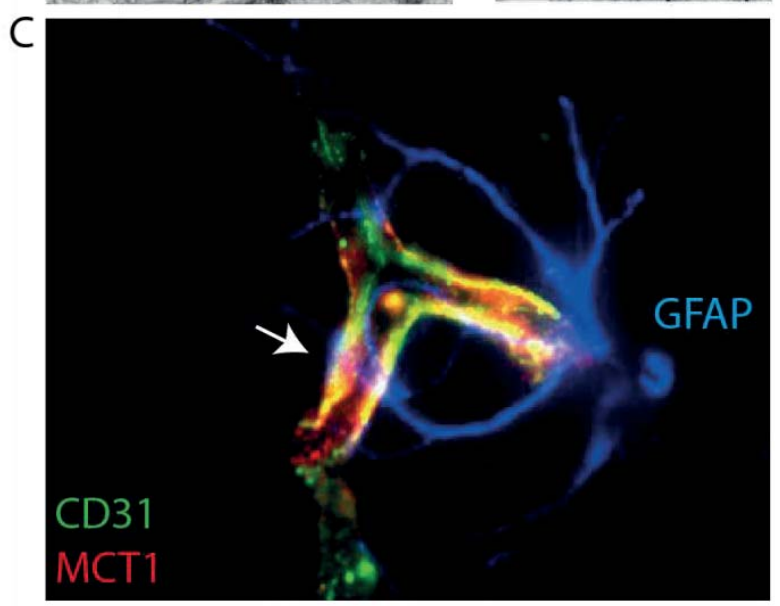

Figure 4: Anatomical structure of the neurovascular unit.

(A) Schematic diagram of different anatomic regions in the brain penetrated by vessels [11]. Superficial vessels of the brain [6] located in the subarachnoid space [7]. This compartment is delineated by the arachnoid mater [4] and the pia mater [3]. The surface of the brain is completely covered by the astrocytic endfeet of the glia limitans [2]. Toward the subarachnoid space, these endfeet are designated as glia limitans superficialis [A]. Inside the brain this coverage is termed glial limitans perivascularis $[\mathrm{B}]$. Perivascular space still connected to the subarachnoid space. 1 indicates perikaryon of an astrocyte; 2, glia limitans superficialis; 3, connective tissue of the pia mater (inner layer of the leptomeninges); 4 , arachnoid mater (outer layer of the leptomeninges); 5 , subarachnoid connective tissue (trabeculae arachnoideae); 6 , subarachnoid vessel; 7, subarachnoid space; 8, dura mater (pachymeninges); 9, neurothelium; 10, perivascular space; 11, penetrating vessel; 12, capillary; 13, glia limitans perivascularis. (B) "Corresponding to black bar". In capillaries (12) in (A), the basement membranes are merged to form a "fused gliovascular membrane" that occludes the perivascular space. (Upper right) Higher magnification of the field depicted (B). The capillary wall consists of endothelium $E$, endothelial basement membrane (dotted line), and pericytes Pe. The fused gliovascular membrane is shown by a continuous black line. It is directly opposed to the glia limitans. Black arrow point to overlap of adjacent endothelial cells with tight junctions in between (paracellular cleft). Figure reprinted from Radivoj V. Krstic: Die Gewebe des Menschen und der Säugetiere (Human and Mammalian Tissues), 1988, (adapted from Owens et al., 2008). (C) Astrocyte (glial fibrillary acidic protein, GFAP) with end-feet polarization at brain microvessel (CD31, PECAM1) colocalizing with monocarboxylat-transporter 1 (MCT1), enabling CNS influx of polar nutrients like pyruvate. 
In contrast, loss of astrocyte derived laminin impacts pericyte maturation leading to BBB breakdown accompanied by reduced expression of tight junction proteins in BECs (Yao et al., 2014). Tight junction proteins between adjacent endothelial cells are critically involved in maintaining BBB functioning by restricting paracellular passage of ions and small molecules (Abbott et al., 2010; Bazzoni and Dejana, 2004; Vorbrodt and Dobrogowska, 2003). Within CNS vasculature, family members of the claudin proteins (Claudin-1, $-2,-5$ and -12 ) with claudin-5 being the most abundant one (Greene et al., 2019; Morita et al., 1999), occludin (Morcos et al., 2001) and junctional adhesion molecules (JAMs) interact with scaffold proteins like zonula occludens-1 (ZO-1) (Jia et al., 2013). Together with adherens junction proteins, like vascular endothelial cadherin (VE-cadherin) or platelet and endothelial cell adhesion molecule 1 (PECAM-1) (Li et al., 2018; Stamatovic et al., 2008), these molecules determine BBB tightness, evaluated by loss of function in vivo and in vitro (Nitta et al., 2003; Umeda et al., 2006; Van Itallie et al., 2010; Wimmer et al., 2019). Several signaling molecules with barrier promoting properties have been identified. For instance, astrocyte dependent Wnt/ß-catenin and sonic hedgehog (Shh) signaling is directly involved in regulating the expression of tight junction proteins in BECs (Alvarez et al., 2011b; Daneman et al., 2009; Zhou et al., 2014). In addition, astrocyte derived factors like angiopoietin-1 (ANG1) (Saharinen et al., 2017), vascular endothelial growth factor (VEGF) (Rosenstein et al., 2010), transforming growth factor beta (TGF- $\beta$ ) (Dobolyi et al., 2012), glial-derived neurotrophic factor (GDNF) (Igarashi et al., 1999), and fibroblast growth factors (FGFs) (Reuss et al., 2003) have been linked to BBB maintenance. Recent attention has also focused on the erythropoietin producing hepatocellular carcinoma receptors-ephrin (EphR/Ephrin) system linked to BBB pathology in neurovascular and neuropsychiatric disorders (Malik and Di Benedetto, 2018). Together, these factors also enable regulated transport into the brain and allow only small lipophilic molecules and gases to freely enter the CNS (Abbott, 2013; Nag and Begley, 2005; Omidi and Barar, 2012). Metabolic supply of the brain that is connected to neuronal activity via neurovascular coupling involving astrocytes (Figley and Stroman, 2011; Petzold and Murthy, 2011), is essential for normal CNS functioning. Several transporters mediate shuttling of polar nutrients like glucose and amino acids at the BBB (Figure 4). Expression of transports of the solute carrier (SLC) superfamily by BEC, such as GLUT1 (SIc2a1) 
shuttling glucose, or monocarboxylate transporters like MCT1 (Slc16A1) transporting lactate, ketone bodies and pyruvate are critically involved maintaining CNS nutrient demands (Bergersen, 2015; Chiry et al., 2006; Halestrap, 2013; Tang et al., 2017). In addition, energy dependent luminal export via ABC-family transports such as Pglycoprotein (MDR1, Multidrug Resistance Protein 1) enables efficient removal of xenobiotics and natural lipophilic products that could be harmful for the brain (Loscher and Potschka, 2005). Transport of intact macromolecules, avoiding the lysosomal compartment, is coordinated by receptor-mediated or adsorptive-mediated transcytosis (Abbott et al., 2010; Nag and Begley, 2005) (Figure 5). These internalization routes involve several membrane domains including lipid rafts, caveolae and clathrin-coated pits resulting in a variety of traffic pathways like caveolae or clathrin-mediated endocytosis, fluid phase endocytosis and micropinocytosis to allow entry of macromolecules like insulin, transferrin or albumin (Omidi and Barar, 2012). BECs are characterized by an unusually low rate of transcytosis (see above). In a recent work, it has been suggested that endothelial cell lipid composition determined by the luminal lipid transporter major facilitator superfamily domain containing $2 a$ (Mfsd2a), specifically inhibits the caveolaemediated internalization pathway, highlighting regulated BBB permeability by controlled transcytosis (Andreone et al., 2017).

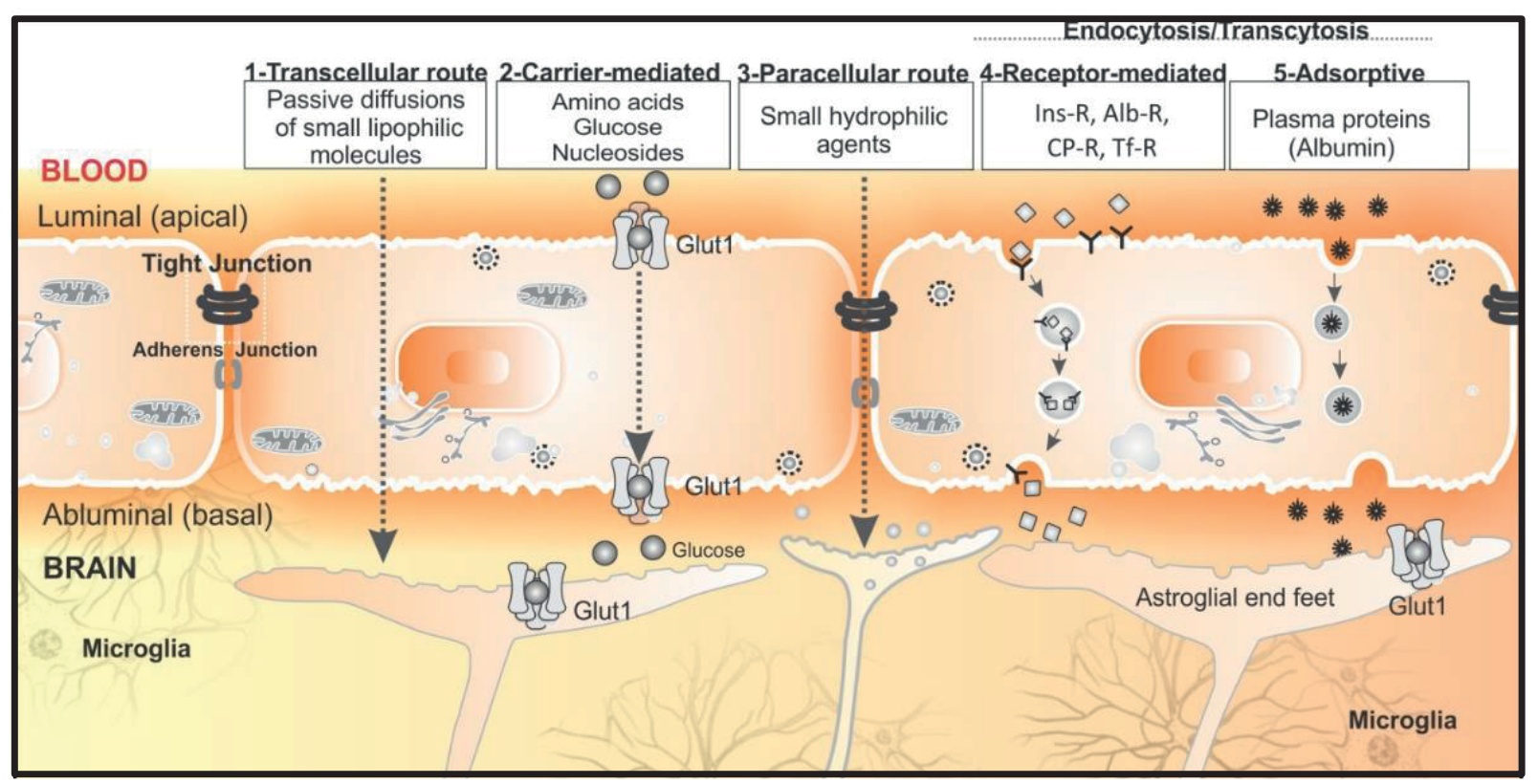


Figure 5: Transport pathways at the blood-brain barrier.

(1) Lipophillic small substrates (<500 Da) are able to diffuse across the membrane. (Marchiando et al.) Carrier-mediated transport machineries (e.g. Glut1, MCT1). (3) Paracellular pathway of some small hydrophilic agents. (4) and (5) Endocytosis/Transcytosis mediated transport routes of either large molecules via insulin receptor (Ins-R), albumin receptor (Alb-R), ceruloplasmin receptor (CP-R); transferrin receptor (Tf-R) or adsorptive-mediated endocytosis/transcytosis of e.g. albumin (adapted from Omidi and Barar, 2012).

\subsubsection{Blood-brain barrier during neuroinflammation}

Blood brain barrier breakdown and altered vascular permeability have been identified in several disorders affecting the CNS. In neurodegenerative diseases, including Multiple sclerosis (MS), pathological alteration of tight junctions and increased vascular permeability have been demonstrated (Rosenberg, 2012). However, BBB dysfunction in CNS diseases not only differs by extent but also by being a primary cause or evolving secondary to the primary insult. In MS, increased permeability of the BBB is an early occurring phenomenon during formation of new white matter lesion and can even be detected in inactive lesions and normal appearing white matter (Cramer et al., 2014). By studying MS tissue and experimental models, vascular hyperpermeability in MS has been shown to occur via several mechanisms: (1) Glial activation and secretion of proinflammatory factors, (Marchiando et al.) BBBEC activation and downregulation or disorganization of tight junction proteins and (3) perivascular infiltration and secretion of inflammatory products. Interestingly, increased vascular permeability appears to occur even before onset of symptoms as shown by MRI studies in patients and using the experimental autoimmune encephalomyelitis (EAE) mouse model (Davalos et al., 2014; Kermode et al., 1990; Kirk et al., 2003; Kirk and Karlik, 2003; Schellenberg et al., 2007). Further, increased glial fibrillary acidic protein (GFAP) expression before onset of EAE symptoms suggests early astrocyte activation and may indicate their importance in the context of vascular hyperpermeability (Luo et al., 2008). Astrocytes have been identified as important mediators of BBB integrity (see above) via release of growth factors, morphogens, cytokines and enzymes, regulation of blood flow in response to neuronal activity and control of water homeostasis by expressing the water-selective channel aquaporin 4 (AQP4) (Abbott et al., 2010; Abbott et al., 2006; Alvarez et al., 2011a; Luissint et al., 2012). In case of a neuroinflammatory event, astrocytes display 
a phenotype marked by hypertrophy and increased expression of GFAP, termed as reactive astrogliosis. Due to the close interaction of astrocytes and brain endothelia cells, reactive gliosis is accompanied by dysregulation of several BBB homeostasis mechanisms. For instance, increased vascular permeability during brain inflammation seems to be associated with an increase in intestinal fluid (edema) (Nag et al., 2009). In MS and corresponding animal models (EAE, Lysolecithin) increased astrocytic AQP4 expression is thought to counterbalance edema formation and BBB hyperpermeability. However, studies in AQP4 deficient animals have revealed a proinflammatory role of AQP4 expression in astrocytes (Li et al., 2011). Wosik and colleagues identified another mechanism of BBB dysregulation caused by disruption of angiotensin signaling in astrocytes. Therefore, astrocytic angiotensinogen expression seems to be crucial in maintaining occludin stability, that is downregulated in MS tissue most probably by inflammatory cytokines (Wosik et al., 2007). Several astrocyte derived inflammatory mediators and inducers of increased vascular permeability have been identified in experimental models and MS samples. Some of these, like vascular endothelial growth factor (VEGF) (Argaw et al., 2012; Argaw et al., 2009), tumor necrosis factor alpha (TNFA) (Marchiando et al., 2010), Interleukin$1 \beta$ (IL-1ß) (Ferrari et al., 2004; Wang et al., 2014), Interleukine-6 (IL-6) (Paul et al., 2003) and C-C motif chemokine ligand 2 (CCL2) (Stamatovic et al., 2003) have been shown to directly affect endothelial cell permeability in vivo and in vitro (Camire et al., 2015; Rochfort et al., 2014). Interestingly, astrocyte derived CCL2 has been shown to disrupt tight junction expression even before disease expression in EAE (Paul et al., 2014). In addition the inflammatory milieu is capable of inducing the expression of iNOS in reactive glia cells and eNOS in endothelial cells thereby downregulating tight junction proteins (Argaw et al., 2012; Fabis et al., 2007; Wang et al., 2016a). Together, these observations show that the inflammatory milieu, likely orchestrated by several brain cells, seems to closely correlate with altered vascular permeability and BBB breakdown. In MS, several mentioned inflammatory mediators affecting BBB integrity have been also attributed to activated microglia or invading macrophages (da Fonseca et al., 2014; Mahad and Ransohoff, 2003). BBB disruption mediated by activated microglia seems to depend on the interaction with other glial cells e.g. astrocytes via different cytokines/chemokines in an in vitro BBB model (Shigemoto-Mogami et al., 2018). Of note, besides disease specific pathways 
affecting BBB function, recent data indicate a core blood-brain barrier dysfunction module that is shared between different neurological disease entities, shifting BECs to a peripheral endothelial cell-like state (Munji et al., 2019).

\subsection{Multiple Sclerosis}

Multiple sclerosis (MS) is a chronic inflammatory, demyelinating and neurodegenerative autoimmune disease of the CNS, most commonly affecting young adults (Dobson and Giovannoni, 2019; Filippi et al., 2018; Goldenberg, 2012; Thompson et al., 2018). MS is the most common variant of broad spectrum idiopathic inflammatory demyelinating disorders (IIDDs) that range pathologically from highly localized forms to multifocal or diffuse variants. Although overlap with pathological characteristics of other IIDDs exist, MS has to be clinically diagnosed and separated from reminiscent acute disseminated encephalomyelitis (ADEM), acute MS (Marburg variant), Balo's concentric sclerosis (BCS) and Schilder's disease as well as monosymptomatic IIDDs e.g. optic neuritis or IIDDs with a restricted topographical distribution, such as neuromyelitis optica (NMO) (Canellas et al., 2007). Worldwide, over 2 million people are affected by MS with increasing prevalence since 1990 (Collaborators, 2019) and a higher incidence rate in females in contrast to males (2.3-3.5:1) that has also risen during the last decades (Harbo et al., 2013). Although the underlying diseases mechanisms still remain incomplete, causative environmental, genetic, and epigenetic factors likely interacting with modifiable risk factors have been identified. Of those environmental risk factors, low serum levels of vitamin D, smoking, early life obesity and infection with the Epstein-Barr virus are connected to disease development (Dobson and Giovannoni, 2019; Thompson et al., 2018). Genetic evidence for diseases cause was supported by familial forms of MS (increased heritability within families) and correlation of decrease MS risk with degree of relatedness (Harirchian et al., 2018; Thompson et al., 2018). Of the >200 risk genes identified (Parnell and Booth, 2017), MS genetic susceptibility and disease severity are associated with variants of the human leukocyte antigen (HLA) encoding the major histocompatibility complex (MHC) (Moutsianas et al., 2015), in addition to immune cell phenotypes induced by genetic variants of e.g. interleukin receptors (IL2RA and IL7RA), Lymphocyte function-associated antigen 3 (LFA-3), TNF receptor superfamily member 1A (TNFRSF1A), Nuclear Factor Kappa B Subunit 1 (NFKB1) 
and Tyrosine kinase 2 (TYK2) (International Multiple Sclerosis Genetics et al., 2007; Sawcer et al., 2014). Furthermore, genetic variants affecting cholesterol metabolism such as variants of nuclear receptor subfamily 1 Group $\mathrm{H}$ Member 3 (NR1H3 or LXRa) (Wang et al., 2016c), apolipoprotein E (APOE) (Pinholt et al., 2006), variants on chr8p23 near farnesyl-diphosphate farnesyltransferase 1 (FDFT1) (Smets et al., 2018) and cytochrome P450 family 27 subfamily B member 1 (CYP27B1) (Ramagopalan et al., 2011; Ross et al., 2014) are connected to MS disease. However, future studies have to implement environmental and genetic risk factors with focus on disease progression, disease phase and even subvariants of IIDDs, which may not only be relevant to identify diseases mechanisms but also for therapeutic management of MS.

\subsubsection{Multiple Sclerosis Pathology}

Clinically, MS patients are grouped into four major disease courses. Relapsingremitting MS (RR-MS) accounting for $85 \%$ of MS patients that is marked by phases of diseases exacerbation and improvement, secondary progressive MS (SP-MS) a diseases phase which 15-30\% of RR-MS patients will enter (Lorscheider et al., 2016), primary progressive MS (PP-MS) affecting around $10 \%$ of patients, characterized by continuous symptom worsening and progressive-relapsing MS (PRMS), a rare severe MS manifestation with gradual symptom worsening and diseases exacerbation without remission that $5 \%$ of patient develop (Goldenberg, 2012). First clinical presentation of a patient with an inflammatory demyelinating single attack is termed clinically isolated syndrome (CIS) that converts to MS in a range between $10 \%-85 \%$, depending on anatomical lesion location up to $41 \%-61 \%$ and has been suggested to be a result of a pre-clinical stage that triggers diseases establishment (Efendi, 2015; Miller et al., 2012). MS pathology is characterized by focal lesions that exhibit hallmarks like BBB breakdown, inflammatory cell infiltration (lymphocytes and macrophages), oligodendrocyte loss, demyelination, reactive gliosis, and axonal degeneration that allows separation from other CNS disorders. However, heterogeneity of MS pathology especially during early, usually perivascular lesion establishment has been reported. In biopsy studies, $50 \%$ of patients display pattern I lesions, characterized by immunoglobulin and complement deposits, whether $\mathrm{T}$ cell and phagocyte mediated demyelination (pattern II) and distal oligodendrogliopathy 
(pattern III) account for most of the remaining lesion characteristics in addition to rare cases of primary periplaque oligodendrocyte degeneration (Lucchinetti et al., 2000; Stadelmann et al., 2011). Histopathologic lesion pattern classification seems not to correlate with clinical presentation (Pittock et al., 2005) leading to the assumption of stage-dependent heterogeneity (Barnett and Prineas, 2004) or even the concept of a general homogenous mechanism that associates with defined MS (Breij et al., 2008). However, supporting a concept of distinct entities, imaging studies could show well defined lesion characteristics in pattern classified patients (Lucchinetti et al., 2008; Stadelmann et al., 2011), therapeutic intervention by plasmapheresis seem to depend on pattern classification (Keegan et al., 2005), pattern I and II MS results in a defined antibody serum profile to lipids and CNS derived peptides (Quintana et al., 2008) and within MS samples from cerebrospinal fluid (CSF), patients with pattern II or III MS differ significantly from patients with pattern I MS (Jarius et al., 2017). Interestingly, patient-dependent immunopathological heterogeneity, might be of relevance for personalized therapeutic intervention (Konig et al., 2008; Metz et al., 2014).

MS lesions are located in white matter tracts as well as in grey matter tissue. Cortical demyelination that is observable at all stages of disease, can be extensive and correlates with physical disability and cognitive impairment in MS (Calabrese et al., 2010). Nonetheless, detection of cortical lesions within living patients is limited ranging between $10-15 \%$ and although likely present during early stages of MS, they are most extensive in patients with progressive disease (Lassmann, 2018). In early disease, classical active focal white matter plaques are most numerous and decline in number by entering progressive phase of disease (Frischer et al., 2015). Acute active lesions are characterized by massive lymphocyte invasion and infiltration of myelin phagocytosing myeloid cells (Microglia/Macrophages) (Figure 6). Temporal staging of white matter lesions is possible by analysis of phagocyte activation profile and myelin components within macrophage lysosomes, allowing subgrouping of early active (acute), chronic active slowly expanding and inactive lesions (Frischer et al., 2015; Lassmann, 2013) (Figure 6). In addition, smoldering rim lesions can be identified by an inactive center surrounded by active microglia/macrophages (Frischer et al., 2015) (Figure 6). Lesion expansion of chronic active lesions is characterized by active phagocytosis and appearance of foamy phagocytes that have 
been suggested to promote MS disease progression (Hendrickx et al., 2017) (Figure 6). However, recent studies indicate anti-inflammatory, neurotrophic and repair promoting properties of foamy phagocytes (Grajchen et al., 2018).
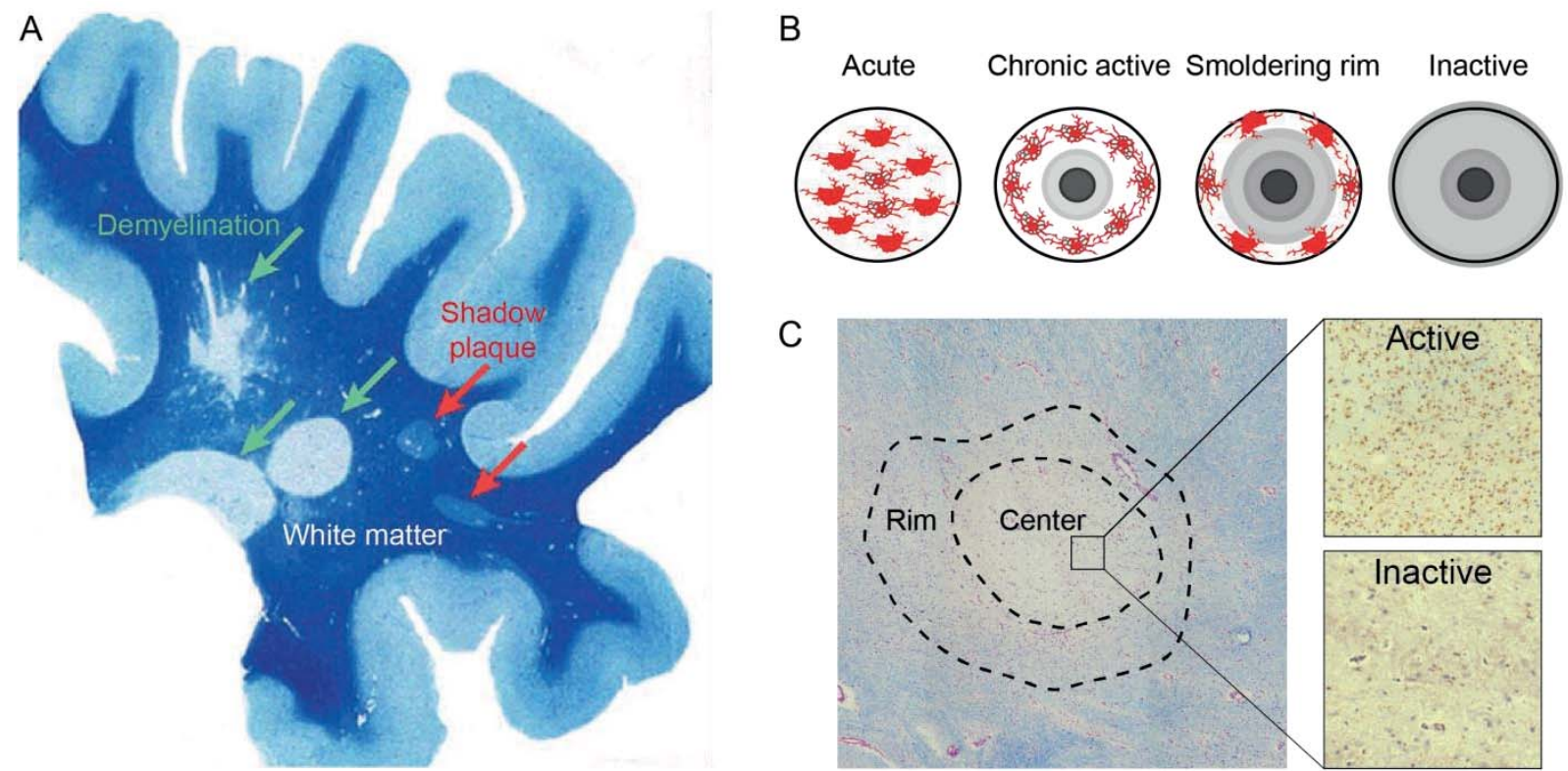

C
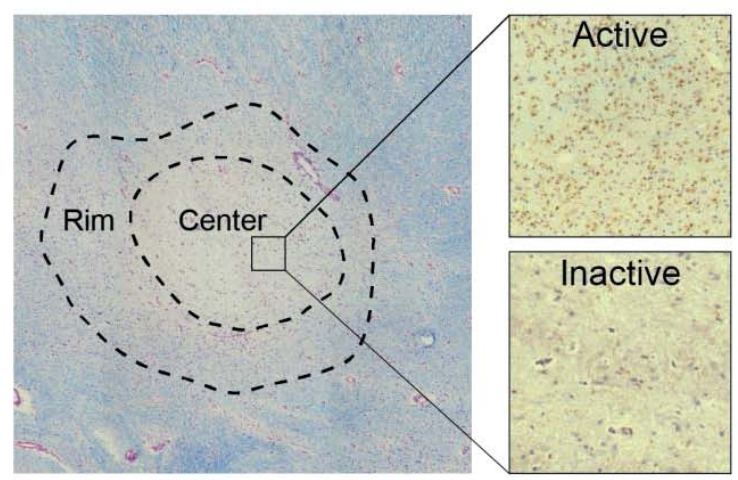

Figure 6: Pathology in Multiple Sclerosis

(A) Forebrain section of a MS patient stained with luxol fast blue (Albers et al.) to label myelin. Green arrows indicate demyelinated lesions. Red arrows indicate shadow plaques suggesting remyelination (adapted from Adams, 1989) (B) MS white matter lesion staging according to the topographical distribution of immunoreactive phagocytes and their myelin degradation products leading to foamy appearance. Acute active lesions are hypocellular with regularly distributed phagocytes containing myelin debris. Chronic active lesions with decreased phagocyte number in and around the inactive center with immunoreactive foamy phagocytes containing myelin degradation products clustered at the expanding lesion edges. Smoldering rim lesions are characterized by an inactive lesion center surrounded by microglia/macrophages rarely with myelin degradation products. Chronic inactive lesion are hypocellular but may still present inflammatory cells without early or late myelin degradation products (Grajchen et al., 2018; Popescu et al., 2013). (C) LFB stained white matter lesion with demyelinated center. Detection of active phagocytes within lesion center by CD68 labeling allows differentiation of lesion type/stage.

\subsubsection{Remyelination in Multiple Sclerosis}

In MS lesions, remyelination can be observed during all disease stages by the appearance of shadow plaques (Lassmann, 2013) (Figure 6). Remyelination can be extensive and has been suggested to rapidly follow demyelination but varies between patients (Patrikios et al., 2006; Prineas et al., 1989; Raine and Wu, 1993). It is assumed, that in $40 \%-50 \%$ of white matter lesions and up to $90 \%$ of grey matter lesions, remyelination occurs (Filippi et al., 2018). Newly generated myelin sheaths 
are thinner and remyelinated axons are marked by shortened internodal length despite the fact that remyelination enables axons to partially regain proper conduction properties (Duncan et al., 2017; Filippi et al., 2018; Scurfield and Latimer, 2018; Smith et al., 1979, 1981). Remyelination of lesions and functional reorganization of myelin components correlates with clinical remissions or recovery from relapses as well as with resolution of inflammation in MS (Mahad et al., 2008; Prineas et al., 1993a; Raine and Wu, 1993). Therefore, the number of myelin protein expressing oligodendrocytes correlate with degree of newly formed myelin (Lucchinetti et al., 1999). While in early MS, biopsy studies indicate extensive remyelination and an estimated proportion of failed remyelination of $20 \%$, this drastically decreases to only $15 \%$ remyelinated lesions in chronic MS with an increased proportion of $40 \%$ of failed remyelinated lesions (Goldschmidt et al., 2009; Nakahara, 2017). Thus, remyelination failure and decreased remyelination capacity that correlates with axonal loss, contributes to persistent disability in MS (Chari, 2007; Kornek et al., 2000). Myelin reestablishment has been suggested to rely on oligodendrocyte progenitor cell (OPC) repopulation, proliferation and differentiation in MS lesions (Franklin and Ffrench-Constant, 2008). In some MS patients, reduced numbers of OPCs have been reported, indicating either recruitment failure or depletion that gives rise to the concept of an exhausted pool of OPCs due to recurrent demyelination and age (Boyd et al., 2013; Brown et al., 2014; Franklin, 2002; Prineas et al., 1993b; Sim et al., 2002). However, a considerable number of OPCs within demyelinated lesions have been observed, pointing to a block of OPC differentiation (Chang et al., 2002; Franklin, 2002; Kuhlmann et al., 2008; Sim et al., 2002). Many extrinsic and intrinsic factors have been proposed that affect oligodendrocyte recruitment, proliferation and differentiation in MS lesions. Nonetheless, by analyzing lesion expression profiles, it was not possible to identify a clear oligodendrogliogenesis promoting or inhibiting expression pattern, arguing for temporal and mechanistic heterogeneity of MS lesions (Zeis et al., 2018). In addition, several studies using experimental model systems have underlined OPC heterogeneity with regards to their origin, location and response to demyelination in the adult brain (Crawford et al., 2016; Falcao et al., 2018; Marques et al., 2016; Vigano et al., 2013) that is further supported by identification of a different distribution of OPC subsets in MS patients (Jakel et al., 2019). Furthermore, it has been 
suggested that in addition to newly generated oligodendrocytes, adult surviving oligodendrocytes within lesions participate in remyelination (Duncan et al., 2018; Yeung et al., 2019) which is in contrast to the concept raised by experimental evidence, that post-mitotic, differentiated oligodendrocytes do not contribute to remyelination of demyelinated axons (Keirstead and Blakemore, 1997). These studies highlight the complexity of targeting remyelination in MS lesions to restore neurological disabilities. To date, limited remyelination in MS is still challenging and despite a wide range of anti-inflammation therapies, medication efficiently targeting remyelination is not available although over 88 different compounds in preclinical therapies, with 25 entering clinical trials, have been tested (Hooijmans et al., 2019).

\subsubsection{Animal models in Multiple Sclerosis research}

Several model systems to study MS have been introduced, however in general mimicking only aspect of the human disease (Bjelobaba et al., 2018; Kipp et al., 2012; Lassmann and Bradl, 2017; Procaccini et al., 2015; Ransohoff, 2012) this is supported by the limited success to transfer therapeutic candidates to human patients. Especially model systems that allow research with regards to inflammation, demyelination, axonal pathology and remyelination have been exploited, coming along with their advantages and disadvantages. Two major groups of rodent MS models may be separated by their pathologic entity: Toxin induced demyelination models and inflammatory demyelination models.

Several toxic compounds that generate demyelination have become an important tool to specifically study de-and re-myelination. Of those, cuprizone and lysolecithin induced lesions are extensively used in MS research. In mice, the oral administration of the copper chelator cuprizone (bis-cyclohexanone oxaldihydrazone) leads to a highly reproducible consistent demyelination of distinct brain regions, among them is the most investigated callosal white matter tract (corpus callosum; CC) (Carlton, 1967; Gudi et al., 2014; Kipp et al., 2009; Matsushima and Morell, 2001; Steelman et al., 2012; Torkildsen et al., 2008) (Figure 7). Although there is a lack of knowledge considering the exact molecular cause of cuprizone induced pathology, the cuprizone model exhibits some similarities to pattern III MS lesions (Gudi et al., 2014; Kipp et al., 2009; Lucchinetti et al., 2000; Praet et al., 2014). It has been assumed that 
copper chelation induces dysfunction of mitochondrial enzymes that selectively leads to caspase-3-dependend apoptosis of oxidative stress sensitive oligodendrocytes, already detectable after two to three days following cuprizone administration, mediated by activated glial cells (Clarner et al., 2015; Goldberg et al., 2013; Hesse et al., 2010). However, prominent myelin loss is only evident after 3 weeks of cuprizone intoxication that irreversibly initiates complete demyelination, accompanied by microgliosis, astrogliosis, axonal stress and OPC proliferation (Buschmann et al., 2012; Doan et al., 2013; Gudi et al., 2014; Gudi et al., 2009). Usually young adult C57BL/6 mice are fed $0.2 \%(\mathrm{w} / \mathrm{w})$ cuprizone to induce demyelination, avoiding increasing mortality rate from $<5 \%$ with $0.2 \%$ cuprizone to $10-15 \%$ with $0.3 \%$ cuprizone, with the drawback of higher variability between animals but less side effects like anorexia, ataxia, and tremors (Stidworthy et al., 2003; Torkildsen et al., 2008). Matsushima and Morell first characterized today's standard protocol over different administration periods and characterized the "acute demyelinationremyelination" time point following 6 weeks cuprizone exposure with observable complete remyelination after cuprizone removal from the diet, and the "chronic" demyelination phase for up to 16 weeks compound application, that can be interrupted by cuprizone removal inducing partial remyelination e.g. 12 weeks cuprizone followed by 2 weeks normal diet (Matsushima and Morell, 2001) (Figure 7). In addition to human pattern III MS lesion similarity, repeated cuprizone demyelination results in less efficient remyelination due to OPC depletion (Johnson and Ludwin, 1981; Mason et al., 2004), persistent disability (Irvine and Blakemore, 2006) and in aged animals, a decreased remyelination efficiency as well as axonal loss which is of relevance for chronic MS disease phase (Doucette et al., 2010; Manrique-Hoyos et al., 2012). Although cuprizone has been reported to lack major features like inflammatory infiltration of $\mathrm{T}$ cells and macrophages or blood brain barrier breakdown (Bakker and Ludwin, 1987; Boretius et al., 2012; Kondo et al., 1987; McMahon et al., 2002), several studies indicate that T cell subpopulation are involved in de- and re-myelination and vascular functioning might be affected (Dombrowski et al., 2017; Harsan et al., 2008; Kang et al., 2012; Thiessen et al., 2013). In addition, a novel modified cuprizone model in combination with EAE termed Cup/EAE model, leading to immune cell mediated demyelination has enormous potential for future studies (Ruther et al., 2017; Scheld et al., 2016). 
In contrast to cuprizone feeding, lysolecithin microinjection induced demyelination is applied in different selectable CNS tissues (Bjelobaba et al., 2018; Procaccini et al., 2015). Injection of the detergent-like lysophosphatidylcholine (lysolecithin) solubilizes myelin membranes, resulting in pronounced focal demyelination (Hall, 1972). Similar to cuprizone, lesion resolution and remyelination follows temporal predictability with remyelination occurring after phagocyte mediated removal of myelin debris (Bjelobaba et al., 2018). Although not extensive, following spinal cord induced lesions, Schwann cell remyelination has to be considered (Harrison, 1985). Like other toxic models, injection of lysolecithin does not induce an immune-mediated pathology although BBB is experimentally disrupted. Nonetheless, early extensive invasion of T and B cells is present (Bieber et al., 2003; Yazdi et al., 2015). In comparison to the cuprizone model, aged animals show reduced remyelination (Franklin et al., 2012; Shen et al., 2008), but repeated demyelination does not result in limited remyelination and reduced number of OPCs in the presence of constant degree of Schwann cell remyelination (Penderis et al., 2003).

One of the most established MS models accounting for the autoimmune component of MS is experimental autoimmune encephalomyelitis with all its different paradigms. There are several extensive reviews that critically evaluate EAE pathology available (Behan and Chaudhuri, 2014; Constantinescu et al., 2011; Glatigny and Bettelli, 2018; Gold et al., 2006; Robinson et al., 2014). In general, EAE is based on immunization of animals with myelin specific antigens, either by vaccination (active $E A E$ ), adoptive transfer with antigen specific $T$ cells (passive EAE) or myelin specific $T$ cell receptor transgenic mice that spontaneously develop EAE (Krishnamoorthy et al., 2006; Litzenburger et al., 1998; Pollinger et al., 2009; Waldner et al., 2004). Depending on the EAE model, acute and/or chronic, monophasic and/or relapsingremitting inflammatory demyelinating diseases is achieved.

Active immunization of susceptible animals can be performed by subcutaneous injection of antigen such as myelin basic protein (MBP), proteolipid protein 1 (PLP), myelin oligodendrocyte glycoprotein (MOG) or most widely used $\mathrm{MOG}_{35-55}$ peptide, emulsified in Complete Freund's Adjuvant (CFA) a mineral oil-based adjuvant 
containing heat-inactivated mycobacteria (Bjelobaba et al., 2018; Ransohoff, 2012). Immunization with CFA results in a strong delayed-type hypersensitivity immune response characterized by CD4+ T helper 1(Th1) lymphocytes (Billiau and Matthys, 2001; Laman et al., 1998). In contrast, immunization without bacterial components shifts immune response to Th2 profiles with strong antibody response (Billiau and Matthys, 2001). This has to be considered with regards to the classical concepts of Th1/Th2 dysregulation in MS (Jankovic and Feng, 2015; Laman et al., 1998; OrejaGuevara et al., 2012). To elicit pathology with clinical presentation, immune response is boosted by Bordetella pertussis toxin (PTX) injection post immunization, leading to T cell expansion, CNS immune cell entry and cytokine production (Richard et al., 2011; Waldner et al., 2004). Induction of EAE via CFA also affects BBB functioning likely contributing to the CNS pathology induction (Rabchevsky et al., 1999; Reiber et al., 1984). Following immunization, myelin specific $T$ cells are primed in secondary lymphoid organs leading to clonal expansion and differentiation to effector cells that egress lymphoid organs entering blood circulatory system through passing efferent lymphatic vessels (Rossi and Constantin, 2016). CNS entry is determined by adhesion molecules, cytokines/chemokines as well as their receptors and reactivation in the perivascular space by antigen-presenting cells, such as dendritic cells, microglia/macrophages and/or astrocytes (Engelhardt, 2006; Engelhardt and Coisne, 2011). Finally, infiltration of encephalitogenic effector $T$ cells and activation/recruitment of mononuclear cells induces demyelination and tissue damage, in classical C57BL/6 MOG-EAE is more pronounced in spinal cord tissue than in brain, accompanied by clinical presentation with ascending paralysis staring from the tail that can in severe cases, lead to complete hind and front limp paralysis (Bjelobaba et al., 2018; Kipp et al., 2012; Ransohoff, 2012) (Figure 7). Although clinical onset of disease coincides with lumbar infiltration of $T$ cells, mononuclear cells and white matter plaque formation that correlates with clinical disease severity, ascending paralysis is not solely attributed to axon sparing demyelination (Recks et al., 2011; Simmons et al., 1983). Therefore, several factors such as edema and perivascular inflammatory infiltrates likely contribute to disability. Nonetheless, clinical signs of EAE are dependent on the entry of inflammatory cells to CNS parenchyma passing both the BBB and the glial limitans (Engelhardt et al., 2016; Engelhardt and Coisne, 2011). During EAE, two phases are generally separated: An acute phase 
following diseases initiation with gradual clinical worsening reaching a peak after several days and a chronic phase with persistent disability or in case of monophasic/relapsing-remitting EAE a remission phase (Figure 7). During the chronic disease phase, inflammatory infiltrate differs from acute phase and has been linked to either differences in the inflammatory profile of a homogenous microglia/macrophage population or different phase specific contribution of microglia and macrophages to pathology (Yamasaki et al., 2014; Zorzella-Pezavento et al., 2013). In contrast to active EAE, adoptive transfer of encephalitogenic myelin specific effector $T$ cells results in a fast, monophasic and less severe disease, with minimal myelin destruction. This model has become an elegant research tool for principal mechanisms involved in the pathogenesis of $T$ cell mediated inflammation during effector phase of the disease providing insight into T cell traffic, activation and CNS infiltration (Engelhardt et al., 2016; Lodygin et al., 2019; Lodygin et al., 2013; Mundt et al., 2019). However, it has to be considered that EAE neither actively nor passively induced, provides insights into progressive phase of MS disease and lacks certain inflammatory players even leading to the assumption that EAE is a model for ADEM and not for MS (Constantinescu et al., 2011). In addition, remyelination cannot be studied well, due to simultaneous de- and remyelination events that are considered to be minor (Constantinescu et al., 2011; Ransohoff, 2012). 
Multiple Sclerosis

A

A Cuprizone

induced de- and remyelination

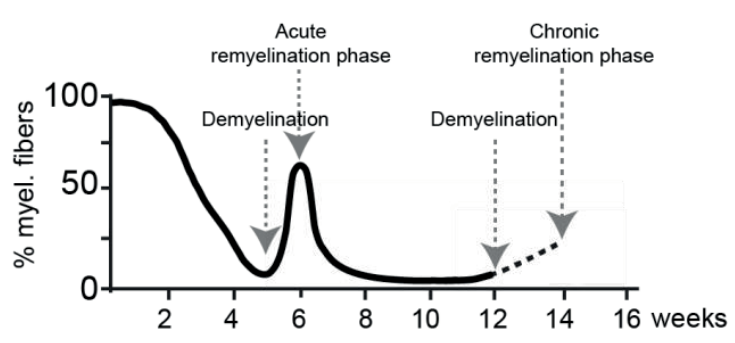

Acute remyelination

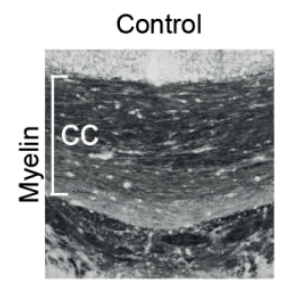

5 weeks

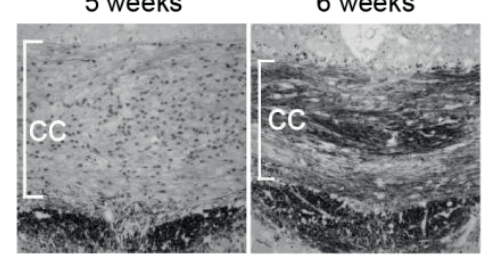

Chronic remyelination

12 weeks

+2 weeks

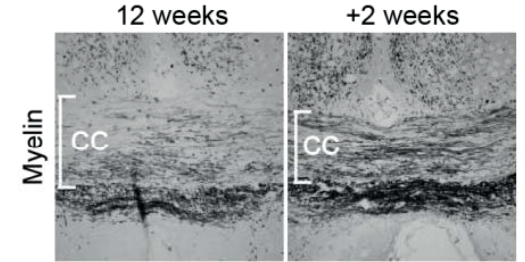

B

Experimental autoimmune encephalomyelitis (EAE)
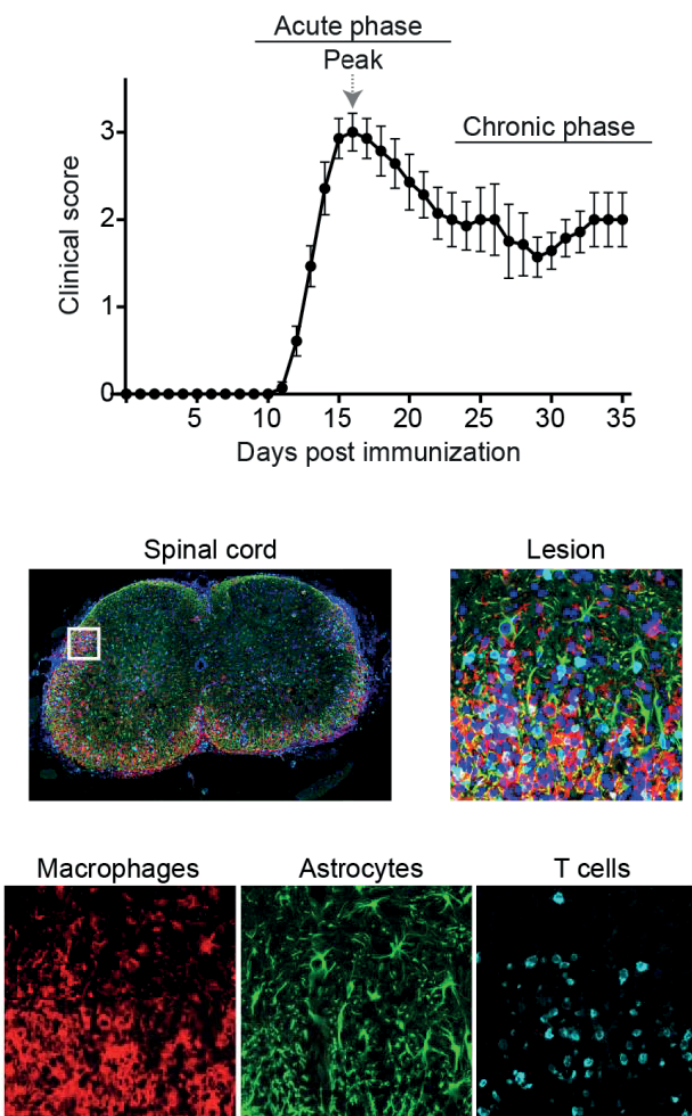

Astrocytes

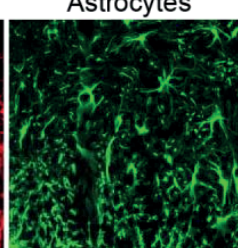

T cells

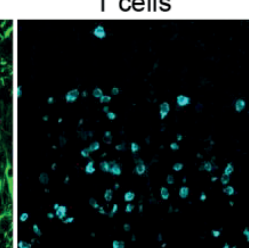

Figure 7: Mouse model in Multiple Sclerosis research

(A) Cuprizone de- and remyelination model. Scheme depicting the time course of demyelination and remyelination (upper panel) indicated by myelin labeling (Gallyas, lower panel) during cuprizone. During acute remyelination extensive number of remyelinated fibers can be observed, in contrast to decreased remyelination efficiency after chronic demyelination even after cuprizone removal (own data and Matsushima and Morell, 2001). (B) Clinical disease of chronic MOG $35-55$ EAE. (Upper panel) Animals usually start to show clinical symptoms (loss of tail tip tonus) 10 days following immunization. Maximum diseases expression (peak) with hind limp paralysis is reached within the following days with partial recovery leading to a persistent chronic disease phase. (Lower panel) Classical EAE lesions in the lumbar spinal cord at peak of disease visualized by co-labeling of microglia/macrophages (IBA-1), astrocytes (GFAP) and T cells (CD3). 


\section{Publications/Manuscripts}

\subsection{Publication 1:}

Dietary cholesterol promotes repair of demyelinated lesions in the adult brain

Stefan A. Berghoff ${ }^{1}$, Nina Gerndt ${ }^{1}$, Jan Winchenbach ${ }^{1}$, Sina K. Stumpf ${ }^{1}$, Leon Hosang $^{2}$, Francesca Odoardi ${ }^{2}$, Torben Ruhwedel ${ }^{1}$, Carolin Böhler ${ }^{1}$, Benoit Barrette ${ }^{1}$, Ruth Stassart ${ }^{1,3}$, David Liebetanz ${ }^{4}$, Payam Dibaj ${ }^{1}$, Wiebke Möbius ${ }^{1,5}$, Julia M. Edgar ${ }^{1,6}$ \& Gesine Saher ${ }^{1}$

${ }^{1}$ Department of Neurogenetics, Max Planck Institute of Experimental Medicine, Hermann-Rein-Str. 3, 37075 Göttingen, Germany.

${ }^{2}$ Institute of Neuroimmunology and Institute for Multiple Sclerosis Research, University Medical Centre Göttingen,Waldweg 33, 37073 Go"ttingen, Germany.

${ }^{3}$ Department of Neuropathology, University Medical Center, Georg-August-University, Robert Koch Str. 40, 37075 Göttingen, Germany.

${ }^{4}$ Department of Clinical Neurophysiology, Georg-August University, Robert Koch Str. 40, 37075 Göttingen, Germany.

${ }^{5}$ Center Nanoscale Microscopy and Molecular Physiology of the Brain (CNMPB), Wilhelmsplatz 1, 37073 Göttingen, Germany.

${ }^{6}$ Applied Neurobiology Group, Institute of Infection, Immunity and Inflammation, College of Medical Veterinary and Life Sciences, University of Glasgow, Glasgow G12-8TA, UK. 


\section{nature}

\section{COMMUNICATIONS}

ARTICLE

Received 22 Apr 2016 | Accepted 12 Dec 2016 | Published 24 Jan 2017

\section{Dietary cholesterol promotes repair of demyelinated lesions in the adult brain}

Stefan A. Berghoff', Nina Gerndt', Jan Winchenbach'1, Sina K. Stumpf ${ }^{1}$, Leon Hosang², Francesca Odoardi², Torben Ruhwedel', Carolin Böhler ${ }^{1}$, Benoit Barrette ${ }^{1}$, Ruth Stassart ${ }^{1,3}$, David Liebetanz $^{4}$, Payam Dibaj', Wiebke Möbius ${ }^{1,5}$, Julia M. Edgar ${ }^{1,6}$ \& Gesine Saher $^{1}$

Multiple Sclerosis (MS) is an inflammatory demyelinating disorder in which remyelination failure contributes to persistent disability. Cholesterol is rate-limiting for myelin biogenesis in the developing CNS; however, whether cholesterol insufficiency contributes to remyelination failure in MS, is unclear. Here, we show the relationship between cholesterol, myelination and neurological parameters in mouse models of demyelination and remyelination. In the cuprizone model, acute disease reduces serum cholesterol levels that can be restored by dietary cholesterol. Concomitant with blood-brain barrier impairment, supplemented cholesterol directly supports oligodendrocyte precursor proliferation and differentiation, and restores the balance of growth factors, creating a permissive environment for repair. This leads to attenuated axon damage, enhanced remyelination and improved motor learning. Remarkably, in experimental autoimmune encephalomyelitis, cholesterol supplementation does not exacerbate disease expression. These findings emphasize the safety of dietary cholesterol in inflammatory diseases and point to a previously unrecognized role of cholesterol in promoting repair after demyelinating episodes.

\footnotetext{
${ }^{1}$ Department of Neurogenetics, Max Planck Institute of Experimental Medicine, Hermann-Rein-Str. 3, 37075 Göttingen, Germany. ${ }^{2}$ Institute of Neuroimmunology and Institute for Multiple Sclerosis Research, University Medical Centre Göttingen, Waldweg 33, 37073 Göttingen, Germany. ${ }^{3}$ Department of Neuropathology, University Medical Center, Georg-August-University, Robert Koch Str. 40, 37075 Göttingen, Germany ${ }^{4}$ Department of Clinical Neurophysiology, Georg-August University, Robert Koch Str. 40, 37075 Göttingen, Germany. ${ }^{5}$ Center Nanoscale Microscopy and Molecular Physiology of the Neurophysiology, Georg-August University, Robert Koch Str. 40, 37075 Göttingen, Germany. ${ }^{5}$ Center Nanoscale Microscopy and Molecular Physiology of the
Brain (CNMPB), Wilhelmsplatz 1, 37073 Göttingen, Germany. ${ }^{6}$ Applied Neurobiology Group, Institute of Infection, Immunity and Inflammation, College of Medical Veterinary and Life Sciences, University of Glasgow, Glasgow G12-8TA, UK. Correspondence and requests for materials should be addressed to G.S (email: saher@em.mpg.de).
} 
n demyelinating diseases such as multiple sclerosis (MS), the failure to remyelinate contributes to axonal damage ${ }^{1}$, a major factor in persistent disability. Remyelination failure can be attributed partially to an insufficient capacity of resident oligodendrocyte precursor cells (OPC) to proliferate, migrate, differentiate and initiate myelin membrane growth ${ }^{2,3}$. There is now good evidence to implement therapies that combine the established immunosuppressive treatment of MS with compounds that stimulate remyelination and hence may secondarily limit axonal damage ${ }^{4,5}$. A number of factors that support differentiation of OPCs have been reported recently, some of which are linked to cholesterol metabolism in differentiating oligodendrocytes ${ }^{6-9}$.

Patients with MS have disturbed brain lipid metabolism ${ }^{10}$, but serum lipid profiles are in the normal range ${ }^{11}$. During active disease and disease progression, total cholesterol levels can rise to the upper limit of the normal range ${ }^{12-15}$. Increased dietary intake of cholesterol is assumed to increase serum cholesterol and stimulate immunological responses in inflammatory diseases ${ }^{16}$ However, it is unclear whether the elevated serum cholesterol in MS patients (i) contributes to disease progression, (ii) is a consequence of acute disease or (iii) reflects an attempt to counterbalance the pathophysiological manifestation of the disease.

We previously showed that cholesterol is rate limiting for CNS myelination ${ }^{17}$ and that nutritional cholesterol supplementation can stimulate developmental myelination in a mouse model of leukodystrophy ${ }^{18}$. Here, we investigate the effects of increased dietary cholesterol on disease parameters in three distinct mouse models of MS, that is, on (i) inflammation and demyelination in experimental autoimmune encephalomyelitis (EAE), (ii) remyelination in lysolecithin induced lesions and (iii) demyelination and remyelination in the cuprizone model. High-cholesterol chow does not aggravate clinical symptoms no inflammatory parameters in EAE or alter demyelination in cuprizone treated animals. Rather, we identify a novel function for cholesterol in myelin repair in adult mice. Dietary cholesterol modulates the profile of growth factors, simult aneously enhancing OPC proliferation and oligodendrocyte differentiation, thereby facilitating remyelination and reducing axonal injury. These data have implications for the treatment of demyelinating diseases.

\section{Results}

Cholesterol supplementation does not affect pathology in EAE To test whether elevated serum cholesterol is a biomarker of acute inflammatory disease, we induced MOG-EAE and determined serum cholesterol at the peak of clinical symptoms, typically 16-20 days after induction. Surprisingly, in acute EAE, tota serum cholesterol was reduced to about $75 \%$ of normal values $\left(76 \pm 2 \mathrm{mg} \mathrm{dl}^{-1} \pm\right.$ s.e.m. cholesterol in EAE mice compared with $103 \pm 2 \mathrm{mg} \mathrm{dl}^{-1}$ in untreated controls, $n=6-9, P<0.0001$ Student's $t$-test). Similar reductions were observed during remission at 28 days after immunization $\left(76 \pm 1 \mathrm{mg} \mathrm{dl}^{-1} \pm\right.$ s.e.m. $n=18, P<0.0001$ Student's $t$-test).

Next, we asked whether dietary cholesterol supplementation worsens acute inflammatory disease. Unexpectedly, mice on high-cholesterol chow $(5 \% \mathrm{w} / \mathrm{w}$ cholesterol, fat content unchanged) either prophylactically, two weeks before inducing MOG-EAE, or therapeutically with onset of clinical symptoms, showed similar disease onset (normal chow 12.6 \pm 0.3 days cholesterol $12.6 \pm 0.4 \mathrm{~d}, n=12-16$ ), mean clinical scores and body weight, as controls, during the 28 days of monitoring (Fig. 1a,b Supplementary Fig. 1). Moreover, high-cholesterol chow did not correct the reduced serum cholesterol $\left(77 \pm 8 \mathrm{mgdl}^{-1}, n=6\right)$. Correspondingly, at the peak of the clinical symptoms, dietary cholesterol did not influence the level of inflammation: histopathological lesions in the lumbar spinal cord white matter as well as the immune cell infiltration and characteristics of the pro-inflammatory milieu were comparable in extent and composition (Fig. 1c; Supplementary Fig. 2). These findings are in agreement with dietary cholesterol supplementation in the Theiler's virus model of MS (ref. 19). Nonetheless, inflammation was slightly ameliorated in cholesterol fed animals in remission, 28d after immunization (Fig. 1, Supplementary Fig. 2). Reduced infiltration of $\mathrm{T}$ cells and microglia/macrophages was accompanied by attenuated expression of several pro-inflammatory markers, such as interferon- $\gamma$ (IFN $\gamma$ ), interleukin 17 (IL-17), granulocyte-macrophage colony-stimulating factor (GM-CSF), tumour necrosis factor (TNF), and major histocompatibility complex II (MHCII). Taken together, EAE is associated with decreased serum cholesterol that is not restored by supplemented cholesterol. Importantly, cholesterol does not exacerbate disease but even slightly ameliorates inflammation during remission, suggesting it is safe to administer in inflammatory diseases. As cholesterol supplementation promotes developmental myelination ${ }^{18}$, these data prompted us to examine cholesterol supplementation in a remyelination paradigm.

Cuprizone lowers serum cholesterol and affects BBB integrity. We first tested whether serum cholesterol was altered in the cuprizone model of demyelinating disease (see also below). Surprisingly, after 4 weeks on cuprizone, mice had markedly reduced total serum cholesterol $\left(76 \pm 3 \mathrm{mg} \mathrm{dl}^{-1} \pm\right.$ s.e.m. in comparison to $103 \pm 3 \mathrm{mg} \mathrm{dl}^{-1}$ in controls, $n=9-13, P<0.0001$, Student's $t$-test). Although liver function values were normal (Supplementary Fig. 3), we cannot exclude the possibility that this is due in part to altered liver metabolism ${ }^{20}$. In contrast to EAE, dietary supplementation with $2 \% \mathrm{w} / \mathrm{w}$ cholesterol normalized total serum cholesterol $\left(106 \pm 5 \mathrm{mg} \mathrm{dl}^{-1}, n=13\right)$.

Under physiological conditions, the blood-brain barrier (BBB) prevents the passage of cholesterol from the circulation into the CNS (refs 21,22). Therefore, we tested whether dietary cholesterol could penetrate the CNS in cuprizone fed mice. Surprisingly, BBB integrity was compromised in mice treated with cuprizone for 4 weeks, as indicated by extravasation of Evans blue dye into the CNS, following systemic administration (Fig. 2a,b). Systematic evaluation revealed increased $\mathrm{BBB}$ permeability during the entire treatment period of up to 12 weeks of cuprizone feeding ( $1.4 \pm 0.1$ fold, $n=4 \mathrm{P}<0.05$ Student's $t$-test).

The extent of extravasation was much smaller than in EAE, likely explaining why previous studies have missed this BBB defect ${ }^{23-26}$. Notably, dietary cholesterol did not influence BBB permeability. When tested one week after a single injection of bodipy-cholesterol, the fluorescence from this cholesterol derivative (its biophysical properties are very similar to unmodified cholesterol ${ }^{27}$ ) was readily detectable in the corpus callosum of cuprizone fed mice (in contrast to untreated controls) with a pattern typical for an intracellular localization, potentially in glial cells (Fig. 2c). Quantification of extravasated bodipy-cholesterol revealed a $\sim 3$-fold increase in comparison to control mice (Fig. 2d). Thus, in cuprizone fed mice, peripheral cholesterol can cross the BBB.

Cuprizone mediated demyelination is unaltered by cholesterol. Next we tested whether nutritional cholesterol altered histopathology during the demyelination phase of cuprizone treatment (Fig. 3a) ${ }^{25,28}$. In the corpus callosum, oligodendrocyte loss and demyelination evolved over the same time course in control and cholesterol supplemented mice (Fig. 3b), leading to almost complete depletion of mature oligodendrocytes after 

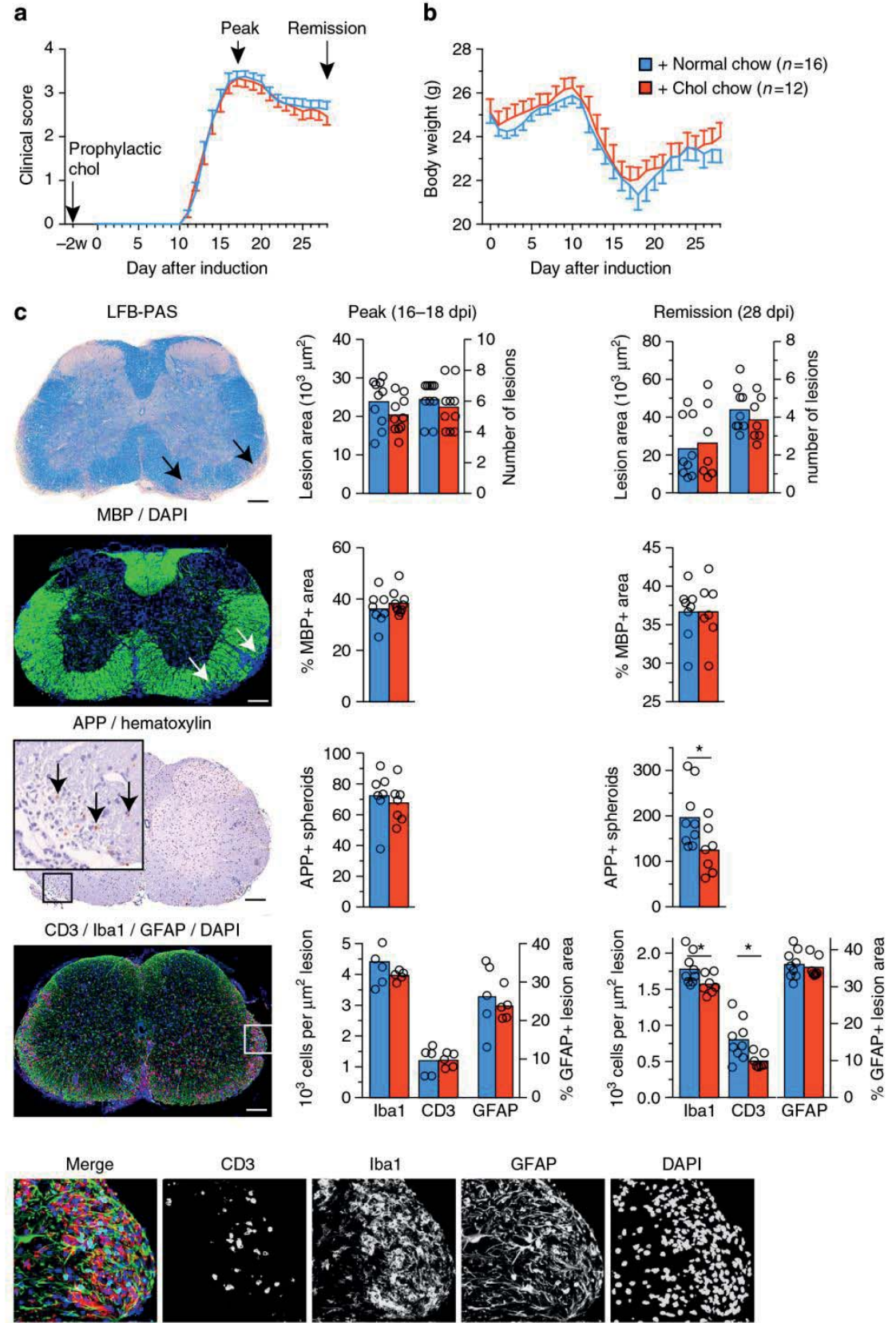

Figure 1 | Dietary cholesterol does not aggravate EAE pathology. (a) Clinical score of mice with MOG-EAE on normal chow or chow supplemented with $5 \%$ cholesterol ( $n=12-16$ mice, 2 independent experiments). Start of cholesterol feeding was prophylactic, two weeks before immunization. Arrows illustrate the time points of analyses at the peak of clinical symptoms (16-18dpi) and at remission (28 dpi). (b) Body weight of experimental animals as in (a) assessed from the day of induction of EAE to the end of monitoring clinical scores (28 days). Data is expressed as mean weight \pm s.e.m. of $n=12-16$ animals. Onset of clinical symptoms was paralleled by a drop in body weight, and mice gained weight only after the peak of disease. (c) Lesion characteristics were determined on sections of lumbar spinal cord from mice fed normal chow or cholesterol enriched chow ( $n=5$ animals, representative images on the left, scales $200 \mu \mathrm{m}$ ). Luxol fast blue-periodic acid-Schiff-hematoxylin (LFB/PAS) staining was used to determine the lesion area and number of lesions per section (arrow). Immuno-labeling for myelin basic protein (MBP) was used to determine the per cent of myelinated area within a lesion (defined in the DAPI channel as clusters of $>20$ nuclei, marked by arrows). On sections immuno-labeled for APP, the number of axonal speroids (arrows) per square $\mathrm{mm}$ white matter area was counted, as a readout of axonal damage. In remission, unpaired Student's $t$-test revealed significantly less axonal damage in cholesterol fed animals. Sections triple stained for microglia/macrophages, T cells, and astrocytes (Iba1-CD3-GFAP triple immuno-labeling) were used to assess the cellular composition of lesions. Unpaired $t$-tests revealed significantly reduced densities of microglia/macrophages and T cells in cholesterol fed animals $\left({ }^{\star}, P<0.05\right)$. Bars represent mean values with individual data points. 


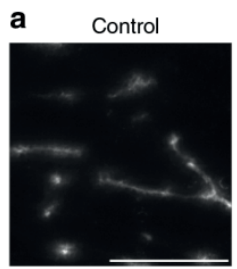

Evans blue

C

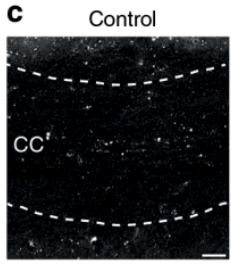

Bodipy-cholesterol

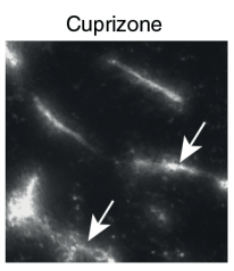

Cuprizone

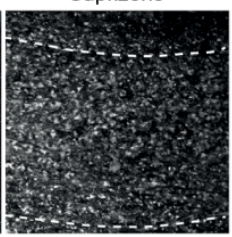

b

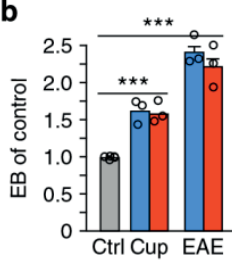

$\square$ Ctrl, control $\square+$ Normal chow $\square+$ Chol chow

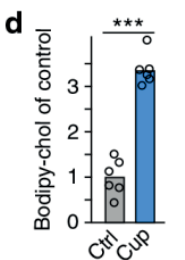

Figure 2 | Increased BBB permeability in cuprizone treated mice.

(a) Extravasation of Evans blue on sections of the corpus callosum. In control animals, Evans blue fluorescence is restricted to blood vessels but extravasates in mice on cuprizone (arrows) (scale, $50 \mu \mathrm{m}$ ). (b) BBB permeability was measured by Evans blue (EB) extravasation in brains of animals fed cuprizone (cup) for 5 weeks on normal chow or cholesterol supplemented chow, or in brains of animals with EAE $2 \mathrm{~d}$ after the peak of clinical symptoms ( $n=3$ animals). All treatment groups were normalized to untreated control animals $(n=5)$ and compared by one way ANOVA $(P<0.0001)$. Nutritional cholesterol did not influence BBB permeability. Bars represent mean \pm s.e.m. (c) Extravasation of bodipy-cholesterol. Maximum intensity projection of bodipy-cholesterol fluorescence in the corpus callosum (delineated by dashed lines) of mice that were kept on cuprizone for 5 weeks in comparison to untreated mice (control) (scale, $50 \mu \mathrm{m}$ ). (d) Quantification of bodipy-cholesterol extravasation after extraction. Data are expressed as fold changes \pm s.e.m. in cuprizone treated mice compared with untreated control animals $(n=6$ mice per group, unpaired Student's $t$-test, $P<0.0001)$.

four weeks. In addition, oligodendroglial numbers (Olig2, oligodendrocyte lineage transcription factor 2 marks OPCs and oligodendrocytes), astrogliosis (GFAP, glial fibrillary acidic protein) and microgliosis (MAC3, macrophage-3 antigen) steadily increased in a comparable manner in both groups, and axonal damage (APP positive spheroids, Fig. 3c) was similar at all time points tested. Taken together, cholesterol supplementation does not interfere with the cuprizone treatment, and mature oligodendrocytes do not escape the toxic insult.

Cholesterol facilitates remyelination and motor learning. Next, we tested the hypothesis that dietary cholesterol supplementation enhances adult remyelination. When mice are continuously exposed to cuprizone, an episode of spontaneous repair occurs in the sixth week, resulting in marked remyelination (Fig. $4 a)^{25,29}$. At this time point, cholesterol neither influenced oligodendrocyte numbers, remyelination nor glial responses (Fig. 4b,c). However, the density of APP positive axonal spheroids in cholesterol fed animals was reduced, suggesting attenuated axonal damage (Fig. 4d).

After chronic cuprizone exposure (12 weeks), a second episode of weak and transient remyelination (up to $20 \%$ of full myelination) occurs (Fig. 4a). However, even if cuprizone is withdrawn at this point, repair is very limited ${ }^{30}$. Thus, despite a considerable density of OPCs and mature oligodendrocytes, remyelination is marginal and astrogliosis substantial (Fig. 4b, blue bars at 12 weeks). Remarkably, cholesterol supplementation increased remyelination $\sim 1$.6-fold as assessed in Gallyas silver impregnated sections (Fig. 4b) and in electron micrographs of the corpus callosum (Fig. 4c, Supplementary Fig. 4). Coupled to this, a similar increase in OPCs and in mature oligodendrocytes was observed (Fig. 4b, 12 weeks). In addition, the positive influence of cholesterol was associated with increased body weight (Supplementary Fig. 5). Thus, in the context of recurrent depletion of mature oligodendrocytes, cholesterol supplementation enhances tissue repair.

To specifically determine the effect of cholesterol during remyelination, we exposed mice to cuprizone for four weeks to achieve complete demyelination, then withdrew cuprizone to induce remyelination ('induced remyelination') (Fig. 5a). Mice fed normal chow during the first 7 days after cuprizone withdrawal demyelinated further and had only slightly increased oligodendrocyte densities (Fig. 5b, compare blue bars 4 and $4+1$ ). In contrast, cholesterol supplementation following cuprizone withdrawal dramatically increased OPC proliferation and augmented Olig2 positive cell density 1.5 -fold (Fig. 5b,c). Densities of newly differentiated TCF4 + PCNA - (TCF4, also called TCF7L2, transcription factor 7-like 2; PCNA, proliferating cell nuclear antigen) oligodendrocytes were also increased by cholesterol (Fig. 5d, Supplementary Fig. 6), similarly as found in actively repairing lesions from patients with $\mathrm{MS}^{31-33}$. The resulting 2.7-fold increase in mature oligodendrocytes (Fig. 5b, time point $4+1$ ) led to a 1.8 -fold increase in myelin content on Gallyas silver impregnated sections and on electron micrographs (Fig. 5e; Supplementary Fig. 4). Cholesterol supplementation also altered the glial response, leading to a $\sim 30 \%$ increase in astrocytes and $\sim 50 \%$ reduction in microglial cells (Fig. 5b, $4+1)$. Axonal damage was attenuated to $\sim 70 \%$ in cholesterol fed animals of controls (Fig. 5f). These histological signs of repair were associated with a net gain in body weight, occurring within 7 days of cholesterol supplementation and contrasting with weight maintenance in mice fed normal chow (Supplementary Fig. 5). The beneficial effect of cholesterol persisted, leading to a robust increase in mature oligodendrocytes and myelin content at 2 weeks after cuprizone withdrawal (Fig. 5b, $4+2$ ); a result that was confirmed on electron micrographs (Fig. 5e).

To examine the generality of this response, we investigated whether dietary cholesterol enhanced remyelination in another, completely distinct in vivo model of remyelination that is accompanied by confined BBB disruption. Localized injection of lysolecithin into the ventral-lateral spinal cord of adult mice was used to produce focal demyelination. As in the cuprizone model, demyelination was associated with a reduction in serum cholesterol to about $70 \%$ of untreated controls. Further, dietary cholesterol ( $2 \% \mathrm{w} / \mathrm{w}$ for 14 days) increased serum cholesterol slightly $\left(79 \pm 3 \mathrm{mg} \mathrm{dl}^{-1} \pm\right.$ s.e.m. in cholesterol fed mice compared with $72 \pm 6 \mathrm{mg} \mathrm{dl}^{-1}$ in chow fed controls, $\left.n=3-5\right)$, enhanced remyelination and significantly increased the density of oligodendroglial cells within the lesion (Fig. 6a-c). The beneficial effect of cholesterol was also reflected in significantly increased body weight, relative to chow fed mice (Fig. 6d).

To investigate whether the histopathological improvements in cholesterol fed animals was associated with improved clinical measures, we returned to the 'induced remyelination' paradigm in the cuprizone model (for a scheme of experimental paradigm, see Supplementary Fig. 7), measuring the maximum running velocity (Vmax) on a running wheel. First, a training wheel with regularly spaced rungs was placed into the cages to improve cardiopulmonary and musculoskeletal strength. One week after cuprizone withdrawal, the training wheel was replaced by a complex wheel with irregularly spaced rungs to measure bilateral sensorimotor 
$$
\text { a }
$$
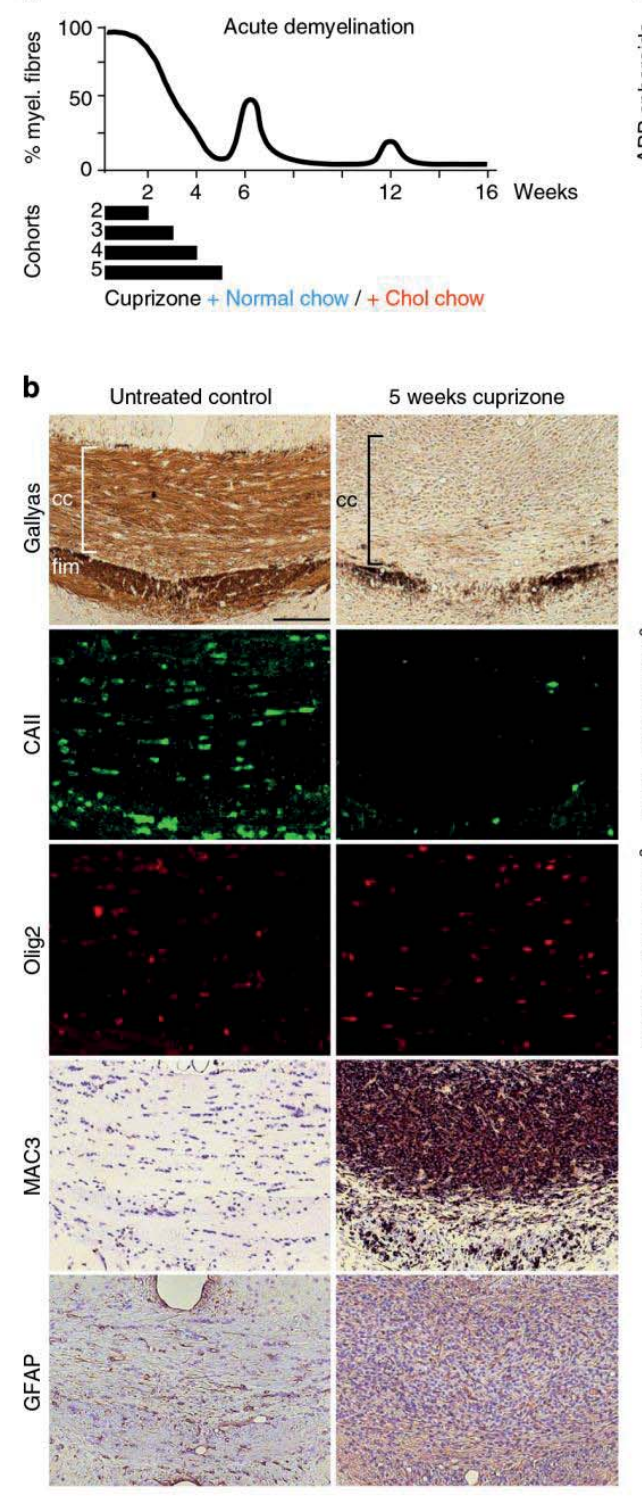

c

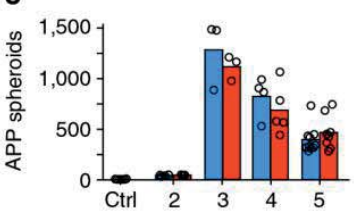

$\square$ Untreated contro

$\square+$ Normal chow

$\square+$ Chol chow
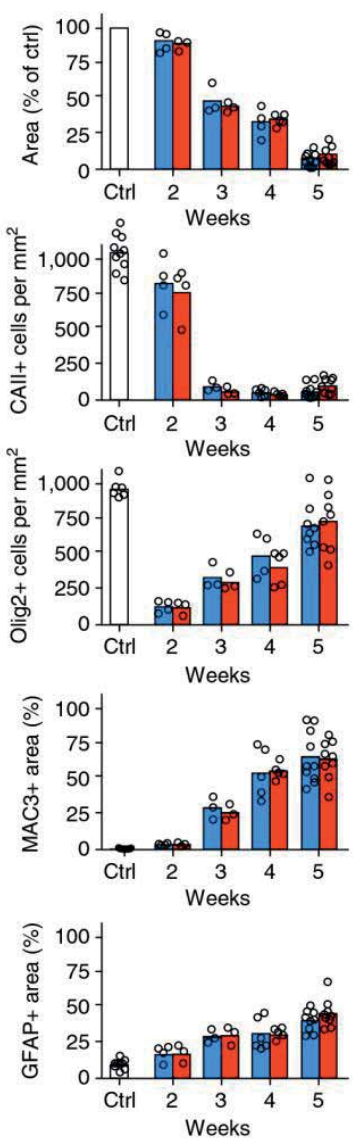

Figure 3 | Cholesterol does not affect cuprizone mediated demyelination. (a) Scheme depicting the time course of demyelination/remyelination during 6 week cuprizone feeding (upper panel, based on own results and on other studies ${ }^{25,28}$ ) to the treatment paradigm. To assess the influence of highcholesterol feeding on demyelination, mice on normal chow or high-cholesterol chow additionally received cuprizone in the diet for between 2 and 5 weeks (black bars) after which mice were analysed histologically. (b) Representative pictures of the corpus callosum of untreated control mice and mice after 5 weeks on cuprizone with the corresponding quantification on the right. Assessed were myelination (Gallyas silver impregnation), the number of mature oligodendrocytes (CAII), the number of oligodendrocyte lineage cells (Olig2), activated microglia (MAC3) and astrocytes (GFAP). Each bar represents the mean value for 3-5 (week 2-4) or 9-10 (week 5; untreated controls, ctrl) animals per condition with individual data points (scale $100 \mu \mathrm{m}$ ). (c) APP positive spheroids per $\mathrm{mm}^{2}$ in the corpus callosum at the end of 2-5 weeks of cuprizone with or without cholesterol supplementation ( $n=3-4$ animals at 2 and 3 weeks, $n=4-5$ at week $4, n=6$ untreated controls, $n=9-10$ at week 5).

coordination $^{34}$ (Supplementary Fig. 7). The Vmax of mice remyelinated on normal chow dropped to about $40 \%$ of levels on the training wheel, and did not improve above $75 \%$ (Fig. 5g). In contrast, mice receiving cholesterol supplementation showed a less severe drop in $\mathrm{V} \max$ (to 63\%), followed by a steady increase that reached the velocity achieved on the training wheel after two weeks. Importantly, in control mice (without cuprizone) cholesterol supplementation, did neither influence performance on the training wheel nor motor learning ( $\mathrm{Vmax}$, run duration, number of runs and running distance on the complex wheel) 


\section{a}

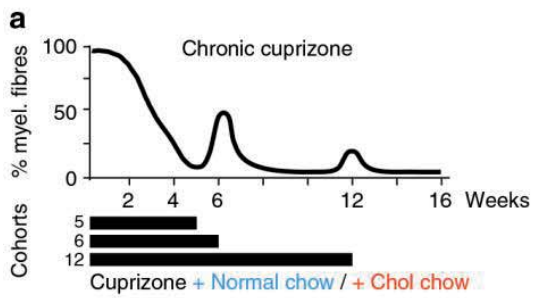

b

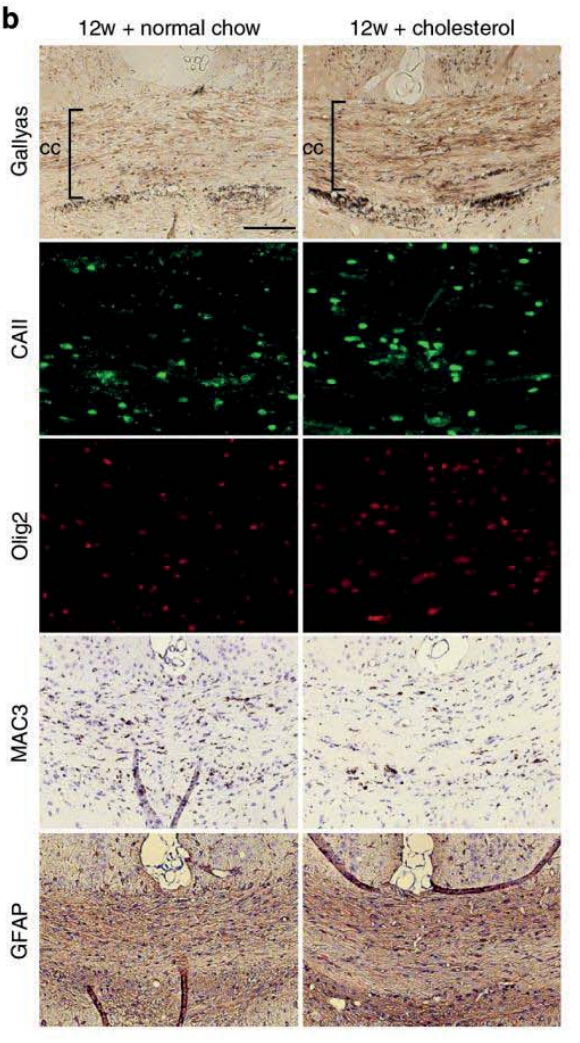

$\square$ Untreated control
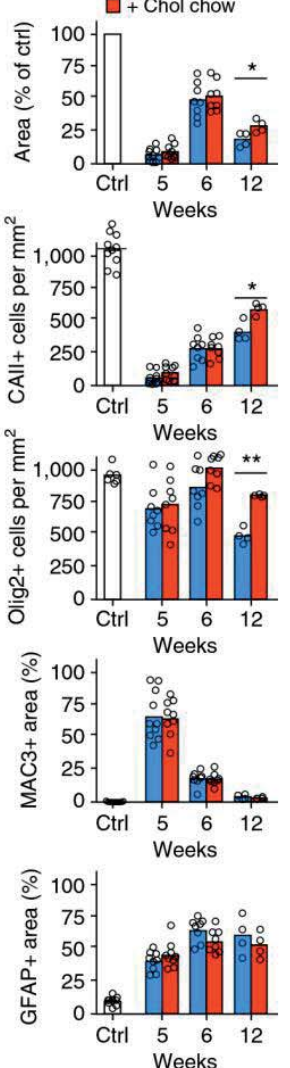

c

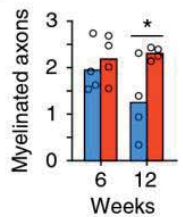

d

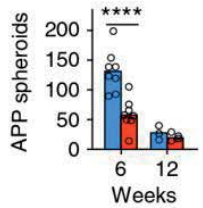

Figure 4 | Cholesterol facilitates remyelination after chronic cuprizone exposure. (a) Scheme depicting the time course of demyelination/remyelination during cuprizone feeding to the treatment paradigm. To assess the influence of high-cholesterol feeding on spontaneous remyelination, mice received cuprizone in normal chow or chow supplemented with cholesterol for 5,6 or 12 weeks (black bars) after which mice were analysed histologically.

(b) Evaluation of disease in the corpus callosum of mice that were treated with cuprizone for 5, 6 or 12 weeks on normal chow or chow enriched with cholesterol. Corresponding representative pictures of the 12 weeks treatment cohort are on the left. Assessed were myelination (Gallyas silver impregnation), the number of mature oligodendrocytes (CAll), the number of oligodendrocyte lineage cells (Olig2), activated microglia (MAC3) and astrocytes (GFAP). Each bar represents the mean value of 4 (week 12) or 8-10 (week 5,6) animals per condition with individual data points (scale $100 \mu \mathrm{m}$ ). (c) Myelinated axons per $10 \mu \mathrm{m}^{2}$ in the corpus callosum at the end of 6 and 12 weeks of cuprizone with or without cholesterol supplementation ( $n=4$ animals, Two-way ANOVA and Sidak's post test). (d) APP positive spheroids per $\mathrm{mm}^{2}$ in the corpus callosum at the end of 6 and 12 weeks of cuprizone with or without cholesterol supplementation ( $n=3-8$ animals, Two-way ANOVA and Sidak's post test). Asterisks represent significant differences with ${ }^{\star} P<0.05 ;{ }^{\star \star} P<0.01 ;{ }^{\star \star \star \star} P<0.0001$.

(Supplementary Fig. 7 and not shown). Hence, cholesterol supplementation enhances repair after demyelination and improves neurological outcomes by supporting oligodendrocyte proliferation and differentiation, promoting remyelination, decreasing microgliosis, and attenuating axonal damage in a permissive environment ('induced remyelination' after cuprizone withdrawal).
Cholesterol changes the expression profile of growth factors. To obtain insight into the mechanism by which cholesterol supports the simultaneous expansion of OPC and oligodendrocyte densities, we monitored differentiation of cultured primary oligodendrocytes in defined Sato media, with or without cholesterol supplementation. Oligodendrocytes differentiated significantly faster in the presence of cholesterol, as indicated by expression of differentiation markers and morphological changes 
a

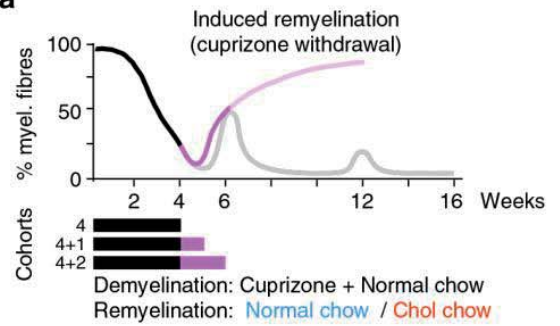

b

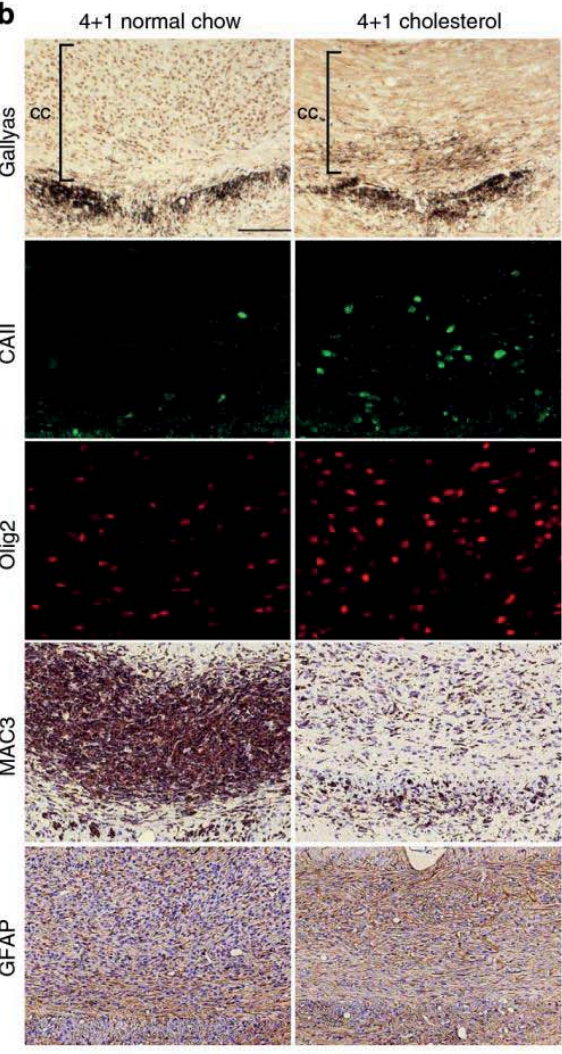

g
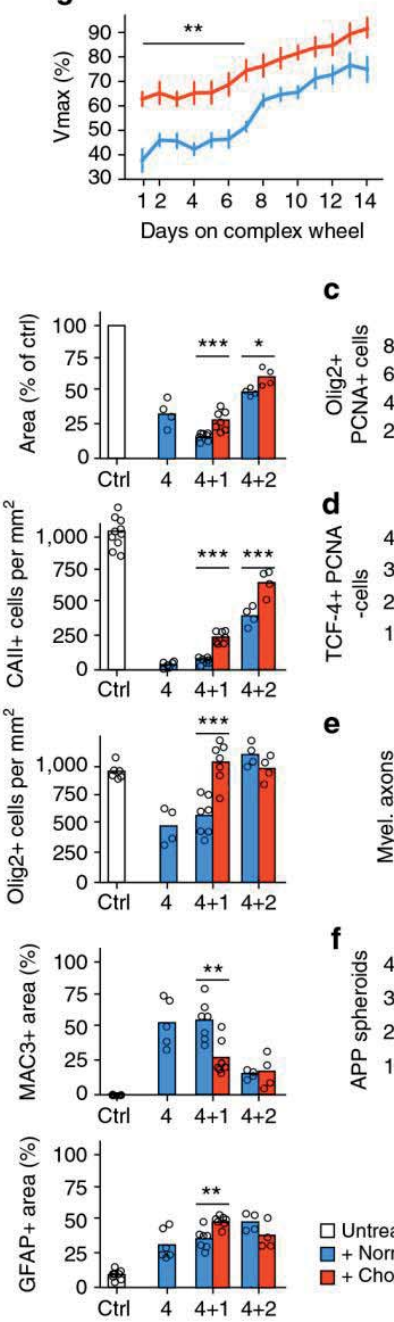

e

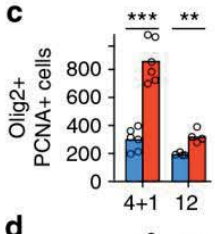

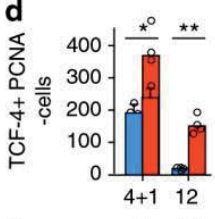
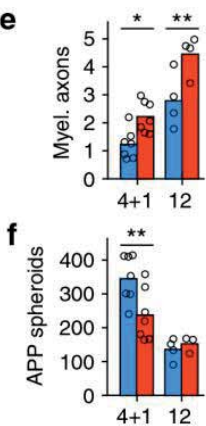

Untreated contro

$\square+$ Normal chow $\square+$ Chol chow

Figure 5 | Cholesterol facilitates remyelination after cuprizone withdrawal. (a) Scheme depicting the time course of demyelination/remyelination during cuprizone feeding (remyelination after cuprizone withdrawal in purple). The influence of cholesterol on remyelination was assessed by feeding mice cuprizone in normal chow for 4 weeks (4, black bars) followed by 'induced remyelination' after cuprizone withdrawal for $1(4+1)$ or $2(4+2)$ weeks on normal chow or cholesterol supplemented chow. (b) Representative pictures of the corpus callosum of mice after one week $(4+1)$ remyelination. Corresponding quantification is on the right also including values for 2 weeks remyelination $(4+2)$. Assessed were myelination (Gallyas silver impregnation), the number of mature oligodendrocytes (CAII), the number of oligodendrocyte lineage cells (Olig2), activated microglia (MAC3), and astrocytes (GFAP). Each bar represents the mean value from $n=4-5(4$ and $4+2)$ or $n=7(4+1)$ animals (scale, $100 \mu$ m; Two-way ANOVA and Sidak's post test). (c) Quantification of proliferating OPCS (PCNA positive Olig2 positive) in the corpus callosum of mice after $4+1$ treatment paradigm ( $4+1)$ or after 12 weeks (12) of cuprizone. Each bar represents the mean of $n=6-7$ (week $4+1$ ), or $n=4$ (week 12) animals (Student's $t$-test). (d) Quantification of newly differentiated postmitotic oligodendrocytes (TCF4 positive, PCNA negative) in the corpus callosum treated as in c). Each bar represents the mean of $n=6-7$ (week $4+1$ ), or $n=4$ (week 12) animals (Student's t-test). (e) Myelinated axons per $10 \mu \mathrm{m}^{2}$ in the corpus callosum at the end of the $4+1(n=7)$ and $4+2(n=4)$ treatment paradigm (two-way ANOVA and Sidak's post test). (f) APP positive spheroids per $\mathrm{mm}^{2}$ in the corpus callosum $(4+1 n=7 ; 4+2 n=3-4$ animals, two-way ANOVA and Sidak's post test). ( $($ ) Motor learning as assessed by maximum velocity (Vmax) on a complex wheel ( $n=6$ animals), expressed as per cent of the Vmax on a training wheel (mean of the last 7 days before changing to a complex wheel). Statistical evaluation of Vmax was done by Two-way ANOVA (cholesterol effect $P<0.0001$ ) and Sidak's post tests. Asterisks represent significant differences with ${ }^{\star} P<0.05 ;{ }^{\star \star} P<0.01 ;{ }^{\star \star \star} P<0.001$.

(Fig. 7a, Supplementary Fig. 8a). However, the final stage of maturation after $5 \mathrm{~d}$ in culture was unchanged, as shown previously $^{18}$. Similarly, the rate of myelination as measured by MBP (myelin basic protein) positive area per axonal area (SMI31, phosphorylated axonal neurofilaments), was increased in spinal cord co-cultures differentiated in the presence of cholesterol 

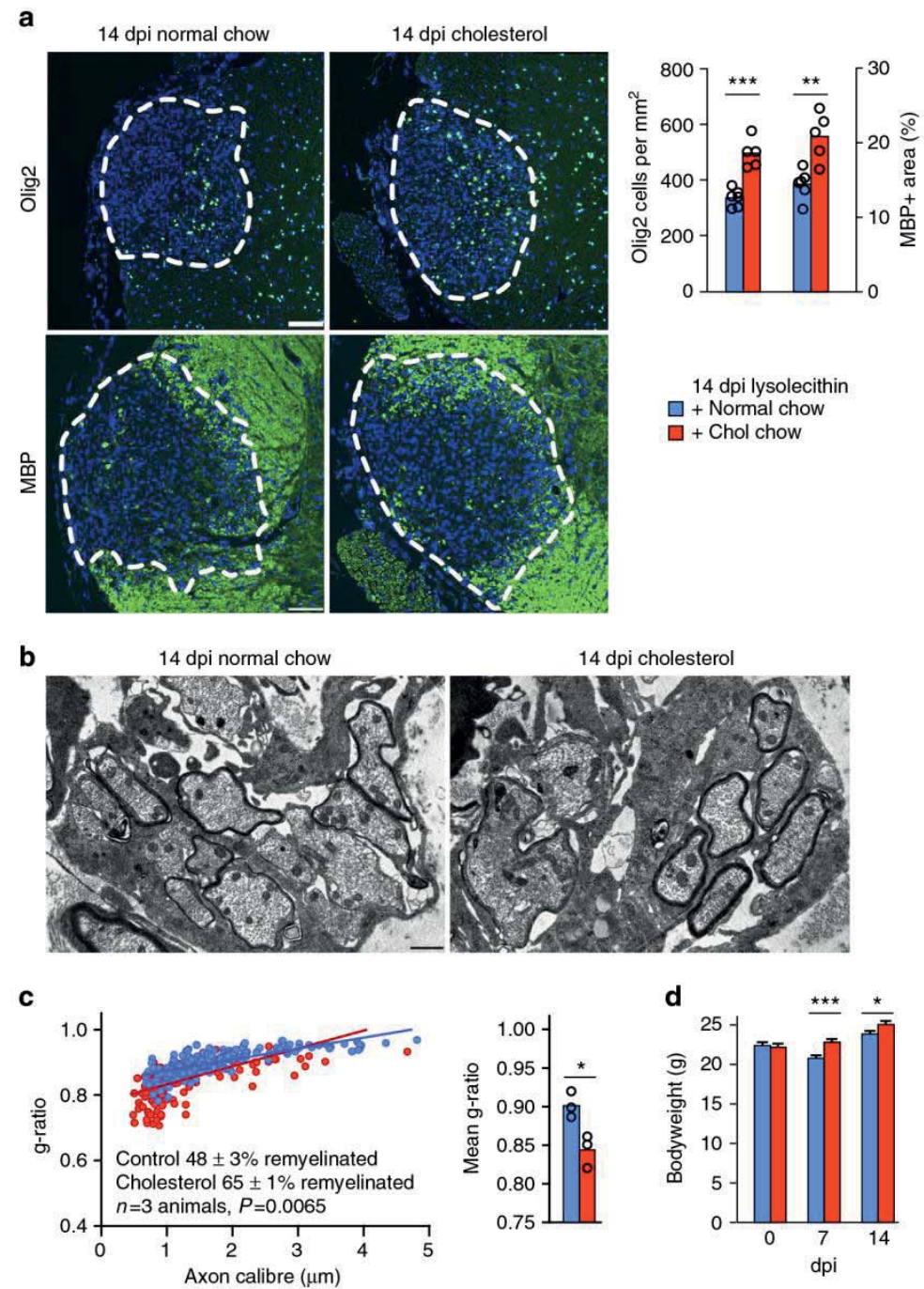

Figure 6 | Cholesterol supports remyelination in the lysolecithin model. (a) Representative images of spinal cord sections 14 days post lesion (dpl) with $1 \mu \mathrm{l} 1 \%$ lysolecithin in the ventral spinal cord with quantification of $n=5$ (cholesterol chow) and $n=6$ (normal chow) animals. Student's $t$-tests revealed significantly more Olig2 positive oligodendroglial cells within the lesion area $(P<0.0001)$, and significantly more MBP positive area $(P=0.027$; scales, $100 \mu \mathrm{m}) .(\mathbf{b}, \mathbf{c})$ Representative electron micrographs (scale $1 \mu \mathrm{m}$ ) and quantification of myelin sheath thickness and the portion of remyelinated axons in control and cholesterol fed mice at $14 \mathrm{dpl}$ by g-ratio analysis ( $n=3$ animals per group). (d) Body weight of experimental animals assessed at the day of lesion (day 0), after $7 \mathrm{~d}$ and after $14 \mathrm{~d}$ at the end of the experiment. Shown are the means \pm s.e.m. of $n=9$ (chol chow) to 10 (normal chow) animals. Twoway ANOVA with Sidaks post tests revealed a significant influence of cholesterol feeding at both time points $(7 \mathrm{dpi} P<0.0003,14 \mathrm{dpi} P=0.0362)$.

(Fig. 7b, Supplementary Fig. 8b); neither the final degree of neurite outgrowth ${ }^{35}$ nor myelination, were influenced by cholesterol. These findings suggest that external cholesterol directly facilitates oligodendrocyte differentiation and the synthesis of myelin membranes.

In principle, a substantial induction of OPC differentiation could be unfavourable, if it occurs at the expense of OPC numbers. Indeed, gradual depletion of OPCs was observed in cholesterol supplemented oligodendroglial cultures (Fig. 7a, left panel). Thus, the expansion of proliferative OPCs in vivo (Fig. 5c) is likely an indirect consequence of additional factors from the local environment. To identify factors that mediate cholesterol dependent OPC proliferation, we analysed another cohort of mice in the 'induced remyelination' treatment paradigm $(4+1$ weeks), using quantitative RT-PCR on dissected corpus callosi. In agreement with our histological data, oligodendrocyte related genes were (i) strongly downregulated in cuprizone fed mice in comparison to untreated controls (grey line) and (ii) significantly enhanced in cholesterol fed animals in comparison to chow fed animals (Fig. 7c, compare Fig. 5, Supplementary Table 1). Similarly, the astrogliosis (Gfap) and diminished microgliosis (Aif1, allograft inflammatory factor 1) were also reflected in the expression levels of respective marker genes (Fig. 7d). Surprisingly, cholesterol supplementation did not lead to feedback inhibition of cholesterol synthesis, but rather, increased the expression of genes involved in cholesterol synthesis and 
a

OPC
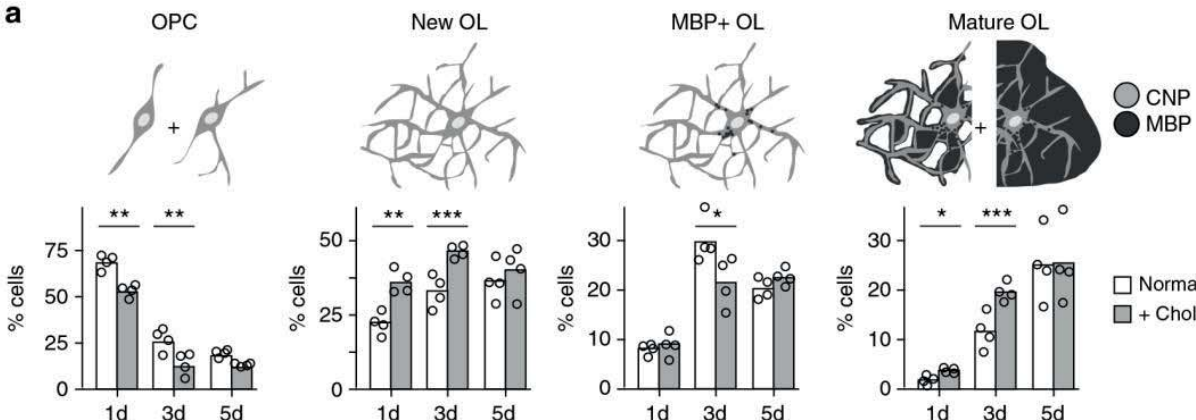

$\square$ Normal media

b
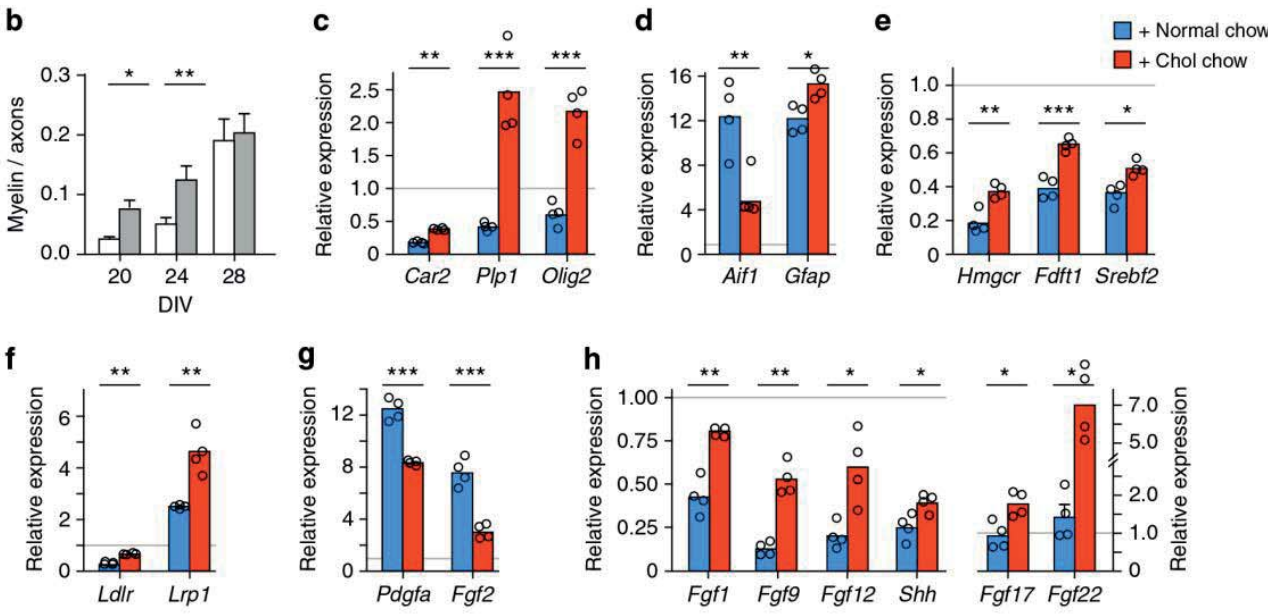

i

$$
\begin{aligned}
& \text { FGF1/FGF2 } \\
& \square 45 / 80
\end{aligned}
$$

$\square 90 / 35+\mathrm{chol}$

j

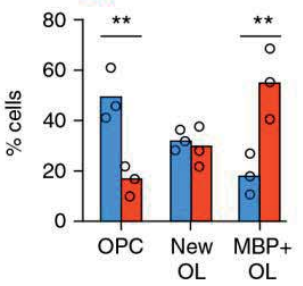

$\square$ Normal media
$\square+$ Cholesterol

\section{k $\square+$ Chol}

$\square+$ Cup

$\square+$ Cup + chol
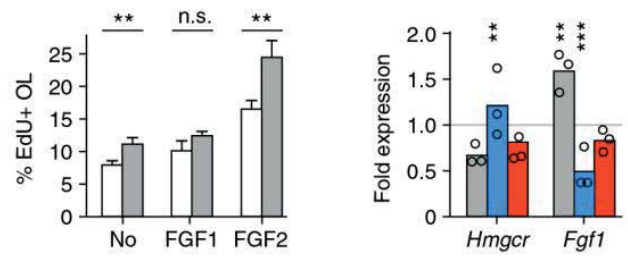

Figure 7 | Cholesterol alters the expression profile of growth factors. (a) Differentiation time course of OPCs in oligodendroglial enriched cultures in the presence or absence of cholesterol supplementation (bars represent mean of $n=4$ cultures with individual data points). Drawings illustrate chosen categories of oligodendrocyte differentiation. In each category, significance was assessed by two-way ANOVA and Sidak's post tests. (b) Myelination at 20-28 days in vitro (DIV) in myelinating cocultures in the presence or absence of cholesterol ( $n=5-9$ cultures). Myelin segments and axons were counted (see Supplementary Fig. 8b; two-way ANOVA and Sidak's post tests). (c-h) Quantitative RT-PCR analysis on dissected corpus callosi from mice after 'induced remyelination' ( $4+1$ weeks) and controls determining the expression of oligodendrocyte and myelin related genes (c; Car2, PIp1, Olig2), marker genes for microglia (Aif1) and astrocytes (Gfap) (d), genes involved in cholesterol synthesis (e; Hmgcr, Fdft1, Srebf2) and uptake (f; Ldlr, Lrp1), and growth factors downregulated (g: Pdgfa, Fgf2) and upregulated by cholesterol supplementation (h; Fgf1, Fgf9, Fgf12, Shh, Fgf17, Fgf22). Bars represent the means ( $n=4$ animals) with individual data points (Student's t tests) normalized to untreated control mice (set to 1, grey line). (i) Differentiation of rat oligodendroglial cells in cultures supplemented with FGF1 and FGF2 (concentrations in ng per $\mathrm{ml}$ as indicated) in the presence or absence of cholesterol. Bars represent mean percentage of cells in each category of $n=3$ cultures (two-way ANOVA with Sidak's post test). (j) Proliferation of OPCs in response to growth factors and cholesterol. OPCs were cultured in the presence or absence of the growth factors (100 ng ml $\left.^{-1}\right)$ FGF1 or FGF2 with or without cholesterol for $24 \mathrm{~h}$. Data are mean EdU positive cells of all oligodendroglial cells \pm s.e.m. ( $n=13$ (no GF, FGF2) or $n=7$ (FGF1) cultures of individual rats; Student's t-tests). (k) Quantitative RT-PCR on primary astrocytes treated with cuprizone (cup) with or without cholesterol (chol) supplementation. Bars represent the mean of $n=3$ independent experiments with individual data points compared with untreated cultures (set to 1, grey line; one-way ANOVA with Sidak's post tests. Asterisks represent significant differences with ${ }^{\star} P<0.05 ;{ }^{\star \star} P<0.01 ;{ }^{\star \star \star} P<0.001$. 
uptake (Fig. 7e,f), likely indicating enhanced remyelination. In contrast, expression of LXR family genes, which influence OPC differentiation ${ }^{36}$, was not affected by cholesterol (Supplementary Table 1).

The expression of growth factors involved in OPC survival, proliferation, migration or differentiation ${ }^{28}$, including Igf 1 (insulin-like growth factor), Cntf (ciliary neurotrophic factor), Inhba (inhibin beta-A, also called activin beta-A) and $E g f$ (epidermal growth factor) was strongly increased (2-20 fold) by cuprizone, but was not further regulated by cholesterol supplementation (Supplementary Table 1). A set of genes whose products are known to inhibit differentiation of OPCs, such as Fgf2 (fibroblast growth factor 2) and Pdgfa (platelet derived growth factor alpha) ${ }^{37-39}$, was also strongly upregulated by cuprizone (8-12 fold higher than untreated controls). Strikingly, the expression of these mitogens was attenuated in cholesterol fed animals to levels only 3-8 fold higher than in untreated controls (Fig. 7g). Moreover, in comparison to untreated controls, expression of another set of factors, some of which are known to facilitate differentiation of oligodendrocytes ${ }^{39,40}$, such as Fgfl and Shh (sonic hedgehog), was reduced by cuprizone, but strongly elevated by cholesterol supplementation (Fig. 7h). Expression of FGF receptors (1-3) was not influenced by cholesterol (data not shown). Demonstrating the generality of these findings (Supplementary Table 2), cholesterol influenced the profile of growth factor expression in a similar manner in mice treated chronically with cuprizone (12 weeks, see Fig. 4). In contrast to the 'induced remyelination' paradigm, expression of enzymes involved in cholesterol synthesis was reduced in this cohort, suggesting feedback inhibition after remyelination is accomplished (Supplementary Table 2).

To determine whether the growth factor expression profile observed in cholesterol treated mice might be causally related to the enhanced repair, we tested whether these growth factor combinations directly enhance OPC differentiation in vitro, a surrogate for remyelination in vivo. Indeed, differentiation was enhanced when OPCs were cultured for 3 days in media supplemented with $90 \mathrm{ng} \mathrm{ml}^{-1} \mathrm{FGF} 1,35 \mathrm{ng} \mathrm{ml}^{-1} \mathrm{FGF} 2$ and cholesterol (exemplifying cuprizone + cholesterol chow), in comparison to $45 \mathrm{ng} \mathrm{ml}^{-1}$ FGF1 and $80 \mathrm{ng} \mathrm{ml}^{-1}$ FGF2 (exemplifying cuprizone + normal chow) (Fig. $7 \mathrm{i}$ ). These data suggest the changes in growth factor expression are directly contributing to the improved repair.

Next, we cultured OPCs for $24 \mathrm{~h}$ in the presence of EdU (5-ethynyl-2'-deoxyuridine), a marker of cells in S-phase of the cell cycle, to determine whether the proliferative effect of growth factors was modified by cholesterol. Compared with vehicle treated controls, FGF2 doubled the number of EdU positive cells (as expected ${ }^{41}$ ), while FGF2 plus cholesterol elicited a threefold increase in this population (Fig. 7j), suggesting that cholesterol potentiates the effects of FGF2. Indeed, cholesterol alone only slightly increased the proportion of EdU + cells in these cultures (Fig. 7j). We speculate that, despite attenuated $\mathrm{Fg} 2$ expression (compare Fig. 7g), potentiated FGF2 signalling contributes to the expansion of proliferating OPCs in cholesterol fed animals (compare Fig. 5c).

As only relatively few microglial cells are present in the corpus callosum of cholesterol fed mice in the 'induced remyelination' paradigm $(4+1$ weeks) and in the 'chronic cuprizone' paradigm (12 weeks), we hypothesized that astrocytes contributed principally to the altered profile of growth factors. Indeed, while primary astrocytes downregulated Fgfl expression in response to cuprizone, its expression was upregulated in response to cholesterol, irrespective of cuprizone (Fig. 7k), correlating with our in vivo data. Taken together, in the cuprizone model, cholesterol supplementation modulates the expression profile of growth factors, rebalancing proliferative and differentiation signals creating a permissive environment for repair.

\section{Discussion}

Cholesterol availability is a prerequisite for myelination ${ }^{17,42,43}$ and, as we show here, exogenous cholesterol directly increases the rate of OPC differentiation. In agreement with our findings, failure to upregulate expression of sterol synthesis enzymes leads to arrested differentiation of Tcf4 mutant OPCs, which can partially be rescued by cholesterol supplementation ${ }^{44}$. Further, cholesterol synthesis is enhanced during remyelination in mice ${ }^{29}$ and statin administration (inhibitors of sterol and isoprenoid synthesis) interferes with remyelination in the cuprizone model ${ }^{45}$. Nonetheless, monotherapy with statins ameliorates clinical scores in EAE; an effect associated with decreased CNS infiltration and inflammatory activity of $\mathrm{T}$ cells, likely reducing demyelination ${ }^{46-48}$. The outcomes of studies using statins in MS patients are contradictory, probably because of the disparate effects of statins on inflammation (beneficial ${ }^{46-48}$ ) and on remyelination (detrimental ${ }^{45}$ ). Accordingly, a recent metaanalysis does not recommend statin treatment for relapsingremitting MS or clinically isolated syndrome ${ }^{49}$. Hence, we hypothesize that remyelination failure in MS reflects, at least partially, the inability to locally increase the cholesterol content in demyelinated lesions.

This hypothesis is supported by the current study. Exogenous cholesterol enters the CNS through an impaired blood-brain barrier, resulting in enhanced repair and an amelioration of the neurological phenotype in two distinct models of remyelination. Our data suggest that cholesterol directly facilitates repair by modulating the profile of growth factor expression, promoting OPC differentiation and, together with the mitogen FGF2, potentiating OPC proliferation. Importantly, cholesterol supplementation does not exacerbate inflammation in EAE.

What could prevent the increase of cholesterol in OPCs in demyelinated lesions? In patients with MS and in models of demyelination, CNS cholesterol homoeostasis is destabilized by a variety of mechanisms. First, expression of enzymes involved in cholesterol synthesis is reduced in demyelinated lesions (our study and refs 9,29,50). Second, intercellular cholesterol transport in the CNS is perturbed in patients with MS, because of reduced abundance of relevant proteins such as ApoE (Apolipoprotein E) ${ }^{10}$; and in mouse mutants with BBB disruption ${ }^{22}$, by uncontrolled flux of sterols in and out of the brain. BBB disruption has been shown by diffusion MRI in inflammatory diseases of the brain, such as MS (ref. 51) and, as we demonstrate here for the first time, is also a feature in the cuprizone model. Third, the decrease in serum cholesterol in both EAE and cuprizone mouse models, probably contributes to the impairment of CNS cholesterol homoeostasis. Whether patients with MS experience a drop in serum cholesterol during acute demyelinating episodes is unknown, and its analysis complicated by the fact that the standard first-line interferon beta treatment itself reduces total serum cholesterol ${ }^{52}$. Finally, the OPCs in chronically demyelinated lesions in $\mathrm{MS}^{50}$ and mouse models ${ }^{29}$, fail to upregulate lipid synthesis and differentiate, potentially as a consequence of an imbalance in signalling, as previously hypothesized $^{4}$. Here, we demonstrate an imbalance in expression of growth factors in the cuprizone model, in accordance with previous studies ${ }^{28,53-55}$. The growth factor profile associated with cuprizone alone, such as high levels of FGF2 and PDGFa, is predicted to facilitate OPC proliferation but impede efficient remyelination, particularly after chronic demyelination. 

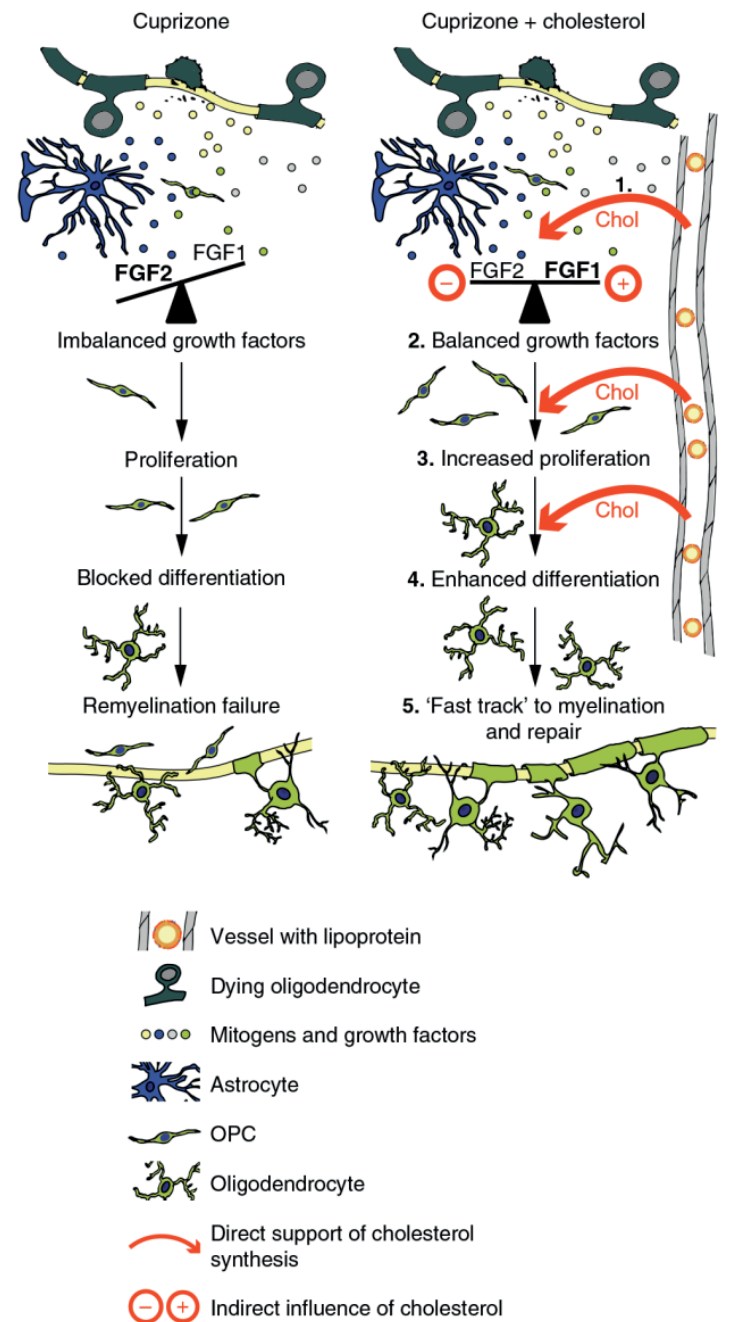

Figure 8 | Working model of repair processes influenced by cholesterol. Working model of nutritional cholesterol mediated repair processes. Cuprizone exposure causes oligodendrocyte loss and demyelination and slow repair (left panel) because of OPC depletion, imbalanced growth factors, and low local availability of cholesterol. In case of nutritional supplementation, cholesterol from the circulation enters the CNS because of increased BBB permeability (red arrows) increasing the local cholestero availability (1). There, cholesterol rebalances the expression of growth factors and mitogens synthesized e.g. by astrocytes (2). This simultaneously enhances OPC proliferation (3) and opens a window for OPC differentiation. Cholesterol directly facilitates oligodendrocyte differentiation, presumably by relieving cells from time and energy intensive cholesterol synthesis (4). Altogether, these effects provide a 'fast track' to remyelination and repair (5)

Specifically, FGF signalling could critically influence the fate of demyelinated lesions $s^{39,53,56,57}$. FGF signalling comprises a very complex network, including 24 FGF family members and four different receptors, whose signalling outcome depends on various splice isoforms and on the multifaceted crosstalk between different pathways ${ }^{58}$. Here, we focused on the two major FGF members involved in myelination, FGF1 and FGF2. FGF2 has been implicated in OPC proliferation, migration and inhibition of oligodendrocyte differentiation $38,39,41$. In the cuprizone model and in patients with MS, FGF2 abundance correlates with the degree of OPC proliferation ${ }^{28,53,56,59}$. FGF2 is increased in regions of active OPC proliferation and ongoing remyelination, such as active lesions or the rim of demyelinated lesions, while it is downregulated in remyelinated shadow plaques, and it is low in abundance in normal appearing white matter and in the core of demyelinated silent lesions ${ }^{59}$. We show that cholesterol administration attenuates the overexpression of $\mathrm{Fg} 22$ and other mitogens in the cuprizone model. Surprisingly, this did not restrict proliferation but augmented OPC numbers, likely through synergy with cholesterol (see below).

FGF1 is reduced in active MS lesions ${ }^{60}$, and increased expression is only found in remyelinated lesions ${ }^{56}$. In contrast to FGF2, FGF1 is not mitogenic for OPCs (our study and refs 39,56). Rather, FGF1 accelerates myelination in vitro ${ }^{56}$, to an extent remarkably similar to what we observed in cholesterol treated cultures. FGF1 might support CNS repair by inducing lipid synthesis and secretion by astrocytes ${ }^{61}$. The crosstalk between FGF signalling and regulation of cholesterol metabolism is supported by the presence of sterol responsive elements (consensus sequences for the SREBF2 transcription factor that increases cholesterol synthesis) in the Fgf1 and Fgf2 promoters (https://www.genomatix.de/). Altered cellular cholesterol levels can modulate signalling pathways, as shown for wnt signalling; the cholesterol content regulates the recruitment of different sets of scaffolding/adaptor proteins to the plasma membrane ${ }^{62}$. We demonstrate that cholesterol induces the expression of Fgfl in astrocytes; however, the affected signalling remains enigmatic. In addition to astrocytes, other cells such as microglia, OPCs, neurons and vascular cells likely participate in growth factor synthesis. In cholesterol treated mice, the expression profile of growth factors was altered such that the mitogens FGF2 and PDGF $\alpha$ were attenuated and differentiating cues such as FGF1 and Shh were enhanced. Other factors that are unaffected by cholesterol probably also contributed to the repair process.

Our data reveal a previously unknown function of nutritional cholesterol in adult remyelination (Fig. 8 shows a working model). In response to cuprizone-mediated demyelination in mice, the secreted mitogens and growth factors favour the proliferation and oppose the differentiation of OPCs, which slows and ultimately impairs remyelination. Dietary supplementation increases cholesterol availability within the demyelinated CNS and this is associated with rebalancing of growth factor expression. The altered profile of growth factors in cholesterol treated mice simultaneously facilitates OPC proliferation and oligodendrocyte differentiation in vivo. Thus arrested repair can be overcome by increasing the local availability of cholesterol which we achieved by nutritional supplementation.

We envision that moderate concentrations of the mitogen FGF2, when in synergy with cholesterol, potentiate OPC proliferation, and at the same time opens a window for OPC differentiation that is also enhanced by increased levels of prodifferentiation factors. In addition, cholesterol might directly facilitate oligodendrocyte differentiation, by relieving cells of the burden of establishing the complex time- and energy-intensive anabolic cholesterol pathway. Supplemented cholesterol can directly support myelination by incorporation into myelin membranes, as shown previously in a leukodystrophy model ${ }^{18}$. The current study suggests that cholesterol provides a 'fast track' to remyelination and repair.

In contrast to the beneficial effect on remyelination, highcholesterol chow ( $2 \%$ cholesterol) has no effect on demyelination and oligodendrocyte survival in the cuprizone model, likely because oligodendrocyte loss is induced by direct 
Publications/Manuscripts

cuprizone-mediated damage, and is concomitant with a low-grade inflammatory cascade involving $\mathrm{T}$ cells, astrocytes and microglia ${ }^{63}$. Consistent with cholesterol supplementation not exacerbating demyelination in the cuprizone model, high-cholesterol chow (5\% cholesterol) did not aggravate disease in EAE. Moreover, cholesterol supplementation attenuated axonal damage in both models; during active remyelination in the cuprizone model and during remission in EAE. Likely, this is secondary to ameliorated disease states and balanced expression of growth factors and pro-inflammatory factors. We found that dietary cholesterol slightly ameliorated inflammation in EAE, while, in contrast, a high-fat chow aggravates EAE symptoms ${ }^{64,65}$. Medium and long chain fatty acids of the high-fat chow probably modulate $T$ cell differentiation ${ }^{66}$. Whether fatty acids contribute to the increased disease activity in some MS patients with elevated serum cholesterol remains unclear ${ }^{12}$. Importantly, feeding cholesterol appears to be safe in mice with inflammatory disease.

Taken together, our data show that demyelinating disease destabilizes peripheral and CNS cholesterol homoeostasis. Dietary cholesterol supplementation supports cholesterol metabolism in the CNS and has the remarkable potential to ameliorate disease by facilitating several repair mechanisms, leading to improved remyelination and neurological outcome. This study highlights the safety of dietary cholesterol and might have implications for the management of demyelinating diseases, but further studies, especially in combination with immune suppressive drugs, are required to determine its feasibility for patients.

\section{Methods}

Mice. All animal studies were performed in compliance with the animal policies of the Max Planck Institute of Experimental Medicine, and were approved by the German Federal State of Lower Saxony. Adult male C57BL/6N mice (8-10 weeks of age) were taken for all analyses. Animals were randomly assigned to an experimental group. Mice were fed normal chow (V1124 ssniff Spezialdiäten GmbH, Germany) or chow supplemented with either $2 \% \mathrm{w} / \mathrm{w}$ (cuprizone and lysolecithin experiments) or $5 \% \mathrm{w} / \mathrm{w}$ cholesterol (EAE experiments).

For MOG-EAE, mice purchased from Charles River were immunized subcutaneously with $200 \mu \mathrm{g}$ myelin oligodendrocyte glycoprotein peptide 35-55 (MOG35-55) in complete Freund's adjuvant (M. tuberculosis at $3.75 \mathrm{mg} \mathrm{ml}^{-1}$ ) and i.p. injected twice with $500 \mathrm{ng}$ pertussis toxin as described ${ }^{67}$. Animals were examined daily and scored for clinical signs of the disease. If disease did not start within 15 days after induction or the clinical score rose above 4 , animals were excluded from the analysis. The clinical score was: 0 normal; 0.5 loss of tail tip tone; 1 loss of tail tone; 1.5 ataxia, mild walking deficits (slip off the grid); 2 mild hind limb weakness, severe gait ataxia, twist of the tail causes rotation of the whole body; 2.5 moderate hind limb weakness, cannot grip the grid with hind paw, but able to 2.5 moderate hind limb weakness, cannot grip the grid with hind paw, but able to
stay on a upright tilted grid; 3 mild paraparesis, falls down from a upright tiled grid; 3.5 paraparesis of hind limbs (legs strongly affected, but move clearly); 4 paralysis of hind limbs, weakness in forelimbs; 4.5 forelimbs paralyzed; 5 moribund/dead. Mice received 5\% cholesterol chow commencing either two weeks before immunization defined as prophylactic regimen or at the first appearance of EAE symptoms defined as therapeutic regimen and continued until day 28.

For cuprizone experiments, mice were fed $0.2 \% \mathrm{w} / \mathrm{w}$ cuprizone (Sigma-Aldrich Inc., Germany) in powder chow with or without cholesterol for 'demyelination' (2-5 weeks) and 'chronic cuprizone' (6 and 12 weeks) paradigms. For 'induced remyelination' experiments, mice were fed cuprizone in standard chow for 4 weeks, followed by cuprizone withdrawal and feeding mice standard chow with or without cholesterol supplementation. Mice were fed three times a week an exceeding amount of chow by dispenser. Food intake and animal weight was monitored. Age-matched untreated controls were fed standard powder chow.

Focal spinal cord demyelinating lesions were induced under anaesthesia by stereotactic injection of $1 \mu \mathrm{l}$ lysolecithin ( $1 \%$, from egg yolk, alpha-

lysophosphatidylcholine, Sigma) into the ventro-lateral funiculus at Th10 of 8 -week old animals, as previously described ${ }^{68}$. The injection was performed with a $10 \mu \mathrm{l}$ Hamilton syringe, fitted with a thin tapered glass tip, at a rate of $\sim 1 \mu \mathrm{l} \mathrm{min}{ }^{-1}$. This procedure created fusiform demyelinating lesions, $5-6 \mathrm{~mm}$ in length. At the day of injection, mice were randomly assigned to normal or $2 \%$ cholesterol chow for 14 days, after which the animals were killed, and the spinal cord processed for histology.

Bodipy-cholesterol injections were done as described ${ }^{18}$. Briefly, bodipycholesterol (Topflour, Avanti Polar Lipids) was injected i.p. ( $16 \mu \mathrm{gg}^{-1}$ body weight). After one week, mice were perfused, and bodipy-cholesterol fluorescence was analysed on vibratome sections using a custom made two-photon laser scanning microscope equipped with a titanium-sapphire laser and a $\times 20$ water immersion objective (NA 1.0). Z-stacks of $100-\mu \mathrm{m}$ depth were obtained and processed to maximum intensity projections. For tracer quantification of bodipy-cholesterol ( $5 \mathrm{\mu gg}^{-1}$ body weight, i.p. injection $7 \mathrm{~d}$ circulation time) or Evans blue ( $50 \mu \mathrm{gg}^{-1}$ body weight, i.v. injection, $4 \mathrm{~h}$ circulation time) animals were perfused with PBS to remove tracer from the circulatory system. Brains were dissected and immediately frozen on dry ice, weighed and stored at $-80^{\circ} \mathrm{C}$ for further processing. Tissue was lyophilized (Christ LMC-1 BETA 1-16) at $-36^{\circ} \mathrm{C}$ for $24 \mathrm{~h}$ under vacuum of $0.2 \mathrm{mBar}$. For tracer extraction, hemispheres were incubated shaking in $10 \mu \mathrm{l}$ formamide per mg brain at $57^{\circ} \mathrm{C}$ for $24 \mathrm{~h}$. Integrated density of tracer fluorescence was determined in triplicates on a fluorescent microscope (Observer Z2, Zeiss, Germany), equipped with an AxioCam MRc3, × 1 Camera Adaptor and the ZEN 2012 blue edition software recorded at $\times 10$ magnification (Plan-Apochromat $\times 10 / 0.45 \mathrm{M} 27$ ). Tracer concentration was calculated using a standard curve and normalized to matched controls (set to 1).

Motor skill performance was assessed essentially as described ${ }^{34}$. Mice were randomly divided into two treatment and two control groups $(n=6-13)$ and housed in individual cages that allow computer-controlled recording of wheel rotation as a function of time (MatLab-based custom software). The axis of each wheel was attached to a rotation sensor with a resolution of 16 per turn. One wheel revolution comes up to a running distance of $35.5 \mathrm{~cm}$. The running wheel revolutions were recorded continuously at a sampling rate of $1 / 0.48 \mathrm{~s}$ by a customized recording device and software (Boenig \& Kallenbach oHG, Dortmund, Germany). Mice in treatment groups were treated as in the 'induced remyelination' paradigm (feeding 4 weeks cuprizone in normal chow followed by withdrawal of cuprizone and feeding normal chow or cholesterol supplemented chow). Control animals received normal chow for the entire experiment or were switched to

cholesterol chow after 4 weeks. One week after the start of the experiment training wheels with regularly spaced rungs were placed into the cages for adaptation of cardiopulmonary and musculoskeletal strength. One week after the switch of diets (week 5 of experiment), wheels were replaced by complex wheels with irregularly spaced rungs to assess the bilateral sensorimotor coordination that likely involves the cerebellum and motor cortex and connecting white matter such as the corpus callosum. Specifically, we measured maximum running velocity $\left(\mathrm{V}_{\max }\right)$, in addition to the total maximum run duration $\left(\mathrm{D}_{\max }\right)$, accumulative distance in metres (Dist $t_{a c}$ and the number of individual runs $\left(\mathrm{N}_{\mathrm{run}}\right)$. Parameters were logged once daily $(12 \mathrm{am})$

Serum analyses. Animals were fasted for $4 \mathrm{~h}$, blood was collected from the retroorbital sinus, and serum was prepared after clotting by centrifugation. Cholesterol measurements were done with the architectII system (Abbott Diagnostics).

Cell isolation and flow cytometry. Single-cell suspensions from spinal cords were Cell isolation and flow cytometry. Single-cell suspensions from spinal cord
obtained via mechanical dissociation on a cell strainer. Immune cells were obtained via mechanical dissociation on a cell strainer. Immune cells were
separated over a two-phase Percoll-density gradient. Staining of $\alpha \beta T C R / C D 4$ $\mathrm{T}$ cells, $\alpha \beta T C R / C D 8^{+} \mathrm{T}$ cells and CD45/CD11b cells (macrophages/microglia) was performed using the following antibodies in a 1:200 dilution: Anti-CD3e (clone 145-2C11), BioLegend; anti-CD4 (clone GK 1.5), BD; anti-CD8 (clone 53-6.7), BD; anti-CD8 (clone 53-6.7), BD; anti-CD11b (clone M1/70), BioLegend; anti-CD45.2 (clone 104), BioLegend. The addition of Calibrite APC beads (BD) allowed for cell quantification. Flow cytometry was performed using a FACSCalibur operated by Cell Quest software (Becton Dickinson).

Histochemistry. Anesthetized mice were perfused with $4 \%$ formaldehyde (PFA). Brain samples of cuprizone treated animals were cut at Bregma 1.58 for comparable pathology because the extent of cuprizone mediated demyelination strongly depends on the rostral/caudal position ${ }^{69}$. Tissue was postfixed overnight, embedded in paraffin and cut into $5 \mu \mathrm{m}$ sections (HMP 110, MICROM). Gallyas silver impregnation was done as described ${ }^{18}$. For immunohistological analyses, sections were deparaffinized followed by antigen-retrieval in sodium citrate buffer $(0.01 \mathrm{M}, \mathrm{pH} 6.0)$. For immunofluorescence, sections were blocked with serum free protein block (Dako). Primary antibodies were diluted in $2 \%$ bovine serum albumin (BSA)/PBS and incubated for $48 \mathrm{~h}$ followed by fluorophor coupled secondary antibodies. For immunohistochemistry, endogenous peroxidase activity was blocked with $3 \%$ hydrogen peroxide. Sections were then blocked ( $20 \%$ goat serum in BSA/PBS) and incubated with primary antibodies. Detection was done with the LSAB2 kit (Dako, Hamburg, Germany) or the Vector Elite ABC kit (Vector Labs). HRP substrate 3,3'-Diaminobenzidine (DAB) was applied by using the DAB Zytomed Kit (Zytomed Systems $\mathrm{GmbH}$ ). Haematoxylin stain was done to label nuclei. Sections were dehydrated before mounting (Eukitt). Specimens were analysed on an Axio Imager.Z1 (Zeiss) equipped with an AxioCam MRc3, $\times 0.63$ Camera Adaptor and the ZEN 2012 blue edition software using $\times 10$ objective (Plan Apochromat $\times 10 / 0.45$ M27) or $\times 20$ objective (Plan-Apochromat $\times 20 / 0.8$ ) and evaluated with Image J software. Quantification of areas (Gallyas, GFAP, MAC3) were done by applying semi-automated ImageJ software macro to threshold (variable threshold in case of Gallyas and fixed threshold for antibody stainings) and colour deconvolute the images of the corpus callosum above the fornix (Bregma 1.58). Three to five sections per animal were analysed. Quantification of EAE lumbar spinal cord lesions was done on two to fou 
quadruple stained sections (Ibal (induction of brown adipocytes 1), CD3, GFAP, DAPI) per animal recorded with tile region setup and shading correction. Lesion area was defined by focal accumulation of at least 20 DAPI positive cells, the presence of microglia and infiltration of $\mathrm{CD} 3$ positive cells. Lesion area, number of Iba1 and $\mathrm{CD} 3$ positive cells and GFAP positive area were evaluated. Quantification of cuprizone treated animals: cell number (CAII (carbonic anhydrase 2), Olig-2, TCF4, PCNA), APP positive spheroids and area (Gallyas, GFAP, MAC3) was done in the corpus callosum above the fornix (Bregma 1.58). Three to five sections per animal were analysed. Microscope settings are listed in Supplementary Table 4.

Electron microscopic analysis was done as previously described ${ }^{17}$. Briefly, tissue was fixed in 4\% PFA, $2.5 \%$ Glutaraldehyde, $0.1 \mathrm{~m}$ Phosphate buffer and sagittal sections were cut on a vibratome (Leica VT1200, $300 \mu \mathrm{m}$ ). The corpus callosum with adjacent tissue $(-0.04 \mathrm{~mm}$ lateral) was punched with a $2 \mathrm{~mm}$ diameter punching tool and embedded in epon (EMTP, Leica). At least 15 digital pictures ( $\times 12,000$ magnification, TRS, Moorenweis) of uranyl acetate contrasted ultrathin sections were taken with the Zeiss EM900.

Antibodies. The following antibodies were used: APP (Chemicon MAB348), CAII (Said Ghandour); CD3 (Serotec MCA1477);CD3e (Biolegend clone 145-2C11), CD4 (Becton Dickinson clone GK1.5), CD8 (Becton Dickinson clone 53-6.7), CD11b (Biolegend clone M1/70), CD45.2 (Biolegend clone 104), CNP $\left(2^{\prime}, 3^{\prime}\right.$-Cyclic-nucleotide $3^{\prime}$-phosphodiesterase, Sigma C5922), GFAP (Chemicon MAB3402), Iba1 (Wako 019-19741), MAC3 (Pharmigen 01781D); MBP (Serotec MCA409S), Olig2 (Prof Charles Stiles/ Dr. John Alberta, DF308), PCNA (Abcam ab29), SMI31 (Covance SMI-31P), TCF4 (Millipore 04-1080). Expression analyses. For the characterization of the proinflammatory milieu in
EAE mice, RNA from total spinal cord lysates was isolated using Trizol (Thermo EAE mice, RNA from total spinal cord lysates was isolated using Trizol (Ther
Fisher). complementary DNA (cDNA) was synthesized using RevertAid First Strand cDNA Synthesis Kit (Thermo Fisher) according to the manufactureŕs protocol. Quantitative RT-PCR was performed using a StepOnePlus Real-Time PCR System operated by StepOnePlus Software v2.0. Target-specific FAM- and TAMRA-labeled TaqMan probes were used in all cases. Measurements were performed in independent duplicates. Gene expression was normalized to $\beta$-actin. Relative changes in gene expression were analysed via the $2 \Delta \Delta C(T)$ method.

For expression analyses on brain sections, mice were killed by cervical For expression analyses on brain sections, mice were killed by cervical
dislocation and brains were quickly cooled and sliced coronally using a brain dislocation and brains were quickly cooled and sliced coronally using a brain
matrix (Asi-Instruments). The corpus callosum was dissected from Bregma +1.10 to -2.46 and RNA was extracted using RNeasy Mini (Qiagen). The concentratio and quality of RNA was evaluated using a NanoDrop spectrophotometer and RNA Nano (Agilent). cDNA was synthesized with Superscript III (Invitrogen) and quantitative PCRs were done in triplicates with the GoTaq master mix (Promega) on a 7500 Fast Real-Time PCR System (Applied Biosystems). Expression values were normalized to the geometric mean of two housekeeping genes, Hprt (Hypoxanthin-Phosphoribosyl-Transferase 1) and Rplp0 (60S acidic ribosomal protein $\mathrm{P}$ ) and analysed by the $\Delta \Delta C t$ method.

Expression of the following genes was measured: Abcal (ATP-binding cassette transporter A1), Actb (beta actin), Aif1 (allograft inflammatory factor 1), Apoe (apolipoprotein E), Bdnf (Brain-derived neurotropic factor), Bmp2 and Bmp4 (Bone morphogenic protein 2 and 4), Car2 (carbonic anhydrase 2), Ch25h (Cholesterol 25-Hydroxylase ), Cntf (ciliary neurotrophic factor), Cyp27al (Sterol 27-hydroxylase ), Cyp46a1 (Cholesterol 24-hydroxylase), Cyp51a1 (Sterol 14 alphademethylase), Dhcr24 (24-Dehydrocholesterol reductase), Egf (epidermal growth factor), Fdft1 (Farnesyl-Diphosphate Farnesyltransferase 1), members of the $\mathrm{Fg}$ (fibroblast growth factor) gene family, Gfap (glial fibrillary acidic protein), Gmcs (granulocyte-macrophage colony-stimulating factor, CSF2), H2-DMb2 (MHCII, major histocompatibility complex class II), Hmgcr (3-Hydroxy-3-MethylglutarylCoA Reductase), Hmgcs1 (3-Hydroxy-3-methylgutaryl-CoA synthase 1), Ifng (interferon gamma), Igfl (insulin-like growth factor 1), Il2 (interleukin 2), Ill7, Inhba (inhibin beta-A, also called activin beta-A), Ldlr (Low density lipoprotein Inhba (inhibin beta-A, also called activin beta-A), Ldlr (Low density lipoprot
receptor), $\operatorname{Lrp} 1$ (Low density lipoprotein receptor-related protein 1), $M v k$ receptor), $\operatorname{Lrp} 1$ (Low density lipoprotein receptor-related protein 1), Mvk
(Mevalonate kinase), Ntf3 (Neurotrophin 3), Ngf (Nerve growth factor ), Nr1h3 (Mevalonate kinase), Ntf3 (Neurotrophin 3), Ngf (Nerve growth factor ), NrIh3
(Liver X receptor alpha, LXR alpha), NrIh2 (Liver X receptor beta, LXR beta), Olig2 (oligodendrocyte lineage transcription factor 2), Pdgfa (platelet derived growth factor alpha), Plp1 (proteolipid protein 1), Ptn (Pleiotrophin), Rxrg (Retinoic X receptor gamma, RXR gamma), $S 100 b$ (S100 calcium-binding protein B), Shh (sonic hedgehog), $\operatorname{Srbf2}$ (sterol regulatory element binding transcription factor 2), Tnf (tumour necrosis factor), Vldlr (Very low density lipoprotein receptor). All primer sequences are listed in Supplementary Table 3.

Cell cultures. For primary oligodendrocyte cultures, dissected cortices of newborn mice or rats were digested in $0.25 \mathrm{mg} \mathrm{ml}^{-1}$ Trypsin/EDTA for $10 \mathrm{~min}$ followed by triturating and plating in plating media (DMEM $4.5 \mathrm{gl}^{-1}$ Glucose, $10 \%$ fetal calf serum containing about $300 \mathrm{\mu g} \mathrm{ml}^{-1}$ cholesterol, GlutaMAX, penicillin/streptomycin). About 14 days after plating, OPCs were isolated by differential shaking an lectin panning, and plated in differentiating Sato media (DMEM $4.5 \mathrm{gl}^{-1}$

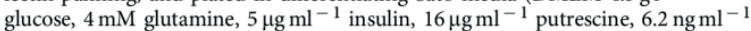
progesterone, $5 \mathrm{ng} \mathrm{ml}^{-1}$ sodium selenite, $400 \mathrm{ng} \mathrm{ml}^{-1}$ L-thyroxine, $400 \mathrm{ng} \mathrm{ml}^{-1}$ triiodothyroxine, $50 \mu \mathrm{g} \mathrm{ml}^{-1}$ holo-transferrin, penicillin/streptomycin, lacking any cholesterol source). Cell purity was routinely determined by immune stainings and always exceeded $95 \%$. Cholesterol $\left(10 \mu \mathrm{gml}^{-1}\right)$ was added from a $10 \mathrm{mg} \mathrm{ml}^{-1}$ stock solution in ethanol. Control cultures received $0.1 \%$ ethanol. In case of growth factor supplementation assays, cells were allowed to adhere for $2 \mathrm{~h}$ before treatment with EdU (5-ethynyl-2' -deoxyuridine, $10 \mu \mathrm{M}$, Invitrogen) and growth factors (FGF1, FGF2; Peprotech) at $100 \mathrm{ng} \mathrm{ml}^{-1}$ for proliferation experiments or $45 \mathrm{ng} \mathrm{ml}^{-1}$ FGF1 plus $80 \mathrm{ng} \mathrm{ml}^{-1}$ FGF2 or $90 \mathrm{ng} \mathrm{ml}^{-1}$ FGF1 plus $35 \mathrm{ng} \mathrm{ml}^{-1}$ FGF2 for differentiation experiments. Cultures were fixed with PFA and permeabilized with $0.5 \%$ Triton X100.

Myelinating co-cultures were established as described ${ }^{70}$ with minor modifications. Briefly, six E13 embryonic spinal cords per culture were digested in $0.125 \%$ Trypsin solution in HBSS (without $\mathrm{Ca}^{+2}$ and $\mathrm{Mg}^{+2}$ ) at $37^{\circ} \mathrm{C}$ for $20 \mathrm{~min}$. After stopping the digestion with $1 \mathrm{ml}$ plating media (DMEM, 25\% horse serum, $25 \%$ HBSS, $50 \mu \mathrm{g} \mathrm{ml}^{-1}$ DNAse) the tissue was homogenized by gentle trituration and centrifuged for $5 \mathrm{~min}$. 150,000 cells were plated per poly-L-lysine coated coverslip; 3 coverslips per $35 \mathrm{~mm}$ Petri dish. After cell attachment in plating media, differentiation media was added (low glucose DMEM, $10 \mu \mathrm{g} \mathrm{ml}^{-1}$ insulin,

differentiation media was added (low glucose DMEM, $10 \mu \mathrm{g} \mathrm{ml}^{-1}$ insulin,
$10 \mathrm{ng} \mathrm{ml}^{-1}$ biotin, $50 \mathrm{nM}$ hydrocortisone, $0.5 \% \mathrm{~N} 1$-mix). N1 mix was $1 \mathrm{mg} \mathrm{ml}^{-1}$ apo-transferrin, $20 \mathrm{mM}$ putrescine, $4 \mu \mathrm{M}$ progesterone, and $6 \mu \mathrm{M}$ sodium selenite. $50 \%$ media change was performed every $24-48 \mathrm{~h}$ with differentiation media. After 12 days, insulin was removed from differentiation media. Coverslips were fixed with PFA after 20,24 and 28 days in culture and permeabilized with $-20^{\circ} \mathrm{C}$ methanol for $10 \mathrm{~min}$.

Fixed and permeabilized cells were blocked with $10 \%$ horse serum in PBS and incubated with primary antibodies in blocking solution followed by secondary antibodies together with click-it kit for detection of EdU and DAPI for nuclear staining. Coverslips were mounted on slides with aqua polymount. On five randomly chosen visual fields of primary oligodendrocyte cultures $(\times 10$ magnification), stainings were evaluated. For differentiation of oligodendrocytes, $\mathrm{CNP}$ and MBP positive cells were categorized according to morphological criteri (see Fig. 7a). New oligodendrocytes (new OL) were CNP-positive MBP-negative cells with complex processes. MBP + OL cells contained few MBP-positive intracellular spots but did not form sheaths. Mature oligodendrocytes were intracellular spots but did not form sheaths. Mature oligodendrocytes were (SMI31) area and the area with myelin sheaths (MBP) of seven randomly chosen visual fields of myelinating co-cultures ( $\times 10$ magnification) was measured after binarization of thresholded images. Specimens were analysed on an Axiophot observer.Z1 (Zeiss) equipped with an AxioCam MRm and the ZEN 2012 blue edition software and evaluated with Image J software. Microscope settings are listed in Supplementary Table 4.

Statistical analyses. Statistical evaluation was done by unpaired Student's $t$-test for pairwise comparisons or by ANOVA for comparisons of more than two groups as pairwise comparisons or by ANOVA for comparisons of more than two groups a
stated in the figure legends. Two-way ANOVA was combined with a post test to evaluate individual groups. For all statistical tests, significance was measured against an alpha value of 0.05 . All error bars show s.e.m. $P$ values are shown as ${ }^{\star} P<0.05$ ${ }^{*} P<0.01 ;{ }^{* * *} P<0.001$. No statistical methods were used to predetermine sample
sizes, but our sample sizes are similar to those reported in previous publications ${ }^{25,28}$ Data analysis was performed blind to the experimental groups.

Data availability. All data generated or analysed during this study are included in this published article (and its supplementary information files) or available from the authors on request.

\section{References}

1. Irvine, K. A. \& Blakemore, W. F. Remyelination protects axons from demyelination-associated axon degeneration. Brain 131, 1464-1477 (2008).

2. Moyon, S. et al. Demyelination causes adult CNS progenitors to revert to an immature state and express immune cues that support their migration. J. Neurosci. 35, 4-20 (2015)

3. Kuhlmann, T. et al. Differentiation block of oligodendroglial progenitor cells as a cause for remyelination failure in chronic multiple sclerosis. Brain 131, $1749-1758$ (2008)

4. Franklin, R. J. \& ffrench-Constant, C. Remyelination in the CNS: from biology to therapy. Nat. Rev. Neurosci. 9, 839-855 (2008).

5. Rodgers, J. M., Robinson, A. P. \& Miller, S. D. Strategies for protecting oligodendrocytes and enhancing remyelination in multiple sclerosis. Discov. Med. 16, 53-63 (2013).

6. Harsan, L. A. et al. Recovery from chronic demyelination by thyroid hormone therapy: myelinogenesis induction and assessment by diffusion tensor magnetic resonance imaging. J. Neurosci. 28, 14189-14201 (2008).

7. Skripuletz, T. et al. Pivotal role of choline metabolites in remyelination. Brain 138, 398-413 (2015).

8. de la Fuente, A. G. et al. Vitamin D receptor-retinoid X receptor heterodimer signaling regulates oligodendrocyte progenitor cell differentiation. J. Cell Bio 211, 975-985 (2015).

9. Huang, J. K. et al. Retinoid X receptor gamma signaling accelerates CNS remyelination. Nat. Neurosci. 14, 45-53 (2011). 
10. Vuletic, S. et al. Cerebrospinal fluid apolipoprotein E and phospholipid transfer protein activity are reduced in multiple sclerosis; relationships with the brain MRI and CSF lipid variables. Mult. Scler. Relat. Disord. 3, 533-541 (2014).

11. Teunissen, C. E. et al. Decreased levels of the brain specific $24 \mathrm{~S}$ hydroxycholesterol and cholesterol precursors in serum of multiple sclerosis patients. Neurosci. Lett. 347, 159-162 (2003).

12. Giubilei, F. et al. Blood cholesterol and MRI activity in first clinical episode suggestive of multiple sclerosis. Acta Neurol. Scand. 106, 109-112 (2002).

13. Weinstock-Guttman, B. et al. Lipid profiles are associated with lesion formation over 24 months in interferon-beta treated patients following the first demyelinating event. J. Neurol. Neurosurg. Psychiatry 84, 1186-1191 (2013).

14. Meyers, L. et al. A role for Apolipoprotein A-I in the pathogenesis of multiple sclerosis. J. Neuroimmunol. 277, 176-185 (2014)

15. Tettey, P. et al. An adverse lipid profile is associated with disability and progression in disability, in people with MS. Mult. Scler. 20, 1737-1744 (2014)

16. Tall, A. R. \& Yvan-Charvet, L. Cholesterol, inflammation and innate immunity. Nat. Rev. Immunol. 15, 104-116 (2015).

17. Saher, G. et al. High cholesterol level is essential for myelin membrane growth. Nat. Neurosci. 8, 468-475 (2005)

18. Saher, G. et al. Therapy of Pelizaeus-Merzbacher disease in mice by feeding a cholesterol-enriched diet. Nat. Med. 18, 1130-1135 (2012).

19. Raddatz, B. B. et al. Central Nervous System Demyelination and Remyelination is Independent from Systemic Cholesterol Level in Theiler's Murine Encephalomyelitis. Brain Pathol. 26, 102-119 (2016).

20. Suzuki, K. Giant hepatic mitochondria: production in mice fed with cuprizone Science 163, 81-82 (1969).

21. Abbott, N. J. et al. Structure and function of the blood-brain barrier. Neurobiol. Dis. 37, 13-25 (2010).

22. Saeed, A. A. et al. Effects of a disrupted blood-brain barrier on cholesterol homeostasis in the brain. J. Biol. Chem. 289, 23712-23722 (2014).

23. Bakker, D. A. \& Ludwin, S. K. Blood-brain barrier permeability during Cuprizone-induced demyelination. Implications for the pathogenesis of immune-mediated demyelinating diseases. J. Neurol. Sci. 78, 125-137 (1987).

24. Kondo, A., Nakano, T. \& Suzuki, K. Blood-brain barrier permeability to horseradish peroxidase in twitcher and cuprizone-intoxicated mice. Brain Res. 425, 186-190 (1987)

25. Matsushima, G. K. \& Morell, P. The neurotoxicant, cuprizone, as a model to study demyelination and remyelination in the central nervous system. Brain Pathol. 11, 107-116 (2001).

26. Boretius, S. et al. Assessment of lesion pathology in a new animal model of MS by multiparametric MRI and DTI. Neuroimage 59, 2678-2688 (2012).

27. Holtta-Vuori, M. et al. BODIPY-cholesterol: a new tool to visualize sterol trafficking in living cells and organisms. Traffic 9, 1839-1849 (2008).

28. Gudi, V. et al. Spatial and temporal profiles of growth factor expression during CNS demyelination reveal the dynamics of repair priming. PLOS ONE 6, e22623 (2011).

29. Jurevics, H. et al. Alterations in metabolism and gene expression in brain regions during cuprizone-induced demyelination and remyelination. $J$. Neurochem. 82, 126-136 (2002).

30. Mason, J. L. et al. Oligodendrocytes and progenitors become progressively depleted within chronically demyelinated lesions. Am. J. Pathol. 164, 1673-1682 (2004)

31. Lürbke, A. et al. Limited TCF7L2 expression in MS lesions. PLOS ONE 8, e72822 (2013).

32. Hammond, E. et al. The Wnt Effector Transcription Factor 7-Like 2 Positively Regulates Oligodendrocyte Differentiation in a Manner Independen of Wnt/beta-Catenin Signaling. J. Neurosci. 35, 5007-5022 (2015).

33. Fancy, S. P. et al. Dysregulation of the Wnt pathway inhibits timely myelination and remyelination in the mammalian CNS. Genes Dev. 23, 1571-1585 (2009).

34. Liebetanz, D. \& Merkler, D. Effects of commissural de- and remyelination on motor skill behaviour in the cuprizone mouse model of multiple sclerosis. Exp. Neurol. 202, 217-224 (2006).

35. Fünfschilling, U. et al. Critical time window of neuronal cholesterol synthesis during neurite outgrowth. J. Neurosci. 32, 7632-7645 (2012).

36. Courtney, R. \& Landreth, G. E. LXR regulation of brain cholesterol: from development to disease. Trends Endocrinol. Metab. 27, 404-414 (2016).

37. Woodruff, R. H., Fruttiger, M., Richardson, W. D. \& Franklin, R. J. Plateletderived growth factor regulates oligodendrocyte progenitor numbers in adult $\mathrm{CNS}$ and their response following CNS demyelination. Mol. Cell Neurosci. 25, 252-262 (2004)

38. Murtie, J. C., Zhou, Y. X., Le, T. Q., Vana, A. C. \& Armstrong, R. C. PDGF and FGF2 pathways regulate distinct oligodendrocyte lineage responses in experimental demyelination with spontaneous remyelination. Neurobiol. Dis. 19, 171-182 (2005)

39. Lindner, M. et al. Fibroblast growth factor signalling in multiple sclerosis: inhibition of myelination and induction of pro-inflammatory environment by FGF9. Brain 138, 1875-1893 (2015).
40. Wu, M. et al. Differential modulation of the oligodendrocyte transcriptome by sonic hedgehog and bone morphogenetic protein 4 via opposing effects on histone acetylation. J Neurosci. 32, 6651-6664 (2012)

41. Fortin, D., Rom, E., Sun, H., Yayon, A. \& Bansal, R. Distinct fibroblast growth factor (FGF)/FGF receptor signaling pairs initiate diverse cellular responses in the oligodendrocyte lineage. J. Neurosci. 25, 7470-7479 (2005).

42. Jurevics, H. \& Morell, P. Cholesterol for synthesis of myelin is made locally, not imported into brain. J. Neurochem. 64, 895-901 (1995).

43. Mathews, E. S. et al. Mutation of 3-hydroxy-3-methylglutaryl CoA synthase I reveals requirements for isoprenoid and cholesterol synthesis in oligodendrocyte migration arrest, axon wrapping, and myelin gene expression. J. Neurosci. 34, 3402-3412 (2014).

44. Zhao, C. et al. Dual regulatory switch through interactions of Tcf712/Tcf4 with stage-specific partners propels oligodendroglial maturation. Nat. Commun. 7, 10883 (2016).

45. Miron, V. E. et al. Statin therapy inhibits remyelination in the central nervous system. Am. J. Pathol. 174, 1880-1890 (2009).

46. Youssef, S. et al. The HMG-CoA reductase inhibitor, atorvastatin, promotes a Th2 bias and reverses paralysis in central nervous system autoimmune disease. Nature 420, 78-84 (2002)

47. Stanislaus, R., Singh, A. K. \& Singh, I. Lovastatin treatment decreases mononuclear cell infiltration into the CNS of Lewis rats with experimental allergic encephalomyelitis. J. Neurosci. Res. 66, 155-162 (2001).

48. Greenwood, J. et al. Lovastatin inhibits brain endothelial cell Rho-mediated lymphocyte migration and attenuates experimental autoimmune encephalomyelitis. FASEB J. 17, 905-907 (2003).

49. Pihl-Jensen, G., Tsakiri, A. \& Frederiksen, J. L. Statin treatment in multiple sclerosis: a systematic review and meta-analysis. CNS Drugs 29, 277-291 (2015).

50. Lock, C. et al. Gene-microarray analysis of multiple sclerosis lesions yields new targets validated in autoimmune encephalomyelitis. Nat. Med. 8, 500-508 (2002).

51. Werring, D. J. et al. The pathogenesis of lesions and normal-appearing white matter changes in multiple sclerosis: a serial diffusion MRI study. Brain 123, 1667-1676 (2000).

52. Morra, V. B. et al. Interferon-beta treatment decreases cholesterol plasma levels in multiple sclerosis patients. Neurology 62, 829-830 (2004).

53. Zhou, Y. X., Pannu, R., Le, T. Q. \& Armstrong, R. C. Fibroblast growth factor 1 (FGFR1) modulation regulates repair capacity of oligodendrocyte progenitor cells following chronic demyelination. Neurobiol. Dis. 45, 196-205 (2012).

54. Hibbits, N., Yoshino, J., Le, T. Q. \& Armstrong, R. C. Astrogliosis during acute and chronic cuprizone demyelination and implications for remyelination. ASN Neuro 4, 393-408 (2012).

55. Olah, M. et al. Identification of a microglia phenotype supportive of remyelination. Glia 60, 306-321 (2012).

56. Mohan, H. et al. Transcript profiling of different types of multiple sclerosis lesions yields FGF1 as a promoter of remyelination. Acta Neuropathol. Commun. 2, 168 (2014)

57. Furusho, M., Roulois, A. J., Franklin, R. J. \& Bansal, R. Fibroblast growth factor signaling in oligodendrocyte-lineage cells facilitates recovery of chronically demyelinated lesions but is redundant in acute lesions. Glia 63, 1714-1728 (2015).

58. Ornitz, D. M. \& Itoh, N. The fibroblast growth factor signaling pathway. Wiley Interdiscip. Rev. Dev. Biol. 4, 215-266 (2015).

59. Clemente, D., Ortega, M. C., Arenzana, F. J. \& de, C. F. FGF-2 and Anosmin-1 are selectively expressed in different types of multiple sclerosis lesions. J. Neurosci. 31, 14899-14909 (2011).

60. Han, M. H. et al. Proteomic analysis of active multiple sclerosis lesions reveals therapeutic targets. Nature 451, 1076-1081 (2008).

61. Ito, J., Nagayasu, Y., Miura, Y., Yokoyama, S. \& Michikawa, M. Astrocytes endogenous apoE generates HDL-like lipoproteins using previously synthesized cholesterol through interaction with ABCA1. Brain Res. 1570, 1-12 (2014).

62. Sheng, R. et al. Cholesterol selectively activates canonical Wnt signalling over non-canonical Wnt signalling. Nat. Commun. 5, 4393 (2014).

63. Kang, Z. et al. IL-17-induced Act1-mediated signaling is critical for cuprizoneinduced demyelination. J. Neurosci. 32, 8284-8292 (2012).

64. Winer, S. et al. Obesity predisposes to Th17 bias. Eur. J. Immunol. 39, 2629-2635 (2009).

65. Timmermans, S. et al. High fat diet exacerbates neuroinflammation in an animal model of multiple sclerosis by activation of the Renin Angiotensin system. J. Neuroimmune Pharmacol. 9, 209-217 (2014).

66. Haghikia, A. et al. Dietary fatty acids directly impact central nervous system autoimmunity via the small intestine. Immunity 43, 817-829 (2015).

67. Stromnes, I. M. \& Goverman, J. M. Active induction of experimental allergic encephalomyelitis. Nat. Protoc. 1, 1810-1819 (2006).

68. Brinkmann, B. G. et al. Neuregulin-1/ErbB signaling serves distinct functions in myelination of the peripheral and central nervous system. Neuron 59, 581-595 (2008). 
69. Steelman, A. J., Thompson, J. P. \& Li, J. Demyelination and remyelination in anatomically distinct regions of the corpus callosum following cuprizone intoxication. Neurosci. Res. 72, 32-42 (2012).

70. Thomson, C. E. et al. Myelinated, synapsing cultures of murine spina cord--validation as an in vitro model of the central nervous system. Eur. J. Neurosci. 28, 1518-1535 (2008).

\section{Acknowledgements}

We are grateful to Klaus-Armin Nave for constant support. We cordially thank Annett Fahrenholz, Tanja Freerck, Boguslawa Sadowski and Lennart Wiegand for technical support. We thank Prof Charles Stiles, Dr John Alberta and Prof Said Ghandour for generous gifts of antibodies. This work was funded by the Deutsche For-

schungsgemeinschaft (SA 2014/2-1 to G.S.). J.E. and W.M. were funded by an ERC Advanced grant awarded to Klaus-Armin Nave.

\section{Author contribution}

G.S., S.A.B., J.M.E., D.L. and W.M. participated in the planning and designing the experiments. S.A.B. was involved in performing all experiments. N.G. and J.M.E. performed cell culture experiments, S.K.S., P.D. and J.W., characterized the mouse pathologies, C.B. performed expression analyses, B.B. was involved in EAE experiments, L.H. and F.O. did flow cytometry with subsequent expression analyses, T.R. performed electron microscopy analysis, W.M. edited the manuscript, S.A.B., J.M.E. and G.S. wrote the manuscript

\section{Additional information}

Supplementary Information accompanies this paper at http://www.nature.com/ naturecommunications

Competing financial interests: The authors declare no competing financial interests. Reprints and permission information is available online at http://npg.nature.com/ reprintsandpermissions/

How to cite this article: Berghoff, S. A. et al. Dietary cholesterol promotes repai of demyelinated lesions in the adult brain. Nat. Commun. 8, 14241 doi: $10.1038 /$ ncomms14241 (2017).

Publisher's note: Springer Nature remains neutral with regard to jurisdictional claims in published maps and institutional affiliations.

(c) (f) This work is licensed under a Creative Commons Attribution 4.0 (c) (i) International License. The images or other third party material in this article are included in the article's Creative Commons license, unless indicated otherwise in the credit line; if the material is not included under the Creative Commons license, users will need to obtain permission from the license holder to reproduce the material. To view a copy of this license, visit http://creativecommons.org/licenses/by/4.0/

C The Author(s) 2017 
a

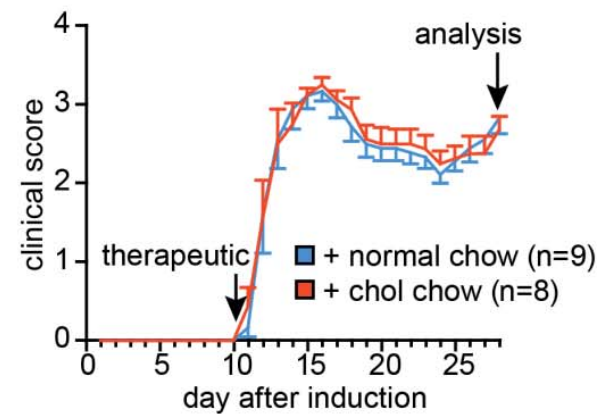

b
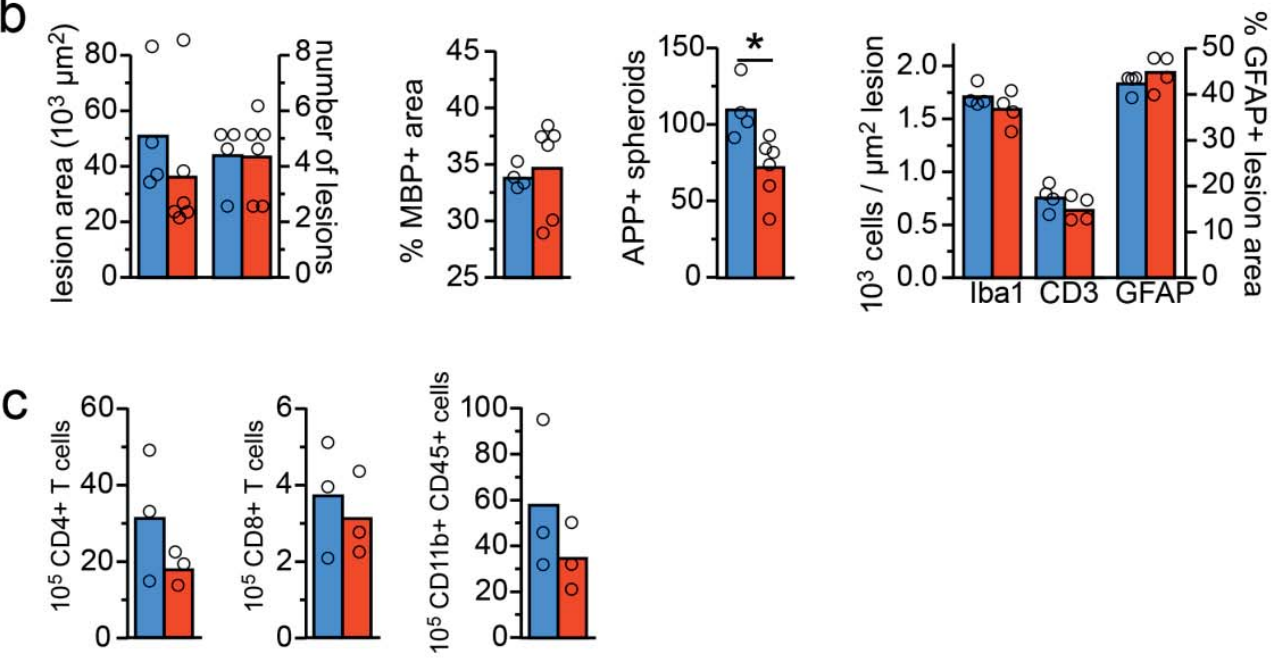

\section{Supplementary Figure 1.}

Therapeutically fed cholesterol does not aggravate EAE lesions.

a) Clinical score of mice with EAE, when cholesterol feeding started therapeutically with the onset of symptoms ( $n=8-9$ mice).

b) Histopathological assessment of lumbar spinal cord sections as done in prophylactic cholesterol feeding experiments (Fig. 1). LFB/PAS staining was used to determine the lesion area and number of lesions per section. Immuno-labeling for myelin basic protein was used to determine the percent of myelinated area within a lesion. On sections immuno-labeled for APP, the number of axonal speroids per square $\mathrm{mm}$ white matter area was counted, as a readout of axonal damage. Unpaired student's t-test revealed significantly less axonal damage in cholesterol fed animals $\left({ }^{*}, \mathrm{P}<0.05\right)$. Sections triple stained for microglia / macrophages, T cells, and astrocytes (Iba1-CD3-GFAP triple immuno-labeling) were used to assess the cellular composition of lesions. Bars represent mean values ( $n=4-6$ animals) with individual data points.

c) Absolute number of abTCR/CD4 and abTCR/CD8 T cells and CD45/CD11b myeloid (macrophages/microglia) cells per gram spinal cord was determined by flow cytometry from mice fed normal chow or cholesterol enriched chow ( $n=3$ animals). Bars represent means with individual data points. 


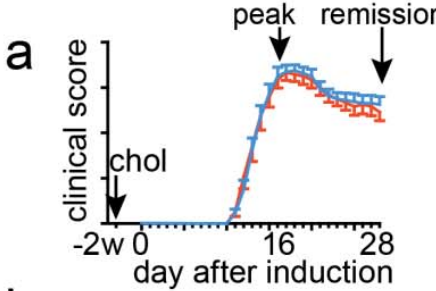

$\mathrm{b}$

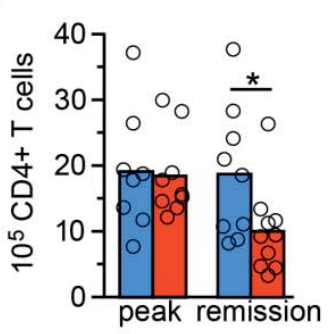

$\square+$ normal chow

$\square+$ chol chow

C
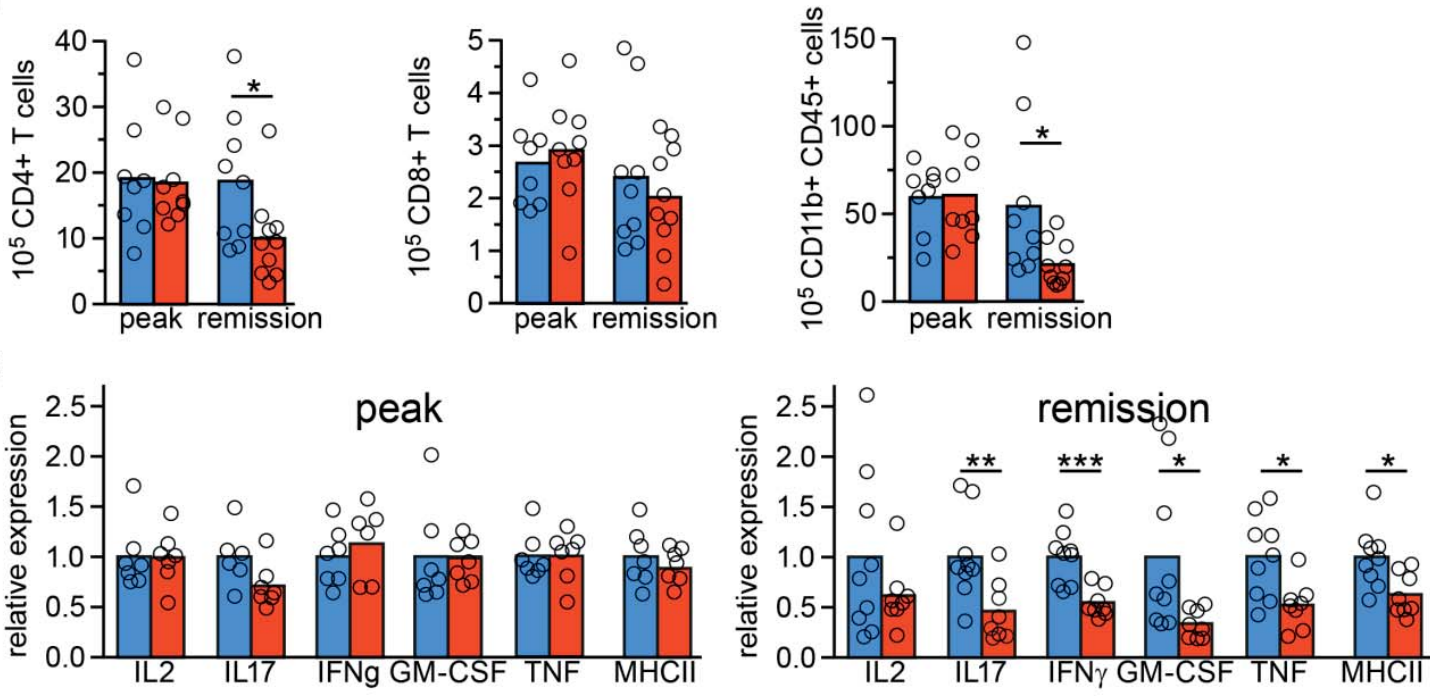

Supplementary Figure 2.

Immune cell infiltration and the inflammatory milieu in EAE.

a) Illustration of time points of analysis at the peak of clinical symptoms (16-18 dpi) and at remission (28 dpi), showing the clinical score of mice with EAE fed normal chow (bue) or cholesterol enriched chow (red).

b) Absolute number of abTCR/CD4 and abTCR/CD8 T cells and CD45/CD11b myeloid (macrophages/microglia) cells per gram spinal cord was determined by flow cytometry from mice fed normal chow or cholesterol enriched chow $(n=8-10$ animals, 2 independent experiments). Bars represent mean values with individual data points. One-way ANOVA with Tukey's post test revealed significantly less infiltration by CD4 T cells and reduced density of myeloid cells (microglia / macrophages) during remission ( ${ }^{*}, \mathrm{P}<0.05$ ).

c) Quantitative RT-PCR analysis to assess the inflammatory milieu in spinal cord lysates of mice fed normal chow or chow supplemented with cholesterol at peak of clinical symptoms and in remission, determining the expression of IL2, IL17, IFNg, TNF, GM-CSF, and MHC-II. Values were normalized to chow fed mice. Bars represent the means $(n=7$ animals at peak, $n=9$ animals in remission) with individual data points. Significance of cholesterol supplementation on expression of individual genes was assessed by student's tests (*, $\mathrm{P}<0.05 ;{ }^{* *}, \mathrm{P}<0.01$; $\left.{ }^{* *}, \mathrm{P}<0.001\right)$. 


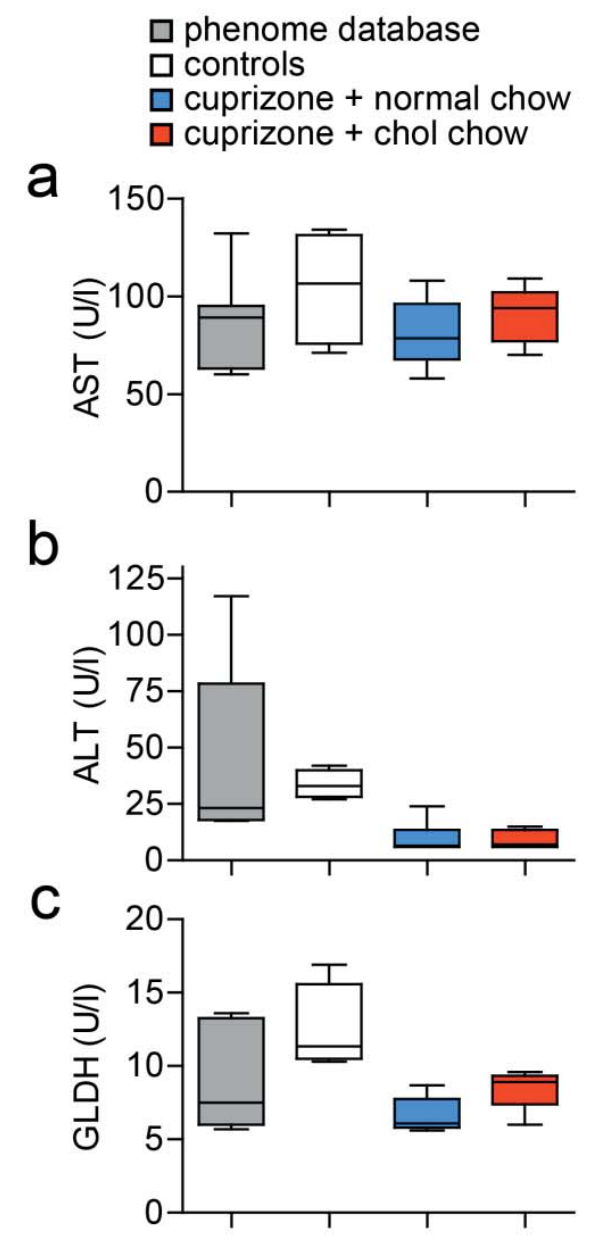

\section{Supplementary Figure 3.}

\section{Normal liver function in cuprizone treated animals.}

Activity of a) AST (aspartate transaminase), b) ALT (alanine transaminase), and c) GLDH (glutamate dehydrogenase) were determined in serum from mice after 6 weeks cuprizone with or without cholesterol supplementation ( $n=5-6$ animals) and own untreated controls $(n=4)$. Median with 2.5th - 97.5th percentiles were plotted together with published reference intervals (AST $n=7, A L T n=5$, GLDH $n=5$; Jackson Laboratory mouse phenome database (Supplementary Refs. 1, 2)). 
a

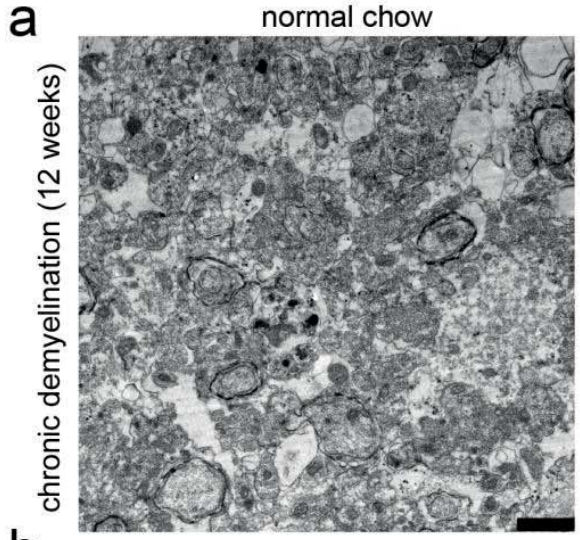

b
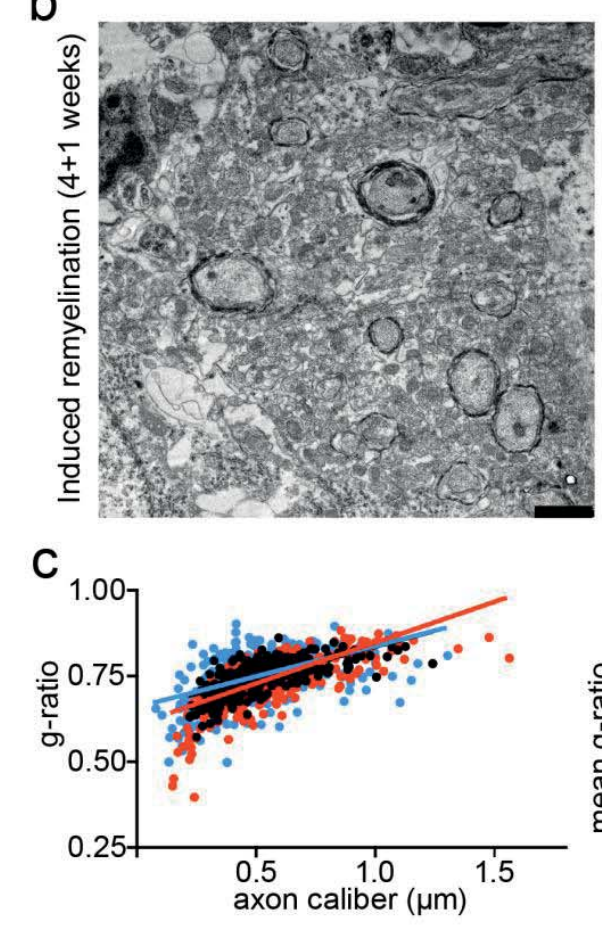

chol chow
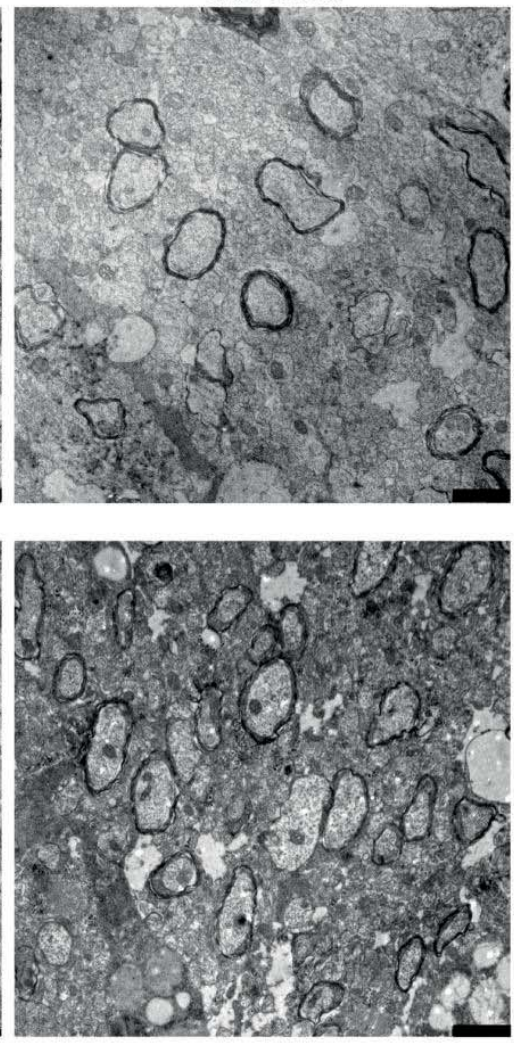

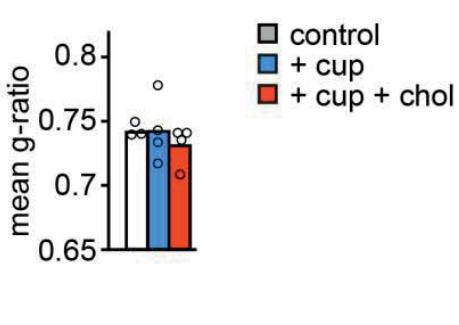

\section{Supplementary Figure 4.}

\section{Ultrastructural analysis of cuprizone treated mice.}

Representative electron micrographs of the corpus callosum of mice (a) that were chronically treated with cuprizone (12 weeks) with or without cholesterol chow or (b) were treated according to the "induced remyelination" paradigm (demyelination with cuprizone in normal chow for four weeks, followed by remyelination for 1 week with or without cholesterol chow, 4+1). Scale bars, $1 \mu \mathrm{m}$. c) G-ratio analysis and mean $g$ ratio in the corpus callosum of mice treated according to the „induced remyelination" paradigm $(4+1)$ showing individual data points and mean g-ratios of cuprizone treated mice with and without cholesterol supplementation ( $n=4$ animals) and untreated control mice ( $n=3$ animals; $80-100$ myelinated axons per animal). 


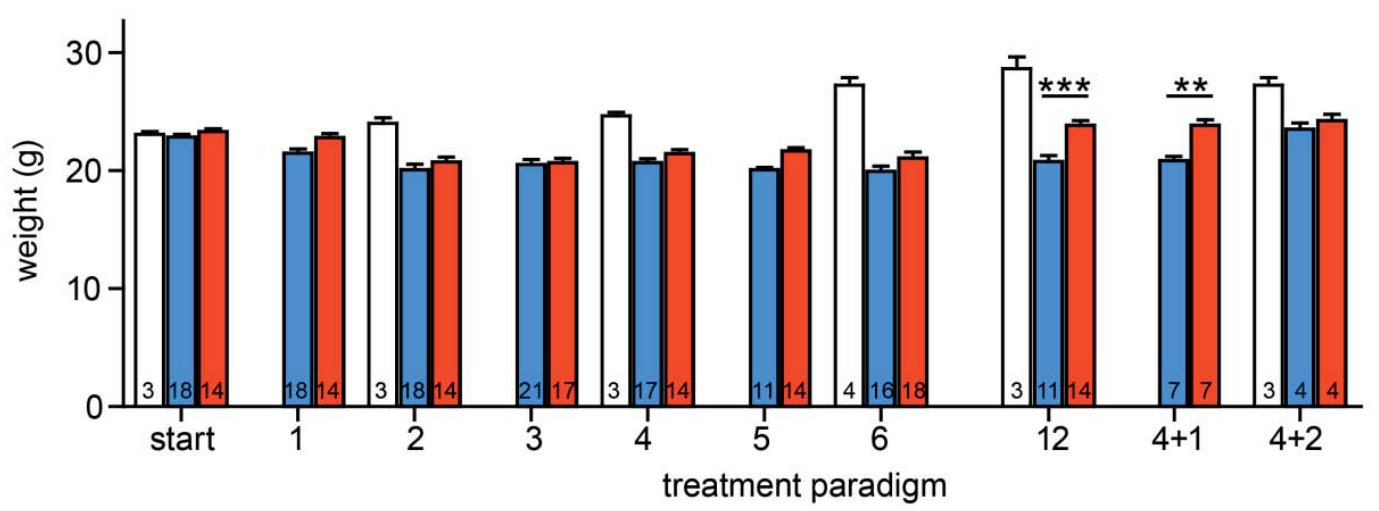

$$
\begin{aligned}
& \square \text { untreated control } \\
& \square+\text { normal chow } \\
& \square+\text { chol chow }
\end{aligned}
$$

\section{Supplementary Figure 5.}

Body weight of cuprizone treated animals.

Body weight of cuprizone treated animals on normal or cholesterol chow, and untreated control mice. Shown are mice at the beginning and from 1 to 12 weeks on cuprizone, and animals treated according to the "induced remyelination" paradigm (4+1 and 4+2, compare Fig. 5a). Number of animals at condition are depicted in the bars. Body weight is expressed as mean \pm s.e.m. Two way ANOVA revealed a significant influence of cholesterol on the weight of cuprizone treated animals $(P<0.0001)$, and Bonferoni post tests showed a significant difference at the 12 weeks $(P<0.0001)$ and $4+1(P<0.001)$ weeks time points. 


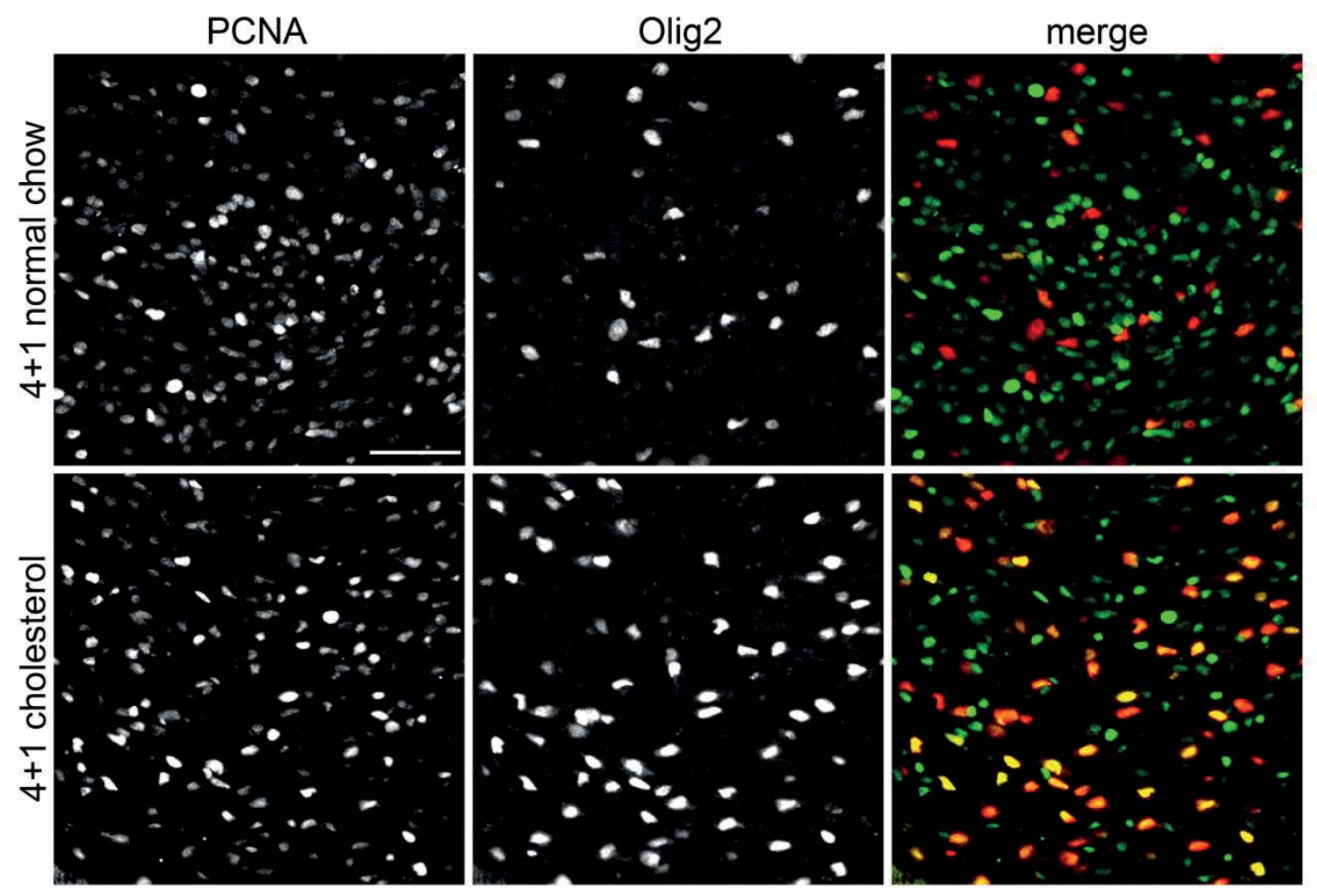

\section{Supplementary Figure 6.} OPC proliferation.

Representative images of proliferating OPCs (PCNA positive, Olig2 positive) in the corpus callosum of mice in the "induced remyelination" paradigm (demyelination for 4 weeks, followed by remyelination for one week, $4+1$ ) in the presence of absence of cholesterol supplementation (scale $50 \mu \mathrm{m}$ ). 

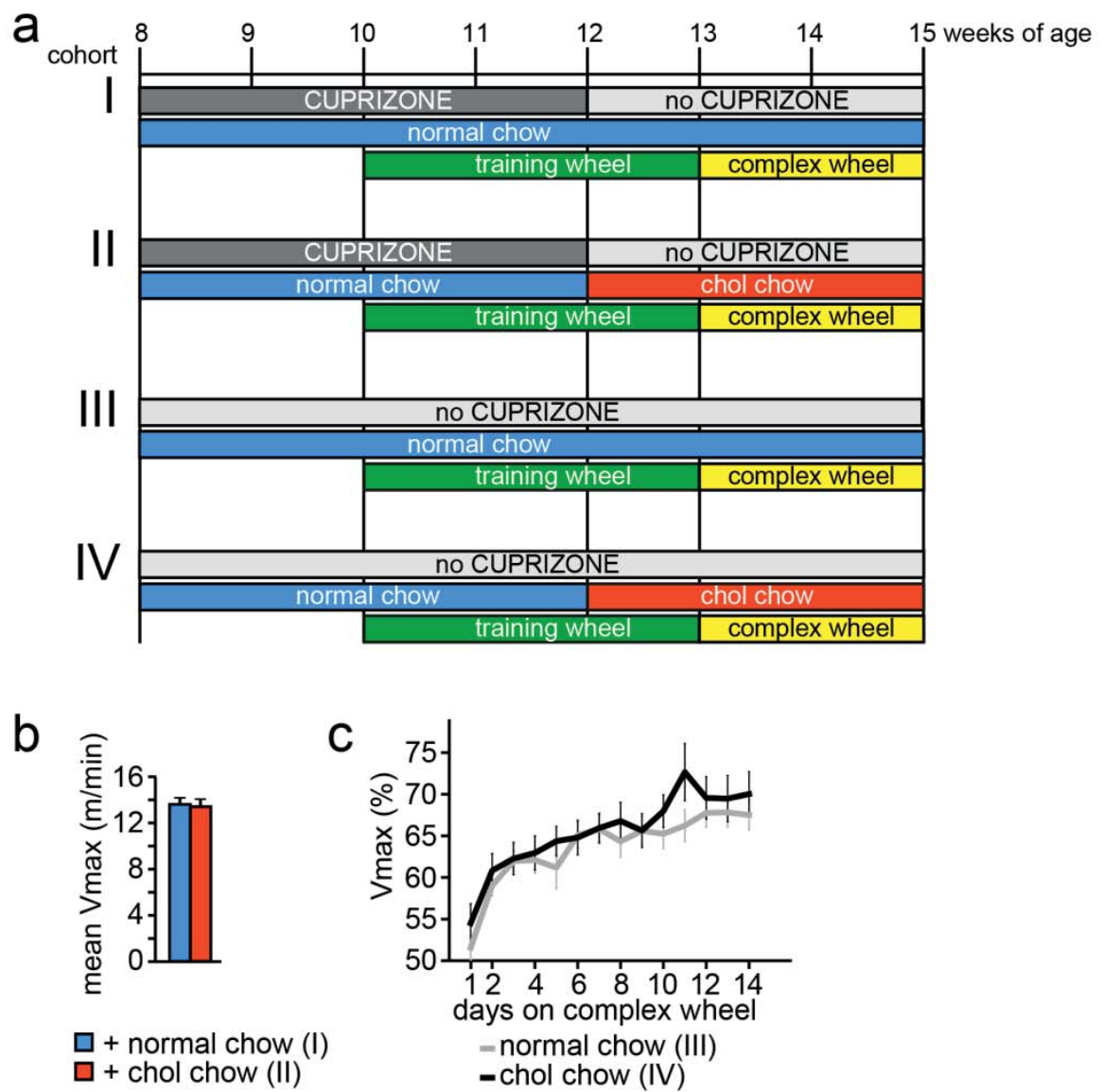

\section{Supplementary Figure 7.}

Motor performance on running wheel.

a) Treatment scheme of experimental cohorts. Mice in the "induced remyelination" paradigm (I, II) received cuprizone in normal chow for 4 weeks followed by normal chow with or without added cholesterol. Control groups (III, IV) did not receive cuprizone. A training wheel was placed into cages two weeks after the start of experiment for a duration of three weeks to improve cardiopulmonary and musculoskeletal strength. Mean maximum running speed (Vmax) of the last 7 days on the training wheel was set as reference $(100 \%)$ for each individual animal. Then a complex wheel replaced the training wheel and percent Vmax for each individual was calculated (control groups III and IV; experimental groups see Fig. $5 \mathrm{~g}$ ) which is a measure for bilateral sensorimotor coordination that likely involves the cerebellum and motor cortex and connecting white matter such as the corpus callosum.

b) Vmax on training wheel is not influenced by dietary cholesterol supplementation. Mean Vmax of each day of the third week on training wheel of cohorts I and II ( $n=6$ animals).

c) Vmax on complex wheel is not influenced by dietary cholesterol in control groups (III, IV) without cuprizone ( $n=13$ animals). 
a
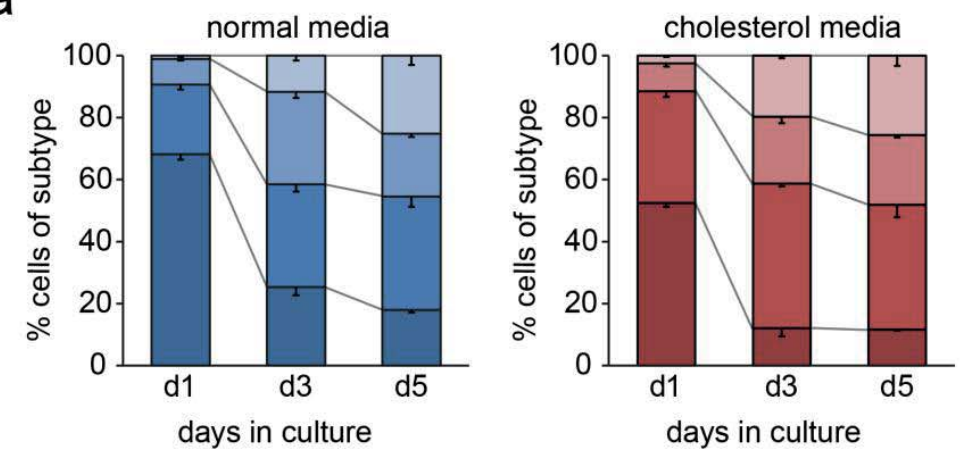

$\square \square$ mature OL

$\square \mathrm{MBP}+\mathrm{OL}$

an new OL

प्OPC

b
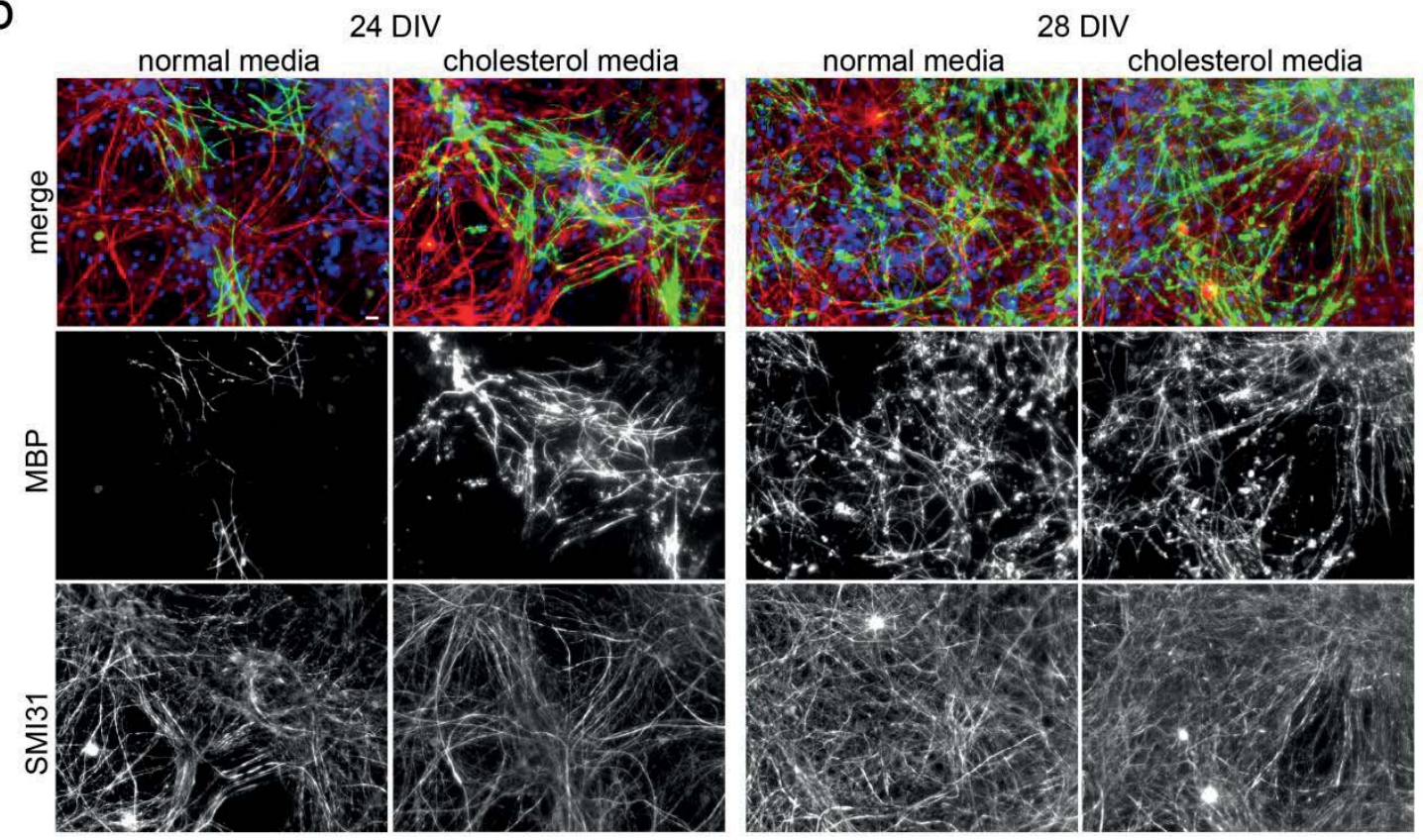

\section{Supplementary Figure 8.}

Increased the rate of oligodendrocyte differentiation and myelination in vitro.

a) Primary OPCs were plated in differentiating Sato media with or without $10 \mu \mathrm{g} / \mathrm{ml}$ cholesterol (f.c.). Coverslips were fixed after 1,3 or 5 days, stained for CNP and MBP and scored as outlined in the methods section.

b) Representative images of myelinating cocultures at 24 and 28 days in vitro (DIV), established from embryonic spinal cord in the presence or absence of cholesterol supplementation and stained for myelin segments (MBP, green), axons (SMI31, red), and nuclei (DAPI, blue) (scale bar, $20 \mu \mathrm{m}$ ). 


\section{Supplemental Table 1: Gene expression profile in corpus callosum of mice in "induced remyelination" paradigm}

Quantitative RT-PCR on dissected corpus callosi from mice after one week of induced remyelination with or without cholesterol (cuprizone for 4 weeks followed by one week remyelination) and untreated controls. Values are expressed as mean ratio \pm s.e.m. of untreated control mice (set to 1). Significance of cholesterol supplementation in cuprizone treated animals was assessed by student's $t$ tests ( $n=4$ animals per group).

\begin{tabular}{|c|c|c|c|c|}
\hline \multirow{2}{*}{$\begin{array}{l}\text { Induced remyelination } \\
\text { Gene name and function }\end{array}$} & \multirow[b]{2}{*}{ Gene symbol } & \multicolumn{2}{|c|}{ Fold expression } & \multirow[b]{2}{*}{$\mathrm{P}$ value } \\
\hline & & Normal diet & Chol diet & \\
\hline \multicolumn{5}{|l|}{ Oligodendrocyte genes } \\
\hline Oligodendrocyte lineage transcription factor 2 & Olig2 & $0.62 \pm 0.22$ & $2.19 \pm 0.13$ & 0.00087 \\
\hline Proteolipid protein 1 & Plp1 & $0.44 \pm 0,05$ & $2.38 \pm 0.17$ & 0.00015 \\
\hline Carbonic anhydrase 2 & Car2 & $0.20 \pm 0.18$ & $0.40 \pm 0.14$ & 0.00888 \\
\hline \multicolumn{5}{|l|}{ Glial markers } \\
\hline Allograft inflammatory factor 1 & Aif1 & $12.46 \pm 0.18$ & $4.86 \pm 0.24$ & 0.00772 \\
\hline Glial fibrillary acidic protein & Gfap & $12.29 \pm 0.07$ & $15.42 \pm 0.05$ & 0.01873 \\
\hline S100 calcium-binding protein beta & $S 100 b$ & $1.53 \pm 0.18$ & $2.22 \pm 0.05$ & 0.04870 \\
\hline \multicolumn{5}{|l|}{ Cholesterol synthesis and regulation } \\
\hline 3-Hydroxy-3-methylgutaryl-CoA synthase 1 & Hmgcs1 & $0.06 \pm 0.23$ & $0.20 \pm 0.29$ & 0.00817 \\
\hline 3-Hydroxy-3-methylgutaryl-CoA reductase 1 & $\mathrm{Hmgcr}$ & $0.19 \pm 0.19$ & $0.38 \pm 0.09$ & 0.00715 \\
\hline Mevalonate kinase & Mvk & $0.41 \pm 0.13$ & $0.69 \pm 0.06$ & 0.00368 \\
\hline Farnesyl-diphosphate farnesyltransferase 1 & Fdft1 & $0.39 \pm 0.03$ & $0.67 \pm 0.02$ & 0.00087 \\
\hline Sterol 14 alpha-demethylase & Cyp51a1 & $0.19 \pm 0.06$ & $0.27 \pm 0.08$ & 0.00465 \\
\hline 24-Dehydrocholesterol reductase & Dhcr24 & $0.19 \pm 0.35$ & $0.60 \pm 0.29$ & 0.02004 \\
\hline Sterol regulatory element binding transcription factor 2 & Srebf2 & $0.37 \pm 0.14$ & $0.51 \pm 0.06$ & 0.04666 \\
\hline \multicolumn{5}{|l|}{ Cholesterol uptake, transport and secretion } \\
\hline Apolipoprotein E & Apoe & $8.73 \pm 0.09$ & $7.94 \pm 0.09$ & 0.37224 \\
\hline Low density lipoprotein receptor-related protein 1 & Lrp1 & $3.56 \pm 0.06$ & $6.41 \pm 0.12$ & 0.00167 \\
\hline Low density lipoprotein receptor & Ldlr & $0.31 \pm 0.08$ & $0.79 \pm 0.14$ & 0.00040 \\
\hline Very low density lipoprotein receptor & VIdlr & $0.58 \pm 0.09$ & $0.87 \pm 0.16$ & 0.03860 \\
\hline ATP-binding cassette transporter A1 & Abca1 & $29.41 \pm 0.14$ & $11.22 \pm 0.03$ & 0.00015 \\
\hline Sterol 27-hydroxylase & Cyp27a1 & $1.84 \pm 0.36$ & $2.02 \pm 0.32$ & 0.81516 \\
\hline Cholesterol 25-Hydroxylase & Ch25h & $6.20 \pm 0.22$ & $7.20 \pm 0.37$ & 0.67972 \\
\hline Cholesterol 24-hydroxylase & Cyp46a1 & $0.16 \pm 0.35$ & $0.23 \pm 0.27$ & 0.30948 \\
\hline Liver $\mathrm{X}$ receptor alpha & Nr1h3 & $1.00 \pm 0.04$ & $0.96 \pm 0.10$ & 0.76459 \\
\hline Liver $\mathrm{X}$ receptor beta & Nr1h2 & $1.11 \pm 0.10$ & $0.99 \pm 0.03$ & 0.37539 \\
\hline Retinoic X receptor gamma & $R \times r g$ & $0.14 \pm 0.03$ & $0.15 \pm 0.09$ & 0.95361 \\
\hline \multicolumn{5}{|l|}{ Fibroblast growth factor family } \\
\hline Fibroblast growth factor 1 & Fgf1 & $0.44 \pm 0.16$ & $0.92 \pm 0.13$ & 0.00427 \\
\hline Fibroblast growth factor 2 & Fgf2 & $7.67 \pm 0.09$ & $3.12 \pm 0.11$ & 0.00021 \\
\hline Fibroblast growth factor 3 & Fgf3 & $0.03 \pm 0.79$ & $0.67 \pm 0.54$ & 0.00695 \\
\hline Fibroblast growth factor 7 & Fgf7 & $9.96 \pm 0.12$ & $5.23 \pm 0.15$ & 0.00589 \\
\hline Fibroblast growth factor 8 & Fgf8 & $0.95 \pm 0.17$ & $3.17 \pm 0.38$ & 0.00965 \\
\hline Fibroblast growth factor 9 & Fgf9 & $0.14 \pm 0.17$ & $0.44 \pm 0.28$ & 0.00447 \\
\hline Fibroblast growth factor 12 & Fgf12 & $0.20 \pm 0.21$ & $0.52 \pm 0.35$ & 0.02539 \\
\hline Fibroblast growth factor 17 & Fgf17 & $0.90 \pm .24$ & $1.79 \pm 0.13$ & 0.02323 \\
\hline Fibroblast growth factor 22 & Fgf22 & $1.44 \pm 0.27$ & $5.27 \pm 0.51$ & 0.03037 \\
\hline \multicolumn{5}{|l|}{ Other growth factors } \\
\hline Brain-derived neurotropic factor & $B d n f$ & $0.16 \pm 0.16$ & $0.19 \pm 0.28$ & 0.53091 \\
\hline Ciliary neurotrophic factor & Cntf & $20.77 \pm 0.24$ & $19.54 \pm 0.39$ & 0.87452 \\
\hline Epidermal growth factor & Egf & $7.11 \pm 0.16$ & $6.56 \pm 0.21$ & 0.71377 \\
\hline Insulin like growth factor 1 & $\lg 1$ & $11.72 \pm 0.11$ & $13.88 \pm 0.18$ & 0.35575 \\
\hline Nerve growth factor & Ngf & $2.00 \pm 0.23$ & $2.00 \pm 0.20$ & 0.94831 \\
\hline Neurotrophin 3 & Ntf3 & $1.35 \pm 0.21$ & $1.23 \pm 0.42$ & 0.82436 \\
\hline Platelet derived growth factor alpha & Pdgfa & $12.59 \pm 0.04$ & $8.44 \pm 0.02$ & 0.00003 \\
\hline Bone morphogenic protein 2 & Bmp2 & $1.35 \pm 0.21$ & $2.18 \pm 0.10$ & 0.04285 \\
\hline Bone morphogenic protein 4 & Bmp4 & $8.34 \pm 0.18$ & $7.55 \pm 0.20$ & 0.61971 \\
\hline Sonic hedgehog & Shh & $0.24 \pm 0.22$ & $0.39 \pm 0.08$ & 0.03463 \\
\hline Inhibin beta-A & Inhba & $2.49 \pm 0.14$ & $2.31 \pm 0.33$ & 0.80868 \\
\hline Pleiotrophin & Ptn & $4.44 \pm 0.19$ & $4.93 \pm 0.25$ & 0.64768 \\
\hline
\end{tabular}




\section{Supplemental Table 2: Gene expression profile in corpus callosum of mice in "chronic demyelination" paradigm}

Quantitative RT-PCR on dissected corpus callosi from chronically demyelinated mice with or without cholesterol (cuprizone for 12 weeks) and untreated controls. Values are expressed as mean ratio \pm s.e.m. of untreated control mice (set to 1 ). Significance of cholesterol supplementation in cuprizone treated animals was assessed by student's tests $(n=4$ animals per group).

\begin{tabular}{|c|c|c|c|c|}
\hline \multirow{2}{*}{$\begin{array}{c}\text { Chronic cuprizone } \\
\text { Gene name and function }\end{array}$} & \multirow[b]{2}{*}{ Gene symbol } & \multicolumn{2}{|c|}{ Fold expression } & \multirow[b]{2}{*}{ P value } \\
\hline & & Normal diet & Chol diet & \\
\hline \multicolumn{5}{|l|}{ Oligodendrocyte genes } \\
\hline Oligodendrocyte lineage transcription factor 2 & Olig2 & $0.24 \pm 0.06$ & $0.38 \pm 0.03$ & 0.00000 \\
\hline Proteolipid protein 1 & Plp1 & $0.10 \pm 0.10$ & $0.19 \pm 0.11$ & 0.00120 \\
\hline Carbonic anhydrase 2 & Car2 & $0.16 \pm 0.03$ & $0.32 \pm 0.14$ & 0.00417 \\
\hline \multicolumn{5}{|l|}{ Glial markers } \\
\hline Allograft inflammatory factor 1 & Aif1 & $2.49 \pm 0.18$ & $2.24 \pm 0.28$ & 0.45346 \\
\hline Glial fibrillary acidic protein & Gfap & $6.67 \pm 0.08$ & $4.75 \pm 0.04$ & 0.00104 \\
\hline S100 calcium-binding protein beta & $S 100 b$ & $1.96 \pm 0.05$ & $1.47 \pm 0.11$ & 0.00870 \\
\hline \multicolumn{5}{|l|}{ Cholesterol synthesis and regulation } \\
\hline 3-Hydroxy-3-methylgutaryl-CoA synthase 1 & Hmgcs1 & $0.32 \pm 0.13$ & $0.33 \pm 0.03$ & 0.84110 \\
\hline 3-Hydroxy-3-methylgutaryl-CoA reductase1 & $\mathrm{Hmgcr}$ & $0.73 \pm 0.02$ & $0.59 \pm 0.08$ & 0.00404 \\
\hline Mevalonate kinase & Mvk & $0.96 \pm 0.02$ & $0.70 \pm 0.12$ & 0.00281 \\
\hline Farnesyl-diphosphate farnesyltransferase 1 & Fdft1 & $0.87 \pm 0.02$ & $0.72 \pm 0.05$ & 0.00870 \\
\hline Sterol 14 alpha-demethylase & Cyp51a1 & $0.42 \pm 0.20$ & $0.48 \pm 0.07$ & 0.53237 \\
\hline 24-Dehydrocholesterol reductase & Dhcr24 & $0.51 \pm 0.16$ & $0.57 \pm 0.06$ & 0.55446 \\
\hline Sterol regulatory element binding transcription factor 2 & Srebf2 & $0.88 \pm 0.06$ & $0.72 \pm 0.04$ & 0.00700 \\
\hline \multicolumn{5}{|l|}{ Cholesterol uptake, transport and secretion } \\
\hline Apolipoprotein E & Apoe & $2.04 \pm 0.05$ & $1.52 \pm 0.11$ & 0.00834 \\
\hline Low density lipoprotein receptor-related protein 1 & Lrp1 & $3.30 \pm 0.05$ & $2.25 \pm 0.11$ & 0.00133 \\
\hline Low density lipoprotein receptor & Ldlr & $0.83 \pm 0.03$ & $0.60 \pm 0.09$ & 0.00080 \\
\hline Very low density lipoprotein receptor & Vldlr & $2.14 \pm 0.12$ & $1.68 \pm 0.13$ & 0.39744 \\
\hline ATP-binding cassette transporter A1 & Abca1 & $5.17 \pm 0.09$ & $3.73 \pm 0.11$ & 0.00920 \\
\hline Sterol 27-hydroxylase & Cyp27a1 & $0.26 \pm 0.16$ & $0.25 \pm 0.03$ & 0.82481 \\
\hline Cholesterol 25-Hydroxylase & Ch25h & $1.57 \pm 0.22$ & $1.50 \pm 0.25$ & 0.85498 \\
\hline Cholesterol 24-hydroxylase & Cyp46a1 & $0.72 \pm 0.21$ & $0.64 \pm 0.09$ & 0.58260 \\
\hline Liver $\mathrm{X}$ receptor alpha & Nr1h3 & $0.51 \pm 0.02$ & $0.55 \pm 0.05$ & 0.59219 \\
\hline Liver $\mathrm{X}$ receptor beta & Nr1h2 & $0.98 \pm 0.07$ & $0.79 \pm 0.07$ & 0.12759 \\
\hline Retinoic X receptor gamma & Rxrg & $1.18 \pm 0.07$ & $1.08 \pm 0.13$ & 0.56939 \\
\hline \multicolumn{5}{|l|}{ Fibroblast growth factor family } \\
\hline Fibroblast growth factor 1 & Fgf1 & $0.54 \pm 0.01$ & $1.02 \pm 0.07$ & 0.00002 \\
\hline Fibroblast growth factor 2 & Fgf2 & $4.32 \pm 0.01$ & $2.70 \pm 0.06$ & 0.00057 \\
\hline Fibroblast growth factor 3 & Fgf3 & $0.79 \pm 0.10$ & $1.58 \pm 0.09$ & 0.00099 \\
\hline Fibroblast growth factor 7 & Fgf7 & $3.31 \pm 0.52$ & $3.76 \pm 0.52$ & 0.33069 \\
\hline Fibroblast growth factor 8 & Fgf8 & $0.39 \pm 0.27$ & $0.51 \pm 0.16$ & 0.31460 \\
\hline Fibroblast growth factor 9 & Fgf9 & $0.59 \pm 0.10$ & $0.60 \pm 0.06$ & 0.88087 \\
\hline Fibroblast growth factor 12 & Fgf12 & $0.47 \pm 0.08$ & $0.42 \pm 0.16$ & 0.51466 \\
\hline Fibroblast growth factor 17 & Fgf17 & $1.51 \pm 0.06$ & $2.30 \pm 0.02$ & 0.00000 \\
\hline Fibroblast growth factor 22 & Fgf22 & $3.57 \pm 0.15$ & $3.62 \pm 0.19$ & 0.94283 \\
\hline \multicolumn{5}{|l|}{ Other growth factors } \\
\hline Brain-derived neurotropic factor & $B d n f$ & $1.82 \pm 0.25$ & $2.45 \pm 0.65$ & 0.17036 \\
\hline Ciliary neurotrophic factor & Cntf & $5.32 \pm 0.09$ & $3.86 \pm 0.17$ & 0.02809 \\
\hline Epidermal growth factor & Egf & $1.93 \pm 0.14$ & $2.51 \pm 0.08$ & 0.02271 \\
\hline Insulin like growth factor 1 & $\operatorname{lgf1}$ & $2.79 \pm 0.03$ & $2.49 \pm 0.08$ & 0.01711 \\
\hline Nerve growth factor & $\mathrm{Ngf}$ & $0.39 \pm 0.09$ & $0.44 \pm 0.15$ & 0.48108 \\
\hline Neurotrophin 3 & Ntf3 & $0.61 \pm 0.17$ & $0.76 \pm 0.19$ & 0.20164 \\
\hline Platelet derived growth factor alpha & Pdgfa & $8.83 \pm 0.11$ & $4.70 \pm 0.19$ & 0.00305 \\
\hline Bone morphogenic protein 2 & Bmp2 & $1.01 \pm 0.25$ & $0.99 \pm 0.19$ & 0.92774 \\
\hline Bone morphogenic protein 4 & $B m p 4$ & $1.15 \pm 0.03$ & $1.73 \pm 0.20$ & 0.00429 \\
\hline Sonic hedgehog & Shh & $0.67 \pm 0.08$ & $0.58 \pm 0.27$ & 0.45346 \\
\hline Inhibin beta-A & Inhba & $1.33 \pm 0.11$ & $1.29 \pm 0.08$ & 0.78641 \\
\hline Pleiotrophin & Ptn & $1.89 \pm 0.08$ & $1.78 \pm 0.18$ & 0.68471 \\
\hline
\end{tabular}




\section{Supplementary Table 3: List of primer sequences}

All primers used for expression analysis were intron-spanning (5'-3'; forward - reverse).

\begin{tabular}{|c|c|}
\hline RplpO & GATGCCCAGGGAAGACAG - ACAATGAAGCATTTTGGATAATCA \\
\hline Hprt1 & TCCTCCTCAGACCGCTTTT - CCTGGTTCATCATCGCTAATC \\
\hline Plp1 & TCAGTCTATTGCCTTCCCTAGC - AGCATTCCATGGGAGAACAC \\
\hline Car2 & CAAGCACAACGGACCAGA - ATGAGCAGAGGCTGTAGG \\
\hline Olig2 & AGACCGAGCCAACACCAG - AAGCTCTCGAATGATCCTTCTTT \\
\hline Aif1 & TGTTTTTCTCCTCATACATCAGAATC - CCGAGGAGACGTTCAGCTAC \\
\hline Gfap & TGCTCCTGCTTCGAGTCCTT - CAAGAGGAACATCGTGGTAAAGA \\
\hline S100b & AACAACGAGCTCTCTCACTTCC - CTCCATCACTTTGTCCACCA \\
\hline Hmgcs1 & GGGTCTGATCCCCTTTGG - ACGATTCCCACATCTTTTGG \\
\hline $\mathrm{Hmgcr}$ & TGATTGGAGTTGGCACCAT - TGGCCAACACTGACATGC \\
\hline Mvk & CTCAAGGACGGGGTCTCC - GGCCCACTTGTTGATTGACT \\
\hline Fdft1 & TCAATCAGACCAGTCGCAGC - GTGCCGTATGTCCCCATCC \\
\hline Srebf2 & ACCTAGACCTCGCCAAAGGT - GCACGGATAAGCAGGTTTGT \\
\hline Apoe & GACCCTGGAGGCTAAGGACT - AGAGCCTTCATCTTCGCAAT \\
\hline Cyp51a1 & TTAGAACAGAAAGCAGTGTGTGG -TGCATCTATCAAGTAAATTCAGATCC \\
\hline Dhcr24 & GGTCATGACGGACGACGTA - AGGGCTTGTAGTAACTGCCAAT \\
\hline Lrp1 & ACCACCATCGTGGAAAATG - GTCCCAGCCACGGTGATA \\
\hline Ldlr & TTCCTGTCCATCTTCTTCCCTA - CAAAGTTTATGCTGTTGATTGTGA \\
\hline VIdlr & AAGTCAGTGTTCCCCCAAAA - TGCTGCCATCACTAAGAGCA \\
\hline Abca1 & CTGTTTCCCCCAACTTCTG - TCTGCTCCATCTCTGCTTTC \\
\hline Cyp27a1 & ATGGGATCTTCATCGCACA - CGTTTAAGGCATCCGTGTAGA \\
\hline Ch25h & TGCTACAACGGTTCGGAGC - AGAAGCCCACGTAAGTGATGAT \\
\hline Cyp46a1 & AACTTTGTCACCTTCTTCATTGC - CCATCACTGTGAATGCCAGA \\
\hline Fgf1 & GGACACCGAAGGGCTTTTAT - GCATGCTTCTTGGAGGTGTAA, \\
\hline Fgf2 & CCAACCGGTACCTTGCTATG - GATTCCAGTCGTTCAAAGAAGAA, \\
\hline Fgf3 & TGAGAACAGCGCCTATAGCA - GTACCGCCCAGAAAAGAGC, \\
\hline Fgf7 & GGCAATCAAAGGGGTGGA - CCTCCGCTGTGTGTCCATTTA, \\
\hline Fgf8 & GCTGAGCTGCCTGCTGTT - AGCTCGGAGCAGGGAAGT, \\
\hline Fgf9 & GGGGAGCTGTATGGATCAGA - TCCCGTCCTTATTTAATGCAA \\
\hline Fgf12 & CAAGGACGAAAACAGCGACT - CTCCATTCATGGCCACATAA \\
\hline Fgf17 & TATGAACAAGAGGGGCAAGC - CTCGGTGAACACGCAGTCT \\
\hline Fgf22 & CCAGGACAGTATAGTGGAGATCC - AGTAGACCCGCGACCCATAG \\
\hline Pdgfa & CTCTTGGAGATAGACTCCGTAG - ACTTCTCTTCCTGCGAATGG \\
\hline Cntf & GACCTGACTGCTCTTATGGAATCT - GCCTGGAGGTTCTCTTGGA \\
\hline lgf1 & AGCAGCCTTCCAACTCAATTAT - GAAGACGACATGATGTGTATCTTTATC \\
\hline Bdnf & GCATCTGTTGGGGAGACAAG - TGGTCATCACTCTTCTCACCTG \\
\hline Egf & TTGTGACACCTGGAAAACTGA - GTTTGAGAAGTTCGGGGTCA \\
\hline Ngf & TATACTGGCCGCAGTGAGGT - GGACATTGCTATCTGTGTACGG \\
\hline Ntf3 & GCCAGGCCGGTCAAAAAC - GACAAGGCACACACACAG \\
\hline Bmp2 & CGGACTGCGGTCTCCTAA - GGGGAAGCAGCAACACTAGA \\
\hline Bmp4 & GAGGAGTTTCCATCACGAAGA - GCTCTGCCGAGGAGATCA \\
\hline Shh & TCCACTGTTCTGTGAAAGCAG - GGGACGTAAGTCCTTCACCA \\
\hline Inhba & GGGAGTGATCCCTGGAAAC - TCCTCTTCATGGTATTGGCACT \\
\hline Ptn & CCTCAAGCGGAGTCAAAGAA - CTTTTCCTGGTCCACAGACG \\
\hline Actb & GTACAACCTCCTTGCAGC TCCT - TTG TCG ACGACGAGCGC \\
\hline Actb probe & CGCCACCAGTTCGCCATGGAT \\
\hline Ifng & TCAAGTGGCATAGATGTGGAA GAA -TGGCTCTGCAGGATTTTC ATG \\
\hline Ifng probe & TCA CCATCCTTT TGC CAGTTCCTCCAG \\
\hline IL17 & ACT TTC AGG GTC GAG AAG ATG CT-TTC TGA ATC TGC CTC TGA ATC CAC \\
\hline IL17 probe & TGGGTGTGGGCTGCACCTGC \\
\hline 112 & CAGGATGCTCACCTTCAA ATT TT - CGCAGAGGTCCAAGTTCATCT \\
\hline II2 probe & CTTGCCCAAGCAGGCCACAGA ATTG \\
\hline Gmcsf & GGGCGCCTTGAACATGAC - CGCATAGGTGGTAAC TTGTGT TTC \\
\hline Gmcsf probe & CCCCCCAACTCCGGAAACGGA \\
\hline Tnf & TCGAGTGACAAGCCCGTAGC - CTCAGCCACTCCAGCTCC TC \\
\hline Tnf probe & CGTCGTAGCAAACCACCAAGC AGA \\
\hline H2-DMb2 (MHCII) & AGCCTTATTCATCGCTTG CAA - AGATGGCGCTCTCGTTCTGT \\
\hline H2-DMb2probe & ACGGGCTTCAGG ACTGTGCCA CC \\
\hline
\end{tabular}


Supplementary Table 4: Microscope settings

In all recorded images, LUT was linear covering the full range of data. All experiments were done at $20^{\circ} \mathrm{C}$, the depth of all pictures was 8 bit.

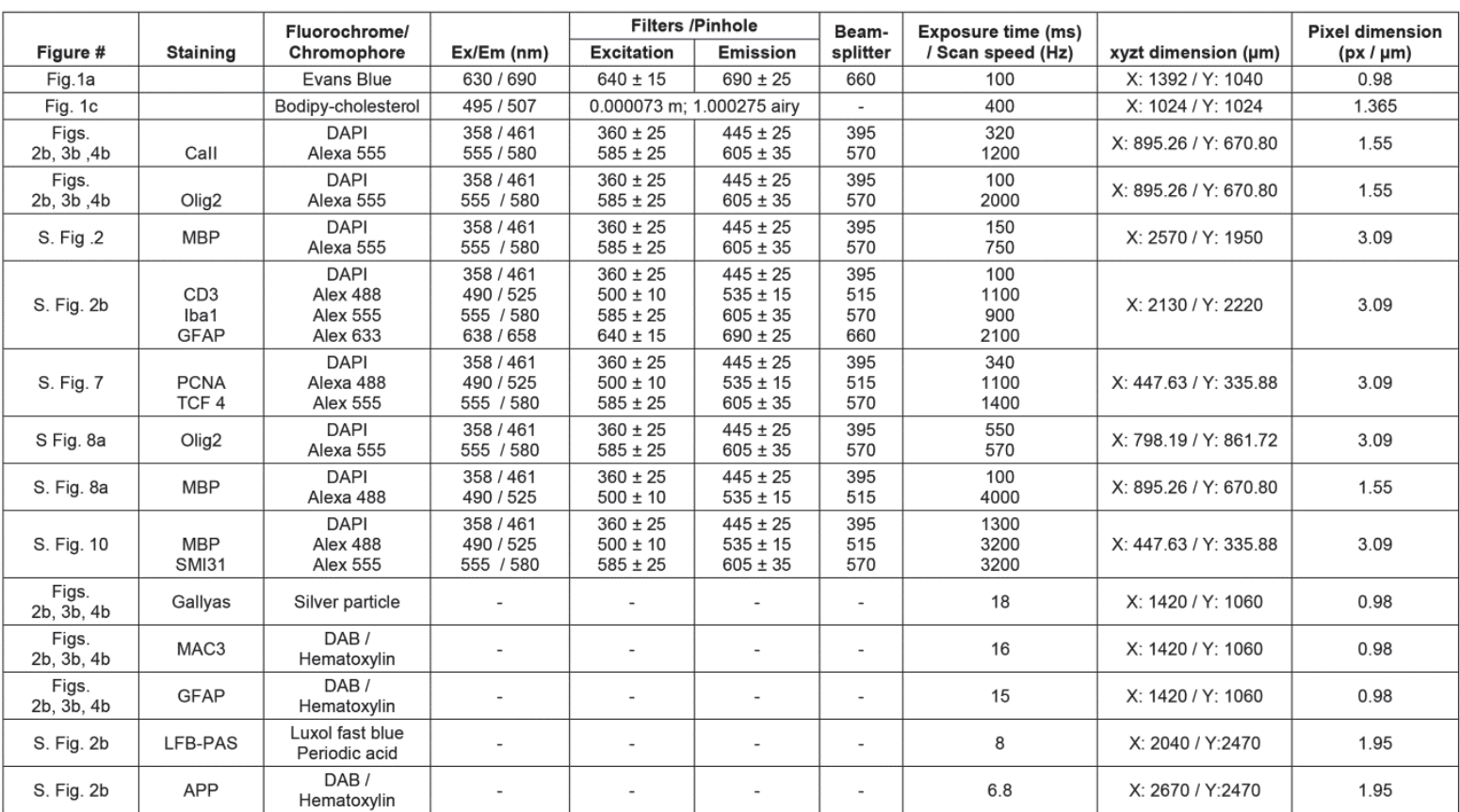

Supplementary References

1. Bradford, B.U., et al. Interstrain differences in the liver effects of trichloroethylene in a multistrain panel of inbred mice. Toxicol Sci 120, 206-217 (2011).

2. Shockley K, Paigen B, Churchill GA. Diet effects on blood chemistry and lipids in 10 inbred strains of mice. Mouse Phenome Database web site, The Jackson Laboratory, Bar Harbor, Maine USA. http://phenome.jax.org 


\subsection{Publication 2:}

Blood-brain barrier hyperpermeability precedes demyelination in the cuprizone model

Stefan A. Berghoff ${ }^{1}$, Tim Düking ${ }^{1}$, Lena Spieth ${ }^{1}$, Jan Winchenbach ${ }^{1}$, Sina K. Stumpf ${ }^{1}$, Nina Gerndt ${ }^{1}$, Kathrin Kusch ${ }^{1}$, Torben Ruhwedel ${ }^{1}$, Wiebke Möbius ${ }^{1,2}$ and Gesine Saher ${ }^{1}$

${ }^{1}$ Department of Neurogenetics, Max-Planck-Institute of Experimental Medicine, Hermann-Rein-Str. 3, 37075 Goettingen, Germany

${ }^{2}$ Center Nanoscale Microscopy and Molecular Physiology of the Brain (CNMPB), Wilhelmsplatz 1, 37073 Göttingen, Germany. 


\title{
Blood-brain barrier hyperpermeability precedes demyelination in the cuprizone model
}

Stefan A. Berghoff', Tim Düking', Lena Spieth', Jan Winchenbach', Sina K. Stumpf ${ }^{1}$, Nina Gerndt ${ }^{1}$, Kathrin Kusch', Torben Ruhwedel', Wiebke Möbius ${ }^{1,2}$ and Gesine Saher ${ }^{1 *}$ (D)

\begin{abstract}
In neuroinflammatory disorders such as multiple sclerosis, the physiological function of the blood-brain barrier (BBB) is perturbed, particularly in demyelinating lesions and supposedly secondary to acute demyelinating pathology. Using the toxic non-inflammatory cuprizone model of demyelination, we demonstrate, however, that the onset of persistent BBB impairment precedes demyelination. In addition to a direct effect of cuprizone on endothelial cells, a plethora of inflammatory mediators, which are mainly of astroglial origin during the initial disease phase, likely contribute to the destabilization of endothelial barrier function in vivo. Our study reveals that, at different time points of pathology and in different CNS regions, the level of gliosis correlates with the extent of BBB hyperpermeability and edema. Furthermore, in mutant mice with abolished type 3 CXC chemokine receptor (CXCR3) signaling, inflammatory responses are dampened and BBB dysfunction ameliorated. Together, these data have implications for understanding the role of BBB permeability in the pathogenesis of demyelinating disease.
\end{abstract}

Keywords: Blood-brain barrier, Demyelination, Gliosis, Astrocyte, Inflammatory mediators

\section{Introduction}

The cerebral vasculature controls and restricts the transport of biomolecules between blood and the CNS parenchyma by means of the blood-brain barrier (BBB) [1,37]. While specialized brain endothelial cells are physically connected via unique belt-like tight junctions that mediate BBB tightness, perivascular cells and astrocytes also contribute to BBB physiology, collectively forming the neurovascular unit (NVU). In a wide range of neurological disorders including multiple sclerosis (MS) increased vascular permeability has been observed [33] but the primary cause for the pathophysiology of the NVU and the relation to disease specific pathomechanisms remains unclear.

MS is an acquired inflammatory demyelinating disease of the CNS in which BBB permeability is increased in both newly forming demyelinating lesions and even in normal appearing white matter [18, 59]. BBB impairment is observed at the onset of clinical symptoms in

\footnotetext{
* Correspondence: saher@em.mpg.de

Department of Neurogenetics, Max-Planck-Institute of Experimenta Medicine, Hermann-Rein-Str. 3, 37075 Goettingen, Germany Full list of author information is available at the end of the article
}

experimental autoimmune encephalomyelitis (EAE), an animal model of MS, coinciding with initial immune cell infiltration and glial activation $[19,51]$. In this model, pro-inflammatory cytokines/chemokines produced by activated immune cells on the peripheral side of the barrier, or by glial cells in the CNS, contribute to $\mathrm{BBB}$ hyperpermeability $[4,42]$. Thus, BBB disruption could potentially be secondary to pathology. However, it is unclear whether demyelination or other disease factors cause BBB disturbances.

We previously showed in the non-inflammatory cuprizone model of demyelination [45], that BBB permeability is increased at the peak of demyelinating disease, and that this BBB dysfunction can be utilized for CNS delivery of therapeutics [11]. However, it is unclear which pathomechanism triggers the $\mathrm{BBB}$ breach in the cuprizone model. Here, we further characterize these BBB disturbances, relating BBB pathophysiology to histopathology in different brain regions and at different stages of disease progression. We demonstrate that early disease processes are associated with elevated levels of several pro-inflammatory mediators of predominantly astroglial 
origin. This local inflammatory milieu, together with a primary effect of cuprizone on endothelial cells, leads to the downregulation of BBB maintenance factors, endothelial efflux transporters, and tight junction proteins resulting in morphological disruption of tight junctions. These endothelial disturbances are associated with local hyperpermeability of the $\mathrm{BBB}$ and edema, even before the onset of demyelination.

\section{Materials and methods \\ Mice}

All animal studies were performed in compliance with the animal policies of the Max Planck Institute of Experimental Medicine, and were approved by the German Federal State of Lower Saxony. Adult male C57BL/6N mice or CX3CR1 ${ }^{\mathrm{GFP} / \mathrm{GFP}}$ mice [26] were taken at 810 weeks of age. Animals were randomly assigned to an experimental group. Cuprizone $(0.2 \% w / w$, Sigma $)$ was mixed into powder chow (V1124 ssniff).

\section{Histological analyses}

Histological analyses were done as described [11, 49] with minor modifications. Anesthetized mice were perfused with paraformaldehyde (PFA) and brains were cut by vibratome $(40 \mu \mathrm{m}$, Leica VT1200)) or embedded in paraffin. Brain sections (HMP 110, MICROM) at Bregma -1.58 were taken for histological characterization using standard protocols using LSAB2 (Dako) or Vector Elite ABC (Vector Labs). For occludin and ZO1 staining, animals were perfused with PFA containing $0.2 \%$ glutaraldehyde and cut on a vibratome. Tissue sections or fixed endothelial cells were processed for immunolabeling by permeabilization (0.4\% Triton X-100 in PBS), blocking (4\% horse serum, $2 \%$ BSA, $0.2 \%$ Triton X-100 in PBS) and incubation with first antibody (1\% HS, $0.05 \%$ Triton X-100 in PBS). Incubation with fluorophore coupled secondary antibodies (ThermoFisher) and DAPI (4',6-diamidino-2 phenylindole) were done in $1.5 \%$ HS in PBS, after which sections were mounted in AquaPolymount (Polysciences). Gallyas silver impregnation was done as described [49]. Specimens were analyzed on an Axio Imager.Z1 (Zeiss) equipped with an AxioCam MRc3, $\times 0.63$ Camera Adaptor and the ZEN 2012 blue edition software using $\times 10$ objective (Plan Apochromat $\times 10 / 0.45$ M27) or $\times 20$ objective (Plan-Apochromat $\times 20 / 0.8$ ) or by confocal laser scanning microscopy (Leica SP5 HCX PL APO CS 63×/1.20) using the Leica Confocal Software (Leica Microsystems). Quantification of positive areas in the corpus callosum above the fornix were done by semi-automated analysis with ImageJ software macro and color deconvolution plug-in. Vessel paint was performed as described [34] with minor modifications. Mice were intravenously injected with $200 \mu \mathrm{l}$ of $20 \mathrm{mg} / \mathrm{ml}$ FITC-Dextran (46,945, Sigma-Aldrich Inc., Germany). After $30 \mathrm{~min}$ circulation time animals were anaesthetized, flushed, stained with DID (ThermoFischer, D7757) and fixed with PFA before sectioning with a vibratome $(100 \mu \mathrm{m})$. All Images were processed with NIH ImageJ and Adobe Photoshop CS5.1 software. Electron microscopic analysis was done as previously described [49]. Briefly, tissue was fixed in 4\% PFA, $2.5 \%$ glutaraldehyde, $0.1 \mathrm{M}$ Phosphate buffer and sagittal sections were cut on a vibratome (Leica VT1200). The corpus callosum with adjacent tissue was punched and embedded in epon (LYNXII, EMS). Ultrathin uranyl acetate contrasted sections were imaged with a LEO EM912 AB (Zeiss) equipped with a $2 \mathrm{k}-\mathrm{CCD}$ camera (TRS, Moorenweis).

\section{Blood-brain barrier permeability}

Tracer injections were done as described $[11,49]$ with minor modifications. For measurement of BBB permeability, tracers were i.v. injected (Evans blue $50 \mathrm{mg} \mathrm{g}^{-1}$ body weight; sodium fluorescein $200 \mathrm{mg} \mathrm{g}^{-1}$ body weight). After $4 \mathrm{~h}$ incubation, animals were perfused with PBS to remove tracer from the circulatory system. The region of interest was dissected, and tissue samples were weighed. For lyophilization, tissues were exposed to a shelf temperature of $-56{ }^{\circ} \mathrm{C}$ for $24 \mathrm{~h}$ under vacuum of $0.2 \mathrm{mBar}$ (Christ LMC-1 BETA 1-16). Samples were weighed for calculation of water content and edema. Lyophilized tissue samples were extracted with formamide at $57{ }^{\circ} \mathrm{C}$ for $24 \mathrm{~h}$ on a shaker at $300 \mathrm{rpm}$ (Eppendorf Thermomixer). Integrated density of tracer fluorescence was determined in triplicates after 1:3 ethanol dilution to increase sensitivity. Tracer concentration was calculated using a standard curve of tracer spiked brain samples.

\section{Immunoblot}

Brain tissue samples were lysed in sucrose buffer (18\% sucrose, $10 \mathrm{mM}$ Tris/HCl pH 7.4, $1 \mathrm{mM}$ sodium hydrogen carbonate, $1 \mathrm{mM}$ magnesium chloride, $0.1 \%$ Triton, $0.2 \%$ lithiumdodecyl sulphate, $0.025 \%$ sodium deoxycholate) with protease inhibition (Roche) using a Precellys 24 homogenizer (Bertin technologies). Detection of immunolabeled proteins was performed with ECL detection reagent (Perkin Elmer) using ChemoCam Imager (Intas).

\section{Magnetic cell isolation}

Glial cells were isolated according to the adult brain dissociation protocol (Miltenyi biotec). Corpus callosum and cortex were isolated using a brain matrix from Bregma +1.10 to -2.46 . Antibody labeling steps were done according to the respective antibody Microbead kit protocol (Miltenyi biotec), oligodendrocytes (O4, 130-096-670); astrocytes (ACSA-2, 130-097-679), microglia (CD11b, 130093-636), and endothelial cells (CD31, 130-091-935). Purity of cell populations was routinely determined by qPCR on extracted and reverse transcribed RNA (see below) and revealed only minimal contamination by other cell types. 


\section{Cell cultures}

Primary mouse brain endothelial cell cultures were established from 7 days old mice or rats. Briefly, cortices were digested with $1 \mathrm{mg} / \mathrm{ml}$ collagenase/dispase and $2.5 \mu \mathrm{g} / \mathrm{ml}$ DNAse (Roche) in dissection buffer (HBSS, $10 \mathrm{mM}$ HEPES, $0.5 \%$ BSA, $5000 \mathrm{U} / \mathrm{ml}$ penicillin/streptomycin) for $45 \mathrm{~min}$ at $37^{\circ} \mathrm{C}$. After trituration, cells were resuspended in 25\% BSA and centrifuged at $1000 \mathrm{~g}$ for $20 \mathrm{~min}$ to pellet microvessels. Isolated microvessels from individual mice were plated in Endobasal Medium (Promocell) with $0.4 \%$ puromycin for positive selection on coverslips or polyester transwell inserts (Corning). Primary astrocyte cultures were prepared from 0 to 2 days old mice as previously described [11]. Primary microglia cultures were prepared from P0-P2 old mice by differential shaking of mixed glial cultures as described [55]. Cell purity was routinely determined by immune stainings and always exceeded 95\%. For coculture experiments, endothelial cells cultured in transwell inserts above astrocytes plated on the bottom of the well plate. Confluent cells cultures were treated with a final concentration of $250 \mu \mathrm{M}$ cuprizone in $0.125 \%$ DMSO or in $0.125 \%$ DMSO alone for up to $72 \mathrm{~h}$. An epithelial Voltohmmeter (EVOM2, World Precision Instruments) equipped with Endohm-12 chamber electrodes was used to measure transendothelial electrical resistance (TEER). Metabolic activity was determined using a WST1 assay (Cayman) according to the manufactures protocol, after exposure to increasing concentrations of cuprizone $(0-250 \mu \mathrm{M})$ for up to $72 \mathrm{~h}$, or $20 \mu \mathrm{M}$ peroxide as positive control.

\section{Expression analyses}

Expression analyses were done as described [11]. For tissue expression analyses, corpus callosum and cortex was dissected from Bregma +1.10 to -2.46 . RNA was extracted using QIAshredder and RNeasy protocols (Qiagen). Concentration and quality of RNA was evaluated using a NanoDrop spectrophotometer and RNA Nano (Agilent). cDNA was synthesized with Superscript III (Invitrogen) and quantitative PCRs were done in triplicates with the GoTaq pPCR Master Mix (Promega) on a 7500 Fast RealTime PCR System (Applied Biosystems). Expression values were normalized to the mean of two housekeeping genes, HPRT (Hypoxanthin-Phosphoribosyl-Transferase 1) and Rplp0 (60S acidic ribosomal protein P), and quantification was done by applying the $\Delta \Delta \mathrm{Ct}$ method, normalized to age matched untreated controls (set to 1). All primers (Additional file 1: Table S1) were intron-spanning.

\section{Antibodies}

CAII (Said Ghandour), Olig2 (Charles Stiles/ John Alberta), GFAP (Chemicon), MAC3 (Pharmigen), Iba1 (Wako), PECAM1 (dianova), AQP4 (Santa Cruz), occludin, ZO-1 and claudin-5 (Thermo Fisher), GAPDH
(Enzo). For generation of GLUT1 antisera, rabbits were immunized with the C-terminal intracellular peptide (CDKTPEELFHPLGADSQV). Anti-GLUT1 antibody was purified by affinity chromatography.

\section{Statistical analysis}

Statistical analysis was performed using Prism software (GraphPad Software), and results are presented as the mean \pm s.e.m.. Two-way ANOVA, one-way ANOVA and two-tailed unpaired Student's t tests were performed as appropriate. Only $P$ values $<0.05$ were considered statistically significant $\left({ }^{*} P<0.05,{ }^{* *} P<0.01,{ }^{* * *} P<0.001\right)$.

\section{Results}

Vascular permeability is increased at the peak of cuprizone induced demyelination

At the peak of demyelination after 5 weeks of cuprizone exposure, vascular permeability is increased as we showed previously [11]. To explore potential causes for this BBB instability, we performed a series of expression analyses on dissected corpus callosum samples from mice fed cuprizone for 5 weeks. As expected, histopathology (Additional file 2: Figure S1a) was reflected in reduced expression of oligodendroglial genes, e.g. Olig2 (oligodendrocyte lineage transcription factor 2) and increased expression of markers for gliosis (Gfap, glial fibrillary acidic protein; Aif1, allograft inflammatory factor 1, encoding Iba1) (Fig. 1a, Additional file 1: Table S2). When we quantified the levels of inflammatory mediators that have previously been shown to induce BBB dysfunction [42, 47, 58], Tnf (tumor necrosis factor), Il1b (interleukin 1 beta), and $\mathrm{Ccl} 2$ (C-C Motif Chemokine Ligand 2) were strongly upregulated (Fig. 1b). Other factors such as Il2 (interleukin 2) and Ifng (interferon gamma) remained unchanged compared to controls. Notably, BBB impairment in the cuprizone model was not associated with mRNA upregulation of HIF1 $\alpha$ (hypoxia induced factor 1 alpha), VEGF-A (vascular endothelial growth factor A) or matrix metalloproteinases, which are jointly associated with hypoxia-induced $\mathrm{BBB}$ dysfunction in EAE and other disease models $[5,6]$ (not shown). In contrast, mRNA abundance of the nitric oxide synthases NOS2 and NOS3 was significantly increased in cuprizone treated animals, implicating reactive nitrogen species likely mediators of BBB leakage. Consistent with these findings, expression of endothelial markers including genes encoding tight and adherens junction proteins PECAM1 (platelet/endothelial cell adhesion molecule 1/CD31, Pecam1 gene), claudin-5 (Cldn5), occludin (Ocln), ZO1 (Tjp1 gene), cadherin-1 (Cdh1), and cadherin-5 (Cdh5) were strongly downregulated (Fig. 1a).

When we examined the morphology of the BBB by transmission electron microscopy, cuprizone fed animals 


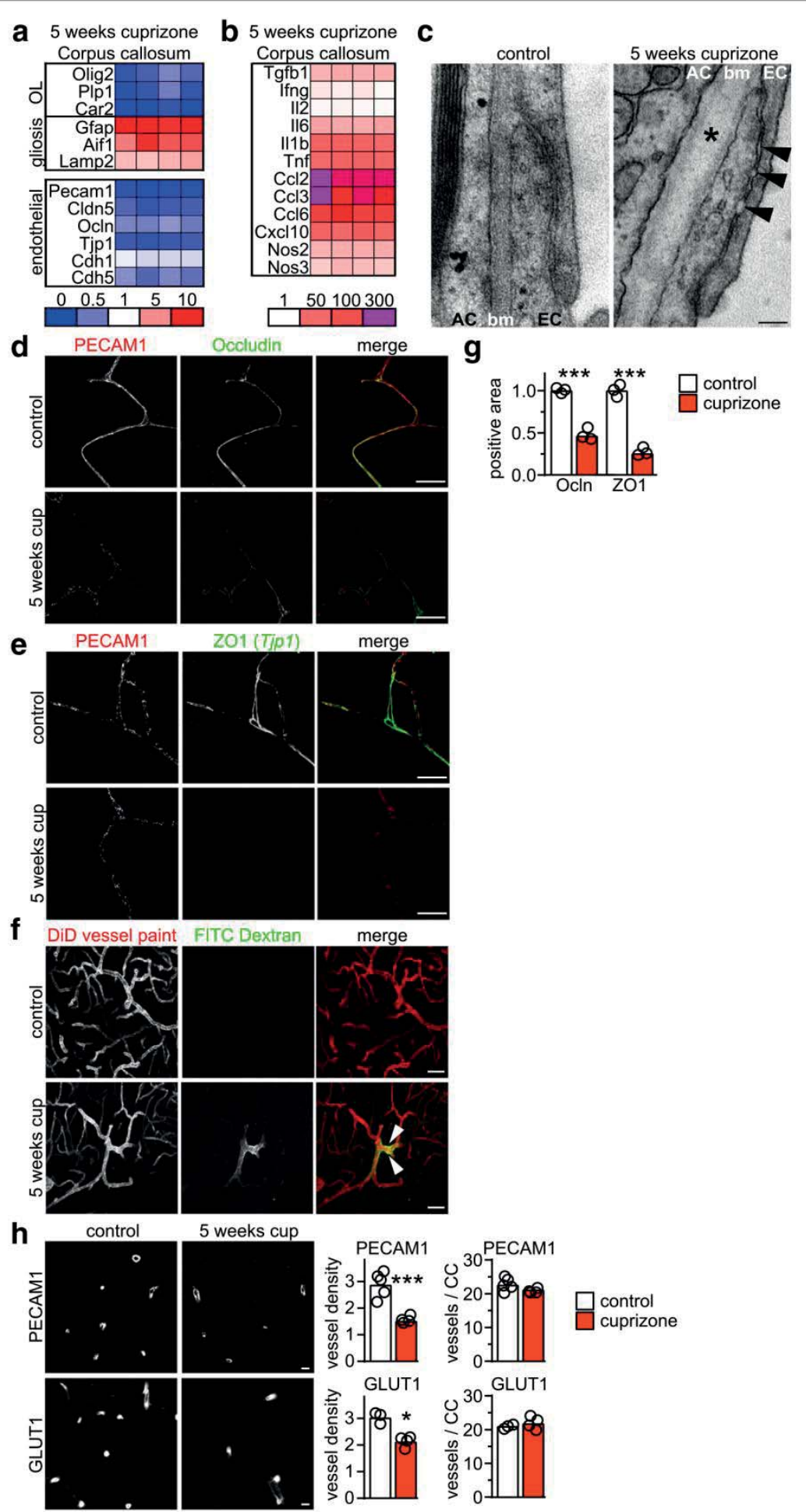

Fig. 1 (See legend on next page.) 


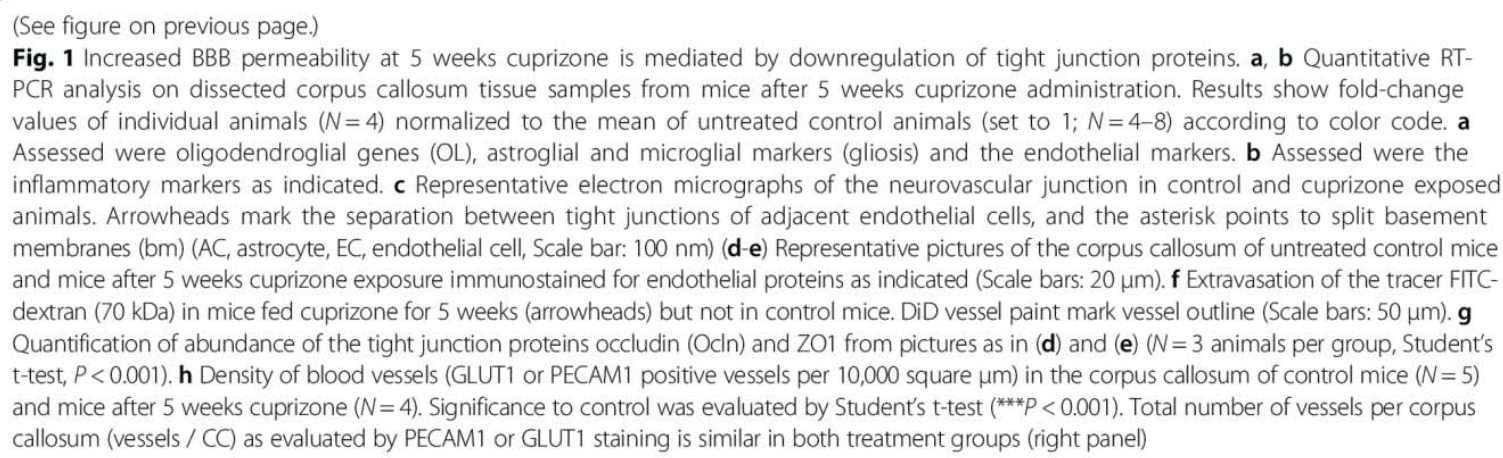

Fig. 1 Increased BBB permeability at 5 weeks cuprizone is mediated by downregulation of tight junction proteins. a, b Quantitative RTPCR analysis on dissected corpus callosum tissue samples from mice after 5 weeks cuprizone administration. Results show fold-change values of individual animals $(N=4)$ normalized to the mean of untreated control animals (set to $1 ; N=4-8$ ) according to color code. a Assessed were oligodendroglial genes (OL), astroglial and microglial markers (gliosis) and the endothelial markers. b Assessed were the inflammatory markers as indicated. c Representative electron micrographs of the neurovascular junction in control and cuprizone exposed animals. Arrowheads mark the separation between tight junctions of adjacent endothelial cells, and the asterisk points to split basement membranes $(\mathrm{bm})$ (AC, astrocyte, EC, endothelial cell, Scale bar: $100 \mathrm{~nm}$ ) (d-e) Representative pictures of the corpus callosum of untreated control mice and mice after 5 weeks cuprizone exposure immunostained for endothelial proteins as indicated (Scale bars: $20 \mu \mathrm{m}$ ). $\mathbf{f}$ Extravasation of the tracer FITCdextran $(70 \mathrm{kDa})$ in mice fed cuprizone for 5 weeks (arrowheads) but not in control mice. DiD vessel paint mark vessel outline (Scale bars: $50 \mu \mathrm{m})$. $\mathbf{g}$ Quantification of abundance of the tight junction proteins occludin (Ocln) and ZO1 from pictures as in (d) and (e) ( $\mathrm{N}=3$ animals per group, Student's t-test, $P<0.001)$. $\mathbf{h}$ Density of blood vessels (GLUT1 or PECAM1 positive vessels per 10,000 square $\mu \mathrm{m})$ in the corpus callosum of control mice $(\mathrm{N}=5$ ) and mice after 5 weeks cuprizone $(N=4)$. Significance to control was evaluated by Student's t-test $(* * * P<0.001)$. Total number of vessels per corpus callosum (vessels / CC) as evaluated by PECAM1 or GLUT1 staining is similar in both treatment groups (right panel)

often displayed a discontinuous electron-dense junctional area with focally increased junctional width (Fig. 1c, Additional file 2: Figure S2), implying that the physical barrier of the $\mathrm{BBB}$ mediated by endothelial tight and adherens junctions could be altered. This observation was confirmed by immunofluorescence analysis of selected tight junction proteins that showed strongly reduced staining intensity in cuprizone fed animals (Fig. 1d-g). Increased BBB permeability was demonstrated by extravasation of tracers such as FITC-dextran (Fig. 1f, see also below). By electron microscopy we also observed locally split endothelial and astroglial basement membranes, hypertrophic astrocytes, and sporadic endothelial cells with atypical ultrastructure in cuprizone fed animals (Fig. 1c, Additional file 2: Figure S2). Moreover, while the number of blood vessels in the entire cross-sectional area of the corpus callosum remained unchanged, vessel density was strongly reduced (Fig. 1h), likely caused by the tissue swelling due to the dramatic gliosis at this time point (Additional file 2: Figure S1). Together, these data show that the increased $\mathrm{BBB}$ permeability at the peak of cuprizone induced demyelination coincides with substantial upregulation of BBB-disrupting pro-inflammatory mediators. This is associated with strong downregulation of endothelial tight junction proteins and morphological changes at the endothelial barrier.

\section{Cuprizone directly affects BBB permeability in vitro}

We next explored whether cuprizone directly damages cellular constituents of the NVU, namely mouse brain endothelial cells or astrocytes, as observed for mature oligodendrocytes [10]. Using primary cultures of either cell type in a WST1 assay that measures the activity of cellular dehydrogenases, cuprizone did not affect metabolic activity within $24 \mathrm{~h}$, even when administered in concentrations up to $500 \mu \mathrm{M}$ (Additional file 2: Figure S3a, b). However, after $72 \mathrm{~h}$ incubation with $250 \mu \mathrm{M}$ cuprizone, the WST1 signal in endothelial cells decreased by about $14 \%$ compared to vehicle treated cultures (Additional file 2: Figure S3c, d), which were not caused by cell death but rather reflected metabolic adaptations to the toxic cuprizone insult.

To investigate the effect of cuprizone on the barrier function of endothelial cells in more detail, we analyzed transendothelial electrical resistance (TEER) in an in vitro BBB system of endothelial monocultures. Surprisingly, cuprizone significantly decreased TEER after only $48 \mathrm{~h}$ that dropped further to about $80 \pm 3 \%$ (mean \pm SD) of control values after $72 \mathrm{~h}$ (Fig. 2a). This effect was also observed in the advanced BBB setup, in which endothelial cells were co-cultured with primary astrocytes (Fig. 2b). Moreover, by immunostaining and expression analysis of primary endothelial cells, we observed reduced protein and mRNA abundance of the tight junction protein occludin, and reduced Abcb1a mRNA of the ABCB1 (P-glycoprotein) efflux transporter, likely explaining the decreased tightness of the barrier in vitro (Fig. 2c, d). Abundance of mRNAs for the astroglial BBB maintenance factor sonic hedgehog and for other tight junction proteins, which were strongly downregulated in the corpus callosum of cuprizone treated animals (Fig. 1a, d, e), remained unchanged. Cuprizone treatment downregulated occludin in the absence of inflammatory mediators, whose expression in primary endothelial, astrocyte, and microglial cultures were not induced by the cuprizone challenge (Additional file 2: Figure S3e-g). Together, these in vitro data suggest that cuprizone could directly affect BBB constituents in vivo, even in the absence of additional disease processes.

\section{BBB dysfunction depends on local pathology}

To test this, we treated mice for 5 weeks with cuprizone as before and then analyzed the integrity of endothelial tight junctions on cortical sections. The cortex develops the demyelinating pathology later than the corpus callosum (Additional file 2: Figure S1), as previously reported $[13,23]$, allowing us to test if tight junction integrity is affected before pathology exacerbates. In the cortex of cuprizone treated animals and controls, staining intensity and continuity of the tight junction proteins occludin and 

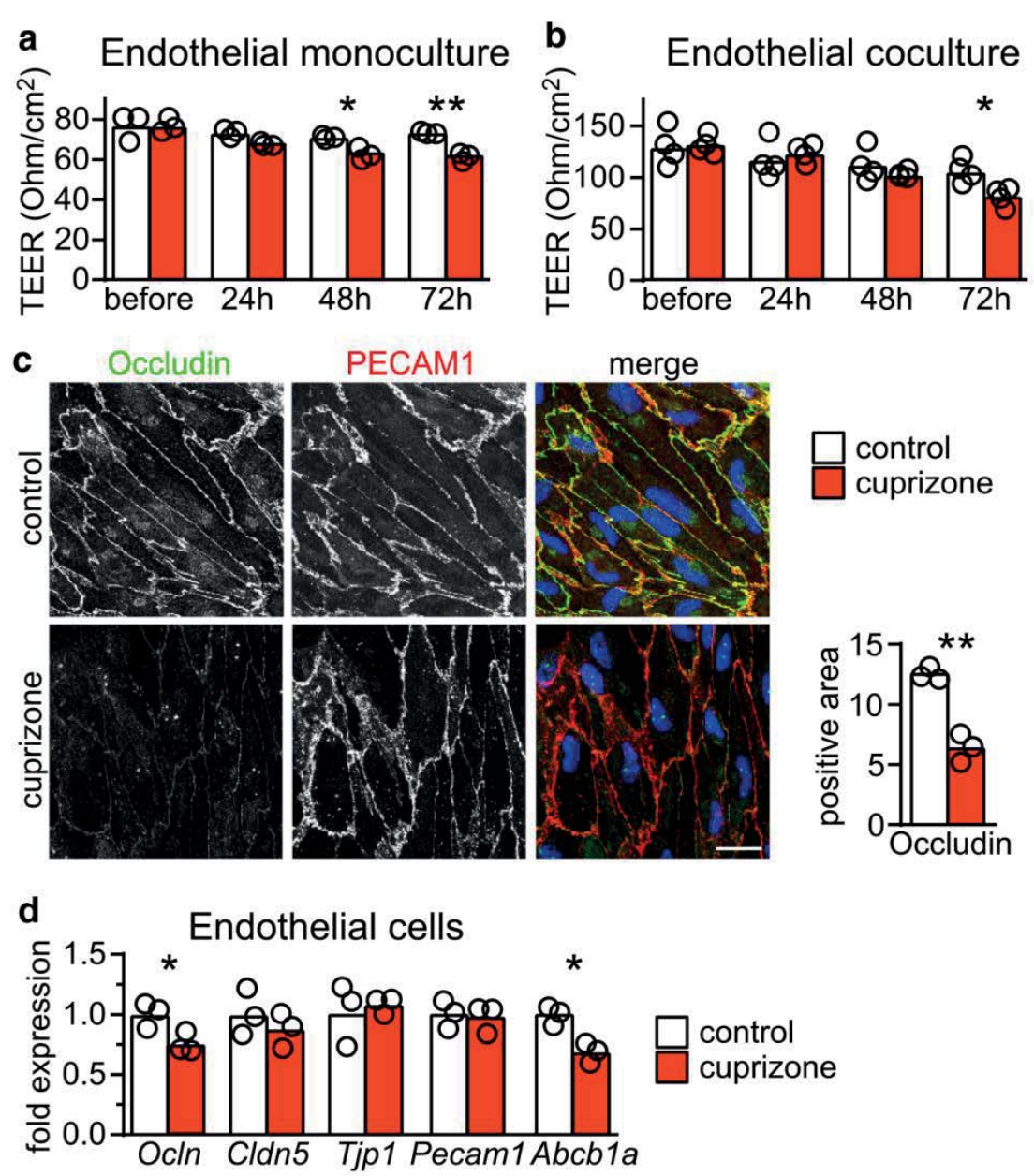

Fig. 2 Cuprizone increases BBB permeability in vitro. Transendothelial electrical resistance (TEER) of (a) monocultures of primary endothelial cells (EC) and (b) co-cultures of EC with astrocytes after $250 \mu \mathrm{M}$ cuprizone for $24 \mathrm{~h}, 48 \mathrm{~h}$ and $72 \mathrm{~h}$. Evaluation of significance was done by 2 way ANOVA with Sidak's post test $\left({ }^{*}, P<0.05 ;{ }^{* *}, P<0.01\right)$. Shown is one representative experiment but similar results were obtained in three additional experiments (each $N=3-4$ mice per condition). c Immunostaining of EC for occludin and PECAM1 that had been treated $250 \mu \mathrm{M}$ cuprizone for $48 \mathrm{~h}$ (Scale bar: $20 \mu \mathrm{m})$ with quantification of $\%$ anti-occludin positive area on the right $\left(N=3\right.$ independent experiments, Student's t-test, $\left.P>0.01{ }^{* *}\right)$. d Quantitative RT-PCR analysis on EC challenged with $250 \mu \mathrm{M}$ cuprizone for $48 \mathrm{~h}$. Results show mean fold changes of individual cultures $(N=3)$ normalized to vehicle treated controls (set to 1). Significance to control was evaluated by unpaired Student's t-test $\left(P<0.05^{*}\right)$

ZO1 was similar (Fig. 3a, b), in contrast to the strongly reduced staining intensity in corpus callosum of the same animals (Fig. 1d-g). Similarly, abundance of the tight junction protein claudin-5 (Fig. 3c) was not significantly reduced in cortex $(90 \pm 2 \%$ of controls in cortex lysates) of cuprizone fed mice in comparison to corpus callosum lysates $(44 \pm 4 \%$ of controls, $n=3,2$-way ANOVA $P<$ 0.0001 ). Of note, abundance of claudin- 5 in control animals was $69 \pm 3 \%$ in cortex compared to corpus callosum $(n=3, n=3,2$-way ANOVA $P<0.0001)$, in line with a study using porcine brain [41]. In accordance with the mild gliosis (Additional file 2: Figure S1), vessel density remained unchanged in cortex of cuprizone treated mice compared to controls (Fig. 3d). These findings suggest that in vivo, cuprizone alone is not sufficient to completely abolish BBB integrity (see also below).

To substantiate the heterogeneity of $\mathrm{BBB}$ pathology with another marker for NVU integrity, we compared Aquaporin 4. (AQP4) distribution in corpus callosum and cortex by immunostaining. AQP4 localization was restricted to astrocyte endfeet in control animals and the cortex of mice up to 5 weeks cuprizone (Fig. 3e, f), suggesting this brain region intact with respect to BBB integrity. In contrast, in the corpus callosum of these mice, AQP4 displayed a diffuse staining pattern, and Aqp4 mRNA expression was significantly upregulated 
Publications/Manuscripts
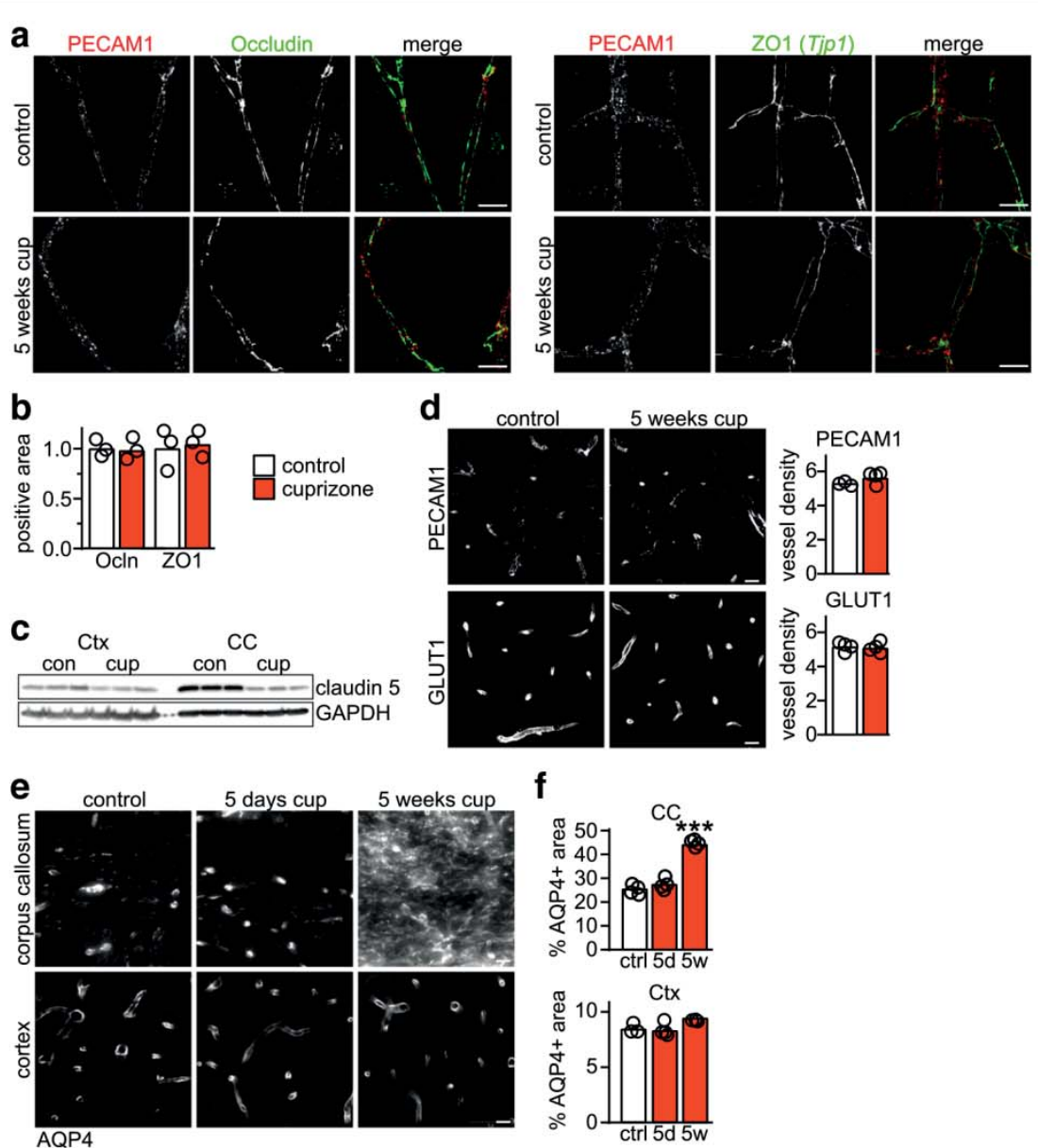

Fig. 3 BBB dysfunction depends on local pathology. a Representative pictures of the cortex of untreated control mice and mice after 5 weeks cuprizone exposure immunostained for endothelial tight junction proteins as indicated co-labeled with the endothelial marker PECAM1 (Scale bars: $20 \mu \mathrm{m}$ ). b Quantification of abundance of the tight junction proteins occludin (Ocln) and ZO1 from pictures as in (a). c Immunoblot detecting claudin-5 in cortex (Ctx) and corpus callosum (CC) lysates from mice treated with cuprizone (cup) for 5 weeks and untreated controls. GAPDH shows equal loading of protein. $\mathbf{d}$ Immunostaining and density of blood vessels (PECAM1 or GLUT1 positive vessels per square $\mathrm{mm}$ ) in the cortex of control mice $(N=5)$ and mice after 5 weeks cuprizone $(N=4)$. e Representative pictures of corpus callosum and cortex of untreated control mice and mice after 5 weeks cuprizone immunostained for AQP4 (Scale bar: $20 \mu \mathrm{m}$ ). f Quantification of immunolabeling as depicted in (e) of $N=3-4$ animals per condition. Significance was evaluated by 1 way ANOVA with Dunnett's post test (***P<0.001)

( $2.71 \pm 0.01$ fold, $n=4$ animals per group, $P<0.0001$, Student's t-test), a condition also found in MS lesions [50] and typical for cerebral edema in EAE. Together these findings suggest that the morphological disturbances at the $\mathrm{BBB}$ are associated with local active disease in cuprizone fed animals. This prompted us to investigate at which disease state BBB dysfunction develops.

\section{BBB impairment and edema are very early disease} processes

To determine the temporal progression of BBB impairment, we simultaneously assessed the degree of BBB permeability and vasogenic edema after 3 days to 5 weeks of cuprizone administration (Fig. 4a). Brain water as a measure of edema was already significantly evident after 3 days cuprizone and progressively increased (Fig. 4b). BBB dysfunction, as assessed by the biochemical extraction of extravasated Evans blue or sodium fluorescein (Fig. 4a), developed with a similar time course as edema, demonstrating a significant $1.25 \pm 0.02$ fold extravasation (mean \pm s.e.m., $n=4$ ) after 3 days of cuprizone (Fig. 4c, d). Because global edema values could be misleading, we determined water content individually, in corpus callosum and cortex of the same animals after 5 days of cuprizone exposure. While the water content in the cortex of cuprizone treated animals remained in the same 
Publications/Manuscripts
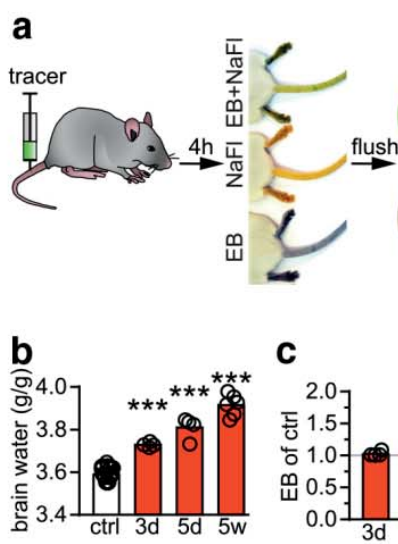

C
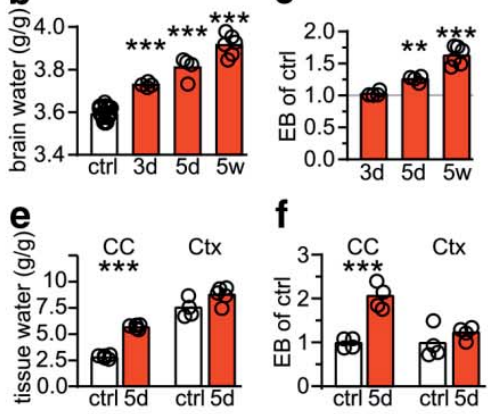

f

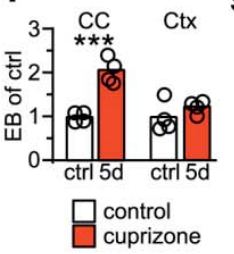

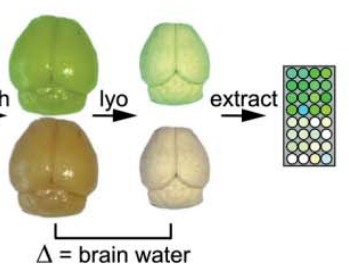

d
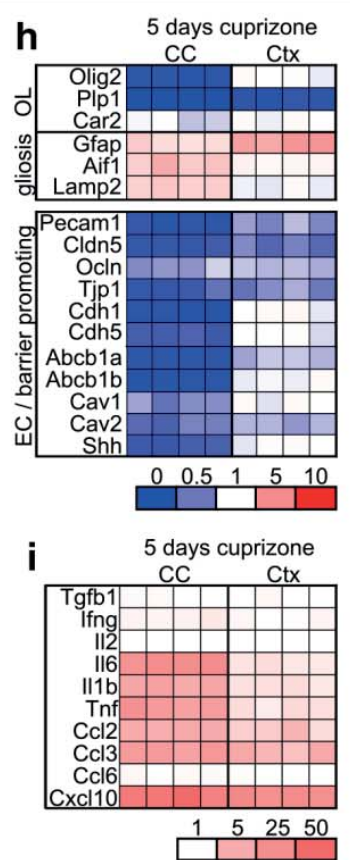

Fig. 4 Cuprizone induces early BBB dysfunction and edema. a Experimental procedure to measure BBB permeability by quantifying extravasation of the tracers Evans blue (EB) and sodium fluorescein ( $\mathrm{NaFl}$ ), and edema (brain water). Tracers are i.v. injected, and mice are flushed with PBS after $4 \mathrm{~h}$. The dissected brain is lyophilized (lyo). Brain water content (edema) is calculated from the weight difference of wet to lyophilized tissue. Tracers are extracted, and fluorescence intensity quantified. b Brain water ( $\mathrm{g} / \mathrm{g}$ dry brain) in mice fed cuprizone for 3 days to 5 weeks as indicated, and untreated controls $(N=4-5)$. Significance to control was evaluated by 1 way ANOVA with Tukey's post test (***P $<0.001)$. c, d Extravasated EB ( $N=4-6$ ) and $\mathrm{NaFI}(\mathrm{N}=4)$ after cuprizone administration for 3 days to 5 weeks. Significance to control was evaluated by 1way ANOVA with Tukey's post test $\left({ }^{* *} P<0.01,{ }^{* * *} P<0.001\right)$. e Water content ( $\mathrm{g} / \mathrm{g}$ dry tissue) in dissected corpus callosum (CC) or cortex (Ctx) of mice fed cuprizone for 5 days or controls $(N=4-5)$. Significance was determined by unpaired Student's t-test for each brain region $\left.{ }^{* * *} P<0.001\right)$. Extravasated $(\mathbf{f}) \mathrm{EB}(N=4)$ and $(\mathbf{g}) \mathrm{NaFI}(N=5)$ in dissected CC or Ctx of mice fed cuprizone for 5 days normalized to untreated controls. Significance to controls was evaluated by Student's t-test ( ${ }^{*} P$ $<0.05$, $\left.{ }^{* * *} P<0.001\right)$. Quantitative RT-PCR analysis on dissected CC or Ctx from mice that received cuprizone for 5 days $(N=4)$ or control mice, evaluating cellular markers (h) and inflammatory mediators (i). Values of individual mice are shown as fold differences to the mean of $\mathrm{N}=4-5$ controls (set to 1) according to color code

range as in controls, the corpus callosum of cuprizone treated animals displayed a $16.6 \pm 0.5 \%$ water increase (Fig. 4e, mean \pm s.e.m., $N=4-5$ ), a value typical for focal edema [28]. Corresponding with these local differences in edema, focal extravasation of fluorescent tracers increased more than twofold in the corpus callosum but only marginally in the cortex of cuprizone treated animals (Fig. 4f, g).

To assess how this regional heterogeneity of edema and BBB permeability correlates with local pathology and gliosis in this early disease phase, we performed a series of quantitative expression analyses on dissected corpus callosum and cortex together with histopathological evaluation of tissue sections, after 5 days of cuprizone. Expression of marker proteins for astrocytes and microglia reflected the mild histopathological changes (Fig. 4h, Additional file 1: Table S3, Additional file 2: Figure S1). Despite reduced Plp1 and Olig2 expression, mice lacked signs of demyelination and loss of oligodendrocytes, as reported previously
$[13,24]$. The abundance of mRNAs of endothelial tight junction proteins were only moderately downregulated in cortex compared to corpus callosum, mirroring the immunostaining data at 5 weeks of cuprizone exposure (Fig. 4h, compare Figs. 1d-e, 3a). Expression of the ABCB1 transporter and associated caveolins as well as of the BBB maintenance factor sonic hedgehog remained almost unaffected in the cortex compared to untreated wild type mice. It is possible that a direct effect of cuprizone contributed to the residual downregulation of endothelial markers and to the slight BBB hyperpermeability (Fig. 4g), similar to our observations in vitro (compare Fig. 2). In contrast, all markers for NVU integrity were strongly downregulated in corpus callosum at this early time point of cuprizone induced pathology.

Levels of the inflammatory mediators $I l 6, I l 1 b, T n f$, and $\mathrm{Ccl} 2$ were strongly elevated in the corpus callosum but only moderately increased in the cortex after 5 days of cuprizone (Fig. 4i). These data demonstrate that the 
BBB integrity is already affected within the first days of cuprizone exposure, coinciding with elevated levels of inflammatory mediators but preceding overt demyelination and oligodendrocyte loss.

\section{Reduced inflammation ameliorates BBB pathology}

These findings prompted us to test directly whether demyelination and oligodendrocyte loss or local gliosis and the secretion of inflammatory mediators correlate with $\mathrm{BBB}$ dysfunction. Therefore, we used type $3 \mathrm{CXC}$ chemokine receptor (CXCR3) deficient mice [26] that develop demyelination in response to cuprizone as wild type mice but show strongly reduced reactive gliosis and expression of pro-inflammatory cytokines and chemokines such as TNF, IL6 and CCL2 [32].

After 5 days of cuprizone, we found attenuated expression of markers for astrogliosis and microgliosis as well as inflammatory mediators in CXCR3 deficient corpus callosum compared to identically treated wild type animals (Fig. 5a, b). Expression of the oligodendroglial transcription factor Olig2 and the myelin protein PLP1 was ameliorated in CXCR3 deficient mice (Olig2, $3.86 \pm 0.23$ fold; Plp1, $2.28 \pm 0.05$ fold in CXCR3 knockout mice compared to cuprizone fed controls), suggesting that the oligodendroglial damage was slightly less severe at this time point. Interestingly, the strong downregulation of genes indicative of $\mathrm{BBB}$ dysfunction such as tight junction proteins and $\mathrm{BBB}$ maintenance factors was also ameliorated in CXCR3 deficient animals (Fig. 5c). Reduced brain edema (Fig. 5d) and attenuated extravasation of fluorescent tracers (Fig. 5e, f) in CXCR3 deficient animals further support the hypothesis that proinflammatory mediators contribute to BBB disruption in response to cuprizone exposure.

Although CXCR3 is mainly expressed by microglial cells in untreated mice [26], and also when mice are challenged with cuprizone (Additional file 2: Figure S4), the cell type responsible for establishing the cytokine milieu during initial cuprizone pathology that contributes to BBB dysfunction is unknown. Therefore, we acutely isolated microglia, astrocytes, oligodendrocytes, and endothelial cells from wild type and CXCR3 deficient mice after 5 days of cuprizone treatment and from untreated wild type control animals, and analyzed mRNA abundance of Tnf, $I l 1 b, I l 6$, and $\mathrm{Ccl} 2$. We chose these inflammatory mediators because their expression pattern correlates with the extent of BBB disturbances: after 5 days of cuprizone, their expression levels were most strongly increased in corpus callosum of wild type mice, moderately increased in the corpus callosum of CXCR3 mutant animals, and only weakly upregulated in the cortex of wild type animals compared to untreated wild type controls (compare Figs. 4i and $5 \mathrm{~b}$ ). Oligodendroglia did not significantly contribute to the cytokine and chemokine profile after 5 days of cuprizone and surprisingly, neither did microglia (Fig. 5g, Additional file 1: Table S4). Endothelial cells showed moderate upregulation of cytokine and chemokine expression. In contrast, we identified astrocytes as the major source of all tested pro-inflammatory mediators at this early disease phase (Fig. 5g). Further, the increased expression of inflammatory molecules was completely abolished in astroglia of CXCR3 deficient mice, suggesting that microglial CXCR3 signaling induces astroglial upregulation of cytokines and chemokines in response to cuprizone.

\section{Discussion}

BBB impairment is considered as an important feature in MS pathologies, but the causal relation to disease processes during initial lesion formation and its impact on disease activity is unknown. In the current study we analyzed the temporal and spatial relationship of BBB dysfunction to gliosis, expression of inflammatory mediators, and demyelinating pathology in the cuprizone mouse model of demyelination. By using wild type and CXCR3 deficient mice, we demonstrate that BBB impairment is most pronounced in the corpus callosum of wild type animals, to a lesser extent in the corpus callosum of CXCR3 deficient mice, and only minimally in the cortex of wild type mice in the initial disease phase before the onset of demyelination. Our data indicate that IL6, IL1 $\beta$, TNF, and CCL2 are the most likely candidates to contribute to BBB dysfunction in the cuprizone model.

It is well known that TNF, IL6, IL1 $\beta$ and CCL2 can each induce downregulation of endothelial tight junction proteins in vitro and induce BBB hyperpermeability in vivo $[14,20,43,47,53,58]$. Enhanced paracellular leakage is mediated by downregulation of mRNA of tight junction proteins as shown in our study and in a model of bacterial infection [40]. Pro-inflammatory cytokines induce signaling cascades that can lead to downregulation of stabilizing factors such as sonic hedgehog $[3,58]$, to activation of effector proteins such as matrix metalloproteinases [33], and to the formation of reactive oxygen species [47]. In our experimental paradigm, increased expression of nitric oxide synthases and downregulated sonic hedgehog signaling likely contributed to tight junction disruption. In addition, all three cytokines have the ability to reduce $\mathrm{ABCB} 1$ mediated efflux, facilitating transendothelial passage [25]. We found downregulation of $\mathrm{ABCB} 1$ not only in cuprizone fed mice but also in endothelial cultures in the absence of inflammatory mediators, suggesting that cuprizone directly affected transendothelial passage.

Although it is generally assumed that microglia secrete the majority of effector molecules, our data show that in the initial disease phase of the cuprizone model microglia do not themselves contribute to the upregulation of IL6, IL1 $\beta$, TNF or CCL2; rather astrocytes (with a 

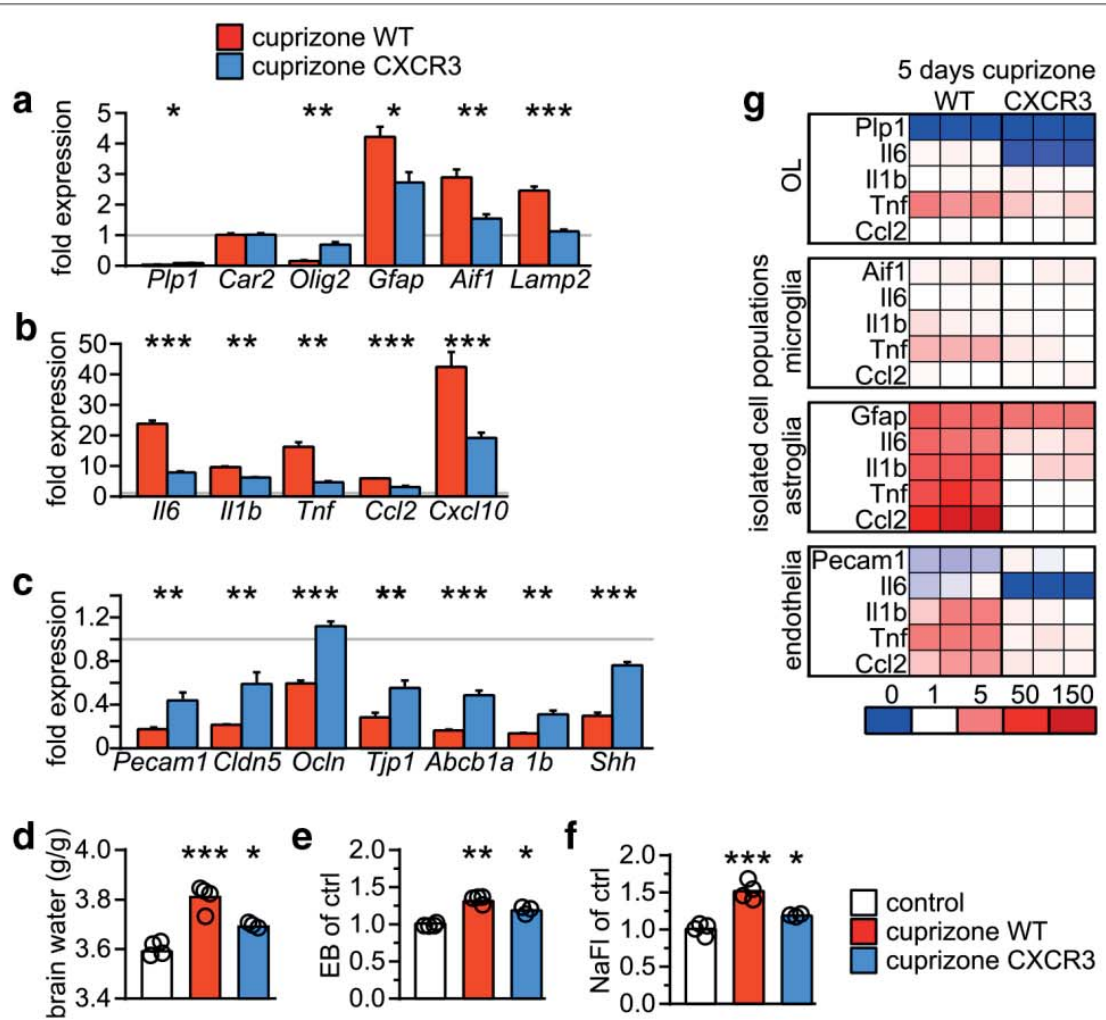

Fig. 5 CXCR3 KO ameliorates BBB pathology. a-c Quantitative RT-PCR analysis on dissected corpus callosum tissue samples from wild type (red) or CXCR3 deficient (blue) mice after 5 days cuprizone administration or untreated control mice. Results are expressed as mean fold change of $\mathrm{N}=3-4$ mice \pm s.e.m., normalized to untreated wild type control animals (set to 1; gray line, $N=5$ ). Assessed were (a) cellular markers, (b) cytokines and chemokines, and (c) endothelial and BBB promoting markers as indicated. Significance between WT mice and CXCR3 mice was evaluated by Student's t-test for each individual gene $\left({ }^{*} P<0.05,{ }^{* *} P<0.01,{ }^{* *} P<0.001\right)$. $\mathbf{d}$-f Brain water ( $\mathrm{d}, \mathrm{g} / \mathrm{g}$ dry brain), of extravasated EB (e), and $\mathrm{NaFl}(\mathbf{f})$ in untreated wild type controls (white, $N=3-4$ ), and wild type (red, $N=3-4$ ) or CXCR3 deficient (blue, $N=3$ ) mice exposed to cuprizone for 5 days. Significance to control was evaluated by 1 way ANOVA with Tukey's post test $\left({ }^{*} P<0.05,{ }^{* *} P<0.01\right.$, $\left.{ }^{* * *} P<0.001\right)$. g Quantitative RT-PCR on isolated cells as indicated from brain of from WT or CXCR3 deficient mice after 5 days cuprizone administration or untreated control mice. Results show fold-change values according to color code of individual animals $(N=3)$ normalized to the mean of untreated control animals (set to $1 ; N=5)$

moderate participation by endothelial cells) are the main source of these pro-inflammatory cytokines and chemokines. At later disease stages, however, microglia substantially add to the production of inflammatory factors [57]. Astrocytes participate in recruiting microglia as shown in mouse mutants with acutely depleted astrocytes [52]. They promote recovery and repair in mouse models of remyelination but can also facilitate demyelination in acute active lesions of MS patients [11, 31, 35]. Whether astrocytes are the main source of disease promoting factors in presymptomatic MS patients before the onset of demyelination is unknown. We demonstrate that astrocytes, which are intimately involved in regulating BBB function via their endfeet, create a local inflammatory milieu that likely participates in destabilizing BBB integrity. Although cuprizone by itself does not induce astroglial, endothelial, or microglial expression of any of the tested pro-inflammatory mediators in vitro, it mildly affects metabolic activity in endothelial cells (this study) in addition to mature oligodendrocytes [10]. Death of oligodendrocytes in vivo is enforced by local glial activation [27]. We hypothesize that also vascular cells contribute to this complex crosstalk that lead to BBB impairment and demyelination in the cuprizone model.

Paracellular influx of fluid because of BBB disruption is the leading cause of vasogenic edema [54], also found in MS and inflammatory models of MS [9, 48, 60]. Edema correlates with increased AQP4 abundance and its mislocalization from (frequently hypertrophic) astroglial endfeet that is associated with altered basement membrane morphology in EAE [2, 60]. We also observed loosening of the astrocytic and endothelial basement membranes in the cuprizone model, emphasizing similar pathogenic processes in these disparate models of MS. Although it is well-known that cuprizone intoxication causes spongiform degeneration of the CNS [15], edema has been largely 
ignored, potentially because of the absence of massive BBB disruption in this model $[8,12,30]$. For the first time, to our knowledge, we quantified edema in the cuprizone model and found that increased brain water content was most pronounced during overt demyelination. Importantly, edema was already obvious before the onset of demyelination and oligodendrocyte loss.

In agreement with others [13, 23], we observed marked regional differences in disease manifestation in the cuprizone model. Interestingly, similar to our observation in the cortex of cuprizone treated animals, cortical pathology in MS also differs substantially from white matter lesions; the former comprising only mild gliosis and modest alteration of tight junctions [21, 56]. In addition to the differences in tissue architecture and expression profiles of neural cells [22,38], the regional heterogeneity of pathology could also be influenced by differences in vasculature. As we show here in accordance with previous studies, vessel density in gray matter is about double of that in white matter, and steady state levels of tight junction proteins are lower [41]. Together, these differences likely modify BBB properties, which might play a role in rendering cortical MS lesions less susceptible to disease exacerbation.

Does an impaired BBB directly affect the course of demyelinating disease? Chronic upregulation of inflammatory mediators directly impairs BBB integrity and can induce demyelinating pathology [46]; conversely, their genetic or pharmacological reduction can improve BBB function but their role in modulating severity of demyelinating disease in the context of EAE or cuprizone is less clear [7, 16, 36, 39]. The induction of an inflammatory milieu and BBB impairment have been uncoupled by inhibition of nitric oxide synthesis in the cuprizone model [29] or by targeted overexpression of claudin-1 in EAE [44] that both support BBB tightness but presumably do not (directly) affect expression of pro-inflammatory molecules. In these experimental paradigms, clinical symptoms of treated/transgenic mice were ameliorated, suggesting that endothelial integrity might contribute to disease expression. In a study with 39 patients with neuromyelitis optica, BBB leakage in normal appearing white matter correlated with progression to MS pathology [17]. We show here that BBB dysfunction and edema occurred before demyelination, suggesting that demyelination itself does not cause BBB damage. We speculate that $\mathrm{BBB}$ dysfunction might serve as a predictive marker for local disease activity.

\section{Conclusions}

In summary, our data show that in vitro cuprizone directly increases BBB permeability mediated by downregulation of tight junction proteins. In vivo, astroglial derived pro-inflammatory cytokines create a local inflammatory milieu that likely further destabilizes local
BBB integrity and induces edema. We envision that glial activation and production of pro-inflammatory mediators in any neurological disorder destabilizes BBB integrity. In demyelinating disease, these presymptomatic disease processes might have prospective value for future disease activity and disease progression.

\section{Additional files}

Additional file 1: Table S1. Primer sequences used for gene expression analyses. Table S2. Quantification of gene expression in corpus callosum. Table S3. Quantification of gene expression in cortex. Table S4. Quantification of gene expression in acutely isolated cells. (PDF $70 \mathrm{~kb}$ ) Additional file 2: Figure S1. Time course of cuprizone induced pathology in corpus callosum and cortex. (a) Representative pictures of the corpus callosum (CC) and the cortex $(\mathrm{Ct} \mathrm{x})$ of untreated control mice and mice after cuprizone exposure for 5 days and 5 weeks assessing myelination (Gallyas silver impregnation), mature oligodendrocytes (CAII), activated microglia (MAC3), and astrocytes (GFAP) (Scale bars: $20 \mu \mathrm{m}$ ) with quantification in (b). Each bar represents the mean value of $N=3-4$ animals per condition with individual data points. Significance to control was evaluated by 1 way ANOVA with Tukey's post test $\left({ }^{*} P<0.05,{ }^{* *} P<\right.$ $0.01,{ }^{* * *} P<0.001$ ). Figure S2. Endothelial junctions in mice after 5 weeks cuprizone. Electron microscopic images of capillaries showing disconnected endothelial and astroglial basement membranes (a arrowheads), an affected endothelial cell (b) with electron light cytoplasm and focal disruption of endothelial tight junctions ( $a, b$, arrow). Astroglial endfeet often appeared swollen (c, green). Scale bars: $2 \mu \mathrm{m}$ (AC,

astrocyte; EC, endothelial cell; EF, astroglial endfoot; $\mathrm{M}$, microglia/ macrophage; $P$, pericyte). Figure S3. Cuprizone affects endothelial cells but not astrocytes in vitro. Cell vitality measurements (WST1 assays) of primary $(a, c)$ endothelial cells or $(b, d)$ astrocytes after exposure to vehicle $(0$, white) or to increasing concentrations of cuprizone (50$500 \mu \mathrm{M}$, red) for $24 \mathrm{~h}(\mathrm{a}, \mathrm{b})$ or with $250 \mu \mathrm{M}$ cuprizone for $72 \mathrm{~h}(\mathrm{c}, \mathrm{d})(\mathrm{N}=$ 5 per condition). Incubation with $20 \mu \mathrm{M}$ peroxide for $4 \mathrm{~h}$ induced cell death and was used as positive control $(N=3,+)$. Significance to vehicle was evaluated by $(a, b) 1$ way ANOVA with Dunnett's post test or $(c, d)$ Student's t-test ( ${ }^{* *} P<0.01$, $\left.{ }^{* * *} P<0.001\right) .(\mathrm{e}, \mathrm{f}, \mathrm{g})$ Quantitative RT-PCR analysis on cultured primary endothelial cells (e), astrocytes (f), and microglia (g) challenged with $250 \mu \mathrm{M}$ cuprizone for $48 \mathrm{~h}$. Results show mean fold change with individual data points of $N=3$ cultures normalized to vehicle control (set to 1). Figure S4. CXCR3 is expressed by microglia in cuprizone fed mice. Direct GFP fluorescence of CXCR3 ${ }^{\text {GFP/GFP }}$ mice together with immunolabeling of cell type specific markers for microglia (lba1), astrocytes (GFAP), or oligodendroglia (Olig2) in the corpus callosum and cortex in CXCR3 deficient mice that had been exposed to cuprizone for 5 days (Scale bars: $20 \mu \mathrm{m}$ ). (PDF $19968 \mathrm{~kb}$ )

\section{Acknowledgements}

We are grateful to Klaus-Armin Nave for constant support. We thank Silvia Thüne, Annette Fahrenholz, Martin Meschkat, and Beatriz Salas Vegue for technical support. We thank Iva Tzetanova for providing primary microglial cells. We cordially thank Julia Edgar for editing and critical discussions. This study was funded by the Deutsche Forschungsgemeinschaft (SA 2014/2-1 to GS). WM was funded by an ERC Advanced grant awarded to Klaus-Armin Nave. All authors read and approved the final manuscript.

\section{Availability of data and materials}

All data generated or analyzed during this study are included in this published article (and its Additional files 1 and 2).

\section{Authors' contributions}

GS, and $S A B$ conceptualized the study and designed the experiments, $S A B$, TD, JW, SKS, NG, LS, TR, BHC, WM performed experiments. KK generated the GLUT1 antibody. SAB and GS wrote the manuscript. All authors read and approved the final manuscript. 
Competing interests

The authors declare that they have no competing interests.

\section{Publisher's Note}

Springer Nature remains neutral with regard to jurisdictional claims in published maps and institutional affiliations.

\section{Author details}

${ }^{1}$ Department of Neurogenetics, Max-Planck-Institute of Experimental Medicine, Hermann-Rein-Str. 3, 37075 Goettingen, Germany. ${ }^{2}$ Center Nanoscale Microscopy and Molecular Physiology of the Brain (CNMPB), Wilhelmsplatz 1, 37073 Göttingen, Germany.

Received: 12 October 2017 Accepted: 17 November 2017

Published online: 01 December 2017

\section{References}

1. Abbott NJ, Patabendige AA, Dolman DE, Yusof SR, Begley DJ (2010) Structure and function of the blood-brain barrier. Neurobiol Dis 37:13-25

2. Agrawal $S$, Anderson $P$, Durbeej $M$, van Rooijen $N$, Ivars F, Opdenakker $G$, Sorokin LM (2006) Dystroglycan is selectively cleaved at the parenchymal basement membrane at sites of leukocyte extravasation in experimental autoimmune encephalomyelitis. J Exp Med 203:1007-1019

3. Alvarez Jl, Dodelet-Devillers A, Kebir H, Ifergan I, Fabre PJ, Terouz S, Sabbagh M, Wosik K, Bourbonniere L, Bernard M, Van HJ, De Vries HE, Charron F, Prat A (2011) The hedgehog pathway promotes blood-brain barrier integrity and CNS immune quiescence. Science 334:1727-1731

4. Alvarez J, Saint-Laurent O, Godschalk A, Terouz S, Briels C, Larouche S, Bourbonniere L, Larochelle C, Prat A (2015) Focal disturbances in the bloodbrain barrier are associated with formation of neuroinflammatory lesions. Neurobiol Dis 74:14-24

5. Argaw AT, Asp L, Zhang J, Navrazhina K, Pham T, Mariani JN, Mahase S, Dutta DJ, Seto J, Kramer EG, Ferrara N, Sofroniew MV, John GR (2012) Astrocyte-derived VEGF-A drives blood-brain barrier disruption in CNS inflammatory disease. J Clin Invest 122:2454-2468

6. Argaw AT, Zhang Y, Snyder BJ, Zhao ML, Kopp N, Lee SC, Raine CS, Brosnan CF, John GR (2006) IL-1beta regulates blood-brain barrier permeability via reactivation of the hypoxia-angiogenesis program. J Immunol 177:5574-5584

7. Arnett HA, Mason J, Marino M, Suzuki K, Matsushima GK, Ting JP (2001) TNF alpha promotes proliferation of oligodendrocyte progenitors and remyelination. Nat Neurosci 4:1116-1122

8. Bakker DA, Ludwin SK (1987) Blood-brain barrier permeability during Cuprizone-induced demyelination. Implications for the pathogenesis of immune-mediated demyelinating diseases. J Neurol Sci 78:125-137

9. Balashov KE, Aung LL, Dhib-Jalbut S, Keller IA (2011) Acute multiple sclerosis lesion: conversion of restricted diffusion due to vasogenic edema. J Neuroimaging 21:202-204

10. Benardais K, Kotsiari A, Skuljec J, Koutsoudaki PN, Gudi V, Singh V, Vulinovic F, Skripuletz T, Stangel M (2013) Cuprizone [Bis(Cyclohexylidenehydrazide)] is selectively toxic for mature oligodendrocytes. Neurotox Res 24:244-250

11. Berghoff SA, Gerndt N, Winchenbach J, Stumpf SK, Hosang L, Odoardi F, Ruhwedel T, Bohler C, Barrette B, Stassart R, Liebetanz D, Dibaj P, Mobius W, Edgar JM, Saher G (2017) Dietary cholesterol promotes repair of demyelinated lesions in the adult brain. Nat Commun 8:1424

12. Boretius S, Escher A, Dallenga T, Wrzos C, Tammer R, Bruck W, Nessler S, Frahm J, Stadelmann C (2012) Assessment of lesion pathology in a new animal mode of MS by multiparametric MRI and DTI. Neurolmage 59:2678-2688

13. Buschmann JP, Berger $K$, Awad H, Clarner T, Beyer C, Kipp M (2012) Inflammatory response and chemokine expression in the white matter corpus callosum and gray matter cortex region during cuprizone-induced demyelination. J Mol Neurosci 48:66-76

14. Camire RB, Beaulac HJ, Willis CL (2015) Transitory loss of glia and the subsequent modulation in inflammatory cytokines/chemokines regulate paracellular claudin-5 expression in endothelial cells. J Neuroimmunol 284:57-66

15. Carlton WW (1969) Spongiform encephalopathy induced in rats and guinea pigs by cuprizone. Exp Mol Pathol 10:274-287

16. Clarner $T$, Janssen $K$, Nellessen $L$, Stangel M, Skripuletz $T$, Krauspe $B$, Hess FM, Denecke B, Beutner C, Linnartz-Gerlach B, Neumann H, Vallieres L, Amor S, Ohl K, Tenbrock K, Beyer C, Kipp M (2015) CXCL10 triggers early microglial activation in the cuprizone model. J Immunol 194:3400-3413
17. Cramer SP, Modvig S, Simonsen HJ, Frederiksen JL, Larsson HB (2015) Permeability of the blood-brain barrier predicts conversion from optic neuritis to multiple sclerosis. Brain 138:2571-2583

18. Cramer SP, Simonsen H, Frederiksen JL, Rostrup E, Larsson HB (2014) Abnormal blood-brain barrier permeability in normal appearing white matter in multiple sclerosis investigated by MRI. Neuroimage Clin 4:182-189

19. Davalos D, Baeten KM, Whitney MA, Mullins ES, Friedman B, Olson ES, Ryu JK, Smirnoff DS, Petersen MA, Bedard C, Degen JL, Tsien RY, Akassoglou K (2014) Early detection of thrombin activity in neuroinflammatory disease. Ann Neurol 75:303-308

20. Ferrari CC, Depino AM, Prada F, Muraro N, Campbell S, Podhajcer O, Perry $\mathrm{VH}$, Anthony DC, Pitossi FJ (2004) Reversible demyelination, blood-brain barrier breakdown, and pronounced neutrophil recruitment induced by chronic IL-1 expression in the brain. Am J Pathol 165:1827-1837

21. Geurts JJ, Barkhof F (2008) Grey matter pathology in multiple sclerosis. Lancet Neurol 7:841-851

22. Grabert K, Michoel T, Karavolos MH, Clohisey S, Baillie JK, Stevens MP, Freeman TC, Summers KM, McColl BW (2016) Microglial brain regiondependent diversity and selective regional sensitivities to aging. Nat Neurosci 19:504-516

23. Gudi V Moharregh-Khiabani D, Skripuletz T, Koutsoudaki PN, Kotsiari A, Skuljec J, Trebst C, Stangel M (2009) Regional differences between grey and white matter in cuprizone induced demyelination. Brain Res 1283:127-138

24. Hesse A, Wagner M, Held J, Bruck W, Salinas-Riester G, Hao Z, Waisman A Kuhlmann T (2010) In toxic demyelination oligodendroglial cell death occurs early and is FAS independent. Neurobiol Dis 37:362-369

25. Iqbal M, Ho HL, Petropoulos S, Moisiadis VG, Gibb W, Matthews SG (2012) Pro-inflammatory cytokine regulation of P-glycoprotein in the developing blood-brain barrier. PLoS One 7:e43022

26. Jung S, Aliberti J, Graemmel P, Sunshine MJ, Kreutzberg GW, Sher A, Littman DR (2000) Analysis of fractalkine receptor CX(3)CR1 function by targeted deletion and green fluorescent protein reporter gene insertion. Mol Cell Biol 20:4106-4114

27. Kang Z, Liu L, Spangler R, Spear C, Wang C, Gulen MF, Veenstra M, Ouyang W, Ransohoff RM, Li X (2012) IL-17-induced Act1-mediated signaling is critical for cuprizone-induced demyelination. J Neurosci 32:8284-8292

28. Keep RF, Hua Y, Xi G (2012) Brain water content. A misunderstood measurement? Transl Stroke Res 3:263-265

29. Klicek R, Kolenc D, Suran J, Drmic D, Brcic L, Aralica G, Sever M, Holjevac J, Radic B, Turudic T, Kokot A, Patrlj L, Rucman R, Seiwerth S, Sikiric P (2013) Stable gastric pentadecapeptide BPC 157 heals cysteamine-colitis and colon-colon-anastomosis and counteracts cuprizone brain injuries and motor disability. J Physiol Pharmacol 64:597-612

30. Kondo A, Nakano T, Suzuki K (1987) Blood-brain barrier permeability to horseradish peroxidase in twitcher and cuprizone-intoxicated mice. Brain Res 425:186-190

31. Kotter MR, Li WW, Zhao C, Franklin RJ (2006) Myelin impairs CNS remyelination by inhibiting oligodendrocyte precursor cell differentiation. J Neurosci 26:328-332

32. Krauthausen M, Saxe S, Zimmermann J, Emrich M, Heneka MT, Muller M (2014) CXCR3 modulates glial accumulation and activation in cuprizone-induced demyelination of the central nervous system. J Neuroinflammation 11:109

33. Lecuyer MA, Kebir H, Prat A (2016) Glial influences on BBB functions and molecular players in immune cell trafficking. Biochim Biophys Acta 1862:472-482

34. Li Y, Song Y, Zhao L, Gaidosh G, Laties AM, Wen R (2008) Direct labeling and visualization of blood vessels with lipophilic carbocyanine dye Dil. Nat Protoc 3:1703-1708

35. Liddelow SA, Guttenplan KA, Clarke LE, Bennett FC, Bohlen CJ, Schirmer L, Bennett ML, Munch AE, Chung WS, Peterson TC, Wilton DK, Frouin A, Napier BA, Panicker N, Kumar M, Buckwalter MS, Rowitch DH, Dawson VL, Dawson TM, Stevens B, Barres BA (2017) Neurotoxic reactive astrocytes are induced by activated microglia. Nature 541:481-487

36. Liu L, Belkadi A, Darnall L, Hu T, Drescher C, Cotleur AC, Padovani-Claudio D, He T, Choi K, Lane TE, Miller RH, Ransohoff RM (2010) CXCR2-positive neutrophils are essential for cuprizone-induced demyelination: relevance to multiple sclerosis. Nat Neurosci 13:319-326

37. Luissint AC, Artus C, Glacial F, Ganeshamoorthy K, Couraud PO (2012) Tight junctions at the blood brain barrier: physiological architecture and diseaseassociated dysregulation. Fluids Barriers CNS 9:23

38. Lundgaard I, Osorio MJ, Kress BT, Sanggaard S, Nedergaard M (2014) White matter astrocytes in health and disease. Neuroscience 276:161-173 
39. Mason JL, Suzuki K, Chaplin DD, Matsushima GK (2001) Interleukin-1 beta promotes repair of the CNS. J Neurosci 21:7046-7052

40. Mayerhofer R, Frohlich EE, Reichmann F, Farzi A, Kogelnik N, Frohlich E, Sattler W, Holzer P (2017) Diverse action of lipoteichoic acid and lipopolysaccharide on neuroinflammation, blood-brain barrier disruption, and anxiety in mice. Brain Behav Immun 60:174-187

41. Nyul-Toth A, Suciu M, Molnar J, Fazakas C, Hasko J, Herman H, Farkas AE, Kaszaki J, Hermenean A, Wilhelm I, Krizbai IA (2016) Differences in the molecular structure of the blood-brain barrier in the cerebral cortex and white matter: an in silico, in vitro, and ex vivo study. Am J Physiol Heart Circ Physiol 310:H1702-H1714

42. Paul D, Ge S, Lemire Y, Jellison ER, Serwanski DR, Ruddle NH, Pachter JS (2014) Cell-selective knockout and 3D confocal image analysis reveals separate roles for astrocyte-and endothelial-derived CCL2 in neuroinflammation. J Neuroinflammation 11:10

43. Paul R, Koedel U, Winkler F, Kieseier BC, Fontana A, Kopf M, Hartung HP, Pfister HW (2003) Lack of IL-6 augments inflammatory response but decreases vascular permeability in bacterial meningitis. Brain 126:1873-1882

44. Pfeiffer F, Schafer J, Lyck R, Makrides V, Brunner S, Schaeren-Wiemers N, Deutsch U, Engelhardt B (2011) Claudin-1 induced sealing of blood-brain barrier tight junctions ameliorates chronic experimental autoimmune encephalomyelitis. Acta Neuropathol 122:601-614

45. Praet J, Guglielmetti C, Berneman Z, Van der Linden A, Ponsaerts P (2014) Cellular and molecular neuropathology of the cuprizone mouse model: clinical relevance for multiple sclerosis. Neurosci Biobehav Rev 47:485-505

46. Probert L, Akassoglou K, Pasparakis M, Kontogeorgos G, Kollias G (1995) Spontaneous inflammatory demyelinating disease in transgenic mice showing central nervous system-specific expression of tumor necrosis factor alpha. Proc Natl Acad Sci U S A 92:11294-11298

47. Rochfort KD, Collins LE, Murphy RP, Cummins PM (2014) Downregulation of blood-brain barrier phenotype by proinflammatory cytokines involves NADPH oxidase-dependent ROS generation: consequences for interendothelial adherens and tight junctions. PLoS One 9:e101815

48. Roemer SF, Parisi JE, Lennon VA, Benarroch EE, Lassmann $\mathrm{H}$, Bruck W, Mandler RN, Weinshenker BG, Pittock SJ, Wingerchuk DM, Lucchinetti CF (2007) Pattern-specific loss of aquaporin-4 immunoreactivity distinguishes neuromyelitis optica from multiple sclerosis. Brain 130:1194-1205

49. Saher G, Rudolphi F, Corthals K, Ruhwedel T, Schmidt KF, Löwel S, Dibaj P, Barrette B, Möbius W, Nave KA (2012) Therapy of Pelizaeus-Merzbacher disease in mice by feeding a cholesterol-enriched diet. Nat Med 18:1130-1135

50. Satoh J, Tabunoki H, Yamamura T, Arima K, Konno H (2007) Human astrocytes express aquaporin-1 and aquaporin-4 in vitro and in vivo. Neuropathology: official journal of the Japanese society of. Neuropathology 27:245-256

51. Schellenberg AE, Buist R, Yong WW, Del Bigio MR, Peeling J (2007) Magnetic resonance imaging of blood-spinal cord barrier disruption in mice with experimental autoimmune encephalomyelitis. Magn Reson Med 58:298-305

52. Skripuletz T, Hackstette D, Bauer K, Gudi V, Pul R, Voss E, Berger K, Kipp M, Baumgartner W, Stangel M (2013) Astrocytes regulate myelin clearance through recruitment of microglia during cuprizone-induced demyelination. Brain 136:147-167

53. Stamatovic SM, Keep RF, Kunkel SL, Andjelkovic AV (2003) Potential role of MCP-1 in endothelial cell tight junction 'opening': signaling via rho and rho kinase. J Cell Sci 116:4615-4628

54. Stokum JA, Gerzanich V, Simard JM (2016) Molecular pathophysiology of cerebral edema. J Cereb Blood Flow Metab 36:513-538

55. Tamashiro TT, Dalgard CL, Byrnes KR (2012) Primary microglia isolation from mixed glial cell cultures of neonatal rat brain tissue. J Vis Exp:e3814

56. van Horssen J, Brink BP, de Vries HE, van der Valk P, Bo L (2007) The bloodbrain barrier in cortical multiple sclerosis lesions. J Neuropathol Exp Neurol 66:321-328

57. Voss EV, Skuljec J, Gudi V, Skripuletz T, Pul R, Trebst C, Stangel M (2012) Characterisation of microglia during de- and remyelination: can they create a repair promoting environment? Neurobiol Dis 45:519-528

58. Wang Y, Jin S, Sonobe Y, Cheng Y, Horiuchi H, Parajuli B, Kawanokuchi J, Mizuno T, Takeuchi H, Suzumura A (2014) Interleukin-1beta induces bloodbrain barrier disruption by downregulating sonic hedgehog in astrocytes. PLoS One 9:e110024
59. Werring DJ, Brassat D, Droogan AG, Clark CA, Symms MR, Barker GJ, MacManus DG, Thompson AJ, Miller DH (2000) The pathogenesis of lesions and normal-appearing white matter changes in multiple sclerosis: a serial diffusion MRI study. Brain 123(Pt 8:1667-1676

60. Wolburg-Buchholz K, Mack AF, Steiner E, Pfeiffer F, Engelhardt B, Wolburg H (2009) Loss of astrocyte polarity marks blood-brain barrier impairment during experimental autoimmune encephalomyelitis. Acta Neuropathol 118:219-233
Submit your next manuscript to BioMed Central and we will help you at every step:

- We accept pre-submission inquiries

- Our selector tool helps you to find the most relevant journal

- We provide round the clock customer support

- Convenient online submission

- Thorough peer review

- Inclusion in PubMed and all major indexing services

- Maximum visibility for your research

Submit your manuscript at www.biomedcentral.com/submit
C Biomed Central 
a

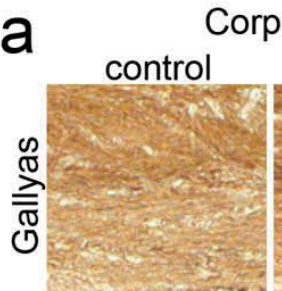

Corpus callosum (CC)
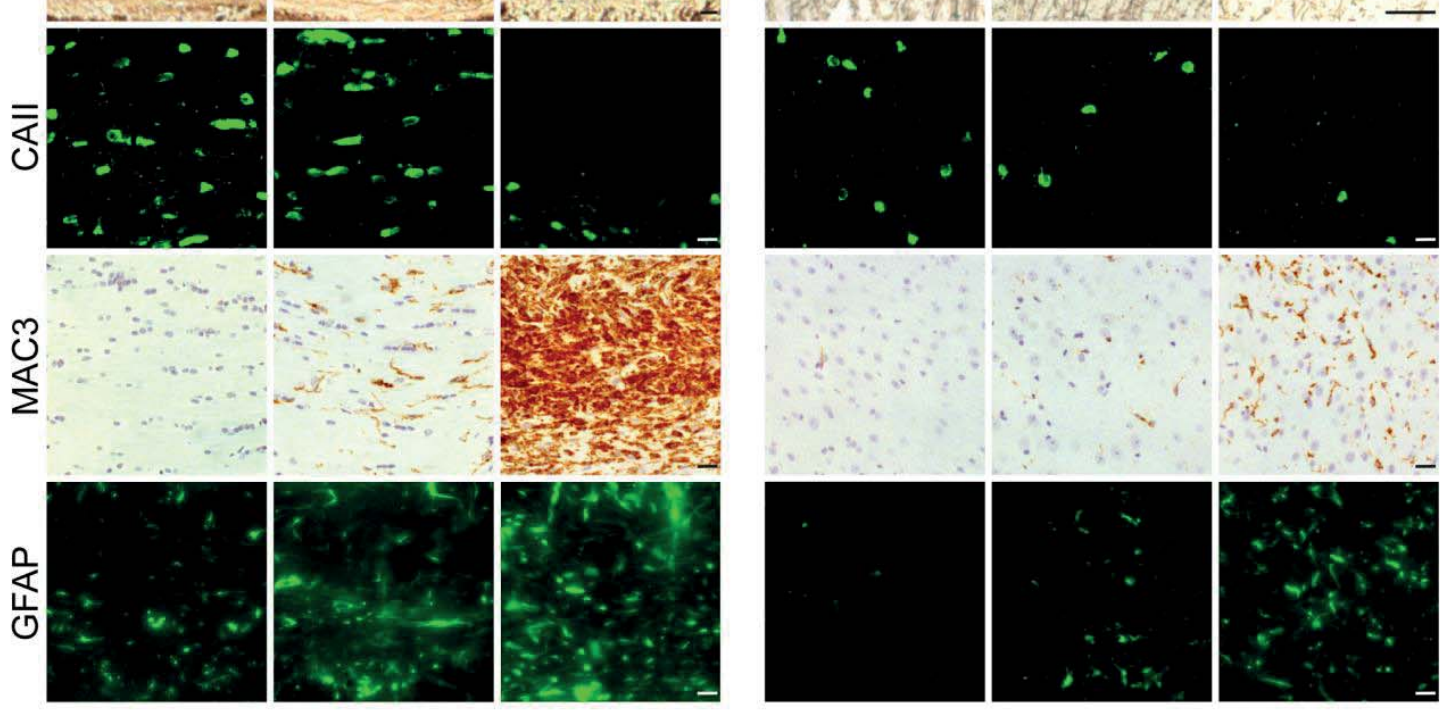

b
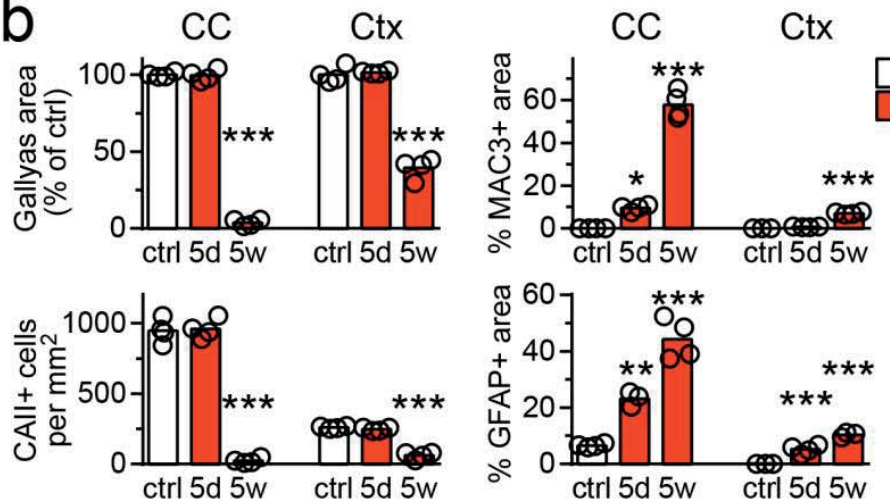

Figure S1. Time course of cuprizone induced pathology in corpus callosum and cortex.

(a) Representative pictures of the corpus callosum (CC) and the cortex (Ctx) of untreated control mice and mice after cuprizone exposure for 5 days and 5 weeks assessing myelination (Gallyas silver impregnation), mature oligodendrocytes (CAII), activated microglia (MAC3), and astrocytes (GFAP) (Scale bars: $20 \mu \mathrm{m})$ with quantification in (b). Each bar represents the mean value of $\mathrm{N}=3-4$ animals per condition with individual data points. Significance to control was evaluated by 1way ANOVA with Tukey's post test ( ${ }^{*} \mathrm{P}<$ $\left.0.05,{ }^{* *} \mathrm{P}<0.01,{ }^{* * *} \mathrm{P}<0.001\right)$. 
a
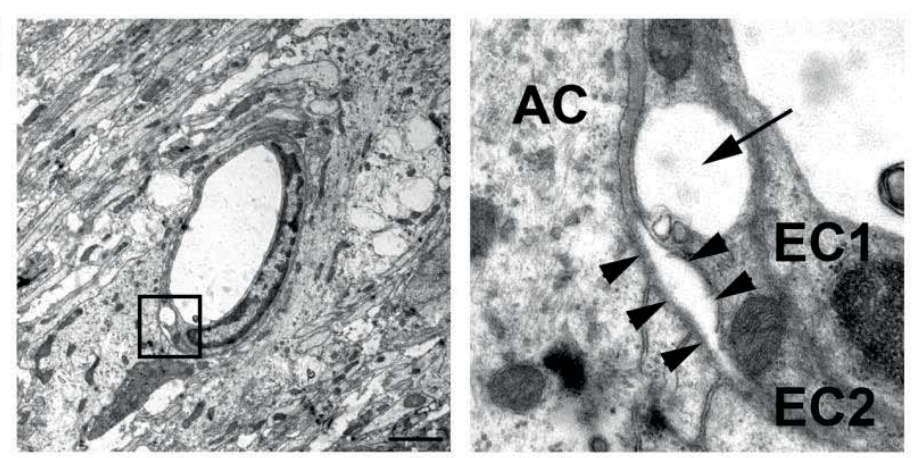

b
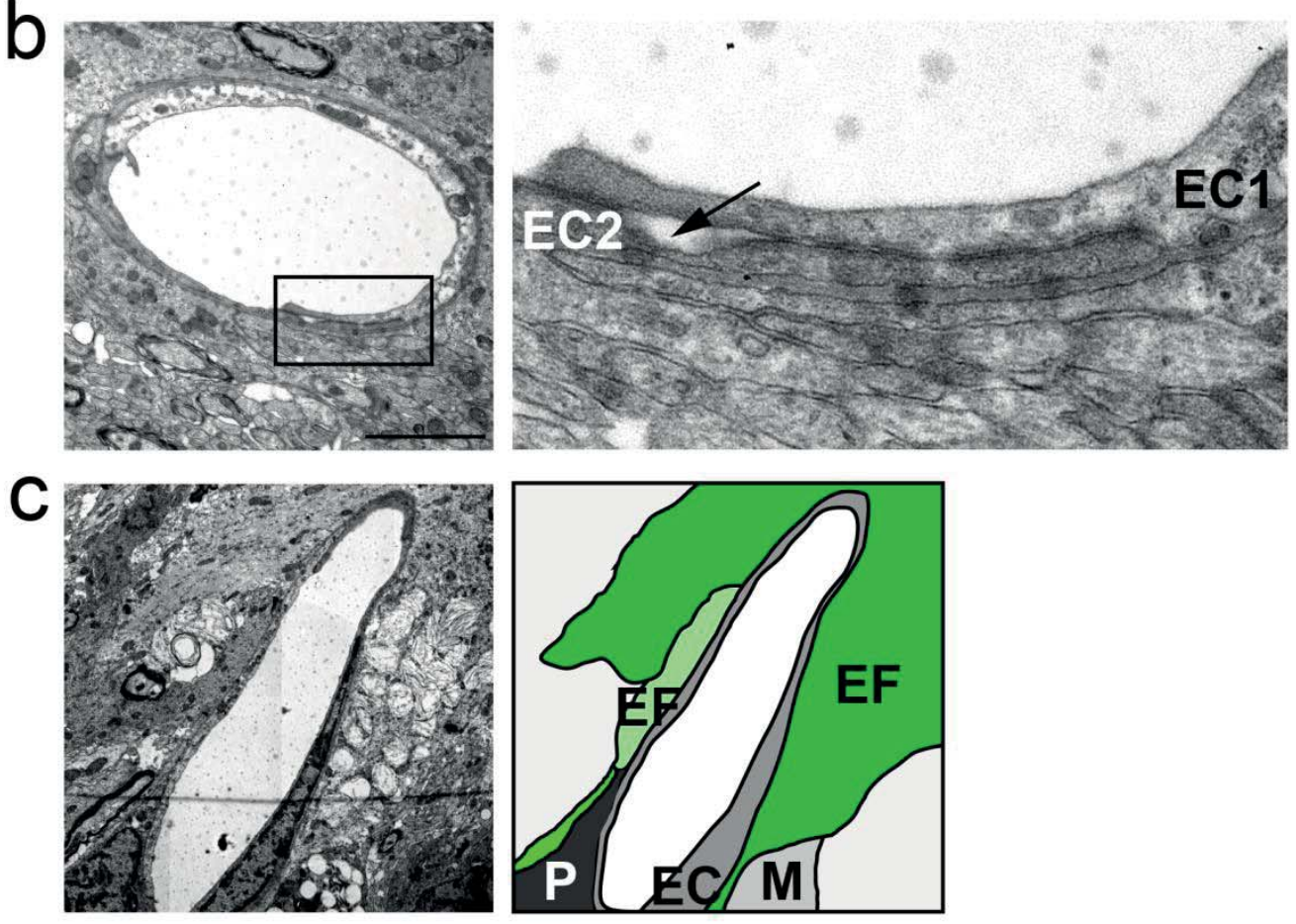

Figure S2. Endthelial junctions in mice after 5 weeks cuprizone.

Electron microscopic images of capillaries showing disconnected endothelial and astroglial basement membranes (a, arrowheads), an affected endothelial cell (b) with electron light cytoplasm and focal disruption of endothelial tight junctions ( $a, b$, arrow). Astroglial endfeet often appeared swollen (c, green). Scale bars: $2 \mu \mathrm{m}$ (AC, astrocyte; EC, endothelial cell; EF, astroglial endfoot; M, microglia/macrophage; $P$, pericyte). 

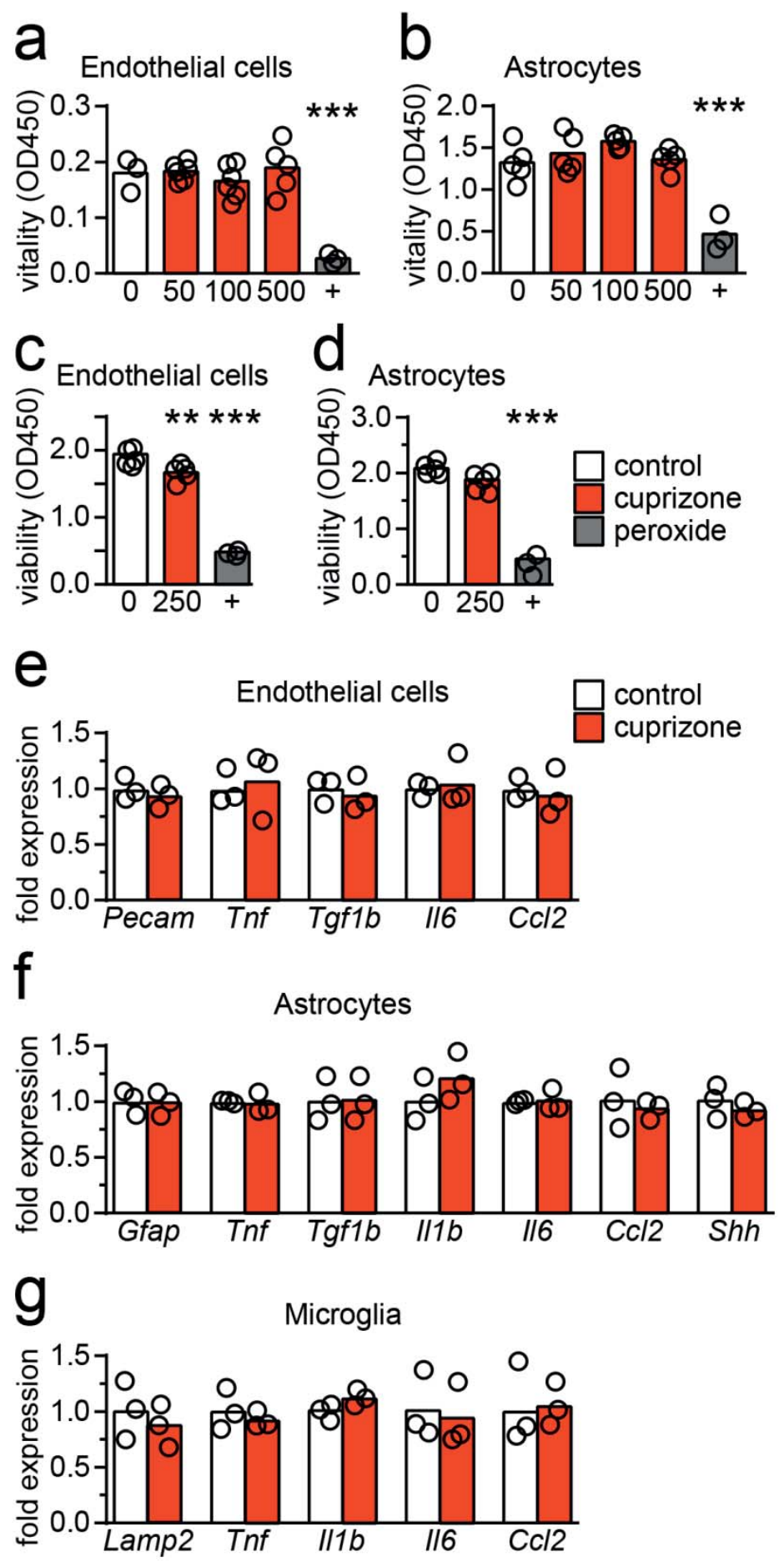

Figure S3: Cuprizone affects endothelial cells but not astrocytes in vitro. Cell vitality measurements (WST1 assays) of primary $(a, c)$ endothelial cells or $(b, d)$ astrocytes after exposure to vehicle $(0$, white) or to increasing concentrations of cuprizone $(50-500 \mu \mathrm{M}$, red) for $24 \mathrm{~h}(\mathrm{a}, \mathrm{b})$ or with $250 \mu \mathrm{M}$ cuprizone for $72 \mathrm{~h}(\mathrm{c}, \mathrm{d})(\mathrm{N}=5$ per condition). Incubation with $20 \mu \mathrm{M}$ peroxide for $4 \mathrm{~h}$ induced cell death and was used as positive control $(\mathrm{N}=3,+)$.

Significance to vehicle was evaluated by $(a, b)$ 1way ANOVA with Dunnett's post test or (c, d) Student's t-test $\left({ }^{* *} P<0.01\right.$, $\left.{ }^{* * *} P<0.001\right)$. (e, f, g)

Quantitative RT-PCR analysis on cultured primary endothelial cells (e), astrocytes (f), and microglia (g) challenged with $250 \mu \mathrm{M}$ cuprizone for $48 \mathrm{~h}$. Results show mean fold change with individual data points of $\mathrm{N}=3$ cultures normalized to vehicle control (set to 1 ). 

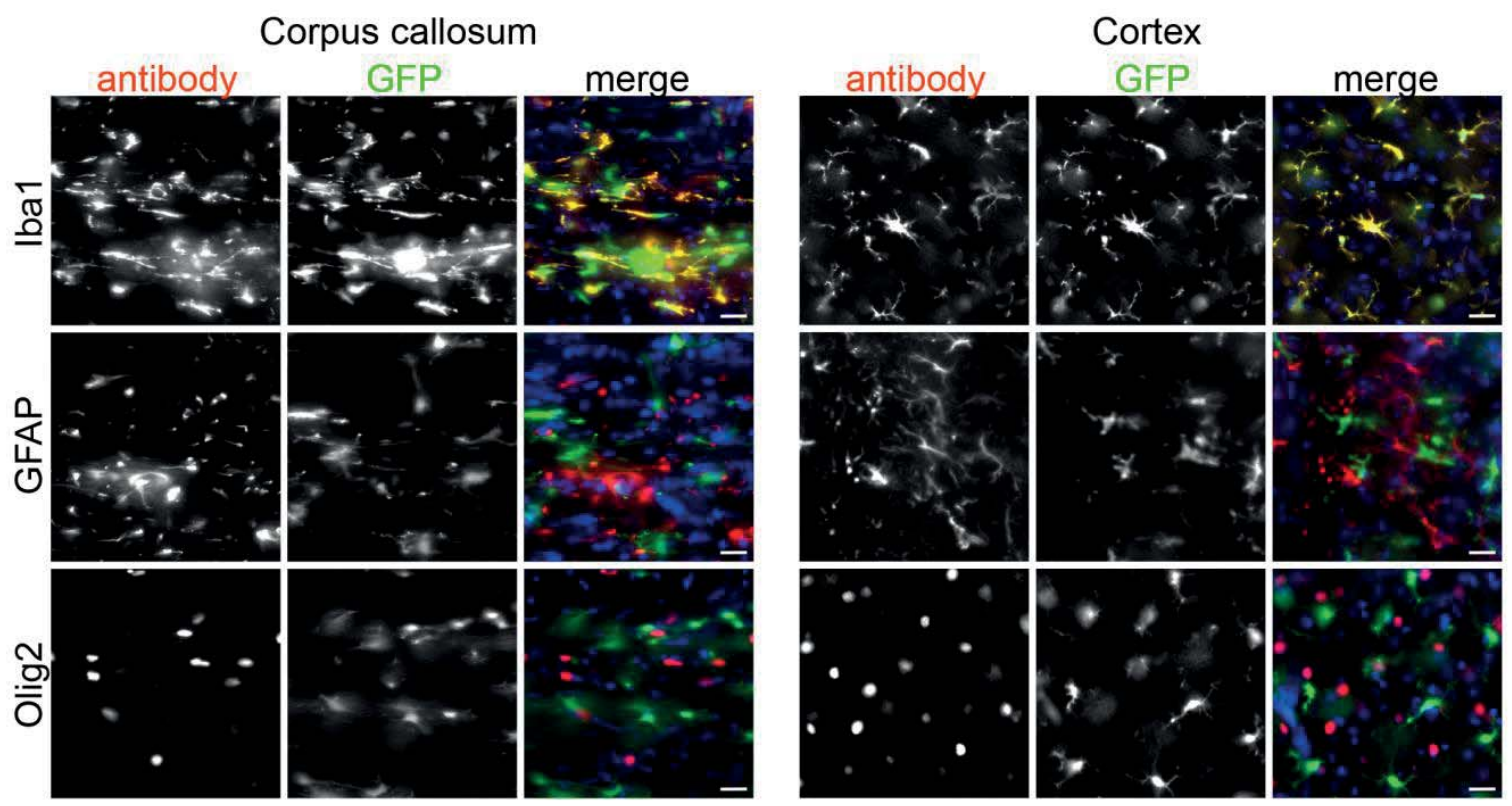

Figure S4. CXCR3 is expressed by microglia in cuprizone fed mice.

Direct GFP fluorescence of CXCR3 3 GP/GFP mice together with immunolabeling of cell type specific markers for microglia (Iba1), astrocytes (GFAP), or oligodendroglia (Olig2) in the corpus callosum and cortex in CXCR3 deficient mice that had been exposed to cuprizone for five days (Scale bars: $20 \mu \mathrm{m}$ ). 


\subsection{Manuscript:}

\section{Phase and cell-type specific post-squalene sterol synthesis coordinates inflammation and remyelination}

Stefan A. Berghoff ${ }^{1}$, Lena Spieth ${ }^{1}$, Ting Sun ${ }^{1,6}$, Lennart Schlaphoff ${ }^{1}$, Leon Hosang ${ }^{2}$, Constanze Depp ${ }^{1}$, Tim Düking ${ }^{1}$, Jan Winchenbach ${ }^{1}$, Jonathan Neuber ${ }^{1}$, David Ewers $^{1}$, Patricia Scholz ${ }^{3}$, Franziska Paap ${ }^{4}$, Ludovico Cantuti-Castelvetri ${ }^{7}$, Andrew O. Sasmita ${ }^{1}$, Martin Meschkat ${ }^{1}$, Torben Ruhwedel ${ }^{1}$, Wiebke Möbius ${ }^{1}$, Francesca Odoardi ${ }^{2}$, Till Ischebeck ${ }^{3}$, Julia M. Edgar ${ }^{5}$, Marco Prinz ${ }^{8}$, Roman Sankowski ${ }^{8}$ Christine Stadelmann-Nessler ${ }^{4}$, Mikael Simons ${ }^{7}$, Klaus-Armin Nave ${ }^{1}$, and Gesine Saher ${ }^{1 *}$

${ }^{1}$ Department of Neurogenetics, Max Planck Institute of Experimental Medicine, Hermann-Rein-Str. 3, 37075 Göttingen, Germany.

${ }^{2}$ Institute of Neuroimmunology and Multiple Sclerosis Research, University Medical Centre Göttingen, Von-Siebold-Str. 3a, 37075 Göttingen, Germany.

${ }^{3}$ Albrecht-von-Haller-Institute for Plant Sciences, Dept. of Plant Biochemistry, GeorgAugust-University Göttingen, Justus-von-Liebig-Weg 11, 37073 Göttingen, Germany.

${ }^{4}$ Institute for Neuropathology, University Medical Centre Göttingen , Robert-Koch-Str. 40, 37075 Göttingen

${ }^{5}$ Applied Neurobiology Group, Institute of Infection, Immunity and Inflammation, College of Medical Veterinary and Life Sciences, University of Glasgow, Glasgow G12-8TA, UK.

${ }^{6}$ Institute for Medical Systembiology, Center for Molecular Neurobiology Hamburg, Martinistr. 52, 20246 Hamburg

${ }^{7}$ German Center for Neurodegenerative Diseases, Molecular Neurobiology , FeodorLynen-Str. 17, 81377 München

${ }^{8}$ Institute of Neuropathology, Medical Faculty, University of Freiburg, Freiburg, Germany 


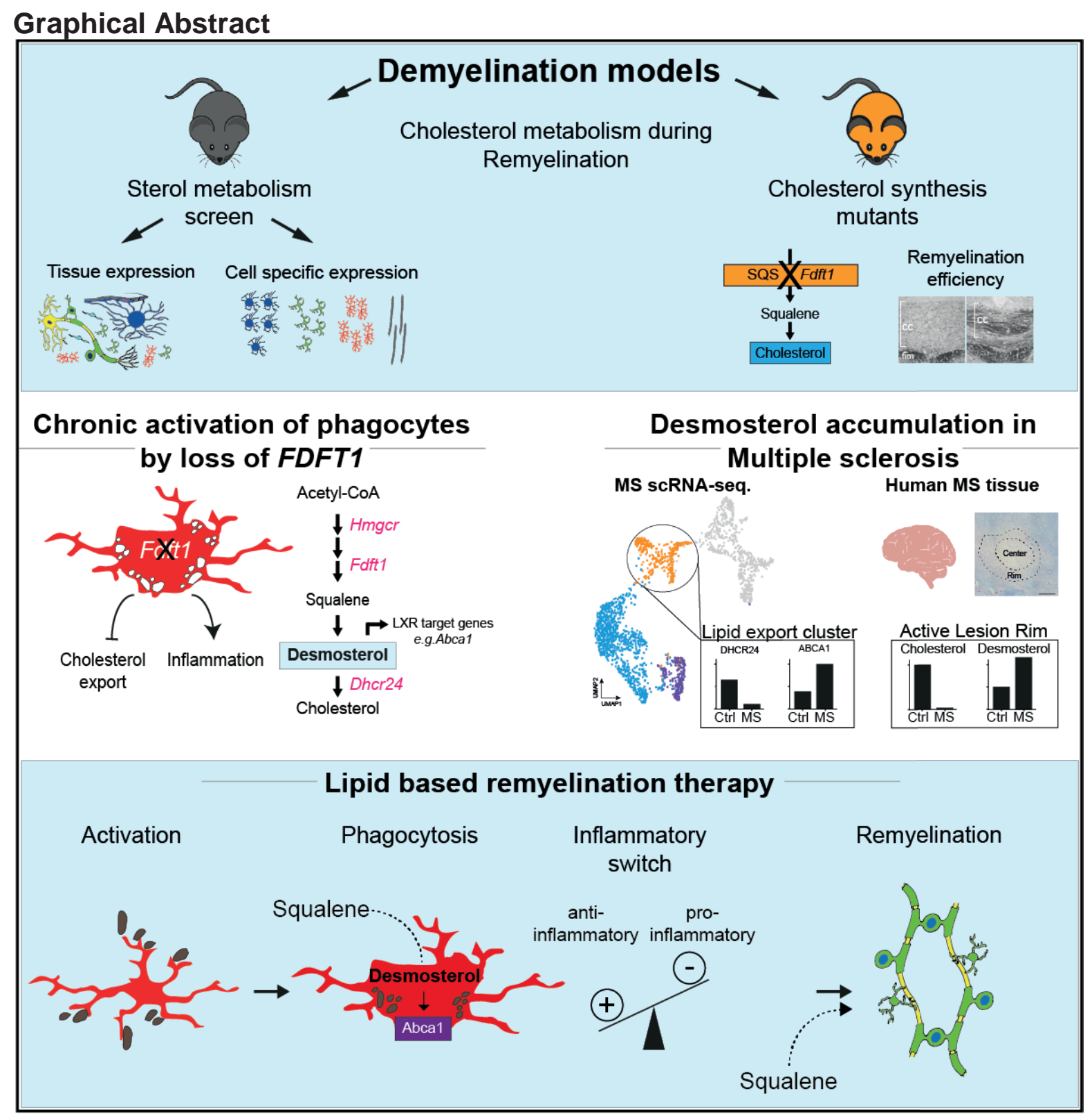

\section{Highlights}

- Profiling of cell type specific cholesterol metabolism during remyelination

- Phagocytes accumulate the LXR ligand desmosterol following myelin uptake

- Desmosterol accumulates in phagocytes of Multiple Sclerosis lesions

- Squalene therapy reduces inflammation by boosting desmosterol-LXR signaling 


\section{Phase and cell-type specific post-squalene sterol synthesis coordinates inflammation and remyelination}

Stefan A. Berghoff ${ }^{1}$, Lena Spieth ${ }^{1}$, Ting Sun ${ }^{1,6}$, Lennart Schlaphoff ${ }^{1}$, Leon Hosang ${ }^{2}$,Constanze Depp ${ }^{1}$, Tim Düking ${ }^{1}$, Jan Winchenbach ${ }^{1}$, Jonathan Neuber ${ }^{1}$, David Ewers ${ }^{1}$, Patricia Scholz ${ }^{3}$, Franziska Paap ${ }^{4}$, Ludovico Cantuti-Castelvetri ${ }^{7}$, Andrew O. Sasmita, Martin Meschkat ${ }^{1}$, Torben Ruhwedel ${ }^{1}$, Wiebke Möbius ${ }^{1}$, Francesca Odoardi ${ }^{2}$, Till Ischebeck ${ }^{3}$, Julia M. Edgar ${ }^{5}$, Marco Prinz ${ }^{8}$, Roman Sankowski ${ }^{8}$ Christine Stadelmann-Nessler ${ }^{4}$,Mikael Simons ${ }^{7}$, Klaus-Armin Nave ${ }^{1}$, and Gesine Saher ${ }^{1 *}$

${ }^{1}$ Department of Neurogenetics, Max Planck Institute of Experimental Medicine, Hermann-Rein-Str. 3, 37075 Göttingen, Germany.

${ }^{2}$ Institute of Neuroimmunology and Multiple Sclerosis Research, University Medical Centre Göttingen, Von-Siebold-Str. 3a, 37075 Göttingen, Germany.

${ }^{3}$ Albrecht-von-Haller-Institute for Plant Sciences, Dept. of Plant Biochemistry, Georg-AugustUniversity Göttingen, Justus-von-Liebig-Weg 11, 37073 Göttingen, Germany.

${ }^{4}$ Institute for Neuropathology, University Medical Centre Göttingen , Robert-Koch-Str. 40, 37075 Göttingen

\footnotetext{
${ }^{5}$ Applied Neurobiology Group, Institute of Infection, Immunity and Inflammation, College of Medical Veterinary and Life Sciences, University of Glasgow, Glasgow G12-8TA, UK.

${ }^{6}$ Institute for Medical Systembiology, Center for Molecular Neurobiology Hamburg, Martinistr. 52, 20246 Hamburg

${ }^{7}$ German Center for Neurodegenerative Diseases, Molecular Neurobiology , Feodor-Lynen-Str. 17, 81377 München

${ }^{8}$ Institute of Neuropathology, Medical Faculty, University of Freiburg, Freiburg, Germany

*Correspondence: saher@em.mpg.de
}

\section{Summary}

The primary origin of brain cholesterol is de novo synthesis and most if not all brain cells contribute to central nervous system (CNS) cholesterol homeostasis. In demyelinating diseases such as multiple sclerosis (MS), brain cholesterol homeostasis is disturbed, likely contributing to pathology. To investigate the relationship between cholesterol synthesis, remyelination and inflammation, we integrated cell specific and tissue based sterol gene expression profiles with remyelination efficiency of cell type specific conditional cholesterol synthesis mutants. We identified a conserved pathway leading to the accumulation of the LXR ligand desmosterol in phagocytes following myelin uptake that limits inflammation. This pathway can be boosted by administration of the natural cholesterol precursor squalene which reduces inflammatory response in phagocytes and also directly stimulates myelination by oligodendroglia. Importantly, we provide evidence of desmosterol accumulation in phagocytes in MS tissue. Therefore we propose squalene administration as potential therapeutic approach in MS. 


\section{Introduction}

Brain cholesterol metabolism is tightly regulated between different CNS cells to maintain cholesterol homeostasis (Dietschy, 2009; Saher and Stumpf, 2015). In demyelinating disease such as Multiple Sclerosis (MS) brain lipid metabolism is disturbed (Nogueras et al., 2019; Vuletic et al., 2014; Wilson and Tocher, 1991), likely contributing to pathology. Some genetic variants that are linked to disease progression associate with cholesterol metabolism in neurodegenerative disorders. Among MS patients, variants of APOE involved in cholesterol efflux/transport (Pinholt et al., 2006), mutations of NR1H3(LXRß) controlling transcriptional regulation of cholesterol homeostasis (Wang et al., 2016) and variants on chr8p23 near FDFT1 crucial for cholesterol synthesis (Smets et al., 2018) have been connected to disease expression. Previously, we could show that peripheral cholesterol supplementation in mouse models of adult demyelination supports remyelination by directly targeting oligodendroglia (Berghoff et al., 2017b). However, therapeutic management of MS has largely focused on immunomodulatory treatments, thereby targeting inflammation (Rodgers et al., 2013). Nonetheless, strategies enhancing remyelination in particular in combination therapy are needed to effectively restore neurological disabilities (Franklin and Ffrench-Constant, 2008). Therapeutic factors specifically modulating lipid metabolism have recently gain attention in remyelination therapy. This might be highlighted by recent studies that suggest targeting of the cholesterol synthesis pathways as therapeutic objective during remyelination (Itoh et al., 2018; Voskuhl et al., 2019). However, the contribution of individual cell types to cholesterol metabolism during demyelination still remains poorly understood. Thus, we comprehensively analyzed cholesterol metabolism during remyelination by cell type specific and tissue based sterol gene expression profiling and evaluated remyelination efficiency of cell type specific conditional cholesterol synthesis mutants during acute and chronic remyelination. Here we show that $(\mathrm{I})$ chronic remyelination is driven by the capability of oligodendroglia to synthesize cholesterol, (II) cholesterol synthesis ablation in phagocytes prevents accumulation of the LXR ligand desmosterol leading to chronic activation and lipid export deficiency thereby preventing remyelination, (III) exogenous squalene therapy reduces disease severity by inducing LXR activation through regulated accumulation of desmosterol suppressing inflammation, in addition to directly supporting myelination in oligodendroglia and (IV) squalene administration can be integrated in combination therapy suggesting implications for therapeutic intervention in MS.

\section{Results}

Cell and phase specific requirement of cholesterol synthesis during remyelination: Critical role of CNS phagocytes

To specifically determine brain cholesterol metabolism in demyelinating disease, cuprizone induced demyelination serves as an optimal experimental model system due to its 1.non-inflammatory etiology 2.repeating pathologic progression (de- and remyelination) and 3.orchestrated spatiotemporal predictability (Ransohoff, 2012). Following cuprizone induced oligodendrocyte depletion, acute remyelination occurs, that is characterized by extensive OPC proliferation, differentiation and myelination. In contrast, during chronic phase remyelination is limited, indicated by myelin visualization (Figure1A,1B) and expression of oligodendrocyte marker genes (Figure S1A)(Berghoff et al., 2017b; Matsushima and Morell, 2001). In order to characterize sterol metabolism during remyelination, 
we integrated cell specific and tissue based sterol gene expression profiles with remyelination efficiency of cell type specific conditional cholesterol synthesis mutants during acute and chronic phase (Figure1C). To evaluate cell type specific gene expression, established Figure1D) and validated (Figure1E,S1B) acute cell isolation protocols were applied (Berghoff et al., 2017a; Zhang et al., 2014). In parallel, conditional cholesterol synthesis mutants were generated to target CNS glia (Figure S1C,S2C). Efficient gene targeting of up to $97 \pm 0.7 \%$ did not induce prominent peripheral effects, evaluated by bodyweight and peripheral serum cholesterol (FigureS1C,S1D,S2C). To ensure cell type specific targeting during demyelination (FigureS1E), Tdto reporter expression was analyzed by cell marker co-labeling (FigureS1F). In the adult CNS, astrocytes show high transcript levels of cholesterol synthesis genes (FigureS1E, S1B) (Pfrieger and Ungerer, 2011; Zhang et al., 2014). In agreement with previous studies (Itoh et al., 2018), cholesterol synthesis genes in astrocytes were strongly reduced not only in cuprizone induced pathology (Figure $1 \mathrm{G}$ ) but also during inflammatory and genetic demyelination (Figure S2A,S2B). This was supported by comparable remyelination efficiency of astrocyte specific cholesterol mutants and controls (Figure1B,S2D), suggesting no contribution of astrocytic cholesterol during remyelination. Cholesterol availability is a prerequisite for myelination (Jurevics and Morell, 1995; Saher et al., 2005). To our surprise, we did not observe upregulation of genes related to cholesterol synthesis $(\mathrm{Hmgcr}$, Fdft1, Dhcr24, Cyp51) in corpus callosum samples isolated during acute remyelination, while expression levels increased during chronic remyelination (Figure1F). By analyzing the cell type specific expression of cholesterol synthesis genes, increased expression could be related to oligodendrocyte lineage cells (Figure $1 \mathrm{G}$ ), which has been recently suggested as potential therapeutic target (Voskuhl et al., 2019). Further supporting the essential needs of functional cholesterol synthesis in oligodendrocyte linage cells during chronic remyelination, oligodendrocyte differentiation, remyelination and behavioral performance was affected by targeting SQS/Fdft1 (Figure1H,S2E). In line, we recently showed that exogenous cholesterol promotes remyelination only during chronic remyelination (Berghoff et al., 2017b). Together, this raises the possibility of efficient remyelination after acute demyelination independent of cholesterol synthesis. However, as shown in Figure1G, cholesterol synthesis genes in isolated microglia during acute remyelination were partially increased (Hmgcr, Cyp51) in addition to genes related to cholesterol uptake (Ldlr, Vldlr), storage (Soat1, Nceh1), export (Abca1, Abcg1, and Apoe) and phagocytosis (CD36). To our surprise, cholesterol synthesis ablation in microglia leads to severely impaired remyelination indicating functional consequence of cholesterol synthesis gene regulation in these cells (Figure $1 \mathrm{H}$ ). Hence, we conclude that chronic remyelination seem to be driven by the capability of oligodendroglia to synthesize cholesterol, while loss of post squalene cholesterol synthesis in microglia impacts on remyelination efficiency after acute demyelination.

\section{Cholesterol synthesis deficiency in CNS phagocytes induces chronic activation and lipid export deficiency}

In mammalian cells, cholesterol synthesis and export is tightly regulated. In demyelinating disease, cholesterol export deficiency by specifically targeting the cholesterol transporters ABCA1 and ABCG1 in phagocytes (FigureS3A) has been linked to limited remyelination (Cantuti-Castelvetri et al., 2018). Because remyelination efficiency following demyelination was impaired by interfering with cholesterol 
synthesis in microglia (SQScKO), we examined histopathologic hallmarks in contrast to pathology in microglial cholesterol export deficient animals (ABC1CKO) (Figure2A). As expected, we detected a $\sim 25 \%$ reduction of remyelination in the corpus callosum of $A B C 1 \mathrm{cKO}$ animals in contrast to a robust reduction of $\sim 60 \%$ of Gallyas positive myelinated fibers in SQScKO mice (Figure2B, S3B). Surprisingly, while in both mutants increased numbers of oligodendrocyte lineage cells compared to control were apparent, we detected a significant increase of CAll positive differentiating oligodendrocytes only in the ABC1cKO corpus callosum, which was also detected by transcriptional analysis (Figure2 $\mathrm{B}, 2 \mathrm{C}$ ).OPC proliferation and differentiation is influenced by the local inflammatory environment (Alizadeh and Karimi-Abdolrezaee, 2016; Franklin, 2002). By analyzing several inflammatory mediator genes in tissue samples, we found decreased expression of pro-inflammatory genes (Nos2, I112) and increased expression of anti-inflammatory/pro-differentiating genes (Arg1, IL10) in ABC1cKO animals (Figure2C). In contrast, in corpus callosum of microglial SQScKO animals, tissue environment exhibit a proinflammatory gene signature, likely explaining inhibition of oligodendrocyte differentiation.

Myelin phagocytosis and lipid loading induced phenotypic switching of proinflammatory phagocytes, thereby suppressing inflammation (Boven et al., 2006; Grajchen et al., 2018). Hence, proinflammatory gene signature in SQScKO phagocytes and impaired remyelination could result as a consequence of impaired phagocytosis (Lampron et al., 2015; Loike et al., 2004). To examine this, isolated SQScKO bone marrow derived phagocytes (BMDM) were challenged with myelin in vitro (Figure2D). Effective gene targeting of SQS/Fdft1 in vitro was verified by staining for intracellular cholesterol under lipid depleted conditions and expression analysis of SQS/Fdft1, revealing a transcriptional reduction of $\sim 88 \%$ to control (FigureS3C,S3D). Phagocytosis of fluorescent labeled myelin was not impaired in SQScKO phagocytes even under pro-inflammatory conditions (Figure2D, S3E). In accordance, we did not observe significant difference in number of oligodendrocyte, oligodendrocyte linage cells, microgliosis and myelin loss during demyelination, indicating no interference with myelin uptake (FigureS3F,S3G). Accumulation of cholesterol in ABC1cKO phagocytes results in foam cell formation and appearance of cholesterol crystals (Cantuti-Castelvetri et al., 2018). Intriguingly, distinct phagocyte morphology observed in ABC1cKO tissue could also be detected in microglial cholesterol synthesis mutants (Figure2E). Conversely, we observed reduced expression (Figure2C) and protein abundance (Figure2G) of ABCA1, as well as appearance of foamy phagocytes and cholesterol crystals in microglial SQScKO tissue samples (Figure2E,2F). Cholesterol overload in $A B C 1$ cKO phagocytes has been linked to activation of the NLRP3 inflammasome and pyroptotic cell death (Duewell et al., 2010). Consistent with this, isolated CD11b+ phagocytes from demyelinated tissue of SQScKO animals expressed significantly increased transcript levels of genes associated with NLRP3 inflammasome activation (Figure2H). To investigate induced cell death by lipid overloading, isolated BMDMs of cholesterol synthesis and export mutants were chronically challenged with myelin (Figure2I,S3H). Substantiating our in vivo finding, SQScKO phagocytes showed decreased survival of up to $\sim 53 \%$ compared to only $\sim 19 \%$ survival of primary export deficient cells, with morphology attributed to pyroptotic cell death (Video S1). Hence, we conclude that cholesterol synthesis ablation in phagocytes results in partial cholesterol export deficiency in similarity to primary export deficient phagocytes. However, in contrast to export deficient phagocytes, cholesterol synthesis ablation 
additionally leads to chronic activation with proinflammatory gene signature thereby inhibiting oligodendrocyte differentiation and remyelination causing a distinct pathologic environment (FigureS9).

Of note, cuprizone (copper chelator) induced cell death has been selectively linked to mature oligodendrocytes (Benardais et al., 2013). However, copper plays an important role in the physiology of the innate immune system including phagocytes (Rossi-George et al., 2012; Stafford et al., 2013). Therefore we ask the question, whether pathology induced by cholesterol synthesis ablation in phagocytes is specific to the cuprizone model. We thus analyzed disease expression in phagocyte SQScKO animals following lysolecithin induced focal demyelination and inflammatory demyelinating pathology in EAE (Lassmann and Bradl, 2017). Excluding a specific effect related to cuprizone, we detected persistent microgliosis in spinal cord tissue following lysolecithin injection (MAC3 and IBA1 positive cells) (Figure $2 \mathrm{~J}, \mathrm{~S} 3 \mathrm{I}$ ) and inflammatory demyelination (CD11b+CD45 ${ }^{\text {low }}$ isolated cells) (Figure $2 \mathrm{~K}$ ), together with exacerbated disease following cholesterol synthesis ablation in microglia (Figure2K,S3J,S3K).

\section{Squalene administration rescues cholesterol export deficiency and proinflammatory gene signature in phagocytes}

Increased cholesterol availability does not impact on disease during inflammatory demyelination (Berghoff et al., 2017b; Boven et al., 2006). However, by genetic interference at the level of SQS/Fdft1 which is the first committed step to cholesterol, we identified intact post squalene cholesterol synthesis in phagocytes of pathological relevance during inflammatory disease. To specifically determine if loss of squalene and post squalene cholesterol synthesis accounts for exacerbated pathology (Figure $3 \mathrm{~A}$ ), we tested squalene supplementation during inflammatory demyelination that is driven by cells of the adaptive and innate immune system (e.g. phagocytes). To do so, we first applied squalene supplementation in microglial SQScKO animals given in a prophylactic treatment paradigm (Figure $3 B$ ). As shown in figure Figure $3 B$ and $3 C$, exogenous squalene reduced disease severity and inflammatory cell number in microglia SQScKO animals even further than expected, indicating additional impact on not CX3CR1-CreERT ${ }^{2}$ genetically targeted peripheral myeloid cells, due to the fast turnover and replenishment of bone marrow precursors (Kim et al., 2011; Parkhurst et al., 2013). To account for that, wildtype animals received exogenous squalene prophylactically. Notably, animals showed reduced clinical disease severity at peak of disease (FigureS4A) and during chronic phase (Figure3D) together with reduced number of inflammatory cells within CNS tissue (FigureS4B). Reduced number of inflammatory cells in squalene treated animals may be a result of decreased peripheral inflammatory stimulation or a reduction of recruitment to the CNS by amelioration of the inflammatory milieu within tissue (Lodygin et al., 2013; Pierson et al., 2012; Rezai-Zadeh et al., 2009). However, when we analyzed inflammatory cells in blood samples of squalene treated animals, we did not identify significant differences, indicating no impact of squalene one peripheral $\mathrm{T}$ cell and myeloid cell numbers (FigureS4C). Supporting reduced recruitment following squalene administration, SQS/Fdft1 ablation specifically in microglia results in increased number of phagocytes (CD11b+ CD45 ${ }^{\text {low }}$ microglia) and increased infiltration of CD4+ $\mathrm{T}$ cells during chronic phase of disease (Figure2K,S3K). 
Following uptake, exogenous squalene is transferred within the plasma and lipoprotein particles, adding to small amounts of endogenous serum squalene (Goodman, 1964; Gylling and Miettinen, 1994). Squalene can be converted to cholesterol by hepatic cells, especially when applied intravenously (Gabas-Rivera et al., 2014; Relas et al., 2001). However, when we measured peripheral cholesterol profile and squalene during pathology, we did not detect differences regarding total cholesterol (TC), Low-Density Lipoprotein cholesterol (LDL) or High-Density Lipoprotein cholesterol (HDL) but noticed a marked squalene increase in sera from $0.03 \mu \mathrm{g} / \mathrm{ml}$ in controls to $0.32 \mu \mathrm{g} / \mathrm{ml}$ in squalene treated EAE animals, indicating sufficient amount of compound availability (FigureS4D,S4E). Under healthy conditions the CNS is shielded from entry of peripheral lipids due to the BBB (Abbott et al., 2010; Saeed et al., 2014). In MS mouse models, we and others reported increased vascular permeability and BBB disruption, allowing entry of lipids (Bennett et al., 2010; Berghoff et al., 2017b; Munji et al., 2019; Paul et al., 2013). Therefore it is likely that squalene targets inflammatory cells not only in periphery but also in CNS tissue in EAE. To our surprise, squalene administration following cuprizone administration had no therapeutic effect, even in presence of BBB breach (FigureS4F,S4I)(Berghoff et al., 2017a). Likely explaining this conflicting result, we could not measure increased squalene concentration in serum of squalene treated cuprizone animals but detected increased TC and HDL-cholesterol levels (FigureS4G,S4H). Thus, it seems plausible that exogenous squalene is converted to cholesterol/cholesterol esters due to toxic liver damage induced by cuprizone, resulting in no therapeutic availability of squalene (Goldberg et al., 2013; Kipp et al., 2009; Suzuki, 1969).

To further investigate CNS related effects of exogenous squalene during inflammatory demyelination, squalene was applied therapeutically following disease onset avoiding interference with immunological priming phase of EAE (Paterka et al., 2017). In line with previous results, squalene treated animals exhibit reduced clinical disease severity and reduced number of infiltrating CD4+ T cells and CD11b+ CD45 low phagocytes, suggesting attenuated inflammatory lesion environment in CNS tissue (Figure3D,3E). In theory, loss of squalene synthase in phagocytes and exogenous squalene therapy interfere with equivalent downstream target genes in opposing manner. Therefore we isolated CD11b+ myeloid cells from SQScKO animals and squalene treated wildtype mice during toxic and inflammatory demyelination and integrated cell type specific and tissue related targeted expression profiles of genes associated with cholesterol synthesis, metabolism and inflammation (Figure3F,3G). Notably, we identified differential expression of inflammatory genes (Tnf, Nos2 and Tgfb1) as well as cholesterol efflux genes (Abca1 and Apoe) in addition to Fdft1 at the intersection (Figure $3 \mathrm{H})$. These findings support the possibility, that modulation of post-squalene cholesterol synthesis, either by interfering genetically or by exogenous squalene supplementation, impacts on inflammation and cholesterol efflux in phagocytes by utilizing an equivalent signaling pathway.

Targeting of LXR responsive genes through desmosterol rescues cholesterol export deficiency and proinflammatory gene signature in vitro

Myelin phagocytosis induces activation of the nuclear receptor LXR connecting transcriptional regulation of cholesterol efflux (e.g. Abca1) and inflammatory gene repression (e.g.Tnf, Nos2) (Bogie et al., 2012; Grajchen et al., 2018). In line with this, foam cell formation that we identified in squalene synthase deficient phagocytes (Figure 2E) has been attributed to inefficient LXR activation (Cantuti- 
Castelvetri et al., 2018; Schuster et al., 2002). Integrating post squalene cholesterol synthesis and LXR activation, sterol intermediates with LXR receptor ligand function have been identified in phagocytes (Shibata and Glass, 2009; Spann et al., 2012; Yang et al., 2006). In particular, lipid loading has been suggested to specifically reduce expression of the terminal cholesterol synthesis gene Dhcr24 leading to accumulation of the LXR ligand desmosterol thereby inducing cholesterol efflux and limiting inflammation (Spann et al., 2012). By analyzing cell type specific targeted expression profile of $\mathrm{CD} 11 \mathrm{~b}+$ phagocytes from demyelinated corpus callosum samples, we identified partial upregulation of cholesterol synthesis genes with prominent downregulation of Dhcr24 in vivo (Figure1G). To evaluate this mechanistically, we induced phagocytosis in bone marrow derived phagocytes by myelin incubation during high lipid conditions, when cholesterol synthesis is repressed. Strikingly, we identified dose dependent repression of Dhcr24 transcripts following phagocytosis correlating with increased expression of LXR target genes (Abca1, Apoe)(Figure4A), while other tested cholesterols synthesis genes indicated stable basal repression (FigureS5A). In line with our in vivo findings, we found insufficient induction of cholesterol export gene expression in SQScKO phagocytes (FigureS5C,S5D). Residual LXR target gene response in SQScKO phagocytes could be associated with induced expression of genes catalyzing conversion to oxysterol LXR ligands (Torocsik et al., 2009). Thus, we analyzed expression of Ch25h displaying highest transcript levels among genes associated with oxysterol synthesis in isolated phagocytes from the CNS (Figure S1B) and transcriptionally regulated during demyelination (Olah et al., 2012)(Figure1F,3G). Analysis revealed increased expression of Ch25h in SQScKO phagocytes in a myelin dose dependent manner, likely explaining residual Abca1 expression and protein abundance in vivo (Figure2C,2G), in addition to less severe pyroptotic cell death following chronic myelin ingestion in comparison to $A B C 1$ cKO phagocytes (Figure3l). Interestingly, this potential compensatory mechanism enabling partial cholesterol efflux might contribute to inflammation (Jang et al., 2016; Pokharel et al., 2019).

Within demyelinating CNS tissue, phagocytes encounter an inflammatory environment likely influencing metabolic processes. To account for that, SQScKO phagocytes were activated with a nonlethal concentration of LPS (FigureS5E,S5F) following low dose myelin phagocytosis induction under low lipid conditions, stimulating cholesterol synthesis. In line with previous results, we found inefficient upregulation of cholesterol efflux genes and increased expression of pro-inflammatory genes in the presence of Dhcr24 repression during late phase phagocytosis (Figure4B). To characterize earlyintermediate LXR target gene regulation and modulation of post squalene sterol synthesis in phagocytes, cells under defined in vitro conditions were activated and treated with low dose myelin (Figure4C). During initial phagocytosis with no impact on cell survival (FigureS5G), phagocytes induced the expression of cholesterol synthesis genes as previously suggested (Nordskog et al., 1999; Oiknine and Aviram, 1992; Posokhova et al., 2008), while Dhcr24 transcripts were repressed (FigureS5H). As expected, phagocytotic lipid loading induces expression of LXR target genes (Figure4D,4E) correlating with higher abundance of the LXR agonist desmosterol (Figure4J) in similarity to cholesterol loading of macrophages (Spann et al., 2012). Consistently, SQScKO cells showed failed accumulation of the LXR ligand desmosterol (Figure4J) correlating with inefficient induction of cholesterol efflux (Figure4D,4E) and increased inflammatory response (Figure4F,4G,4H). As expected, detected desmosterol accumulation was independent of cholesterol abundance, likely 
reflecting uptake of lipid rich myelin (Figure4I). In vivo, comparative targeted transcriptional profiling hints towards interference with equivalent downstream target genes by loss of squalene synthase and exogenous squalene therapy $(F i g u r e 3 H)$. In line, squalene administration rescued failed LXR target gene expression (Figure4D,4E) and relieved increased expression of inflammatory genes in SQScKO phagocytes (Figure4F,4G,4H) concomitant with increasing abundance of desmosterol (Figure4J). In addition, desmosterol accumulation and LXR target gene regulation could be enhanced in wildtype phagocytes (Figure4D-J). Interestingly, repression of inflammatory genes following squalene treatment could also be observed in cholesterol export deficient cells (ABC1cKO)(Figure $4 \mathrm{G}, 4 \mathrm{H})$, supporting previous observations of partial inflammatory gene suppression by LXR ligands independent of ABCA1(Thomas et al., 2018).

Desmosterol has been suggested to be the major LXR ligand in lipid loaded phagocytes. However, direct induction of LXR activation by squalene in macrophages has been reported (Hien et al., 2017). Therefore we compared the desmosterol mimetic DMHCA inducing prominent inflammatory repression (Figure4K) by LXR target gene regulation (Muse et al., 2018; Quinet et al., 2004) and squalene, with regards to cholesterol efflux gene induction without lipid loading. Analysis revealed an increase of Abca1 transcripts in non-activated and activated phagocytes up to $\sim 8.1$ fold following DMHCA treatment (FigureS5I). In contrast we did not observed induction of Abca1 expression by squalene in non-activated phagocytes while we noted a $\sim 1.5$ fold increase following activation (FigureS5I). Even considering possible slight LXR induction independent of desmosterol accumulation, prominent LXR target gene regulation seen in phagocytosis stimulated cells is unlikely attributed to direct LXR activation by squalene. SQScKO phagocytes displayed increased expression of Nos2 under high lipid conditions that even increased during lipid starvation (FigureS5J). This was reflected by higher ROS production (FigureS5K) that could be partially rescued by squalene supplementation following activation without lipid loading in wildtype and SQScKO phagocytes (FigureS5L). Therefore, it is possible that loss of squalene synthase and squalene supplementation interferes with other processes beside the regulated accumulation of desmosterol following myelin phagocytosis in vitro. Nonetheless, these data support the hypothesis that squalene supplementation can be applied to increase desmosterol accumulation in phagocytes, leading to enhance expression of LXR target genes thereby enabling cholesterol efflux and repression of inflammation (Figure4L,S9).

\section{Dhcr24 downregulation and accumulation of the LXR ligand desmosterol is shared between different MS mouse models and human disease}

Interference with post squalene cholesterol synthesis elucidates Dhcr24 downregulation and accumulation of the LXR ligand desmosterol as possible regulator of inflammation and cholesterol efflux, raising the question of the generality of this finding with regards to demyelinating pathologies. To test this, we compared expression in isolated CD11b+ phagocytes from cuprizone (Figure $5 A$ ), EAE (Figure5B), genetically induced (Figure5C) and lysolecithin induced (Figure5D) demyelinated lesions. By analyzing key genes of cholesterol synthesis (Figure5E), we found prominent repression of the regulatory candidate Dhcr24 in all analyzed samples (Luu et al., 2014; Sharpe and Brown, 2013). Consistently, accumulation of desmosterol was not only present in tissue (Figure5F), but also in isolated cells (Figure5G) from demyelinated lesions. Further, cholesterol abundance in isolated 
phagocytes was robustly elevated suggesting phagocytotic lipid loading during demyelination (FigureS6A). In contrast, we found reduced abundance of the sterol intermediates lanosterol and lathosterol in tissue samples of demyelinating mouse models (FigureS6B-S6D), indicating prominent myelin loss. Suggesting functional relevance, desmosterol accumulation was correlating with increased expression of the LXR dependent cholesterol efflux genes Abca1 and Apoe in isolated phagocytes from demyelinated tissue (FigureS6E). In addition, squalene synthase ablation leads to reduction of accumulated desmosterol as well as other cholesterol intermediates (FigureS6B-S6D) correlating with insufficiency of LXR dependent cholesterol efflux gene induction in isolated phagocytes in vivo (Figure $3 \mathrm{H}$ ). We then assessed desmosterol abundance in tissue of squalene treated EAE animals. As expected, squalene supplementation leads to higher abundance of desmosterol in tissue that is in line with increased expression of cholesterol efflux genes, repression of inflammatory genes and ameliorated clinical disease (FigureS6F,3H). Therefore, we suggest that increased squalene availability augments a conserved pathway in phagocytes of demyelinated lesions consequently limiting inflammation and thereby serving as a putative therapeutic target.

To examine the potential relevance of this finding in human MS disease, we analyzed single-cell gene expression profiles acquired from MS patients of two external studies (Jakel et al., 2019; Masuda et al., 2019). Four cell clusters were defined and visualized using UMAP in two-dimensional space (Figure5H,S7A). According to distribution between patients and the relative expression of selected gene sets we annotated cell cluster 4 , mostly occupied by cells from MS patients with presence of CD3 and S100A4 T cells, as principal adaptive immune cells cluster (FigureS7B,S7C). Cluster 3, evenly distributed among control and MS patients revealed high expression levels of PLP1 and other oligodendrocyte specific marker genes (data not shown) supporting occupancy of this cluster by recently identified immune oligodendroglia (FigureS7B,S7C)(Jakel et al., 2019). Interestingly, MS related cluster 2, attributed to phagocytes, displayed high transcript levels of $A B C A 1$ and $A P O E$ in contrast to non-MS patient cluster 1, annotated as homeostatic inflammatory cell cluster (Figure5H,S7B-S7D). Comparative analysis of cluster 2 reveal highest relative transcript levels of genes related to cholesterol export (marked in yellow) supported by identification of pathways involved in lipid transport following Gene Set Enrichment Analysis (Figure5H). Thus the annotated lipid export cluster 2 was characterized by repression of $D H C R 24$, increased expression of LXR regulated cholesterol efflux genes (ABCA1 and $A P O E)$ and LXR encoding genes (NR1H2 and NR1H3). In addition, lipid export cluster 2 was marked by reduced proportion of $D H C R 24$ positive cells and increased proportion of $A B C A 1$ and $N R 1 H 3(L X R \alpha)$ positive cells (Figure $5 H$ ).

Previous reports support induction of LXR signaling in phagocytes in active MS lesions (Mailleux et al., 2018). In addition, comparison of chronic active and inactive lesion rim as well as peri-lesion NAWM material revealed repression of Dhcr24 under the top 15 differential expressed genes correlating with phagocytosis and lipid efflux (Hendrickx et al., 2017). Exploiting this, we analyzed human MS lesion material for accumulation of the LXR ligand desmosterol. To do so, lesion center and lesion rim were dissected paralleled by histopathological characterization of myelin loss (LFB/PAS) and phagocytotic activity (KiM1P) as well as evaluation of tissue water content (Figure5I,S7E,S7F), indicating inflammation induced tissue swelling (Wang et al., 2015). Analysis revealed severely reduced abundance of cholesterol compared to non-MS tissue control samples, likely reflecting demyelination 
with no significant difference between dissected regions (Figure5I,S7G). Reflecting inflammatory activity of lesion material, we found significantly increased tissue water in active lesion samples (FigureS7F). Strikingly, when compared to non-MS control, we detected significantly higher amounts of desmosterol in tissue samples of the active lesion rim (Figure5l). In contrast desmosterol abundance was reduced in the center of inactive lesions (Figure5I). Cumulatively, these data suggest that accumulation of the LXR ligand desmosterol due to transcriptional repression of DHCR24 in phagocytes of active MS lesions correlates with LXR activation and downstream target gene regulation, controlling cholesterol efflux and inflammation.

Squalene administration induces myelin formation and can be applied in combination therapy Besides the importance of interfering with detrimental inflammatory responses in phagocytes during demyelinating episodes, remyelination therapy targeting oligodendroglia to enable regeneration, has become a major objective in MS (Franklin and Ffrench-Constant, 2008; Rodgers et al., 2013). Cholesterol availability is a prerequisite for myelination and directly enhances remyelination (Berghoff et al., 2017b; Saher et al., 2005; Saher et al., 2012; Stumpf et al., 2019). Squalene as a cholesterol synthesis intermediate can be converted to cholesterol. Therefore, increased availability of squalene could partially relieve oligodendrocytes of the burden to establishing the complex time- and energyintensive anabolic cholesterol pathway. Thus, we tested squalene supplementation during in vitro myelination in spinal cord co-cultures. As expected, rate of myelination measured by MBP (myelin basic protein) positive axons was significantly increased by squalene suggesting a direct effect on oligodendrocytes (Figure6A). To investigate remyelination potential of squalene in vivo, lysolecithin induced focally demyelinated animals were supplemented with squalene. Examination of serum samples demonstrated increased squalene availability with no alteration with regarding peripheral cholesterol (FigureS8A,S8B). In line, squalene administration following lysolecithin induced demyelination increased number of CAll+ differentiated oligodendrocytes as well as MBP positive lesion area, indicating enhanced remyelination (Figure6B).

We next tested the therapeutic potential of squalene during inflammatory demyelination in combination therapy. Therefore, squalene supplementation was combined with daily injections of the antiinflammatory first-line therapeutic compound IFN $\beta-1 \mathrm{~b}$. Notably, combination therapy reduced clinical disease severity in comparison to therapy only treated groups, proving feasibility and safety of this treatment paradigm (FigureS8C). Interestingly, we previously reported no impact of cholesterol on inflammatory disease. However, cholesterol loading in phagocytes induces Dhcr24 repression as well as increased expression of LXR target genes (Spann et al., 2012), that we detected in EAE tissue of cholesterol treated mice (FigureS8D,S8E). While acting in general anti-inflammatory through not fully elucidated mechanisms, IFNß-1b induces phagocytosis in microglia (Kocur et al., 2015). Thus, we hypothesized the possibility of a masked effect of cholesterol loading on phagocytes potentially inducing beneficial phenotypic switching in EAE. Therefore, combination therapy of exogenous cholesterol and daily IFN $\beta-1 \mathrm{~b}$ treatment was tested. Supporting this possibility, we found reduced number of inflammatory microglia/macrophages within CNS tissue as well as reduced expression of pro-inflammatory genes and amelioration of clinical disease in animals treated with cholesterol combination therapy (FigureS8F-S8H). 
Nonetheless, squalene therapy has revealed both, robust anti-inflammatory effects and a direct supporting effect on oligodendrocyte myelination. To further explore the potential of lipids in combination therapy, we combine squalene with suboptimal dosing of the LXR ligand DMHCA and daily IFN $\beta-1 b$ injections. Clinical examination revealed a robust amelioration of disease severity in triple therapy treated mice compared to double, single and no treatment controls (Figure6C). Moreover, analysis of inflammatory cells isolated from CNS tissue reflected reduced disease severity (Figure6D). Because of suboptimal dosing of DMHCA, we expected synergistic LXR target gene regulation due to enhanced endogenous desmosterol accumulation previously detected. Therefore, $\mathrm{CD} 11 \mathrm{~b}+$ phagocytes were isolated from either DMHCA or DMHCA + squalene combination therapy treated EAE mice. Accordingly, DMHCA induced regulation of LXR target genes leading to increased Abca1 transcript levels, repression of proinflammatory genes and induction of anti-inflammatory mediator gene expression, that was further augmented in squalene + DMHCA combination therapy treated EAE mice (Figure6E,S8I). Taken together, in inflammatory models of demyelination squalene therapy reduces disease severity by inducing LXR activation thereby suppressing inflammation in addition to directly supporting myelination in oligodendroglia (FigureS9). Furthermore, squalene therapy can be combined with the LXR ligand DMHCA and the first-line medication IFN $\beta-1 \mathrm{~b}$ suggesting implications for therapeutic intervention in MS (Figure6F).

\section{Discussion}

Oligodendrocyte (OL) cholesterol synthesis is rate-limiting for myelination (Saher et al., 2005). In this study, our data substantiate the requirement of cholesterol availability in oligodendrocytes during remyelination in particular during chronic phase of disease, with therapeutic relevance for neurodegenerative disorders like MS (Berghoff et al., 2017b; Voskuhl et al., 2019). However, acute remyelination did not rely on cholesterol synthesis in oligodendroglia nor in astrocytes or endothelial cells. Unexpectedly, functional cholesterol synthesis in phagocytes dictated remyelination efficiency. Together with previous studies, recycling of cholesterol by phagocytes following myelin clearance has been suggested (Lavrnja et al., 2017; Olah et al., 2012), likely supporting OL differentiation and myelination (Berghoff et al., 2017b). Leaving the incorporation route of cholesterol unsolved, we did not observe upregulation of classical LDL or VLDL receptors in oligodendrocytes that have been linked to developmental myelination (Zhao et al., 2007). Possibly, there is involvement of one, out of several other cholesterol receptor families that have not been analyzed (Lane-Donovan et al., 2014) or direct incorporation of cholesterol into myelin membranes as we have previously shown (Saher et al., 2012)

Nonetheless, our study identifies chronic activation and cholesterol efflux deficiency in phagocytes with deficient post squalene cholesterol synthesis. Interestingly, cholesterol export deficiency (ABC1CKO) in phagocytes results in extensive lipid loading and pyroptotic cell death (CantutiCastelvetri et al., 2018) that we partially observed in cholesterol synthesis mutant cells (SQScKO). In contrast, remyelination and extensive OL differentiation in ABC1cKO animals was absent in SQScKO mice. Previous studies have highlighted the role of active phagocyte cell death to enable remyelination (Lloyd et al., 2019). Therefore, it is possible that pyroptotic cell death of ABC1cKO phagocytes induces 
partial remyelination while chronic activation with moderate cell death $\sim 50 \%$ of SQScKO cells impairs regeneration.

However, lipid loading in phagocytes and export deficient ABC1cKO cells induce an anti-inflammatory phenotype (Bogie et al., 2012) that we did not observe in SQScKO phagocytes. Thus, we hypothesized a direct relation of post squalene cholesterol synthesis, inflammation and cholesterol efflux. Several studies suggest a link between cholesterol synthesis and phagocyte functioning (Araldi et al., 2017; Shibata and Glass, 2009; Spann et al., 2012; Yang et al., 2006). In macrophages, lanosterol accumulation has been linked to innate immune response (Araldi et al., 2017). In contrast, accumulation of the LXR ligand desmosterol following cholesterol loading in atherosclerosis associates with acquirement of anti-inflammatory profiles in macrophages (Spann et al., 2012). Here, based on genetic and compound based interference with post squalene cholesterol synthesis in vitro and in vivo, we identified a conserved pathway leading to desmosterol accumulation and induction of LXR target genes in microglia/macrophages as important regulator to suppress inflammation, induce cholesterol efflux and enable remyelination following myelin phagocytosis. In addition our data provides evidence of this mechanism in human MS disease. Intriguingly, active phagocyte LXR signaling in MS lesions has been reported (Mailleux et al., 2018). Therefore, these observations will be of interest with respect to genetic variants associated with MS disease, in particular identified variants of $N R 1 H 3(L X R \beta)$ and potentially FDFT1 in relation to phagocyte functioning (Smets et al., 2018; Wang et al., 2016).

In MS, there is urgent need for therapeutic strategies targeting inflammation and remyelination either by single compounds or combination therapy (Franklin and Ffrench-Constant, 2008; Rodgers et al., 2013). In our study, we expanded conceivable therapeutic implications and tested combinational therapy with the lipid precursor squalene, feeding into accumulation of the LXR ligand desmosterol in phagocytes (anti-inflammatory mode of action) and supporting myelination in oligodendrocytes (remyelinating mode of action). To validate therapeutic options, we also tested the possibility of synthetic LXR agonist application as previously reported (Secor McVoy et al., 2015; Solt et al., 2012) . Since first generation LXR agonists e.g. GW3965 come along with peripheral side effects like enhanced lipogenesis, hypertriglyceridemia, and liver steatosis(Hong and Tontonoz, 2014; JamrozWisniewska et al., 2007), we applied the desmosterol mimetic DMHCA selectively targeting LXR in macrophages (Muse et al., 2018). Together these data provide evidence of a therapeutic disease ameliorating effect targeting LXR in phagocytes either directly by synthetic LXR agonists or indirectly by squalene supplementation as well as in combination with first-line anti-inflammatory MS medication. Although we prove therapeutic implications and safety of squalene in combination therapy in several MS mouse models, studies in MS patients have to be conducted. Interestingly, Mediterranean diets (Katz Sand, 2018; Sedaghat et al., 2016) and olive oil consumption (Rezapour-Firouzi et al., 2013) have been linked to reduced disease severity in MS. Of note, olive oil contains high amounts of squalene of up to $\sim 0.8 \%$ (Beltrán et al., 2016) and results in average consumption levels in Mediterranean counties of $200-400 \mathrm{mg} /$ day in contrast to $<30 \mathrm{mg} /$ day in the US (Smith, 2000). In addition, dietary squalene administration has proven to be safe in humans (Chan et al., 1996; Gylling and Miettinen, 1994). Therefore, these findings emphasis the need as well as chances of therapeutic studies by combination therapy with squalene. 


\section{Limitations of the Study}

The expression profiles in this study have been obtained via MACS $₫$ based acute cell isolation. Different sorting methods vary with regards to throughputs, yields, viabilities, and processing times thereby influencing results obtained and partially engender inter-study variability (Sutermaster and Darling, 2019). In addition, antibody based labeling results in a bias by reducing the complexity of cell populations. Therefore, the present data have to be interpreted as bulk mRNA measurements that lack the information of cell subpopulations (Hwang et al., 2018).

In MS, there is evidence of extensive cortical grey matter pathology that differs with regards to the inflammatory environment from white matter lesions (Stadelmann et al., 2008). In this study, we analyzed pathology in mouse models of MS that mainly reflect white matter demyelination. Therefore, we cannot conclude on heterogeneity of cell types or pathology from specific brain regions. Thus, single-cell RNA sequencing in combination with region-specific transcriptomics is a key future objective.

\section{Acknowledgments}

We are grateful to Klaus-Armin Nave for constant support. We cordially thank Annette Fahrenholz, Tanja Freerck, Boguslawa Sadowski and Laura Fernandez Garcia-Agudo for technical support. We thank Prof Charles Stiles, Dr John Alberta, Prof Said Ghandour and Prof. Dr. Radzun for generous gifts of antibodies. This work was funded by the Deutsche Forschungsgemeinschaft (SA 2014/2-1 to G.S.). J.E. and W.M. were funded by an ERC Advanced grant awarded to Klaus-Armin Nave.

\section{Author Contributions}

S.A.B. and G.S. planned and designing the study.

S.A.B. and L.Sp. were involved in all experiments. T.S. performed reanalysis of human sc-RNAseq datasets. L.Sc. performed cell culture experiments. L.H. and F.O. did flow cytometry with subsequent expression analyses. T.D. was involved in MACS based cell isolation. D.E. performed FPLC serum analysis. J.N. did western blot analysis T.R. performed electron microscopy analysis. T.I and P.S. performed lipid mass spectrometry. C.SN. and F.P. characterized and isolated human MS tissue lesion. L.C. and M.S. were in involved in lysolecithin experiments. W.M and M.M. characterized mouse pathology. J.M.E performed myelinating co-culture experiments. C.D. and A.O.S. did light sheet microscopy.

S.A.B., KA.N. and G.S. wrote and edited the manuscript.

\section{Declaration of Interests}

The authors declare no competing financial interests. 


\section{Main figures}

Figure 1.Cholesterol metabolism during de- and remyelination

(A) Scheme depicting the time course of de- and remyelination (upper panel, based on own results and on other studies) to cuprizone treatment paradigm (Matsushima and Morell, 2001).

(B) Representative pictures of the corpus callosum during cuprizone induced acute and chronic deand remyelination (Myelin visualized with Gallyas silver staining).

(C) Flowchart depicting experimental design of the study.

(D) Scheme of applied cell isolation procedure (MACS).

(E) Comparison of Hmgcr gene expression in different cell types between datasets .

(F) Targeted sterol gene expression profile of corpus callosum samples $(n=4)$ during acute and chronic remyelination. Heat map shows fold expression value of single samples normalized to untreated controls ( $n=4$; set to 1$)$.

(G) Targeted sterol gene expression profile of isolated cells from individual animals $(n=4)$ during acute and chronic remyelination. Fold expression heat map normalized to isolated cells from control $(n=4$; set to 1$)$.

(H) Remyelination efficiency of cell specific cholesterol synthesis mutants during acute and chronic remyelination evaluated by histological staining's for myelin (Gallyas), mature oligodendrocytes (CAII), microgliosis (MAC3) and astrogliosis (GFAP). Controls set to $100 \%$ (not depicted). Single data points represent individual animals analyzed $(n=3-7)$. Labeling indicates significantly affected clusters of cholesterol synthesis mutants with impaired remyelination efficiency and/or alter gliosis

Figure 2. Microglial cholesterol synthesis ablation causes persistent activation and cholesterol export deficiency

(A) Scheme depicting analyzed control and conditional cholesterol synthesis and export mutants.

(B) Comparison of microglial specific cholesterol synthesis (SQS) and cholesterol export (ABC1) mutants during acute remyelination. Representative pictures (left) of myelin (Gallyas), mature oligodendrocytes (CAII), oligodendrocyte linage cells (OLIG2) and microgliosis (MAC3) with corresponding quantification (right) (Scale $50 \mu \mathrm{m}$ ). Bars represent means of individual animals ( $n=3-4)$ normalized to cuprizone control ( $n=3-4$, set to 100). ${ }^{* * *} p<0.001,{ }^{* *} p<0.01,{ }^{*} p<0.05$.

(C) Expression of genes related to oligodendrocyte differentiation (Car2, Olig2) cholesterol export (Abca1, Abcg1) and inflammation (right) in the corpus callosum during acute remyelination. Data normalized to cuprizone control (set to 1 ) ${ }^{* * *} p<0.001$, ${ }^{* *} p<0.01$, ${ }^{*} p<0.05$.

(D) In vitro phagocytosis of myelin in BMDM cultures. (upper panel) Representative pictures (Scale 5 $\mu \mathrm{m}$ ) of LAMP1/Fluormyelin co-labelling (left) with corresponding quantification (right) of control and SQScKO ( $n=4-7,600-1050$ cells) after 4 h myelin treatment.

(E) Appearance of foamy microglia in microglial SQScKO animals during acute remyelination shown by MAC3 (upper panel, scale $50 \mu \mathrm{m}$ ) and Methylen blue (middle, scale $10 \mu \mathrm{m}$ ) staining

(F) Occurrence of cholesterol crystals, visualized by electron microscopy in microglial SQScKO animals (Scale $1 \mu \mathrm{m})$

(G) Western Blot analysis of ABCA1 expression in WT, cuprizone control and cuprizone SQScKO animals $(n=3) .{ }^{* * *} p<0.001,{ }^{* *} p<0.01$.

$(\mathrm{H})$ Expression of genes related to inflammasome activation (NIrp3, Casp1 and II1b) and cholesterol export (Abca1) in isolated microglia ( $n=3-5)$ during acute demyelination (5 weeks cuprizone). ${ }^{* *} p<$ $0.01,{ }^{*} p<0.05$.

(I) Live phagocytosis imaging of control, SQScKO, ABC1cKO and APOE-/- BMDMs treated with myelin for $16 \mathrm{~h}$. Individual data points represent analyzed cells over $16 \mathrm{~h}$ treatment period $(\mathrm{n}=12$ 19). ${ }^{* * *} p<0.001$.

(J) Representative pictures (Scale $100 \mu \mathrm{m}$ ) of persistent microgliosis stained with MAC3 (upper left) or IBA1/LAMP1 co-labeling (lower left) in lysolecithin lesions of the spinal cord from SQScKO animals $(n=7)$ and controls $(n=6)$ with corresponding quantification of lesions (SQScKO $n=12$, control $n=14$ ) on the right.

(K) Clinical score \pm SEM of EAE control $(n=34)$ and SQScKO $(n=27)$ mice with quantification of myeloid inflammatory cells within CNS tissue. 
Figure 3. Squalene administration suppresses EAE and induces upregulation of cholesterol efflux genes

(A) Scheme depicting genetic and peripheral interference with cholesterol pathway in EAE.

(B) Clinical score of control $(n=34)$ and SQScKO $(n=27)$ mice (left), and control $(n=6)$, SQScKO $(n=11)$ and SQScKO squalene $(n=5)$ treated mice (right) with MOG-EAE. Mice received squalene diet commencing at 14 days before immunization.

(C) Absolute number of CD4+ and CD8+ $T$ cells, CD11b/CD45high macrophages and CD11b/CD45low microglia per gram spinal cord determined by flow cytometry at chronic EAE (28 days) of animals in (A)( left panel, $n=8)$. ${ }^{* * *} p<0.001,{ }^{* *} p<0.01$.

(D) Clinical score \pm SEM of prophylactically (left) and therapeutically (right) squalene treated EAE animals. Prophylactic: control $(n=7)$ and squalene $(n=9)$. Therapeutic: control $(n=10)$ and Squalene $(n=11)$.

(E) Analysis of inflammatory cells in spinal cord tissue by flow cytometry from animals in (D) (right panel; $n=6) .{ }^{* *} p<0.01,{ }^{*} p<0.05$.

(F) Targeted expression profile in tissue of microglial SQScKO animals during cuprizone (corpus callosum, acute, $n=5$ ), EAE (spinal cord, chronic, $n=5$ ) and squalene treated EAE (spinal cord, chronic, $n=6$ ). Heat map shows fold expression values of individual animals normalized to corresponding controls (set to 1 ).

(G) Targeted expression profile of CD11b+ isolated myeloid cells of experimental groups in (E).

$(H)$ Venn diagram of significantly regulated genes in $(E)$ and $(F)$ revealing cholesterol efflux genes (Abca1, Apoe) and inflammatory genes (Tnf, Nos2, Tgfb1) at the intersection.

Figure 4.Squalene treatment rescues failed LXR target gene upregulation and ameliorates inflammation in SQScKO BMDMs after myelin phagocytosis

(A) Expression of Dhcr24, Hmgcr, Abca1 and Apoe in BMDMs after 4h treatment with increasing dose of myelin. Bars represent mean of individual cultures normalized to untreated control (set to 1).

(B) Expression of genes related to cholesterol synthesis, export and inflammation in control and SQScKO BMDMs treated with myelin $(10 \mathrm{mg} / \mathrm{ml})$ on the left and LPS $(10 \mathrm{ng} / \mathrm{ml})+$ myelin on the right. Heap map shows fold expression values of individual cultures normalized to corresponding control (set to 1).

(C) Scheme depicting squalene $(100 \mu \mathrm{M})$, LPS $(10 \mathrm{ng} / \mathrm{ml})$ and myelin treatment paradigm in vitro.

(D) Expression of Abca1 after LPS and myelin stimulation for $4 \mathrm{~h}$ in control, SQScKO and ABC1cKO BMDMs treated either with or without squalene $(100 \mu \mathrm{M})$.

(E) Expression of Abcg1 after LPS and myelin stimulation for $4 \mathrm{~h}$ in control, SQScKO and ABC1cKO BMDMs treated either with or without squalene $(100 \mu \mathrm{M})$.

(F) Expression of Tnf after LPS and myelin stimulation for $4 \mathrm{~h}$ in control, SQScKO and ABC1cKO BMDMs treated either with or without squalene $(100 \mu \mathrm{M})$.

(G) Expression of $\| 1 \mathrm{~b}$ after LPS and myelin stimulation for $4 \mathrm{~h}$ in control, SQScKO and ABC1cKO BMDMs treated either with or without squalene $(100 \mu \mathrm{M})$.

(H) Expression of Nos2 after LPS and myelin stimulation for $4 \mathrm{~h}$ in control, SQScKO and ABC1cKO BMDMs treated either with or without squalene $(100 \mu \mathrm{M})$.

(I) Measurement of cholesterol normalized to cell number in LPS and myelin stimulated control and SQScKO BMDMs measured by lipid mass spectrometry.

(J) Measurement of desmosterol normalized to cell number in LPS and myelin stimulated control and SQScKO BMDMs measured by lipid mass spectrometry.

(K) Treatment of stimulated BMDMs with the desmosterol mimetic DMHCA $(10 \mu \mathrm{M})$ results in increased expression of Abca1 and reduced expression of the inflammatory genes Tnf and Nos2.

(L) Scheme depicting presumptive consequences induced by squalene synthase ablation (SQScKO), cholesterol efflux deficiency (ABCA1/G1cKO) and supplementation with squalene or DMHCA on LXR target gene expression.

Bars represent mean of individual cultures normalized to untreated control (set to 1 ). ${ }^{* * *} p<0.001$, ${ }^{* *} p<0.01,{ }^{*} p<0.05$. 
Figure 5. Dhcr24 downregulation and accumulation of the LXR ligand desmosterol is shared between different demyelinating mouse models

(A) Expression of cholesterol synthesis genes in isolated CD11b+ cells during acute (left) and chronic (right) cuprizone induced remyelination. Data points resemble individual samples $(n=4)$ normalized to untreated control (set to 1$) .{ }^{* *} p<0.01$.

(B) Expression of cholesterol synthesis genes in isolated CD11b+ cells from spinal cord during peak (left) and chronic (right) EAE. Data points resemble individual samples ( $n=4-6)$ normalized to untreated control (set to 1 ). ${ }^{* *} p<0.01,{ }^{*} p<0.05$.

(C) Expression of cholesterol synthesis genes in isolated CD11b+ cells from corpus callosum of MBPcKO (left, $n=4$ ). Data points represent individual samples normalized to untreated control $(n=3-4$, set to 1$) .{ }^{* * *} p<0.001,{ }^{*} p<0.05$

(D) Expression of cholesterol synthesis genes in isolated CD11b+ cells from spinal cord of lysolecithin induced (right, $n=3$ ) animals. Data points represent individual samples normalized to untreated control ( $n=3-4$, set to 1$) .{ }^{* *} p<0.01$

(E) Cholesterol synthesis pathway depicting cholesterol intermediates of the Bloch and KandutschRussel pathway with critical cholesterol synthesis genes. Pathway separation by Dhcr24 mediated $\mathrm{C} 24$ double bound side chain reduction is highlighted in red.

(F) Relative abundance of desmosterol normalized to cholesterol in tissue during acute cuprizone, chronic EAE and lysolecithin (day 7) determined by mass spectrometry. Bars represent mean of individual animals (data points) normalized to untreated control (set to 1 ). ${ }^{* * *} p<0.001,{ }^{* *} p<0.01$, ${ }^{*} p<0.05$.

(G) Relative abundance of desmosterol normalized to cell number in isolated CD11b+ microglia during acute cuprizone measured by mass spectrometry. Bars represent mean of individual samples (data points) normalized to untreated control (set to 1). ${ }^{*} p<0.05$.

(H) Reanalysis of human MS scRNAseq datasets. (upper left) Uniform Manifold Approximation and Projection (UMAP) plot of inflammatory cell clusters, sorted by cell population. (upper right) Mean expression of DHCR24, ABCA1, APOE, NR1H2 and NR1H3 in control and MS inflammatory cells between cluster 1 to 3 showing lowest expression (blue) and highest expression (red) and frequency distribution of DCHR24, ABCA1 and NR1H3 positive nuclei/cells. (lower left) Top 20 significantly upregulated genes of cluster 2 sorted by fold expression value. Genes related to cholesterol export are marked in yellow. (lower right) Gene Set Enrichment Analysis (GSEA) for differentially regulated genes between cell populations and significant ontology terms visualized in network showing appearance of pathways involved in cholesterol transport (yellow).

(I) Representative pictures of human MS lesion (upper panel, Scale 500 $\mu \mathrm{m}$ ) stained for myelin (LFB/PAS) and myeloid cell activation/infiltration (KiM1P). Dotted lines indicate lesion center and rim. (lower panel) Relative abundance of cholesterol (left) and desmosterol (right) normalized to input tissue weight and standard (controls set to 1). Individual data points represent lesions analyzed from non-MS controls $(n=3)$ and MS patients $(n=4)$. Ctrl: control white matter; R: Lesion rim; AC: Active lesion center; IC: Inactive lesion center. ${ }^{* * *} p<0.001,{ }^{*} p<0.05$.

Figure 6. Squalene supports remyelination and can be applied in combination therapy

(A) Myelination at 21 and 30 days in vitro (DIV) in myelinating cocultures in the presence or absence of squalene ( $n=7-9$ cultures). Representative images (left, Scale $50 \mu \mathrm{m}$ ) showing myelin segment stained with MPB (green) and axons visualized with SMI31 (blue) with corresponding quantification (right). ${ }^{* *} p<0.01,{ }^{*} p<0.05$.

(B) Representative pictures (Scale $100 \mu \mathrm{m}$ ) of mature oligodendrocytes labeled with CAll (upper left) and myelin stained with MBP (lower left) in lysolecithin lesion of the spinal cord in control and squalene treated animals with corresponding quantification on the right. ${ }^{* * *} p<0.001,{ }^{*} p<0.05$.

(C) Clinical score \pm SEM of EAE induced animals receiving control chow $(n=12)$, chow supplemented with DMHCA (12) or DMHCA + Squalene $(n=11)$ therapeutically or in combination with daily IFN $\beta$ $1 b$ injections $(n=10)$.

(D) Absolute number of CD4+ and CD8+ T cells, CD11b/CD45high macrophages and CD11b/CD45low microglia per gram spinal cord determined by flow cytometry at chronic EAE (28 days) of animals in (D). ${ }^{* *} p<0.01,{ }^{*} p<0.05$. 
(E) Expression of the LXR target gene Abca1 and inflammatory genes (Tnf, Nos2,Arg1 and Tgfb1) in isolated $\mathrm{CD} 11 \mathrm{~b}+$ cells from animals in (E). Data points resemble individual samples normalized to untreated control (set to 1 ). * $p<0.05$.

(F) Scheme depicting suggested compound targets of IFN $\beta-1 \mathrm{~b}, \mathrm{DMHCA}$ and Squalene with respect to effects on inflammation, LXR target gene regulation and remyelination.

\section{Supplemental Figures}

S. Figure 1. Generation and validation of cell specific cholesterol synthesis mutants, Related to figure 1

(A) Quantitative RT-PCR analysis determining the expression of oligodendrocyte and myelin related genes (Car2, Plp1, Olig2), marker genes for microglia (Aif1) and astrocytes (Gfap) in corpus callosum samples during acute and chronic remyelination. Bars represent the means with individual data points normalized to demyelination time point ( $n=4$, set to 1 , dotted line).

(B) Targeted sterol gene expression profile of isolated cells from the corpus callosum of wild type animals. Relative expression heat map of single samples representing low transcript levels (blue) and high transcript levels (red) for each gene between individual samples $(n=4)$.

(C) Fdft1 (SQS) expression in isolated cells of applied cholesterol synthesis mutants. (Upper panel) Applied Cre driver lines were crossbred to SQSflox/fllox or Tdto reporter mice. (Middle panel) Tamoxifen protocol with time points of analysis. (Lower panel) Fdft1 expression in targeted cells. Fold expression values of single samples $(n=3-4)$ normalized to control cells $(n=3-4$, set to 1$)$. ${ }^{* *} p$ $<0.001,{ }^{*} \mathrm{p}<0.05$.

(D) Bodyweight and peripheral total cholesterol of analyzed cell specific cholesterol synthesis mutants in (S1C) at the age of 22 weeks. Bars represent mean of individual animals ( $n=4-15$, data points) ${ }^{*} p<0.05$

(E) Tamoxifen protocol to induced recombination during cuprizone paradigm. Tamoxifen was given either before cuprizone application to target SQS in astrocytes, microglia, OPCs and endothelial cells or before remyelination to target oligodendrocytes.

(F) TDTO reporter expression during remyelination to evaluate cell targeting in cuprizone fed mice by co-labeling with CAll for oligodendrocytes, OLIG2 for oligodendrocyte linage cells, GFAP for astrocytes and IBA1 for microglia.

S. Figure 2. Generation and validation of cell specific cholesterol synthesis and MBP conditional mutants, Related to figure 1

(A) Expression of cholesterol synthesis genes in isolated astrocyte from EAE lesions (upper panel). Bars represent the means with individual data points $(n=4)$ normalized to isolated cells from control (set to 1). ${ }^{* *} p<0.01,{ }^{*} p<0.05$.

(B) Generation of MBP conditional mutants (MBPcKO)(upper panel) and expression of cholesterol synthesis genes in isolated astrocyte (lower panel) 12 month post tamoxifen administration (lower panel). Bars represent the means with individual data points $(n=4)$ normalized to isolated cells from control (set to 1$)$. ${ }^{* *} p<0.01,{ }^{*} p<0.05$.

(C) Evaluation of astrocyte Fdft1 expression, bodyweight and peripheral total cholesterol in astrocyte specific cholesterol synthesis mutants (see S.fig.1). Bars represent the means with individual data points $(n=3-8)$ normalized to controls. ${ }^{* * *} p<0.001$

(D) Histological quantification of myelination (Gallyas), mature oligodendrocytes (Call), oligodendrocyte linage cells (Olig2), microgliosis (MAC3) and astrogliosis (GFAP) in astrocyte cholesterol synthesis mutants during acute remyelination $(n=4-5)$. Bars represent the means with individual data points normalized to controls (set to 100).

(E) Elevated beam testing of cholesterol synthesis mutants during chronic remyelination $(n=5-8)$. Bars represent the means of 3 tested from individual animals.

S. Figure 3. Microglial cholesterol synthesis ablation does affect demyelination in the cuprizone model, Related to figure 2

(A) Generation and cuprizone treatment paradigm of Abca1/ Abcg1 phagocyte specific cholesterol export mutants. 
(B) Representative electron microscopic pictures of phagocyte SQScKO and control animals during acute remyelination (Scale $1 \mu \mathrm{m}$ ).

(C) Intracellular cholesterol labeling in SQScKO BMDMs under low lipid media (LDS) conditions by live staining with $\mathrm{BC}$ theta (Scale $10 \mu \mathrm{m})$.

(D) Fdft1 expression in SQScKO BMDMs under high lipid condition. ( $n=4)$. Data normalized to control cells (set to 1$) .{ }^{* *} p<0.01$.

(E) In vitro fluoromyelin phagocytosis after $4 \mathrm{~h}$ in LPS $(10 \mathrm{ng} / \mathrm{ml})$ stimulated BMDM cultures $(\mathrm{n}=4-7$, 600-1050 cells).

(F) Representative images of phagocyte SQScKO and control animals stained for oligodendrocytes (CAII), oligodendrocyte linage cells (OLIG2) and microgliosis (MAC3) after 5 weeks cuprizone administration (Scale $50 \mu \mathrm{m}$ ) with corresponding quantification on the right.

(G) Representative electron microscopic pictures of phagocyte SQScKO and control animals after acute demyelination (Scale $1 \mu \mathrm{m}$ ).

(H) Abca1 and Abcg1 expression in corpus callosum samples isolated from phagocyte cholesterol export mutants (ABC1cKO) during acute remyelination $(n=4) .{ }^{* * *} p<0.001,{ }^{* *} p<0.01$.

(I) Quantification of lesion area determined by DAPI positive increased cellularity in SQScKO animals following lysolecithin lesion induction (14 days dpi) (Scale $100 \mu \mathrm{m}$ ).

(J) Tamoxifen protocol to induce recombination specifically in microglia during EAE.

(K) Quantification of inflammatory cells within CNS tissue of EAE induced SQScKO animals during chronic phase of disease ( 28 days). Bars represent the means with individual data points $(n=8)$ ${ }^{* * *} p<0.001$

\section{S. Figure 4. Squalene ameliorates EAE but shows no effect on remyelination in the cuprizone} model, Related to figure 3

(A) Clinical score \pm SEM of control $(n=6)$ and prophylactically squalene $(n=6)$ treated EAE animals analyzed at peak of disease.

(B) Absolute number of CD4+ and CD8+ T cells, CD11b/CD45 ${ }^{\text {high }}$ macrophages and CD11b/CD45 ${ }^{\text {low }}$ microglia per gram spinal cord determined by flow cytometry at chronic EAE (28 days) of control and squalene treated animals $(n=6)$ treated with squalene (A, left panel). ${ }^{*} p<0.05$.

(C) Analysis of inflammatory cells in collected blood $(n=5-6)$ by flow cytometry from animals in (B) during peak of disease.

(D) Peripheral serum cholesterol profile of control and squalene treated EAE animals during peak of disease $(n=10-11)$. Measurement shows total cholesterol (TC), low density lipoprotein cholesterol $(\mathrm{LDL})$ and high density lipoprotein cholesterol (HDL). Dotted line indicates values of non-disease controls.

(E) Squalene serum concentration of control and squalene treated $E A E$ animals during peak of disease measured by FPLC ( $n=2-3)$.

(F) Scheme depicting induced remyelination paradigm. Cuprizone was removed from the diet after 4 weeks and mice received either control or squalene supplement chow for one week.

(G) Peripheral serum cholesterol profile determining total cholesterol (TC), low density lipoprotein cholesterol (LDL) and high density lipoprotein cholesterol (HDL) in cuprizone animals supplement with or without squalene $(n=4-5)$. Dotted line indicates values of non-disease controls. ${ }^{* * *} p<0.001$

(H) Squalene serum concentration measured by FPLC of control and squalene treated cuprizone animals $(n=3)$. Dotted line indicates values of non-disease controls.

(I) Histological quantification of myelination (Gallyas), mature oligodendrocytes (Call), oligodendrocyte linage cells (Olig2), microgliosis (MAC3) and astrogliosis (GFAP) in squalene.

S. Figure 5. Cholesterol synthesis/export gene regulation and inflammatory response in wildtype and SQScKO BMDMs challenged with myelin, Related to figure 4

(A) Expression of cholesterol synthesis genes in wildtype BMDMs after $4 \mathrm{~h}$ myelin phagocytosis with increasing dose $(n=3)$ under high lipid conditions (FCS). Data normalized to high lipid control condition (set to 1 ).

(B) Expression of $\mathrm{CH} 25 \mathrm{~h}$ in control and SQScKO BMDMs after $4 \mathrm{~h}$ myelin phagocytosis with increasing dose $(n=3)$ normalized to untreated control (set to 1$)$. 
(C) Expression of $\mathrm{Hmgcr}$ and Dhcr24 in control and SQScKO BMDMs after 4 h myelin phagocytosis with increasing dose $(n=3)$ normalized to untreated control (set to 1).

(D) Expression of Abca1 and Abcg1 in control and SQScKO BMDMs after 4 h myelin phagocytosis with increasing dose $(n=3)$ normalized to untreated control (set to 1).

(E) Expression of the inflammatory mediator genes Tnf and $1 / 1 \mathrm{~b}$ after $4 \mathrm{~h}$ LPS treatment with increasing dose $(n=3)$ normalized to untreated control (set to 1).

(F) Cell viability assay (WST-1) indicating no cell death induced by selected LPS concentration of 10 $\mathrm{ng} / \mathrm{ml}(\mathrm{n}=3)$ within $18 \mathrm{~h}$ compared to untreated control ( $\mathrm{n}=6$, set to 1$). \mathrm{H}_{2} \mathrm{O}_{2}$ treatment served as positive control. ${ }^{* * *} p<0.001$.

(G) Cell survival over time following LPS $(10 \mathrm{ng} / \mathrm{ml})$ and myelin $(10 \mu \mathrm{g} / \mathrm{ml})$ treatment. Evaluated by live imaging. Individual data points represent analyzed cells over $16 \mathrm{~h}$ treatment period $(n=8$ experiments, 261 cells). ${ }^{* *} \mathrm{p}<0.001$.

(H) Expression of the LXR target gene Abca1 in LPS $(10 \mathrm{ng} / \mathrm{ml})$ activated and non-activated wildtype BMDMs $(n=3)$ after $24 \mathrm{~h}$ squalene $(100 \mu \mathrm{M})$ or DMHCA $(10 \mu \mathrm{M})$ treatment normalized to untreated control (set to 1$)$. ${ }^{* * *} p<0.001,{ }^{* *} p<0.01,{ }^{*} p<0.05$.

(I) Expression of NOS2 in control and SQScKO BMDMs und high cholesterol (FCS) and lowcholesterol (LDS) conditions $(n=3)$ normalized to corresponding untreated control (set to 1$) .{ }^{* * *} p<$ $0.001,{ }^{*} p<0.05$.

(J) ROS measurement in control and SQScKO BMDMs $(n=3)$ normalized to untreated control (set to 1). ${ }^{* * *} p<0.001$.

(K) ROS measurement $(n=8)$ in control (left) and SQScKO BMDMs (right) stimulated with or without LPS and treated with or without squalene $(100 \mu \mathrm{M})$ normalized to untreated control (set to 1$).{ }^{* * *} \mathrm{p}$ $<0.001$.

S. Figure 6. LXR target gene upregulation and desmosterol accumulation in demyelinating mouse models, Related to figure 5

(A) Relative abundance of lanosterol, squalene and cholesterol normalized to cell number in isolated $\mathrm{CD} 11 \mathrm{~b}+$ myeloid cells during acute cuprizone, measured by lipid mass spectroscopy $(n=3-4){ }^{* * *} p$ $<0.001$.

(B) Relative abundance of lanosterol, lathosterol and desmosterol normalized to tissue weight and standard in lysolecithin induced spinal cord lesions $(n=4-5)$. ${ }^{* * *} p<0.001,{ }^{* *} p<0.01$.

(C) Relative abundance of sterol intermediates normalized to tissue weight and standard in corpus callosum during acute cuprizone of control and phagocyte SQScKO animals measured by lipid mass spectrometry $(n=3-5)$. Data normalized to non-disease controls (set to 1$)$. ${ }^{* * *} p<0.001,{ }^{* *} p<$ 0.01 .

(D) Relative abundance of sterol intermediates in spinal cord of groups in (B) during chronic EAE $(n=3-4)$ normalized to untreated controls (set to 1$)$. ${ }^{* * *} p<0.001,{ }^{* *} p<0.01$,

(E) Quantitative RT-PCR analysis determining expression of Abca1 and Apoe in isolated CD11b+ cells from chronic EAE (left panel), MBPcKO (middle panel) and lysolecithin (right panel) lesions $(n=3-4)$. Bars represent the means with individual data points normalized to isolated cells from control (set to 1). ${ }^{* * *} p<0.001,{ }^{* *} p<0.01,{ }^{*} p<0.05$.

(F) Relative abundance of sterol intermediates in spinal cord $(n=4)$ of squalene treated EAE animals normalized to untreated EAE controls (set to 1 ). ${ }^{* * *} p<0.001$.

S. Figure 7. Identity of inflammatory cell cluster in human MS scRNA-seq datasets and characterization of analyzed lesions, Related to figure $\mathbf{5}$

(A) Distribution of inflammatory cells from control and MS patients (left) and k-means clustering (right) of cells (resolution 0.1) on UMAP plot of merged external scRNA-seq datasets.

(B) Frequency distribution of cells from control and MS patients in each cluster.

(C) Expression of selected marker genes for myeloid cells (AIF1, CX3CR1), oligodendrocytes (PLP1) and immune cells (CD3, S100A4) in analyzed inflammatory cells of sc-RNAseq datasets.

(D) Expression of genes related to cholesterol export in analyzed inflammatory cells of sc-RNAseq datasets. 
(E) Histological characterization of isolated lesion from individual patients that were analyzed by mass spectrometry. Dotted line indicates lesion rim defined by myelin staining (LFB/PAS).Center activity (square) was determined by presents of phagocytic cells (KiM1P) (Scale 500 $\mu \mathrm{m})$.

(F) Tissue water ( $\mathrm{g} / \mathrm{g}$ dry tissue) of control white matter and MS lesion material. R: Lesion rim; $\mathrm{AC}$ : Active lesion center; IC: Inactive lesion center. ${ }^{* *} p<0.01,{ }^{*} p<0.05$.

(G) Relative abundance of cholesterol normalized to input tissue weight and standard (controls set to 1). Individual data points represent lesions (see $E$ and F) analyzed from MS patients $(n=4)$. ${ }^{* * *} p<0.001$.

S. Figure 8. Combination lipid based therapy, Related to figure 6

(A) Peripheral serum cholesterol profile of control and squalene treated lysolecithin lesion induced animals 14 days post injection ( $n=5-6)$. Measurement shows total cholesterol (TC), low density lipoprotein cholesterol (LDL) and high density lipoprotein cholesterol (HDL). Dotted line indicates values of non-disease controls.

(B) Squalene serum concentration measured by FPLC of control and squalene treated lysolecithin animals in $(A)(n=3) .{ }^{*} p<0.05$.

(C) Clinical score \pm SEM of control $(n=7)$, IFN $\beta-1 b(n=6)$, prophylactically squalene $(n=9)$ and prophylactically squalene in combination with IFN $\beta-1 b(n=8)$ treated EAE animals (see also figure $3 \mathrm{C}$ left).

(D) Mean clinical score (day 28) of EAE animals $(n=4)$ with or without cholesterol supplementation.

(E) Expression of the cholesterol export genes Dhcr24, Abca1 and Apoe in spinal cord tissue from cholesterol treated EAE animals and corresponding controls $(n=4)$ in $(F)$. ${ }^{* * *} p<0.001,{ }^{* *} p<0.01$, ${ }^{*} \mathrm{p}<0.05$.

(F) Clinical score \pm SEM of EAE control $(n=22)$, cholesterol $(n=19)$ and cholesterol in combination with IFN $\beta-1 b(n=19)$ treated EAE animals. Mice received cholesterol diet commencing at 14 days before immunization.

(G) Absolute number of CD4+ and CD8+ T cells, CD11b+/CD45+ myeloid cells per gram spinal cord determined by flow cytometry at chronic EAE (28 days) of animals in (D). ${ }^{* * *} p<0.001,{ }^{*} p<0.05$.

(H) mRNA expression of the inflammatory genes Tnf, I/2, I17, and Ifng in inflammatory infiltrate of groups in $(F) .{ }^{*} p<0.05$.

(I) Targeted expression profile of $\mathrm{CD} 11 \mathrm{~b}+$ isolated myeloid cells from EAE experimental groups in Figure $6 D(n=5)$. Heat map shows fold expression values of individual animals normalized to untreated EAE controls $(n=6$, set to 1$) .{ }^{* *} p<0.01,{ }^{*} p<0.05$

\section{S. Figure 9. Lipid therapy: Squalene treatment combination, Related to figure 2 and $\mathbf{6}$}

Working model of inflammatory response and remyelination efficiency in wildtype phagocytes (1), cholesterol export deficient phagocytes (2), phagocytes with squalene synthase inactivation (3) and phagocytes supplemented with squalene (4) during de- and remyelination. (1) Cholesterol rich myelin phagocytosis induces accumulation of the LXR ligand desmosterol leading to upregulation of the cholesterol efflux transporter ABCA1. LXR activation induces an anti-inflammatory switch enabling oligodendrocyte differentiation and remyelination. (2) Ablation of ABCA1 in phagocytes induces a mixed pro- and anti-inflammatory phenotype following phagocytosis, leading to foam cell formation and phagocyte pyroptosis, preventing orchestrated oligodendrocyte differentiation and remyelination. (3) Cholesterol synthesis ablation (SQScKO) in phagocytes prevents accumulation of the LXR ligand desmosterol following myelin phagocytosis, thereby blocking an anti-inflammatory switch and cholesterol export leading to foam cell formation hindering oligodendrocyte differentiation and remyelination. (4) Squalene supplementation in activated phagocytes supports desmosterol accumulation advancing anti-inflammatory potential, creating a permissive environment for oligodendrocyte differentiation and myelination. 


\section{Star Methods}

\section{KEY RESOURCES TABLE}

\begin{tabular}{|c|c|c|}
\hline REAGENT or RESOURCE & SOURCE & IDENTIFIER \\
\hline \multicolumn{3}{|l|}{ Antibodies } \\
\hline ABCA1 & Santa Cruz & Cat\#sc-58219 \\
\hline ACSA-2 & Miltenyi biotec & Cat \#130-097-679 \\
\hline CAII & Said Ghandour & N/A \\
\hline CD11b & Biolegend & Cat \#101201 \\
\hline CD11b & Miltenyi biotec & Cat \#130-093-636 \\
\hline CD31 & Miltenyi biotec & Cat \#130-091-935 \\
\hline CD3e & Biolegend & Cat \#100301 \\
\hline CD4 & Becton Dickinson & Cat \#553726 \\
\hline CD45.2 & Biolegend & Cat \#109805 \\
\hline CD8 & Becton Dickinson & Cat \#550281 \\
\hline GAPDH & Enzo & Cat \#ADI-CSA-335-E \\
\hline GFAP & Chemicon & Cat \#MAB3402 \\
\hline IBA1 & Wako & Cat \#019-19741 \\
\hline KiM1P & Prof. Dr. Radzun & N/A \\
\hline LAMP1 & BD Pharmingen & Cat \#09671D \\
\hline MAC3 & Pharmigen & Cat \#01781D \\
\hline MBP & Serotec & Cat \#MCA 4095 \\
\hline $\mathrm{O} 4$ & Miltenyi biotec & Cat \#130-096-670 \\
\hline OLIG2 & Prof. Stiles / Dr. Alberta & DF308 \\
\hline SMI31 & Covance & Cat \#SMI31P \\
\hline \multicolumn{3}{|c|}{ Chemicals, Peptides and Recombinant proteins } \\
\hline 2',7'-Dichlorofluorescein & Sigma-Aldrich & Cat \#D6665 \\
\hline 2',7'-Dichlorofluorescein & Sigma-Aldrich & Cat \#D6665 \\
\hline 4-hydroxytamoxifen & Sigma-Aldrich & Cat \#T176 \\
\hline Accustase & Innovative Cell Technologies & Cat \#AT104-500 \\
\hline BC theta & Max-Planck-Institute (MPI-EM) & N.A. \\
\hline Betaferon & BAYER & Cat \#04674936 \\
\hline Cholesterol & HEW & Cat \#60211 \\
\hline Corn oil & Sigma-Aldrich & Cat \#C8267 \\
\hline Cuprizone & Sigma-Aldrich & Cat \#14690 \\
\hline DMHCA & Avanti & Cat \#700125P \\
\hline Fluoromyelin & ThermoFisher Scientific & Cat \#F34651 \\
\hline Lipopolysaccharides (LPS) & Sigma-Aldrich & Cat \#L3012 \\
\hline Lymphocyte separation medium & PAA & Cat \#LSM1077 \\
\hline Lysolecithin & Sigma-Aldrich & Cat \#L4129 \\
\hline M. tuberculosis H37 Ra & $\mathrm{BD}$ & Cat \#231141 \\
\hline MOG35-55 & Max-Planck-Institute (MPI-EM) & N.A. \\
\hline Monastral blue & Sigma-Aldrich & Cat \#274011 \\
\hline PageRulerTM & ThermoFisher Scientific & Cat \#26619 \\
\hline Pertussis toxin & Sigma-Aldrich & Cat \#P7208 \\
\hline PhosSTOP ${ }^{\mathrm{TM}}$ & Roche & Cat \#4906845001 \\
\hline Squalene & Sigma-Aldrich & Cat \#S3626 \\
\hline Tamoxifen & Sigma-Aldrich & Cat \#T5648 \\
\hline Lipid depleted serum (LDS) & Biowest-bw & Cat \#S181L-500 \\
\hline \multicolumn{3}{|l|}{ Critical Commercial Assays } \\
\hline Adult Brain Dissociation Kit & Miltenyi biotec & Cat \#130-107-677 \\
\hline DAB Substrate Kit & Zymomed & Cat \#DAB057 \\
\hline LSAB®2 System-HRP & Dako & Cat \#K0609 \\
\hline Ovation Pico WTA System V2 & NuGEN & Cat \#3302 \\
\hline RNeasy Mini Kit & Qiagen & Cat \#74104 \\
\hline VECTASTAIN® Elite $₫$ ABC HRP Kit & VECTOR & Cat \#PK-6100 \\
\hline WST-1 cell proliferation Kit & Cayman & Cat \#10008883 \\
\hline
\end{tabular}




\begin{tabular}{|c|c|c|}
\hline \multicolumn{3}{|l|}{ Continued } \\
\hline \multicolumn{3}{|l|}{ Biological samples } \\
\hline Human Brain tissue & Netherland Brain Bank & https://www.brainbank.nl/ \\
\hline \multicolumn{3}{|c|}{ Experimental Models: Organisms/Strains } \\
\hline Plp1-CreERT2 & Max-Planck-Institute (MPI-EM) & (Leone et al., 2003) \\
\hline NG2-CreERT2 & Max-Planck-Institute (MPI-EM) & (Huang et al., 2014) \\
\hline GLAST-CreERT2 & Max-Planck-Institute (MPI-EM) & (Mori et al., 2006) \\
\hline CX3CR1-CreERT2 (Line 1) & Max-Planck-Institute (MPI-EM) & (Parkhurst et al., 2013) \\
\hline CX3CR1-CreERT2 (Line 2) & Max-Planck-Institute (MPI-EM) & (Yona et al., 2013) \\
\hline SLCO1c1-CreERT2 & Max-Planck-Institute (MPI-EM) & (Ridder et al., 2011) \\
\hline Aldh1l1-CreERT2 & Max-Planck-Institute (MPI-EM) & (Winchenbach et al., 2016) \\
\hline SQS $S^{\text {flox }}$ & Max-Planck-Institute (MPI-EM) & (Saher et al., 2005) \\
\hline Abca $1^{\text {flox fllox }}$ & Max-Planck-Institute (MPI-EM) & (Westerterp et al., 2012) \\
\hline Abcg $1^{\text {Hloxhllox }}$ & Max-Planck-Institute (MPI-EM) & (Westerterp et al., 2012) \\
\hline $\mathrm{MPB}^{\text {floxtlox }}$ & Max-Planck-Institute (MPI-EM) & (Meschkat et al., unpublished) \\
\hline Rosa26-tdTomato & Max-Planck-Institute (MPI-EM) & (Madisen et al., 2010) \\
\hline ApoEKO (Apoe -/-) & Max-Planck-Institute (MPI-EM) & (Piedrahita et al., 1992) \\
\hline \multicolumn{3}{|c|}{ Experimental Models: Cell lines } \\
\hline L929 & $\mathrm{N} / \mathrm{A}$ & (Garcia-Agudo et al., 2019) \\
\hline \multicolumn{3}{|l|}{ Oligonucleotides } \\
\hline Primer for RT-qPCR & This paper & $\mathrm{N} / \mathrm{A}$ \\
\hline \multicolumn{3}{|l|}{ Original source data } \\
\hline GSE118257 & https://www.ncbi.nlm.nih.gov & (Jakel et al., 2019) \\
\hline GSE124335 & https://www.ncbi.nlm.nih.gov & (Masuda et al., 2019) \\
\hline \multicolumn{3}{|c|}{ Software and Algorithms } \\
\hline Cellprofiler & (McQuin et al., 2018) & www.cellprofiler.org \\
\hline GraphPad Prism 6 & GraphPad Software, Inc. & https://www.graphpad.com/ \\
\hline ImageJ (Fiji) & (Schindelin et al., 2012) & https://imagej.nih.gov/ij/ \\
\hline InteractiVenn & (Heberle et al., 2015) & http://www.interactivenn.net/ \\
\hline LAS $X$ software & Leica-microsystems & https://www.leica-microsystems.com \\
\hline $\mathrm{R}$ & This paper & https://www.r-statistik.de \\
\hline R package ggplot2 v3.2.0 & (Wickham, 2009) & https://cran.r-project.org \\
\hline R package Seurat v3.1.0 & (Butler et al., 2018) & https://satijalab.org \\
\hline GSEA & Subramanian et al., 2005 & http://software.broadinstitute.org/gsea/index.jsp \\
\hline EnrichmentMap & (Merico et al., 2010) & http://apps.cytoscape.org/apps/enrichmentmap \\
\hline Cytoscape v3.7.1 & (Otasek et al., 2019) & https://cytoscape.org \\
\hline ZEN 2012 blue edition & Zeiss & https://www.zeiss.de \\
\hline
\end{tabular}

\section{LEAD CONTACT AND MATERIAL AVAILABILITY}

Further information and requests for resources and reagents should be directed to and will be fulfilled by the Lead Contact, Gesine Saher (Saher@em.mpg.de). This study did not generate new unique reagents.

\section{EXPERIMENTAL MODEL AND SUBJECT DETAILS}

\section{Animals}

All animal studies were performed in compliance with the animal policies of the Max Planck Institute of Experimental Medicine, and were approved by the German Federal State of Lower Saxony. Animals were group-housed (3-5 mice) with 12 hour dark/light cycle and had access to food and water ad libitum. Adult male and female C57BL/6N mice (8-10 weeks of age) or cholesterol synthesis mutants (8-10 weeks of age) were taken for all experiments. Male mice were subjected to cuprizone, EAE and lysolecithin experiments. Female mice were used for non-pathology induced experiments. Animals of same gender were randomly assigned to experimental groups (3-12 mice). Cholesterol synthesis mutants in this study were generated by crossbreeding cell specific Cre-driver lines (see key resources table) with mice harboring squalene synthase with two loxP sites flanking exon 5 , referred to as SQS-flox mice (SQScKO). 


\section{Human Specimens}

Human material of MS diagnosed and non-MS patients was purchased from Netherland Brain Bank after being approved by the Ethics Tissue Advisory Board, Nederlands Herseninstituut (Project no. 1206). Four MS patients and three non-MS control samples were analyzed (Table S2). Age of patients ranged between 47 to 61 , with a median age of 49 . Patients had mixed gender, five males and 2 females. Three patients could be identified as APOE33 allele carriers. Post mortem delay ranged between 5:15 to 11 hours. MS diagnosed patients were selected according to presence of histopathological characterized active, chronic active and chronic inactive lesions upon cryconservation. Non-MS patient material was selected by the absence of histopathological hallmarks. Frozen tissue received was histopathological examined to identify lesions for final analysis.

\section{Cell lines}

L929 mouse fibroblast cells were cultivated in complete media (DMEM, 10\% FCS, $1 \%$ Penicillin/Streptomycin) at $37^{\circ} \mathrm{C}$ under $5 \% \mathrm{CO}_{2}$. Cells were regularly passaged after reaching confluency using trypsin-EDTA $0.25 \%$. For conditioned media, supernatant was removed, sterile filtered and stored at $-20^{\circ} \mathrm{C}$.

\section{Primary cell culture \\ Myelinating cell cultures were established from embryonic day 13 (E13) mouse embryo (C57BL6/J Charles River) spinal cords as described previously (Bijland et al., 2019). Cells were cultured in differentiation media (low glucose DMEM, $10 \mathrm{mg} \mathrm{ml}^{-1}$ insulin, $10 \mathrm{ng} \mathrm{ml}^{-1}$ biotin, 50nM hydrocortisone, $0.5 \% \mathrm{~N} 1-\mathrm{mix}$ ) at $37^{\circ} \mathrm{C}$ under $5 \% \mathrm{CO}_{2}$. Bone marrow derived macrophages were isolated from femora bones of adult male mice. Cells were cultivated in L929 (20\%) conditioned media (DMEM, $1 \%$ Penicillin/Streptomycin, $10 \%$ FCS) at $37{ }^{\circ} \mathrm{C}$ with $7.5 \% \quad \mathrm{CO}_{2}$. All experiments involving animal specimens were performed according to the animal policies of the Max Planck Institute of Experimental Medicine, and were approved by the German Federal State of Lower Saxony.}

\section{METHOD DETAILS}

\section{Tamoxifen induced recombination}

Transgenic mice received tamoxifen either by oral administration, three times every second day at a concentration of $0.4 \mathrm{mg} / \mathrm{g}$ bodyweight dissolved in corn oil:ethanol (1:9) or by intraperitoneal injections on 5 consecutive days at a concentration of $75 \mu \mathrm{g} / \mathrm{g}$ body weight.

\section{Experimental autoimmune encephalomyelitis (EAE)}

MOG-EAE was induced in mice purchased from Charles River by immunizing subcutaneously with $200 \mathrm{mg}$ myelin oligodendrocyte glycoprotein peptide 35-55 (MOG35-55) in complete Freund's adjuvant (M. tuberculosis at $3.75 \mathrm{mg} \mathrm{ml}^{-1}$ ) and i.p. injection twice with $500 \mathrm{ng}$ pertussis toxin as described (Berghoff et al., 2017b). Animals were examined daily and scored for clinical signs of the disease. If disease did not start within 15 days after induction or the clinical score rose above 4, animals were excluded from the analysis. The clinical score was: 0 normal; 0.5 loss of tail tip tone; 1 loss of tail tone; 1.5 ataxia, mild walking deficits (slip off the grid); 2 mild hind limb weakness, severe gait ataxia, twist of the tail causes rotation of the whole body; 2.5 moderate hind limb weakness, cannot grip the grid with hind paw, but able to stay on a upright tilted grid; 3 mild paraparesis, falls down from a upright tiled grid; 3.5 paraparesis of hind limbs (legs strongly affected, but move clearly); 4 paralysis of hind limbs, weakness in forelimbs; 4.5 forelimbs paralyzed; 5 moribund/dead. Treatment commencing either two weeks before immunization was defined as prophylactic regimen or at the first appearance of EAE symptoms defined as therapeutic regimen. IFN $\beta 1 \mathrm{~b}$ (Bayer) was administrated by daily intraperitoneal (i.p.) injections $30.000 \mathrm{U}$ per animal. 


\section{Cuprizone}

Cuprizone pathology was induced by feeding mice with $0.2 \% \mathrm{w} / \mathrm{w}$ cuprizone (Sigma-Aldrich) in powder chow. Mice received cuprizone for 'demyelination' (5 weeks), 'acute remyelination' (6 weeks) and 'chronic remyelination' (12 weeks followed by 2 weeks normal chow) paradigms. For 'induced remyelination' experiments, mice were fed cuprizone in standard chow for 4 weeks, followed by cuprizone withdrawal and feeding mice standard chow with or without therapeutic dietary supplementation (e.g. Squalene). Mice were fed three times a week an exceeding amount of chow by dispenser. Food intake and animal weight was monitored. Age-matched untreated controls were fed standard powder chow.

\section{Focal spinal cord demyelinating lesions}

Focal spinal cord demyelinating lesions were induced under anaesthesia $(10 \mathrm{mg} / \mathrm{ml}$ ketamine and 1 $\mathrm{mg} / \mathrm{ml}$ xylazine) by stereotactic injection of $1 \mu \mathrm{l}$ lysolecithin (1\%, from egg yolk, alphalysophosphatidylcholine, Sigma-Aldrich) into the left and right ventro-lateral funiculus between Th3 and Th4 of 10-week old animals, as previously described (Cantuti-Castelvetri et al., 2018).Midline dorsal blood vessel was used as reference and capillary was positioned $0.5 \mathrm{~mm}$ left and right. The injection was performed at a rate of $150 \mathrm{nl} /$ minute. Injection site was marker by addition of Monastral blue (Sigma-Aldrich). 5 minutes after the delivery of the lysolecithin, the capillary was slowly retracted. At the day of injection, mice were randomly assigned to treatment groups.

\section{Therapeutic diets}

Therapeutic diets were prepared in powder chow (V1124 ssniff Spezialdiäten GmbH, Germany). Mice received either $0.5 \%$ squalene (oil), $5 \%$ cholesterol (powder) or $0.04 \%$ DMHCA (in corn oil) chow. Controls received either normal or in case of DMHCA, corn oil supplemented chow. Mice were fed three times a week an exceeding amount of chow by dispenser. Therapeutic dose was calculated according to mean daily food intake per gram mouse (Bachmanov et al., 2002).

\section{Serum Analysis}

Animals were anesthetized by intraperitoneal injection of Avertin (tribromethanol), blood was collected by cardiac puncture, and serum was prepared after $4 \mathrm{~h}$ clotting by centrifugation. Cholesterol measurements were done with the architectll system (Abbott Diagnostics). For squalene measurement, lipids were extracted from serum samples according to Bligh and Dyer (Bligh and Dyer, 1959). The chloroform phase containing the lipids was evaporated at $40^{\circ} \mathrm{C}$ in a vacuum concentrator (Qiagen) and extracted lipids redissolved and saponified in glass tubes with $0.5 \mathrm{M} \mathrm{KOH}$ in EtOH for 30 min. Non-saponifiable lipids were extracted with $n$-hexane. After evaporation of the $n$-hexane phase at $40{ }^{\circ} \mathrm{C}$ in a vacuum concentrator, non-saponifiable lipids were redissolved in $\mathrm{MetOH}$ and subjected to reverse phase HPLC (250/4 Nucleoshell RP C18 (Macherey and Nagel) connected to an Äkta FPLC (GE Healthcare), flow rate $0.25 \mathrm{ml} / \mathrm{min}$, liquid phase acetonitril:EtOH 70:30 (v/v), online UV detection at 205. Squalene retention and peak amplitude were related to injections of a range of concentration standards in $\mathrm{MetOH}$. Amplitudes were normalized to the neutral lipid recovery as assessed via online detection of fluorescence of dehydroergosterol (excitation $325 \mathrm{~nm}$, emission $375 \mathrm{~nm}$, Quantamaster (PTI/Horiba) that was spiked into the serum sample.

\section{Elevated Beam Test}

To assess bilater sensorimotor coordination that involves connecting white matter structures like the corpus callosum, elevated beam test (in-house made, MPI of experimental medicine) was applied. Mice were trained to run toward a hiding box on a beam (width $1.5 \mathrm{~cm}$ ) one week before testing. In one testing session number of slips in a defined $55 \mathrm{~cm}$ distance over three repeats was counted. Mean number of slips after three testing sessions was used as read out. 


\section{Flow cytometry}

Single-cell suspensions from spinal cords were obtained via mechanical dissociation on a cell strainer. Immune cells were separated over a two-phase Percoll-density gradient by centrifugation. Blood was collected by cardiac puncture in EDTA $(80 \mathrm{mM})$ and single-cell suspension was obtained by centrifugation over lymphocyte separation medium (PAA). Staining of $\alpha \beta T C R / C D 4+T$ cells, $\alpha \beta T C R / C D 8+T$ cells and CD45/CD11b cells (macrophages/microglia) was performed using the following antibodies in a 1:200 dilution: Anti-CD3e (clone 145-2C11), BioLegend; anti-CD4 (clone GK 1.5), BD; anti-CD8 (clone 53-6.7), BD; anti-CD8 (clone 53-6.7), BD; anti-CD11b (clone M1/70), BioLegend; anti-CD45.2 (clone 104), BioLegend. The addition of Calibrite APC beads (BD) allowed for cell quantification. Flow cytometry was performed using a FACSCalibur operated by Cell Quest software (Becton Dickinson).

\section{Magnetic cell isolation (MACS).}

Glial cells and neurons were isolated according to the adult brain dissociation protocol (Miltenyi biotec). Corpus callosum and/or cortex were isolated from Bregma +1.10 to -2.46 . Antibody labeling was done according to the Microbead kit protocols (Miltenyi biotec): Oligodendrocytes $(\mathrm{O} 4$, ); Astrocytes (ACSA-2, 130-097-679), Microglia (CD11b,), and Endothelial cells (CD31,). Neurons were isolated by negative selection. Purity of cell populations was routinely determined by qPCR on extracted and reverse transcribed RNA.

\section{Histochemistry}

Mice were anesthetized and perfused with $4 \%$ formaldehyde (PFA). In case of cuprizone treated animals, brain samples were cut at Bregma 1.58 to account for regional specificity of cuprizone pathology. Tissue was postfixed overnight, embedded in paraffin and cut into $5 \mathrm{~mm}$ sections (HMP 110, MICROM). Gallyas silver impregnation was done as described (Saher et al., 2012). Immunohistological staining's were done on deparaffinized sections followed by antigen-retrieval in sodium citrate buffer $(0.01 \mathrm{M}, \mathrm{pH} 6.0)$. For immunofluorescence detection, blocking was performed with serum free protein block (Dako). Primary antibodies were diluted in $2 \%$ bovine serum albumin (BSA)/PBS and incubated for $48 \mathrm{~h}$ followed by incubation with fluorophor coupled secondary antibodies. For chromogenic staining's, blocking of endogenous peroxidase activity with $3 \%$ hydrogen peroxide was performed followed by $20 \%$ goat serum (BSA/PBS)block and incubation with primary antibodies. Detection was carried out with the LSABß2 System-HRP (Dako, Hamburg, Germany) or the VECTASTAIN ${ }^{\circledR}$ Elite ${ }^{\circledR}$ ABC HRP Kit (Vector Labs). HRP substrate 3,30-Diaminobenzidine (DAB) was applied by using the DAB Zytomed Kit (Zytomed Systems $\mathrm{GmbH}$ ). Nuclear labeling was done by Haematoxylin stain followed by mounting (Eukitt). Samples were analyzed on an Axio Imager.Z1 (Zeiss) equipped with an AxioCam MRc3, x0.63 Camera Adaptor and the ZEN 2012 blue edition software using x10 objective (Plan Apochromat x10/0.45 M27) or x20 objective (Plan-Apochromat x20/0.8) and evaluated with Image J software. Quantification of areas (Gallyas, GFAP, MAC3) were done by applying semi-automated ImageJ software macro including thresholding (variable threshold in case of Gallyas and fixed threshold for antibody staining's) and colour deconvolution. Two to four sections per animal were analyzed.

\section{Multiple sclerosis tissue}

Frozen human material was cut on a cryotome to determine lesion localization and verify classification. Cryosections were fixed with 4\% PFA for 5 days. For LFB staining section were incubated in working solution over night at $60^{\circ} \mathrm{C}$ following differentiation with $0.05 \%$ lithium carbonate and $70 \%$ isopropyl alcohol. PAS staining was achieved by $1 \%$ periodic acid incubation for $5 \mathrm{~min}$. For KiM1p (Radzun et al., 1991) staining, antigen retrieval was performed with $10 \mathrm{mM}$ citric acid buffer (pH 6.0) followed by blocking of endogenous peroxidase with $0.3 \% \mathrm{H} 2 \mathrm{O} 2$ in PBS for $10 \mathrm{~min}$ at $4{ }^{\circ} \mathrm{C}$ and incubation and blocking with $10 \%$ FCS. First antibody was incubated overnight diluted in blocking buffer following biotinylated secondary antibody (GE Healthcare, Jackson ImmunoResearch and DCS Innovative Diagnostic Systems) incubation and peroxidase-conjugated avidin and DAB (Sigma-Aldrich) visualization. For sterol analysis by mass spectrometry, center and lesion rim as well as white matter of non-MS patients were dissected and stored at $-80^{\circ} \mathrm{C}$. 


\section{Electron microscopy}

Electron microscopic analysis was done as described (Berghoff et al., 2017b). 4\% PFA, 2.5\% Glutaraldehyde, $0.1 \mathrm{M}$ Phosphate buffer fixed sagittal sections were cut on a vibratome (Leica VT1200, $300 \mathrm{~mm})$. The corpus callosum with adjacent tissue $(-0.04 \mathrm{~mm}$ lateral) was punched with a $2 \mathrm{~mm}$ diameter punching tool and embedded in epon (EMTP, Leica) following region verification with methylene blue staining. Representative pictures (x12,000 magnification, TRS, Moorenweis) of uranyl acetate contrasted ultrathin sections were taken with the Zeiss EM900.

\section{Expression analyses}

For expression analyses of tissue samples, mice were killed by cervical dislocation. Samples were quickly cooled and region of interest prepared. RNA was extracted using RNeasy Mini (Qiagen). cDNA was synthesized with Superscript III (Invitrogen). Concentration and quality of RNA was evaluated using a NanoDrop spectrophotometer and RNA Nano (Agilent). RNA from MACS-purified cells was extracted using QIAshredder and RNeasy protocols (Qiagen). cDNA was amplified by Single Primer Isothermal Amplification (Ribo-SPIA® technology) using Ovation PicoSL WTA System V2 (NuGEN) following the manufactures protocol. Quantitative PCRs were done in triplicates on 384-well plates (Roche) using the GoTaq ${ }^{\circledR}$ qPCR Master Mix (Promega, A6002) and the LightCycler ${ }^{\circledR} 480$ Instrument (Roche). Background subtraction and thresholding was performed using the LightCycler ${ }^{\circledR} 480$ software (Roche). Expression values were normalized to the mean of housekeeping genes. Quantification was done by applying the $\Delta \Delta$ Ct method, normalized to experimental controls (set to 1). All primers (Table S1) were designed to fulfill optimal criteria e.g. primer length (18 $-22 \mathrm{bp}$ ), melting temperature $\left(52-58{ }^{\circ} \mathrm{C}\right)$, GC content $(40-60 \%)$, low number of repeats, amplicon length (<220 bp). All primers were intron-spanning.

\section{Myelin preparation}

Myelin was isolate from 12 weeks old mouse brains as described (Erwig et al., 2019). Brains were homogenized in $0.32 \mathrm{M}$ saccharose solution and added gently on top of $0.85 \mathrm{M}$ saccharose solution in an ultracentrifugation bucket and centrifuged for 30 minutes at $75.000 \times \mathrm{g}$. Interphase, with roughly purified myelin, was removed washed with water, and centrifuged for $15 \mathrm{~min}$ at 75,000 $\mathrm{x} \mathrm{g}$. Osmotic shock was performed by water incubation for 15 minutes followed by centrifugation for 15 minutes at $12,000 \times \mathrm{g}$. Pellet was resuspended in $0.35 \mathrm{M}$ saccharose solution added on top of $0.85 \mathrm{~m}$ saccharose solution and centrifuged for 30 minutes at 75,000 $\mathrm{g}$ g.Purified myelin was washed with water, and centrifuged for 15 minutes at $75,000 \times \mathrm{g}$. Supernatant was completely discarded, the pellet resuspended in 1xDPBS (without protease inhibitors, to not interfere with cells in culture), homogenized and stored at $-80^{\circ} \mathrm{C}$. Protein concentration of myelin was measured with Lowry DC Protein Assay (Bio-Rad).

\section{Myelinating Co-cultures}

Myelinating cell cultures were established from embryonic day 13 (E13) mouse embryo (C57BL6/J Charles River) spinal cords as described previously (Bijland et al., 2019). Briefly, spinal cords were dissected and dissociated in trypsin, then plated at 150,000 cells per coverslip (3 coverslips per 35 $\mathrm{mm}$ Petri dish) in serum-containing plating medium (50\% DMEM, 25\% HBSS, 25\% horse serum). Two hours later, differentiation medium (DMEM with $4.5 \mathrm{mg} / \mathrm{ml}$ glucose, hydrocortisone, insulin, biotin and N1) was added (Bottenstein and Sato, 1979). Two to three days later, half the medium was removed and replaced with differentiation medium plus HBSS (vehicle) or squalene (final concentration 100 $\mathrm{mM}$ ). Cells were fed every 2-3 days. Differentiation medium minus insulin was used from day in vitro (DIV) 12. On DIV 21 and DIV 30 (both +/- 2 days), cells were fixed for 10 minutes in $4 \%$ paraformaldehyde then stored in PBS until immunostained. Treatments were randomised across $8 \mathrm{x}$ $35 \mathrm{~mm}$ Petri dishes, and individual coverslips from 3 separate Petri dishes were fixed at each time point. Four independent experiments (embryos derived from 4 pregnant dams) were conducted. Cells were permeabilised in $0.5 \%$ trion $\mathrm{X}$ in PBS for 10 minutes, blocked in $10 \%$ goat serum for 30 minutes and incubated overnight at $4^{\circ} \mathrm{C}$ in primary antibody in blocking solution. Secondary antibody was applied in blocking solution for 1 hour after thorough washing. Cells were washed in PBS and briefly in 
tap water then coverslips were mounted on glass slides in Mowiol with DAPI. Axonal (SMI31) area and the area with myelin sheaths (MBP) of seven randomly chosen visual fields of myelinating co-cultures ( $x 20$ magnification) was measured by automated threshold with Fiji Software (SMI31 Otsu, MBP Triangle). Specimens were analyzed on an Axiophot observer.Z1 (Zeiss) equipped with an AxioCam MRm and the ZEN 2012 blue edition software and evaluated with Image $\mathrm{J}$ software.

\section{Bone marrow derived macrophages}

BMDMs were isolated from femora bones of adult mice. $5 \times 10^{5}$ cells were plated $10 \mathrm{~cm}$-diameter tissue culture dish in L929-CM (20\%). Cells were cultivated at $37{ }^{\circ} \mathrm{C}$ with $7.5 \% \mathrm{CO} 2 \mathrm{v} / \mathrm{v}$. In vitro recombination was achieved by 4-hydroxytamoxifen (Sigma-Aldrich) administration at a final dose of 4 $\mu \mathrm{M}$ for 10 days starting $24 \mathrm{~h}$ after plating. For experimental seeding, cells were harvested following Accutase (Innovative Cell Technologies Inc.) induced detachment. Cells were either seeded into cell culture grade plasticware, onto Poly-L-lysine (PLL) coated, $\mathrm{HCl}$-washed $1.5 \# 12 \mathrm{~mm}$ diameter glass coverslips, or into PLL coated glass bottom chamberslides (Ibidi) for live imaging. Cells were seeded (6-well: $250 \mathrm{k}$ cells; 96 - well: $18 \mathrm{k}$ cells; $12 \mathrm{~mm}$ coverslip: $18 \mathrm{k}$ cells; 8 -chamberslide: $18 \mathrm{k}$ cells) in cultivation media. Media was changed to different experimental condition (DMEM, 1\% P/S, 10\% Fetal calf serum or lipid depleted serum) after $24 \mathrm{~h}$. For activation, cells were treated with LPS (10 $\mathrm{ng} / \mathrm{mL}$ ) and/or myelin $(10 \mu \mathrm{g} / \mathrm{mL})$ if not otherwise stated. Squalene $(100 \mu \mathrm{M})$ was added $24 \mathrm{~h}$ before activation.

\section{In vitro phagocytosis}

In vitro phagocytosis was analyzed by FluoroMyelin Green pre-labeling of myelin and fixation with $4 \%$ PFA. For fluorescence staining's, cell were permeabilized for 5 minutes with $0.1 \%$ TritonX-100, blocked for 30 minutes with $4 \% \mathrm{HS}, 2 \% \mathrm{BSA}$ in PBS and incubate with primary antibody (IBA1, LAMP1) overnight. Cells were incubated with secondary antibody in blocking solution for $1 \mathrm{~h}$ following $1 \mathrm{~min}$ nuclear DAPI labeling and mounting on slides. Fluoromyelin positive accumulations were quantified using CellProfiler software defining cells by DAPI and IBA1 positive staining. Live imaging was performed on a Axio Imager.Z1 (Zeiss) equipped with an AxioCam MRc3, x0.63 Camera Adaptor and the ZEN 2012 blue edition software using $\times 20$ objective (Plan-Apochromat $\times 20 / 0.8$ ) at $37{ }^{\circ} \mathrm{C}$ and $5 \% \mathrm{CO} 2 \mathrm{v} / \mathrm{v}$. Cell were image over $16 \mathrm{~h}$ with a frame rate of 5-8 minutes and Definite Focus (Zeiss) to maintain focus level. Analysis was performed with Image $\mathrm{J}$ software to determine survival rate.

\section{Cholesterol staining}

To visualize cholesterol-rich cellular domains $B C$ theta live cell staining was performed. Cell were washed on ice (DPBS), blocked for 10 minutes with $1 \%$ BSA in PBS and incubated with BC Theta $(0.015 \mathrm{mg} / \mathrm{mL})$ for 30 minutes following fixation with $4 \%$ PFA. Cells were imaged with a Leica SP8 Lightning microscope and LAS $X$ software at $63 x$ magnification and stacks were processed as maximum intensity projection.

\section{Measurement of Reactive Oxygen Species (ROS)}

ROS production was measured by 2',7' dichlorofluoresceindiacetate (DCFDA) detection according to published protocols (Wang and Roper, 2014) with minor modifications. For measurement, cells were incubated for 25 minutes at $37^{\circ} \mathrm{C}$ with $5 \mu \mathrm{M}$ DCFDA in 1x DPBS with $4.5 \mathrm{~g} / \mathrm{L}$ D-glucose. DPBS was aspirated and cells were incubated for 10 - 20 minutes in DMEM (without PhenolRed, $4.5 \mathrm{~g} / \mathrm{L}$ Dglucose and $3.7 \mathrm{~g} / \mathrm{L}$ sodium bicarbonate), to allow deacetylation and oxidation. 96-well flat bottom plates were then measured with Eon plate reader (Biotek) at excitation / emission of $495 \mathrm{~nm} / 529 \mathrm{~nm}$. Relative ROS production was calculated after blank substraction and normalization to untreated controls (set to 1). 


\section{Cell survival and proliferation}

WST-1 cell proliferation assay was performed according to the manufacturers protocol (Cayman Chemicals). Therefore, Electron Mediator Solution was mixed with WST-1 Developer Reagent immediately before use. After incubation under experiment conditions, media in wells was replaced with DMEM (without PhenolRed, with $4.5 \mathrm{~g} / \mathrm{L}$ D-glucose and $3.7 \mathrm{~g} / \mathrm{L}$ sodium bicarbonate) containing WST-1 mixture (100 $\mu \mathrm{L}$ DMEM $+10 \mu \mathrm{L}$ WST-1 mixture per 96-well). Cells were incubated for two hours at $37{ }^{\circ} \mathrm{C}$. Absorbance of formazan was measured at $450 \mathrm{~nm}$. As positive control, cell were treated with $\mathrm{H}_{2} \mathrm{O}_{2}(20 \mathrm{mM})$.

\section{Chromatographic-mass spectrometry (GC-MS)}

For lipid mass spectrometry, samples were lyophilized as described previously (Berghoff et al., 2017a).In case of cuprizone the demyelinated corpus callosum was isolated. For EAE lumbar spinal cord tissue was used. To determine sterol abundance of in vivo isolated cells, 4-5 animals were pooled for each sample. In vitro analysis was done by using 700.000 to 3500.000 cells following removal of supernatant by centrifugation. For lyophilization, samples were exposed to a shelf temperature of $-56{ }^{\circ} \mathrm{C}$ for $24 \mathrm{~h}$ under vacuum of $0.2 \mathrm{mBar}$ (Christ LMC-1 BETA 1-16). Samples were weighed for calculation of water content and normalization. Sterols were measured by gaschromatographic-mass spectrometry GC-MS after extraction and derivatization as previously described (Rotsch et al., 2017). Lyophilized tissue was ground to a fine powder using a beatmill (Retsch, Haan, Germany) and glass beads (5 mm, Carl Roth, Karlsruhe Germany) and extracted with $500 \mu \mathrm{l}$ methanol:chloroform:water 129:50:25 [v/v/v]. Phase separation was induced by addition of the internal standard $(250 \mu \mathrm{l} 5 \mu \mathrm{g} / \mathrm{ml}$ allo-inositol in water). Sterols were extracted from the organic phase by adding $800 \mu \mathrm{l}$ of MTBE:MeOH (v/v) and $200 \mu \mathrm{l}$ of $0.9 \% \mathrm{NaCl}$ and $40 \mu \mathrm{l} 17: 0$ fatty acid $(0.25 \mathrm{mg} / \mathrm{ml}$ in chloroform). Each sample was measured twice. One time, $10 \mu \mathrm{l}$ of the organic phase was used and run later with a 1:50 split to measure cholesterol. The second time, $50 \mu$ of the organic phase was used and run later with a 1:10 split to measure other sterols. All samples were dried by N2 stream and redissolved in $15 \mu$ pyridine. $30 \mu$ of MSTFA was added followed by an incubation time of 2-10 h. Sterols were measured by GC-MS using the same set-up as described for small metabolites except for the temperature gradient, which was run at $180^{\circ} \mathrm{C}$ for $1 \mathrm{~min}, 180-320^{\circ} \mathrm{C}$ at $5 \mathrm{~K} / \mathrm{min}, 320^{\circ} \mathrm{C}$ for 5 min. The abundance of sterols was compared based on their TIC.

\section{Western Blot}

Animals were sacrificed by cervical dislocation, corpus callosum samples were dissected on ice and snap frozen on dry ice. For protein analyses tissue samples were homogenized in pre-cooled sucrose buffer containing protease and phosphatase inhibitors (PhosSTOPTM, Roche) using preccellys 24 (Bertin technologies) at 5.000rpm for $3 \times 10$ seconds. Lysates were centrifuged for $5 \mathrm{~min}$ at $13.000 \mathrm{rpm}$ to remove nuclei. Protein concentrations were measured with Lowry method (LOWRY et al., 1951) and Bio-Rad DC Protein Assay kit following manufacturer's instructions. Protein separation was performed by SDS-PAGE (12\%) using Mini-PROTEANTM Handcast System (BIO-RAD). Samples were diluted in $1 \mathrm{x}$ SDS sample buffer and $8 \%$ 2-Mercaptoethanol or Dithiothreitol $(0,5 \mathrm{M})$. Protein lysates were denaturated on a shaker for $10 \mathrm{~min}$ at $40^{\circ} \mathrm{C}$ or $70^{\circ} \mathrm{C}$ corresponding to the used antibody. 10-25 $\mathrm{\mu g}$ sample was loaded at as well as $3 \mu \mathrm{l}$ of pre-stained protein ladder (PageRulerTM, ThermoFisher Scientific). Proteins were transferred to PVDF membranes (GE Healthcare, 10600023) using a tank blot system (XCell SureLockTM Mini-Cell Electrophoresis System, ThermoFisher Scientific). Membranes were incubated in 1x TBS containing 0.05\% Tween-20 (Promega, H5151, 1x TBS-T) and $5 \% \mathrm{w} / \mathrm{v}$ skim milk powder (blocking buffer) for $60 \mathrm{~min}$ under constant shaking at RT. Primary Antibodies were diluted in blocking buffer and incubated overnight at $4^{\circ} \mathrm{Cunder}$ constant shaking. Immunodetection was archieved by horseradish peroxidase (HRP)-coupled secondary antibodies diluted in blocking buffer for $1 \mathrm{~h}$ at RT following incubation in Enhanced Chemiluminescence solution (Western Lightning ${ }^{\circledR}$ Plus, Perkin Elmer) and signal detection using the ChemoStar ECL Imager HR 6.0 (Intas). Relative protein abundance (controls set to 1) was calculated using Image J Gel analyzer and normalized to loading controls (Actin or GAPDH). 
Human single-cell/nuclei transcriptome sequencing datasets

Human single-cell/nuclei RNA sequencing profile were obtained from two available datasets, GSE118257 and GSE124335, and analyzed by R package Seurat v3.1.0 (Butler et al., 2018; Stuart et al., 2019). Gene counts from inflammatory cell nucleus were recovered from GSE118257 and merged with GSE124335 by applying Canonical Correlation Analysis (CCA). The resulting four major clusters were detected under resolution 0.1 and two dimensional visualization was performed by UMAP (Leland Mclnnes, 2018). Proportion and average gene expression level of cells in each cluster were calculated and then visualized using R package ggplot2 v3.2.0 (Wickham, 2009). Cells in cluster 2 (annotated as cholesterol export cluster) were compared to cells in cluster 1 to detect Differentially Expressed Genes (DEGs). Gene ontology enrichment analysis and visualization of DEGs were performed by GSEA (Mootha et al., 2003; Subramanian et al., 2005) and EnrichmentMap (Merico et al., 2010) in Cytoscape v3.7.1 (Shannon et al., 2003), respectively.

\section{QUANTIFICATION AND STATISTICAL ANALYSIS}

Number of animals and in vitro replicates for each experiment is shown in the figure legends. No inclusion or exclusion criteria were used if not otherwise stated. Studies were conducted blinded to investigators and/or formally randomized. Data are expressed as mean ISEM unless otherwise indicated. For statistical analysis, unpaired two-sided Student's t-test, one-way ANOVA with Tukey correction for multiple comparisons, two-way ANOVA followed by Tukey's post hoc test to compare interactions or Holm-Sidak method when appropriate, was applied. Normality was checked using the Kolmogorov-Smirnov test. Nonparametric Mann-Whitney test was used when data did not pass the normality test. A value of $p \leq 0.05$ was considered statistically significant. Data analysis was performed using GraphPad Prism Software Version 6 (GraphPad).

\section{DATA AND CODE AVAILABILITY}

This study did not generate datasets or codes. Originial data for figures in this study are available through the Lead Contact, Gesine Saher (Saher@em.mpg.de). Original Analyzed Human singlecell/nuclei RNA sequencing data in this paper is available on NCBI GEO DataSets: GSE118257 and GSE124335.

\section{Supplemental Information}

\section{Supplemental Table 1: List of primer sequences, Related to figure 1-6}

Supplemental Table 2: Patient characteristics, Related to figure 5

Supplemental Table 3: Mean expression level of inflammatory cell clusters, Related to figure 5

Supplemental Video 1: Cholesterol synthesis ablation (SQScKO) and cholesterol export deficiency (ABC1cKO) in BMDMs induces pyroptosis like cell death, Related to figure 2 


\section{References}

Abbott, N.J., Patabendige, A.A., Dolman, D.E., Yusof, S.R., and Begley, D.J. (2010). Structure and function of the blood-brain barrier. Neurobiology of disease 37, 13-25. Alizadeh, A., and Karimi-Abdolrezaee, S. (2016). Microenvironmental regulation of oligodendrocyte replacement and remyelination in spinal cord injury. The Journal of physiology 594, 3539-3552.

Araldi, E., Fernandez-Fuertes, M., Canfran-Duque, A., Tang, W., Cline, G.W., MadrigalMatute, J., Pober, J.S., Lasuncion, M.A., Wu, D., Fernandez-Hernando, C., et al. (2017). Lanosterol Modulates TLR4-Mediated Innate Immune Responses in Macrophages. Cell reports $19,2743-2755$.

Bachmanov, A.A., Reed, D.R., Beauchamp, G.K., and Tordoff, M.G. (2002). Food intake, water intake, and drinking spout side preference of 28 mouse strains. Behavior genetics 32 , 435-443.

Beltrán, G., Bucheli, M.E., Aguilera, M.P., Belaj, A., and Jimenez, A. (2016). Squalene in virgin olive oil: Screening of variability in olive cultivars. European Journal of Lipid Science and Technology.

Benardais, K., Kotsiari, A., Skuljec, J., Koutsoudaki, P.N., Gudi, V., Singh, V., Vulinovic, F., Skripuletz, T., and Stangel, M. (2013). Cuprizone [bis(cyclohexylidenehydrazide)] is selectively toxic for mature oligodendrocytes. Neurotoxicity research 24, 244-250.

Bennett, J., Basivireddy, J., Kollar, A., Biron, K.E., Reickmann, P., Jefferies, W.A., and McQuaid, S. (2010). Blood-brain barrier disruption and enhanced vascular permeability in the multiple sclerosis model EAE. Journal of neuroimmunology 229, 180-191.

Berghoff, S.A., Duking, T., Spieth, L., Winchenbach, J., Stumpf, S.K., Gerndt, N., Kusch, K., Ruhwedel, T., Mobius, W., and Saher, G. (2017a). Blood-brain barrier hyperpermeability precedes demyelination in the cuprizone model. Acta neuropathologica communications 5 , 94.

Berghoff, S.A., Gerndt, N., Winchenbach, J., Stumpf, S.K., Hosang, L., Odoardi, F., Ruhwedel, T., Böhler, C., Barrette, B., Stassart, R., et al. (2017b). Dietary cholesterol promotes repair of demyelinated lesions in the adult brain. Nature communications 8, 14241. Bijland, S., Thomson, G., Euston, M., Michail, K., Thummler, K., Mucklisch, S., Crawford, C.L., Barnett, S.C., McLaughlin, M., Anderson, T.J., et al. (2019). An in vitro model for studying CNS white matter: functional properties and experimental approaches.

F1000Research 8, 117.

Bligh, E.G., and Dyer, W.J. (1959). A rapid method of total lipid extraction and purification. Canadian journal of biochemistry and physiology 37, 911-917.

Bogie, J.F., Timmermans, S., Huynh-Thu, V.A., Irrthum, A., Smeets, H.J., Gustafsson, J.A., Steffensen, K.R., Mulder, M., Stinissen, P., Hellings, N., et al. (2012). Myelin-derived lipids modulate macrophage activity by liver $\mathrm{X}$ receptor activation. PloS one 7, e44998.

Bottenstein, J.E., and Sato, G.H. (1979). Growth of a rat neuroblastoma cell line in serumfree supplemented medium. Proceedings of the National Academy of Sciences of the United States of America 76, 514-517.

Boven, L.A., Van Meurs, M., Van Zwam, M., Wierenga-Wolf, A., Hintzen, R.Q., Boot, R.G., Aerts, J.M., Amor, S., Nieuwenhuis, E.E., and Laman, J.D. (2006). Myelin-laden macrophages are anti-inflammatory, consistent with foam cells in multiple sclerosis. Brain : a journal of neurology 129, 517-526.

Butler, A., Hoffman, P., Smibert, P., Papalexi, E., and Satija, R. (2018). Integrating single-cell transcriptomic data across different conditions, technologies, and species. Nature biotechnology $36,411-420$.

Cantuti-Castelvetri, L., Fitzner, D., Bosch-Queralt, M., Weil, M.T., Su, M., Sen, P., Ruhwedel, T., Mitkovski, M., Trendelenburg, G., Lutjohann, D., et al. (2018). Defective cholesterol clearance limits remyelination in the aged central nervous system. Science 359, 684-688. Chan, P., Tomlinson, B., Lee, C.B., and Lee, Y.S. (1996). Effectiveness and safety of lowdose pravastatin and squalene, alone and in combination, in elderly patients with hypercholesterolemia. Journal of clinical pharmacology 36, 422-427.

Dietschy, J.M. (2009). Central nervous system: cholesterol turnover, brain development and neurodegeneration. Biological chemistry 390, 287-293. 
Duewell, P., Kono, H., Rayner, K.J., Sirois, C.M., Vladimer, G., Bauernfeind, F.G., Abela, G.S., Franchi, L., Nunez, G., Schnurr, M., et al. (2010). NLRP3 inflammasomes are required for atherogenesis and activated by cholesterol crystals. Nature 464, 1357-1361.

Erwig, M.S., Patzig, J., Steyer, A.M., Dibaj, P., Heilmann, M., Heilmann, I., Jung, R.B., Kusch, K., Mobius, W., Jahn, O., et al. (2019). Anillin facilitates septin assembly to prevent pathological outfoldings of central nervous system myelin. eLife 8 .

Franklin, R.J. (2002). Why does remyelination fail in multiple sclerosis? Nature reviews. Neuroscience 3, 705-714.

Franklin, R.J., and Ffrench-Constant, C. (2008). Remyelination in the CNS: from biology to therapy. Nature reviews. Neuroscience 9, 839-855.

Gabas-Rivera, C., Barranquero, C., Martinez-Beamonte, R., Navarro, M.A., Surra, J.C., and Osada, J. (2014). Dietary squalene increases high density lipoprotein-cholesterol and paraoxonase 1 and decreases oxidative stress in mice. PloS one 9, e104224.

Garcia-Agudo, L.F., Janova, H., Sendler, L.E., Arinrad, S., Steixner, A.A., Hassouna, I., Balmuth, E., Ronnenberg, A., Schopf, N., van der Flier, F.J., et al. (2019). Genetically induced brain inflammation by $\mathrm{Cnp}$ deletion transiently benefits from microglia depletion. FASEB journal : official publication of the Federation of American Societies for Experimental Biology 33, 8634-8647.

Goldberg, J., Daniel, M., van Heuvel, Y., Victor, M., Beyer, C., Clarner, T., and Kipp, M. (2013). Short-term cuprizone feeding induces selective amino acid deprivation with concomitant activation of an integrated stress response in oligodendrocytes. Cellular and molecular neurobiology 33, 1087-1098.

Goodman, D.S. (1964). Squalene in Human and Rat Blood Plasma. The Journal of clinical investigation 43, 1480-1485.

Grajchen, E., Hendriks, J.J.A., and Bogie, J.F.J. (2018). The physiology of foamy phagocytes in multiple sclerosis. Acta neuropathologica communications 6, 124.

Gylling, H., and Miettinen, T.A. (1994). Postabsorptive metabolism of dietary squalene. Atherosclerosis 106, 169-178.

Heberle, H., Meirelles, G.V., da Silva, F.R., Telles, G.P., and Minghim, R. (2015). InteractiVenn: a web-based tool for the analysis of sets through Venn diagrams. BMC bioinformatics 16, 169 .

Hendrickx, D.A.E., van Scheppingen, J., van der Poel, M., Bossers, K., Schuurman, K.G., van Eden, C.G., Hol, E.M., Hamann, J., and Huitinga, I. (2017). Gene Expression Profiling of Multiple Sclerosis Pathology Identifies Early Patterns of Demyelination Surrounding Chronic Active Lesions. Frontiers in immunology 8, 1810.

Hien, H.T.M., Ha, N.C., Thom, L.T., and Hong, D.D. (2017). Squalene promotes cholesterol homeostasis in macrophage and hepatocyte cells via activation of liver $X$ receptor (LXR) alpha and beta. Biotechnology letters 39, 1101-1107.

Hong, C., and Tontonoz, P. (2014). Liver X receptors in lipid metabolism: opportunities for drug discovery. Nature reviews. Drug discovery 13, 433-444.

Huang, W., Zhao, N., Bai, X., Karram, K., Trotter, J., Goebbels, S., Scheller, A., and Kirchhoff, F. (2014). Novel NG2-CreERT2 knock-in mice demonstrate heterogeneous differentiation potential of NG2 glia during development. Glia 62, 896-913.

Hwang, B., Lee, J.H., and Bang, D. (2018). Single-cell RNA sequencing technologies and bioinformatics pipelines. Experimental \& molecular medicine 50, 96.

Itoh, N., Itoh, Y., Tassoni, A., Ren, E., Kaito, M., Ohno, A., Ao, Y., Farkhondeh, V., Johnsonbaugh, H., Burda, J., et al. (2018). Cell-specific and region-specific transcriptomics in the multiple sclerosis model: Focus on astrocytes. Proceedings of the National Academy of Sciences of the United States of America 115, E302-E309.

Jakel, S., Agirre, E., Mendanha Falcao, A., van Bruggen, D., Lee, K.W., Knuesel, I., Malhotra, D., Ffrench-Constant, C., Williams, A., and Castelo-Branco, G. (2019). Altered human oligodendrocyte heterogeneity in multiple sclerosis. Nature 566, 543-547.

Jamroz-Wisniewska, A., Wojcicka, G., Horoszewicz, K., and Beltowski, J. (2007). Liver X receptors (LXRs). Part II: non-lipid effects, role in pathology, and therapeutic implications. Postepy higieny i medycyny doswiadczalnej $61,760-785$. 
Jang, J., Park, S., Jin Hur, H., Cho, H.J., Hwang, I., Pyo Kang, Y., Im, I., Lee, H., Lee, E., Yang, W., et al. (2016). 25-hydroxycholesterol contributes to cerebral inflammation of Xlinked adrenoleukodystrophy through activation of the NLRP3 inflammasome. Nature communications 7, 13129.

Jurevics, H., and Morell, P. (1995). Cholesterol for synthesis of myelin is made locally, not imported into brain. Journal of neurochemistry 64, 895-901.

Katz Sand, I. (2018). The Role of Diet in Multiple Sclerosis: Mechanistic Connections and Current Evidence. Current nutrition reports 7, 150-160.

Kim, K.W., Vallon-Eberhard, A., Zigmond, E., Farache, J., Shezen, E., Shakhar, G., Ludwig, A., Lira, S.A., and Jung, S. (2011). In vivo structure/function and expression analysis of the CX3C chemokine fractalkine. Blood 118, e156-167.

Kipp, M., Clarner, T., Dang, J., Copray, S., and Beyer, C. (2009). The cuprizone animal model: new insights into an old story. Acta neuropathologica 118, 723-736.

Kocur, M., Schneider, R., Pulm, A.K., Bauer, J., Kropp, S., Gliem, M., Ingwersen, J., Goebels, N., Alferink, J., Prozorovski, T., et al. (2015). IFNbeta secreted by microglia mediates clearance of myelin debris in CNS autoimmunity. Acta neuropathologica communications 3, 20.

Lampron, A., Larochelle, A., Laflamme, N., Prefontaine, P., Plante, M.M., Sanchez, M.G., Yong, V.W., Stys, P.K., Tremblay, M.E., and Rivest, S. (2015). Inefficient clearance of myelin debris by microglia impairs remyelinating processes. The Journal of experimental medicine 212, 481-495.

Lane-Donovan, C., Philips, G.T., and Herz, J. (2014). More than cholesterol transporters: lipoprotein receptors in CNS function and neurodegeneration. Neuron 83, 771-787.

Lassmann, H., and Bradl, M. (2017). Multiple sclerosis: experimental models and reality. Acta neuropathologica 133, 223-244.

Lavrnja, I., Smiljanic, K., Savic, D., Mladenovic-Djordjevic, A., Tesovic, K., Kanazir, S., and Pekovic, S. (2017). Expression profiles of cholesterol metabolism-related genes are altered during development of experimental autoimmune encephalomyelitis in the rat spinal cord. Scientific reports 7, 2702.

Leland Mclnnes, J.H., James Melville (2018). UMAP: Uniform Manifold Approximation and Projection for Dimension Reduction. arXiv.org 1802.03426.

Leone, D.P., Genoud, S., Atanasoski, S., Grausenburger, R., Berger, P., Metzger, D., Macklin, W.B., Chambon, P., and Suter, U. (2003). Tamoxifen-inducible glia-specific Cre mice for somatic mutagenesis in oligodendrocytes and Schwann cells. Molecular and cellular neurosciences 22, 430-440.

Lloyd, A.F., Davies, C.L., Holloway, R.K., Labrak, Y., Ireland, G., Carradori, D., Dillenburg, A., Borger, E., Soong, D., Richardson, J.C., et al. (2019). Central nervous system regeneration is driven by microglia necroptosis and repopulation. Nature neuroscience 22 , 1046-1052.

Lodygin, D., Odoardi, F., Schlager, C., Korner, H., Kitz, A., Nosov, M., van den Brandt, J., Reichardt, H.M., Haberl, M., and Flugel, A. (2013). A combination of fluorescent NFAT and H2B sensors uncovers dynamics of T cell activation in real time during CNS autoimmunity. Nature medicine 19, 784-790.

Loike, J.D., Shabtai, D.Y., Neuhut, R., Malitzky, S., Lu, E., Husemann, J., Goldberg, I.J., and Silverstein, S.C. (2004). Statin inhibition of Fc receptor-mediated phagocytosis by macrophages is modulated by cell activation and cholesterol. Arteriosclerosis, thrombosis, and vascular biology 24, 2051-2056.

Luu, W., Zerenturk, E.J., Kristiana, I., Bucknall, M.P., Sharpe, L.J., and Brown, A.J. (2014). Signaling regulates activity of DHCR24, the final enzyme in cholesterol synthesis. Journal of lipid research 55, 410-420.

Madisen, L., Zwingman, T.A., Sunkin, S.M., Oh, S.W., Zariwala, H.A., Gu, H., Ng, L.L., Palmiter, R.D., Hawrylycz, M.J., Jones, A.R., et al. (2010). A robust and high-throughput Cre reporting and characterization system for the whole mouse brain. Nature neuroscience 13 , 133-140. 
Mailleux, J., Vanmierlo, T., Bogie, J.F., Wouters, E., Lutjohann, D., Hendriks, J.J., and van Horssen, J. (2018). Active liver $X$ receptor signaling in phagocytes in multiple sclerosis lesions. Multiple sclerosis 24, 279-289.

Masuda, T., Sankowski, R., Staszewski, O., Bottcher, C., Amann, L., Sagar, Scheiwe, C., Nessler, S., Kunz, P., van Loo, G., et al. (2019). Spatial and temporal heterogeneity of mouse and human microglia at single-cell resolution. Nature 566, 388-392.

Matsushima, G.K., and Morell, P. (2001). The neurotoxicant, cuprizone, as a model to study demyelination and remyelination in the central nervous system. Brain pathology 11, 107-116. McQuin, C., Goodman, A., Chernyshev, V., Kamentsky, L., Cimini, B.A., Karhohs, K.W., Doan, M., Ding, L., Rafelski, S.M., Thirstrup, D., et al. (2018). CellProfiler 3.0: Nextgeneration image processing for biology. PLoS biology 16, e2005970.

Merico, D., Isserlin, R., Stueker, O., Emili, A., and Bader, G.D. (2010). Enrichment map: a network-based method for gene-set enrichment visualization and interpretation. PloS one 5 , e13984.

Mootha, V.K., Lindgren, C.M., Eriksson, K.F., Subramanian, A., Sihag, S., Lehar, J., Puigserver, P., Carlsson, E., Ridderstrale, M., Laurila, E., et al. (2003). PGC-1alpharesponsive genes involved in oxidative phosphorylation are coordinately downregulated in human diabetes. Nature genetics 34, 267-273.

Mori, T., Tanaka, K., Buffo, A., Wurst, W., Kuhn, R., and Gotz, M. (2006). Inducible gene deletion in astroglia and radial glia--a valuable tool for functional and lineage analysis. Glia 54, 21-34.

Munji, R.N., Soung, A.L., Weiner, G.A., Sohet, F., Semple, B.D., Trivedi, A., Gimlin, K., Kotoda, M., Korai, M., Aydin, S., et al. (2019). Profiling the mouse brain endothelial transcriptome in health and disease models reveals a core blood-brain barrier dysfunction module. Nature neuroscience 22, 1892-1902.

Muse, E.D., Yu, S., Edillor, C.R., Tao, J., Spann, N.J., Troutman, T.D., Seidman, J.S., Henke, A., Roland, J.T., Ozeki, K.A., et al. (2018). Cell-specific discrimination of desmosterol and desmosterol mimetics confers selective regulation of LXR and SREBP in macrophages. Proceedings of the National Academy of Sciences of the United States of America 115, E4680-E4689.

Nogueras, L., Gonzalo, H., Jove, M., Sol, J., Gil-Sanchez, A., Hervas, J.V., Valcheva, P., Gonzalez-Mingot, C., Solana, M.J., Peralta, S., et al. (2019). Lipid profile of cerebrospinal fluid in multiple sclerosis patients: a potential tool for diagnosis. Scientific reports 9, 11313. Nordskog, B.K., Reagan, J.W., Jr., and St Clair, R.W. (1999). Sterol synthesis is upregulated in cholesterol-loaded pigeon macrophages during induction of cholesterol efflux. Journal of lipid research 40, 1806-1817.

Oiknine, J., and Aviram, M. (1992). Increased susceptibility to activation and increased uptake of low density lipoprotein by cholesterol-loaded macrophages. Arteriosclerosis and thrombosis : a journal of vascular biology 12, 745-753.

Olah, M., Amor, S., Brouwer, N., Vinet, J., Eggen, B., Biber, K., and Boddeke, H.W. (2012). Identification of a microglia phenotype supportive of remyelination. Glia 60, 306-321. Otasek, D., Morris, J.H., Boucas, J., Pico, A.R., and Demchak, B. (2019). Cytoscape Automation: empowering workflow-based network analysis. Genome biology 20, 185. Parkhurst, C.N., Yang, G., Ninan, I., Savas, J.N., Yates, J.R., 3rd, Lafaille, J.J., Hempstead, B.L., Littman, D.R., and Gan, W.B. (2013). Microglia promote learning-dependent synapse formation through brain-derived neurotrophic factor. Cell 155, 1596-1609.

Paterka, M., Voss, J.O., Werr, J., Reuter, E., Franck, S., Leuenberger, T., Herz, J., Radbruch, H., Bopp, T., Siffrin, V., et al. (2017). Dendritic cells tip the balance towards induction of regulatory $\mathrm{T}$ cells upon priming in experimental autoimmune encephalomyelitis. Journal of autoimmunity $76,108-114$.

Paul, D., Cowan, A.E., Ge, S., and Pachter, J.S. (2013). Novel 3D analysis of Claudin-5 reveals significant endothelial heterogeneity among CNS microvessels. Microvascular research 86, 1-10.

Pfrieger, F.W., and Ungerer, N. (2011). Cholesterol metabolism in neurons and astrocytes. Progress in lipid research 50, 357-371. 
Piedrahita, J.A., Zhang, S.H., Hagaman, J.R., Oliver, P.M., and Maeda, N. (1992).

Generation of mice carrying a mutant apolipoprotein $\mathrm{E}$ gene inactivated by gene targeting in embryonic stem cells. Proceedings of the National Academy of Sciences of the United States of America 89, 4471-4475.

Pierson, E., Simmons, S.B., Castelli, L., and Goverman, J.M. (2012). Mechanisms regulating regional localization of inflammation during CNS autoimmunity. Immunological reviews 248 , 205-215.

Pinholt, M., Frederiksen, J.L., and Christiansen, M. (2006). The association between apolipoprotein $E$ and multiple sclerosis. European journal of neurology 13, 573-580.

Pokharel, S.M., Shil, N.K., Gc, J.B., Colburn, Z.T., Tsai, S.Y., Segovia, J.A., Chang, T.H., Bandyopadhyay, S., Natesan, S., Jones, J.C.R., et al. (2019). Integrin activation by the lipid molecule 25-hydroxycholesterol induces a proinflammatory response. Nature communications 10, 1482.

Posokhova, E.N., Khoshchenko, O.M., Chasovskikh, M.I., Pivovarova, E.N., and Dushkin, M.I. (2008). Lipid synthesis in macrophages during inflammation in vivo: effect of agonists of peroxisome proliferator activated receptors alpha and gamma and of retinoid $\mathrm{X}$ receptors. Biochemistry. Biokhimiia 73, 296-304.

Quinet, E.M., Savio, D.A., Halpern, A.R., Chen, L., Miller, C.P., and Nambi, P. (2004). Geneselective modulation by a synthetic oxysterol ligand of the liver $X$ receptor. Journal of lipid research 45, 1929-1942.

Radzun, H.J., Hansmann, M.L., Heidebrecht, H.J., Bodewadt-Radzun, S., Wacker, H.H., Kreipe, H., Lumbeck, H., Hernandez, C., Kuhn, C., and Parwaresch, M.R. (1991). Detection of a monocyte/macrophage differentiation antigen in routinely processed paraffin-embedded tissues by monoclonal antibody Ki-M1P. Laboratory investigation; a journal of technical methods and pathology 65, 306-315.

Ransohoff, R.M. (2012). Animal models of multiple sclerosis: the good, the bad and the bottom line. Nature neuroscience 15, 1074-1077.

Relas, H., Gylling, H., and Miettinen, T.A. (2001). Fate of intravenously administered squalene and plant sterols in human subjects. Journal of lipid research 42, 988-994.

Rezai-Zadeh, K., Gate, D., and Town, T. (2009). CNS infiltration of peripheral immune cells: D-Day for neurodegenerative disease? Journal of neuroimmune pharmacology : the official journal of the Society on Neurolmmune Pharmacology 4, 462-475.

Rezapour-Firouzi, S., Arefhosseini, S.R., Mehdi, F., Mehrangiz, E.M., Baradaran, B.,

Sadeghihokmabad, E., Mostafaei, S., Fazljou, S.M., Torbati, M.A., Sanaie, S., et al. (2013). Immunomodulatory and therapeutic effects of Hot-nature diet and co-supplemented hemp seed, evening primrose oils intervention in multiple sclerosis patients. Complementary therapies in medicine 21, 473-480.

Ridder, D.A., Lang, M.F., Salinin, S., Roderer, J.P., Struss, M., Maser-Gluth, C., and Schwaninger, M. (2011). TAK1 in brain endothelial cells mediates fever and lethargy. The Journal of experimental medicine 208, 2615-2623.

Rodgers, J.M., Robinson, A.P., and Miller, S.D. (2013). Strategies for protecting oligodendrocytes and enhancing remyelination in multiple sclerosis. Discovery medicine 16 , 53-63.

Rossi-George, A., Guo, C.J., Oakes, B.L., and Gow, A.J. (2012). Copper modulates the phenotypic response of activated BV2 microglia through the release of nitric oxide. Nitric oxide : biology and chemistry $27,201-209$.

Rotsch, A.H., Kopka, J., Feussner, I., and Ischebeck, T. (2017). Central metabolite and sterol profiling divides tobacco male gametophyte development and pollen tube growth into eight metabolic phases. The Plant journal : for cell and molecular biology 92, 129-146.

Saeed, A.A., Genove, G., Li, T., Lutjohann, D., Olin, M., Mast, N., Pikuleva, I.A., Crick, P., Wang, Y., Griffiths, W., et al. (2014). Effects of a disrupted blood-brain barrier on cholesterol homeostasis in the brain. The Journal of biological chemistry 289, 23712-23722.

Saher, G., Brugger, B., Lappe-Siefke, C., Mobius, W., Tozawa, R., Wehr, M.C., Wieland, F., Ishibashi, S., and Nave, K.A. (2005). High cholesterol level is essential for myelin membrane growth. Nature neuroscience $8,468-475$. 
Saher, G., Rudolphi, F., Corthals, K., Ruhwedel, T., Schmidt, K.F., Lowel, S., Dibaj, P., Barrette, B., Mobius, W., and Nave, K.A. (2012). Therapy of Pelizaeus-Merzbacher disease in mice by feeding a cholesterol-enriched diet. Nature medicine 18, 1130-1135.

Saher, G., and Stumpf, S.K. (2015). Cholesterol in myelin biogenesis and hypomyelinating disorders. Biochimica et biophysica acta 1851, 1083-1094.

Schindelin, J., Arganda-Carreras, I., Frise, E., Kaynig, V., Longair, M., Pietzsch, T., Preibisch, S., Rueden, C., Saalfeld, S., Schmid, B., et al. (2012). Fiji: an open-source platform for biological-image analysis. Nature methods 9, 676-682.

Schuster, G.U., Parini, P., Wang, L., Alberti, S., Steffensen, K.R., Hansson, G.K., Angelin, B., and Gustafsson, J.A. (2002). Accumulation of foam cells in liver $X$ receptor-deficient mice.

Circulation 106, 1147-1153.

Secor McVoy, J.R., Oughli, H.A., and Oh, U. (2015). Liver X receptor-dependent inhibition of microglial nitric oxide synthase 2. Journal of neuroinflammation 12, 27.

Sedaghat, F., Jessri, M., Behrooz, M., Mirghotbi, M., and Rashidkhani, B. (2016).

Mediterranean diet adherence and risk of multiple sclerosis: a case-control study. Asia

Pacific journal of clinical nutrition 25, 377-384.

Shannon, P., Markiel, A., Ozier, O., Baliga, N.S., Wang, J.T., Ramage, D., Amin, N.,

Schwikowski, B., and Ideker, T. (2003). Cytoscape: a software environment for integrated models of biomolecular interaction networks. Genome research 13, 2498-2504.

Sharpe, L.J., and Brown, A.J. (2013). Controlling cholesterol synthesis beyond 3-hydroxy-3methylglutaryl-CoA reductase (HMGCR). The Journal of biological chemistry $288,18707-$ 18715.

Shibata, N., and Glass, C.K. (2009). Regulation of macrophage function in inflammation and atherosclerosis. Journal of lipid research 50 Suppl, S277-281.

Smets, I., Goris, A., Moisse, M., Vandebergh, M., Lagou, V., Demeestere, J., Mallants, K., Hilven, K., Van Damme, P., Sunaert, S., et al. (2018). Chr8p23 region implicated in genetic basis for demyelination in multiple sclerosis patients. In 34th Congress of the European-

Committee-for-Treatment-and-Research-in-Multiple-Sclerosis (ECTRIMS) (Berlin: ECTRIMS Online Library).

Smith, T.J. (2000). Squalene: potential chemopreventive agent. Expert opinion on investigational drugs 9, 1841-1848.

Solt, L.A., Kamenecka, T.M., and Burris, T.P. (2012). LXR-mediated inhibition of CD4+ T helper cells. PloS one 7, e46615.

Spann, N.J., Garmire, L.X., McDonald, J.G., Myers, D.S., Milne, S.B., Shibata, N., Reichart, D., Fox, J.N., Shaked, I., Heudobler, D., et al. (2012). Regulated accumulation of desmosterol integrates macrophage lipid metabolism and inflammatory responses. Cell 151, 138-152.

Stadelmann, C., Albert, M., Wegner, C., and Bruck, W. (2008). Cortical pathology in multiple sclerosis. Current opinion in neurology 21, 229-234.

Stafford, S.L., Bokil, N.J., Achard, M.E., Kapetanovic, R., Schembri, M.A., McEwan, A.G., and Sweet, M.J. (2013). Metal ions in macrophage antimicrobial pathways: emerging roles for zinc and copper. Bioscience reports 33.

Stuart, T., Butler, A., Hoffman, P., Hafemeister, C., Papalexi, E., Mauck, W.M., 3rd, Hao, Y., Stoeckius, M., Smibert, P., and Satija, R. (2019). Comprehensive Integration of Single-Cell Data. Cell 177, 1888-1902 e1821.

Stumpf, S.K., Berghoff, S.A., Trevisiol, A., Spieth, L., Duking, T., Schneider, L.V., Schlaphoff, L., Dreha-Kulaczewski, S., Bley, A., Burfeind, D., et al. (2019). Ketogenic diet ameliorates axonal defects and promotes myelination in Pelizaeus-Merzbacher disease. Acta neuropathologica 138, 147-161.

Subramanian, A., Tamayo, P., Mootha, V.K., Mukherjee, S., Ebert, B.L., Gillette, M.A., Paulovich, A., Pomeroy, S.L., Golub, T.R., Lander, E.S., et al. (2005). Gene set enrichment analysis: a knowledge-based approach for interpreting genome-wide expression profiles. Proceedings of the National Academy of Sciences of the United States of America 102, 15545-15550.

Sutermaster, B.A., and Darling, E.M. (2019). Considerations for high-yield, high-throughput cell enrichment: fluorescence versus magnetic sorting. Scientific reports 9, 227. 
Suzuki, K. (1969). Giant hepatic mitochondria: production in mice fed with cuprizone. Science 163, 81-82.

Thomas, D.G., Doran, A.C., Fotakis, P., Westerterp, M., Antonson, P., Jiang, H., Jiang, X.C., Gustafsson, J.A., Tabas, I., and Tall, A.R. (2018). LXR Suppresses Inflammatory Gene Expression and Neutrophil Migration through cis-Repression and Cholesterol Efflux. Cell reports 25, 3774-3785 e3774.

Torocsik, D., Szanto, A., and Nagy, L. (2009). Oxysterol signaling links cholesterol metabolism and inflammation via the liver $\mathrm{X}$ receptor in macrophages. Molecular aspects of medicine 30, 134-152.

Voskuhl, R.R., Itoh, N., Tassoni, A., Matsukawa, M.A., Ren, E., Tse, V., Jang, E., Suen, T.T., and Itoh, Y. (2019). Gene expression in oligodendrocytes during remyelination reveals cholesterol homeostasis as a therapeutic target in multiple sclerosis. Proceedings of the National Academy of Sciences of the United States of America 116, 10130-10139.

Vuletic, S., Kennedy, H., Albers, J.J., Killestein, J., Vrenken, H., Lutjohann, D., and Teunissen, C.E. (2014). Cerebrospinal fluid apolipoprotein E and phospholipid transfer protein activity are reduced in multiple sclerosis; relationships with the brain MRI and CSF lipid variables. Multiple sclerosis and related disorders 3, 533-541.

Wang, X., and Roper, M.G. (2014). Measurement of DCF fluorescence as a measure of reactive oxygen species in murine islets of Langerhans. Analytical methods : advancing methods and applications 6, 3019-3024.

Wang, Y., Sun, P., Wang, Q., Trinkaus, K., Schmidt, R.E., Naismith, R.T., Cross, A.H., and Song, S.K. (2015). Differentiation and quantification of inflammation, demyelination and axon injury or loss in multiple sclerosis. Brain : a journal of neurology 138, 1223-1238.

Wang, Z., Sadovnick, A.D., Traboulsee, A.L., Ross, J.P., Bernales, C.Q., Encarnacion, M., Yee, I.M., de Lemos, M., Greenwood, T., Lee, J.D., et al. (2016). Nuclear Receptor NR1H3 in Familial Multiple Sclerosis. Neuron 92, 555.

Westerterp, M., Gourion-Arsiquaud, S., Murphy, A.J., Shih, A., Cremers, S., Levine, R.L., Tall, A.R., and Yvan-Charvet, L. (2012). Regulation of hematopoietic stem and progenitor cell mobilization by cholesterol efflux pathways. Cell stem cell 11, 195-206.

Wickham, H. (2009). ggplot2 - Elegant Graphics for Data Analysis. (Springer).

Wilson, R., and Tocher, D.R. (1991). Lipid and fatty acid composition is altered in plaque tissue from multiple sclerosis brain compared with normal brain white matter. Lipids 26, 9-15. Winchenbach, J., Duking, T., Berghoff, S.A., Stumpf, S.K., Hulsmann, S., Nave, K.A., and Saher, G. (2016). Inducible targeting of CNS astrocytes in Aldh1l1-CreERT2 BAC transgenic mice. F1000Research 5, 2934.

Yang, C., McDonald, J.G., Patel, A., Zhang, Y., Umetani, M., Xu, F., Westover, E.J., Covey, D.F., Mangelsdorf, D.J., Cohen, J.C., et al. (2006). Sterol intermediates from cholesterol biosynthetic pathway as liver $X$ receptor ligands. The Journal of biological chemistry 281 , 27816-27826.

Yona, S., Kim, K.W., Wolf, Y., Mildner, A., Varol, D., Breker, M., Strauss-Ayali, D., Viukov, S., Guilliams, M., Misharin, A., et al. (2013). Fate mapping reveals origins and dynamics of monocytes and tissue macrophages under homeostasis. Immunity 38, 79-91.

Zhang, Y., Chen, K., Sloan, S.A., Bennett, M.L., Scholze, A.R., O'Keeffe, S., Phatnani, H.P., Guarnieri, P., Caneda, C., Ruderisch, N., et al. (2014). An RNA-sequencing transcriptome and splicing database of glia, neurons, and vascular cells of the cerebral cortex. The Journal of neuroscience : the official journal of the Society for Neuroscience 34, 11929-11947.

Zhao, S., Hu, X., Park, J., Zhu, Y., Zhu, Q., Li, H., Luo, C., Han, R., Cooper, N., and Qiu, M. (2007). Selective expression of LDLR and VLDLR in myelinating oligodendrocytes.

Developmental dynamics : an official publication of the American Association of Anatomists 236, 2708-2712. 


\section{Supplemental Table 1: List of primer sequences}

All primers used for expression analysis were intron-spanning (5'-3'; forward - reverse).

\begin{tabular}{|c|c|}
\hline Hprt & TCCTCCTCAGACCGCTTTT - CCTGGTTCATCATCGCTAATC \\
\hline RplpO & GATGCCCAGGGAAGACAG - ACAATGAAGCATTTTGGATAATCA \\
\hline Rps13 & CGAAAGCACCTTGAGAGGAA -TTCCAATTAGGTGGGAGCAC \\
\hline $18 S$ & AAATCAGTTATGGTTCCTTTGGTC - GCTCTAGAATTACCACAGTTATCCAA \\
\hline Gapdh & CTACATGGTCTACATGTTCCAGTA - TGATGGCATGGACTGTGGTCAT \\
\hline Actb & TGACAGGATGCAGAAGGAGA - CGCTCAGGAGGAGCAATG \\
\hline Car2 & CAAGCACAACGGACCAGA - ATGAGCAGAGGCTGTAGG \\
\hline Olig2 & AGACCGAGCCAACACCAG - AAGCTCTCGAATGATCCTTCTTT \\
\hline Plp1 & TCAGTCTATTGCCTTCCCTAGC - AGCATTCCATGGGAGAACAC \\
\hline Cspg4 & TCATACAGAATGTTCCCAGCA - GCAGAGGAGGTCTTGGTGAA \\
\hline Pdgfra & CTCTTGGAGATAGACTCCGTAG - ACTTCTCTTCCTGCGAATGG \\
\hline Gfap & TGCTCCTGCTTCGAGTCCTT - CAAGAGGAACATCGTGGTAAAGA \\
\hline Aqp4 & TGGAGGATTGGGAGTCACC - TGAACACCAACTGGAAAGTGA \\
\hline S100b & AACAACGAGCTCTCTCACTTCC - CTCCATCACTTTGTCCACCA \\
\hline Aif1 & TGTTTTTCTCCTCATACATCAGAATC - CCGAGGAGACGTTCAGCTAC \\
\hline Lamp2 & AAGGTGCAACCTTTTAATGTGAC - TGTCATCATCCAGCGAACAC \\
\hline Hmgc1 & GGACCGCTGCTATTCTGTCT - AGCCAAAATCATTCAGGGTAAA \\
\hline $\mathrm{HmgCr}$ & TGATTGGAGTTGGCACCAT - TGGCCAACACTGACATGC \\
\hline Fdft1 & CCAAACAGGACTGGGACAAG - GACGAGAAAGGCCAATTCC \\
\hline Dhcr24 & GGTCATGACGGACGACGTA - AGGGCTTGTAGTAACTGCCAAT \\
\hline Cyp51 & TTAGAACAGAAAGCAGTGTGTGG - TGCATCTATCAAGTAAATTCAGATCC \\
\hline Mvk & CTCAAGGACGGGGTCTCC - GGCCCACTTGTTGATTGACT \\
\hline Apoe & GACCCTGGAGGCTAAGGACT - AGAGCCTTCATCTTCGCAAT \\
\hline Apod & ACGGAAACATCGAAGTGCTAA - TGGCTTCACCCTTTACTTGG \\
\hline Clu & GCCATGGATGTCCAGCTC - CACACAGTGCGGTCATCTTC \\
\hline Apoa1 & TCAGGATGAAAGCTGTGGTG - TCATCTTGCTGCCATACGTG \\
\hline Ldlr & GATGGCTATACCTACCCCTCAA - TGCTCATGCCACATCGTC \\
\hline VIdlr & AAGTCAGTGTTCCCCCAAAA - TGCTGCCATCACTAAGAGCA \\
\hline Apobr & TCACAGCAACTGGAATGAGG - GCTTCGAGAGAGGAGCACAC \\
\hline Lrp1 & ACCACCATCGTGGAAAATG - GTCCCAGCCACGGTGATA \\
\hline Scarb1 & GCCCATCATCTGCCAACT - TCCTGGGAGCCCTTTTTACT \\
\hline Pltp & GCTTCTGAGGGCCACCTAC - TTCAGCTTCAGTGGGGAGTT \\
\hline Abca1 & CTGTTTCCCCCAACTTCTG - TCTGCTCCATCTCTGCTTTC \\
\hline Abcg1 & TCTTTGATGAGCCCACCAGT - GGGCCAGTCCTTTCATCA \\
\hline Abcg 4 & TGATGTGCCCTTCCAGGT - CAAGGCTGAGAAGAGCAGGA \\
\hline Srebp2 & ACCTAGACCTCGCCAAAGGT - GCACGGATAAGCAGGTTTGT \\
\hline Npc1 & CCTTCGGGCCTCCATTG - TGTCACGGTTTCATTGTTGTAAGA \\
\hline Npc2 & CCGGTGAAGAATGAATACCC - TTCTTTTTGTCATCTTCAAGTTTCC \\
\hline Cyp46a1 & AACTTTGTCACCTTCTTCATTGC - CCATCACTGTGAATGCCAGA \\
\hline Ch25h & TGCTACAACGGTTCGGAGC - AGAAGCCCACGTAAGTGATGAT \\
\hline Cyp27a1 & ATGGGATCTTCATCGCACA - CGTTTAAGGCATCCGTGTAGA \\
\hline Tnf & TGCCTATGTCTCAGCCTCTTC - GAGGCCATTTGGGAACTTCT \\
\hline $1 / 1 b$ & GCTACCAAACTGGATATAATCAGGA - CCAGGTAGCTATGGTACTCCAGAA \\
\hline 112 & CGCAGAGGTCCAAGTTCATCT - CGCAGAGGTCCAAGTTCATCT \\
\hline 116 & GCTACCAAACTGGATATAATCAGGA - CCAGGTAGCTATGGTACTCCAGAA \\
\hline Ccl2 & GCCTGCTGTTCACAGTTGC - CAGGTGAGTGGGGCGTTA \\
\hline Cxcl10 & GCTGCCGTCATTTTCTGC - TCTCACTGGCCCGTCATC \\
\hline Ifng & TCAAGTGGCATAGATGTGGAAGAA - TGGCTCTGCAGGATTTTCATG \\
\hline H2-DMb2 (Mhcll) & GCGACGTGGGCGAGTACC - CATTCCGGAACCAGCGCA \\
\hline Nos2 & TGAACTTGAGCGAGGAGCA - TTCATGATAACGTTTCTGGCTCT \\
\hline Csf2 & GGGCGCCTTGAACATGAC - CGCATAGGTGGTAACTTGTGTTTC \\
\hline Fcgr3a & TCACCATCACTGTCCAAGACC - ACTAGGGAGAAAGCAGTGTGGT \\
\hline $1 / 10$ & GGTTGCCAAGCCTTATCGGA - ACCTGCTCCACTGCCTTGCT \\
\hline /112 & CCAGGTGTCTTAGCCAGTCC - GCAGTGCAGGAATAATGTTTCA \\
\hline Tgfb1 & TGGAGCAACATGTGGAACTC - CAGCAGCCGGTTACCAAG \\
\hline Mrc1 & AAGGAAAGTTCCCAGATGTACC - GCAAGCCAATGTACACGATG \\
\hline $\operatorname{Arg} 1$ & AAGGAAAGTTCCCAGATGTACC - GCAAGCCAATGTACACGATG \\
\hline NIrp3 & TGCGTGTTCTCTGTATACCAC - GGCTTAGGTCCACACAGAAAG \\
\hline Casp1 & CCCACTGCTGATAGGGTGAC - GCATAGGTACATAAGAATGAACTGGA \\
\hline
\end{tabular}


Supplemental Table 2: Patient characteristics

\begin{tabular}{cccccc} 
& Diagnosis & Sex & Age & $\begin{array}{c}\text { ApoE } \\
\text { genotype }\end{array}$ & $\begin{array}{c}\text { Post mortem } \\
\text { delay }\end{array}$ \\
\hline Patient 1 & Multiple sclerosis & $\mathrm{m}$ & 47 & 33 & $7: 15$ \\
\hline Patient 2 & Multiple sclerosis & $\mathrm{m}$ & 61 & N.A. & $9: 15$ \\
\hline Patient 3 & Multiple sclerosis & $\mathrm{f}$ & 47 & N.A. & $8: 35$ \\
\hline Patient 4 & Multiple sclerosis & $\mathrm{m}$ & 51 & 33 & $11: 00$ \\
\hline Control 1 & $\begin{array}{c}\text { Non-demented } \\
\text { control }\end{array}$ & $\mathrm{m}$ & 49 & N.A. & $6: 15$ \\
\hline Control 2 & $\begin{array}{c}\text { Non-demented } \\
\text { control }\end{array}$ & $\mathrm{f}$ & 46 & 33 & $10: 25$ \\
\hline Control 3 & $\begin{array}{c}\text { Control with space- } \\
\text { occupying process }\end{array}$ & $\mathrm{m}$ & 58 & N.A. & $05: 15$
\end{tabular}

Supplemental Table 3: Mean expression level of inflammatory cell clusters

\begin{tabular}{c|c|c|c|c|c|c|}
\multirow{2}{*}{} & \multicolumn{2}{|c|}{ Cluster 1 } & \multicolumn{2}{c|}{ Cluster 2 } & \multicolumn{2}{c|}{ Cluster 3 } \\
\cline { 2 - 7 } & Ctrl & MS & Ctrl & MS & Ctrl & MS \\
\hline DHCR24 & 0.16 & 0.10 & 0.45 & 0.06 & 1.216 & 1.05 \\
\hline ABCA1 & 0.40 & 1.54 & 4.43 & 8.23 & 0.82 & 1.91 \\
\hline APOE & 8.63 & 13.17 & 68.78 & 78.51 & 7.43 & 8.23 \\
\hline NR1H2 & 0.51 & 0.82 & 1.22 & 0.74 & 0.64 & 0.77 \\
\hline NR1H3 & 0.035 & 0.04 & 0.21 & 0.64 & 0.04 & 0.24
\end{tabular}


Figure 1

A

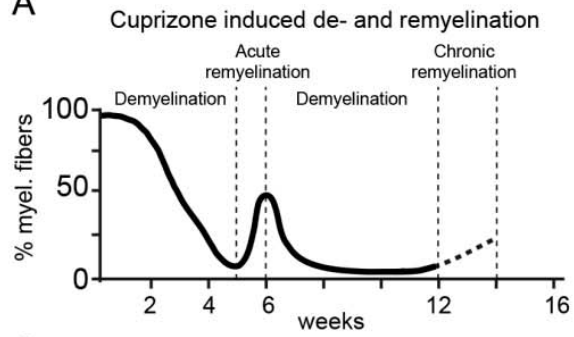

C

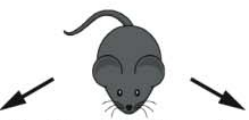

Acute remyelination Chronic remyelination

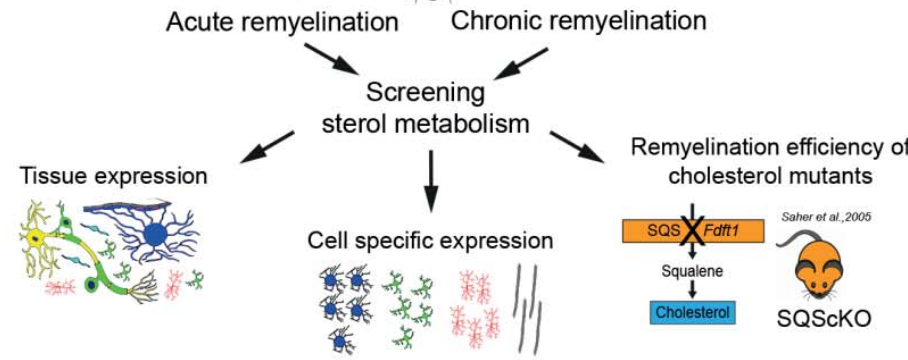

D MACS based isolation
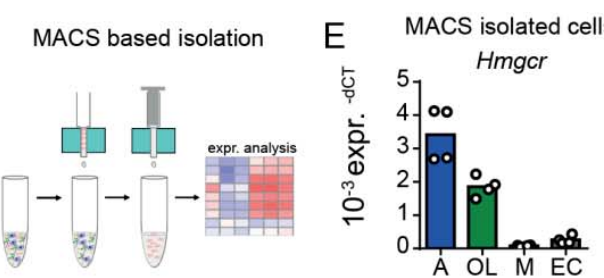

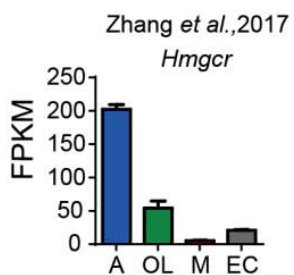

B
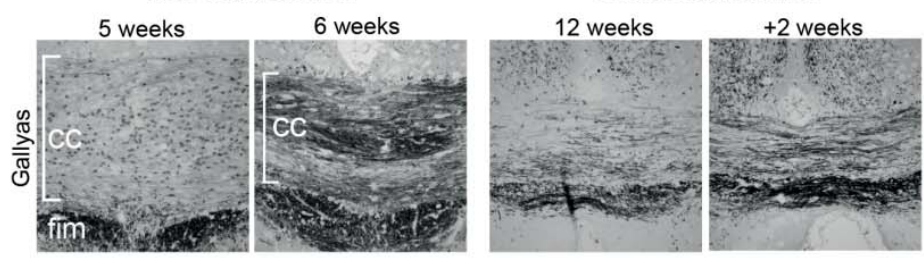

$\mathrm{F}$

Targeted sterol gene expression profile in tissue during actue and chronic remyelination

\begin{tabular}{c|l|l|}
\multicolumn{3}{c}{ acute } \\
\multicolumn{3}{|c}{$5 w$} \\
Hmgcr & & \\
Fdf1 & & \\
Cyp51 & & \\
Dhcr24 & & \\
\hline Ldlr & & \\
VIdlr & & \\
Apobr & & \\
Scarbl & & \\
Lrp1 & & \\
Tspo & & \\
Pltp & & \\
\hline Apoe & & \\
Clu & & \\
Apoa1 & & \\
Lcat & & \\
\hline Abca1 & & \\
Abcg1 & & \\
Abcg4 & & \\
\hline Soat1 & & \\
Nceh1 & & \\
Npc1 & & \\
Npc2 & & \\
\hline Cyp46a1 & & \\
Cyp27a1 & & \\
Ch25h & & \\
\hline
\end{tabular}
chronic

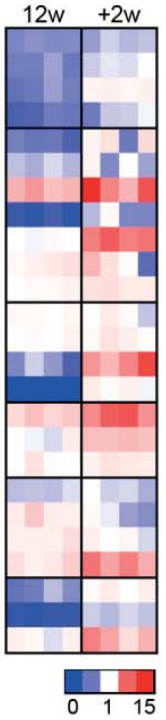

G

Targeted sterol gene expression profile of isolated cells during acute and chronic remyelination

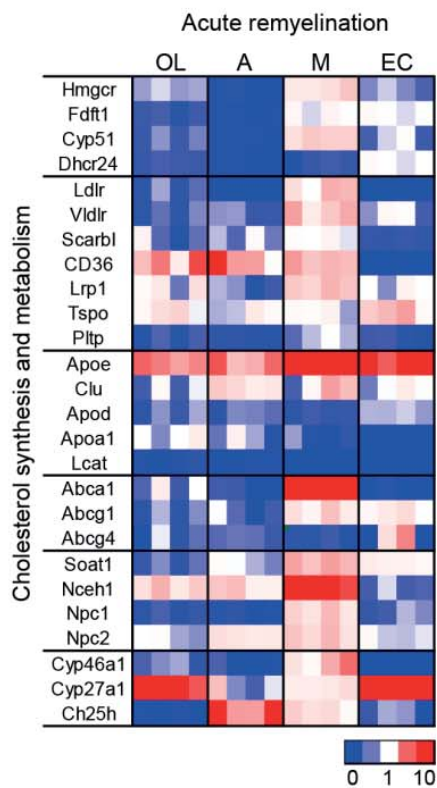

Chronic remyelination

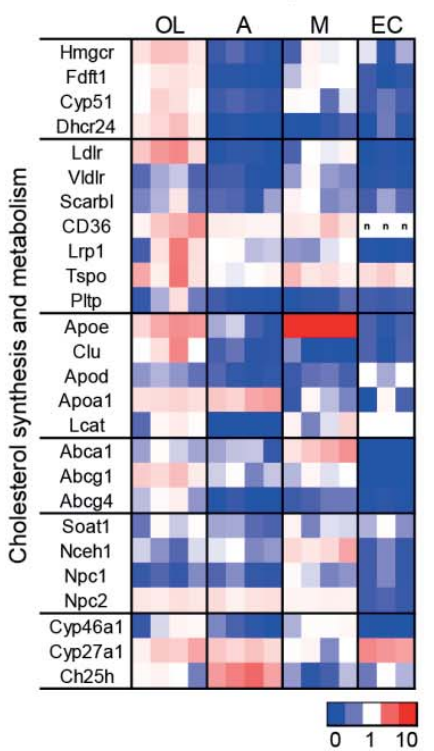

$\mathrm{H}$

Remyelination efficiency of cell specific cholesterol synthesis mutants

SQScKO

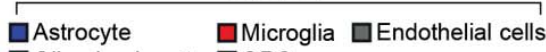
$\square$ Oligodendrocyte $\square$ OPC

$$
\text { Acute }
$$

remyelination

Chronic
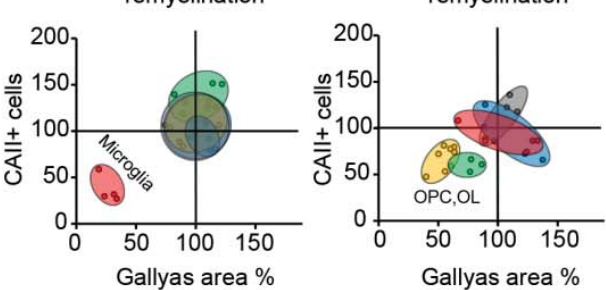

Gliosis
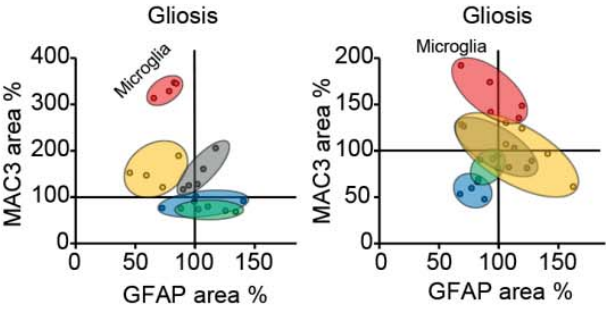
Figure 2

A Microglial cholesterol synthesis and export deficiency

B Microglial SQS and ABCA1/G1 ablation impacts on
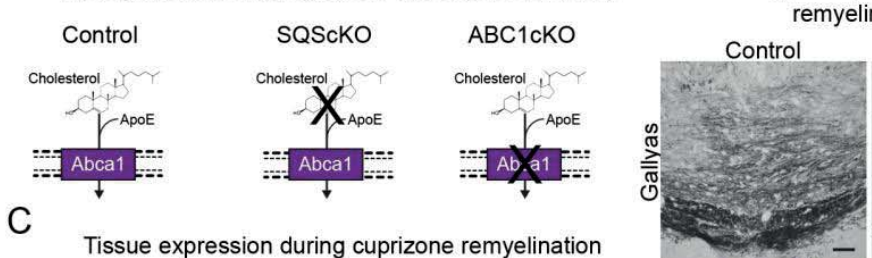

remyelination in the cuprizone model
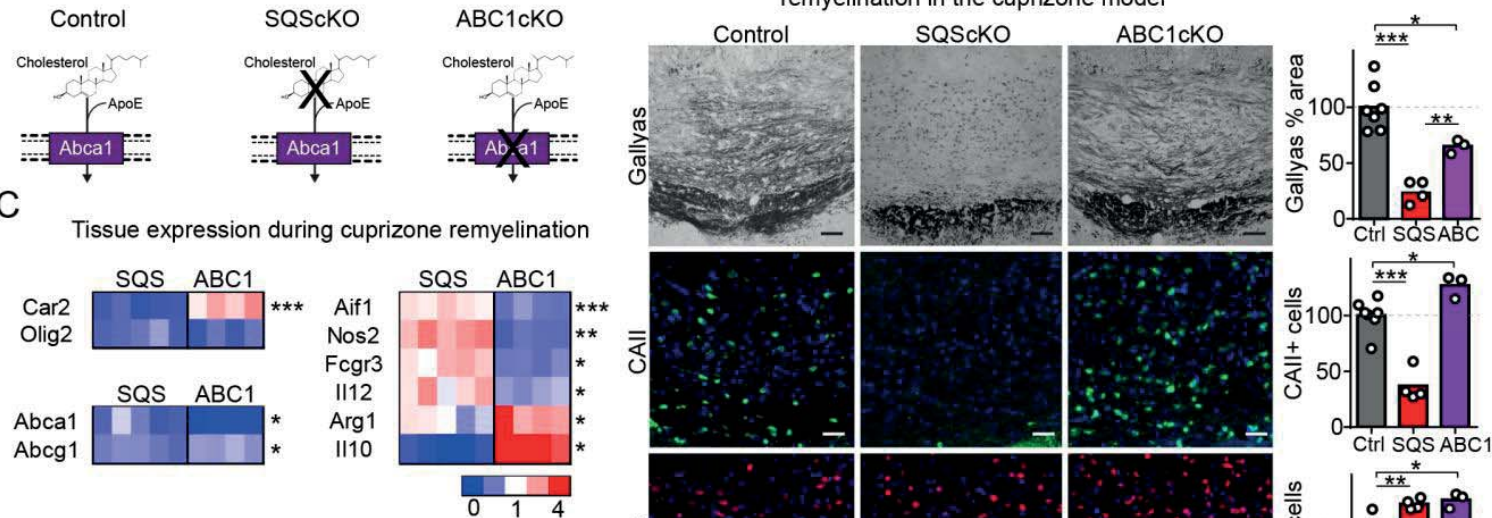

D

SQScKO in vitro phagocytosis
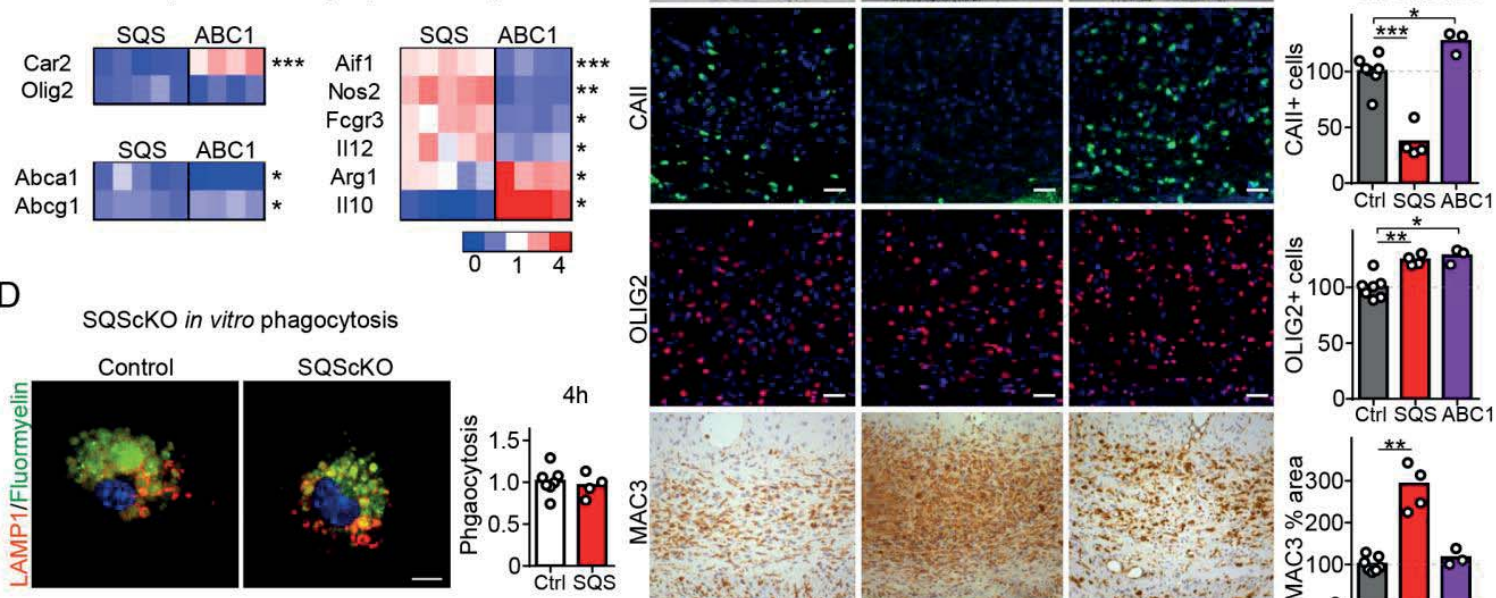

Foamy phagocytes and cholesterol crystals

G
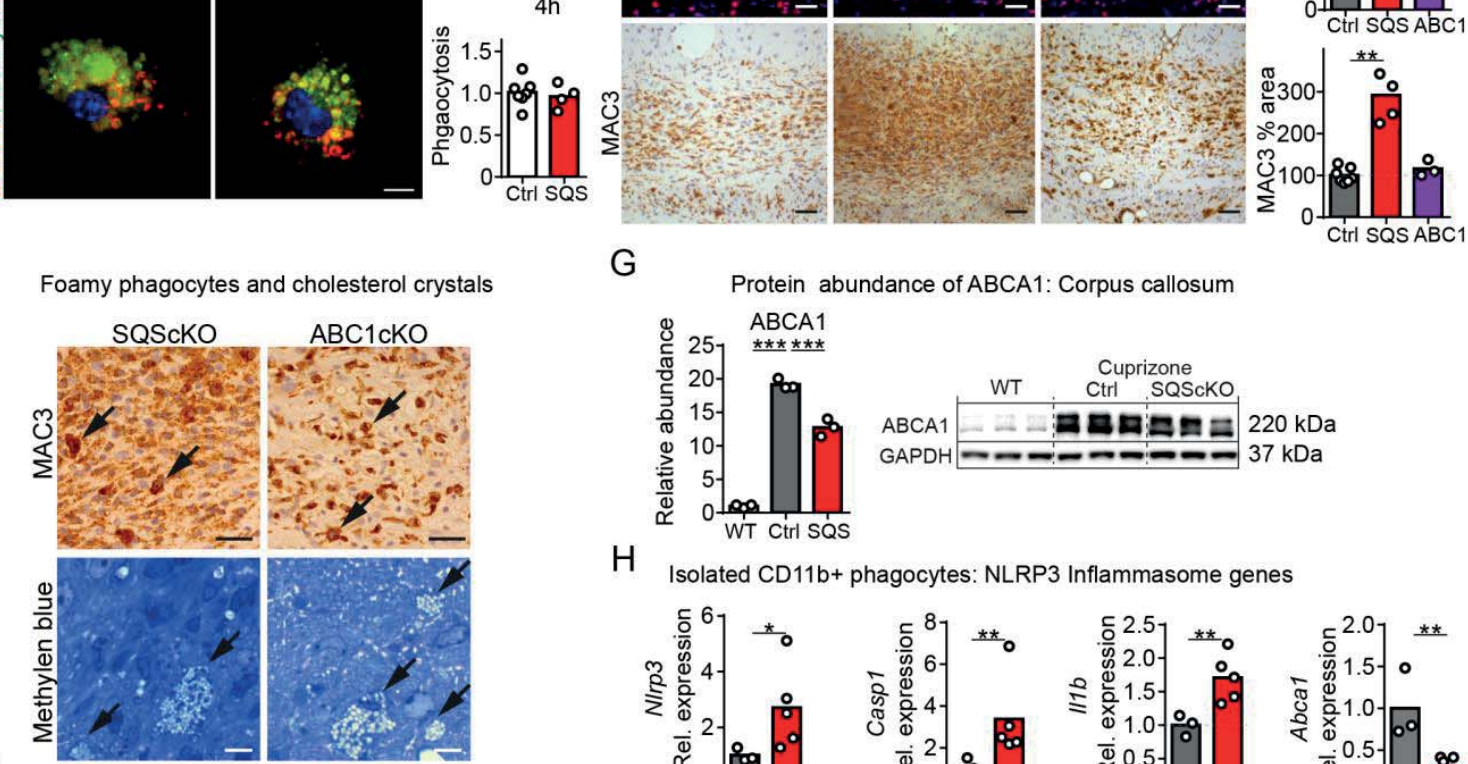

F

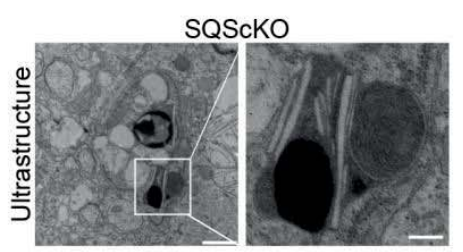

Protein abundance of $A B C A 1$ : Corpus callosum

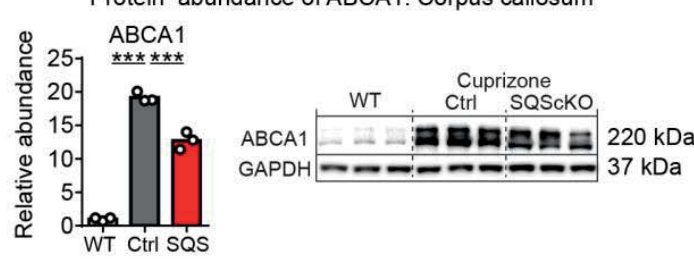

$\mathrm{H}$

Isolated CD11b+ phagocytes: NLRP3 Inflammasome genes

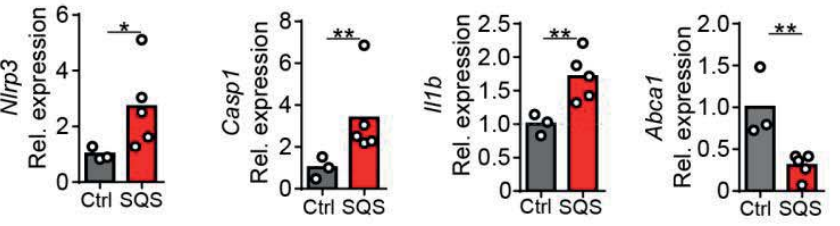

in vitro phagocytosis

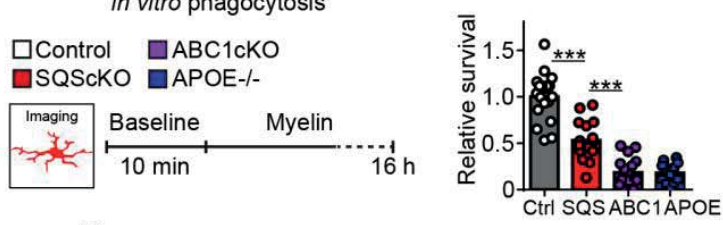

J SQScKO effects on microglial reactivity in lysolecithin lesions

$$
\text { K SQS deficiency effects on EAE disease severity }
$$
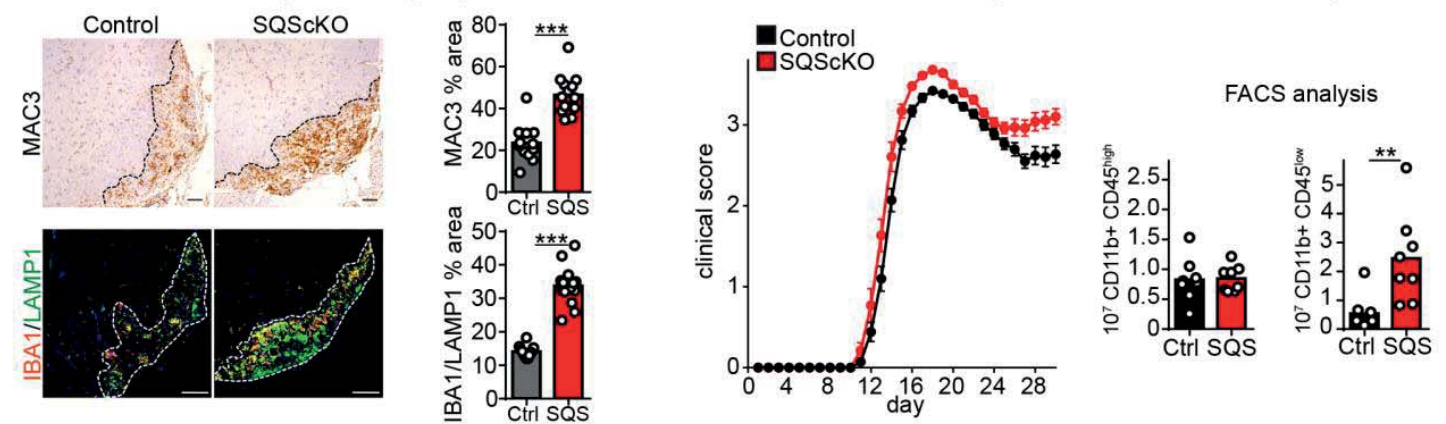
Figure 3

A
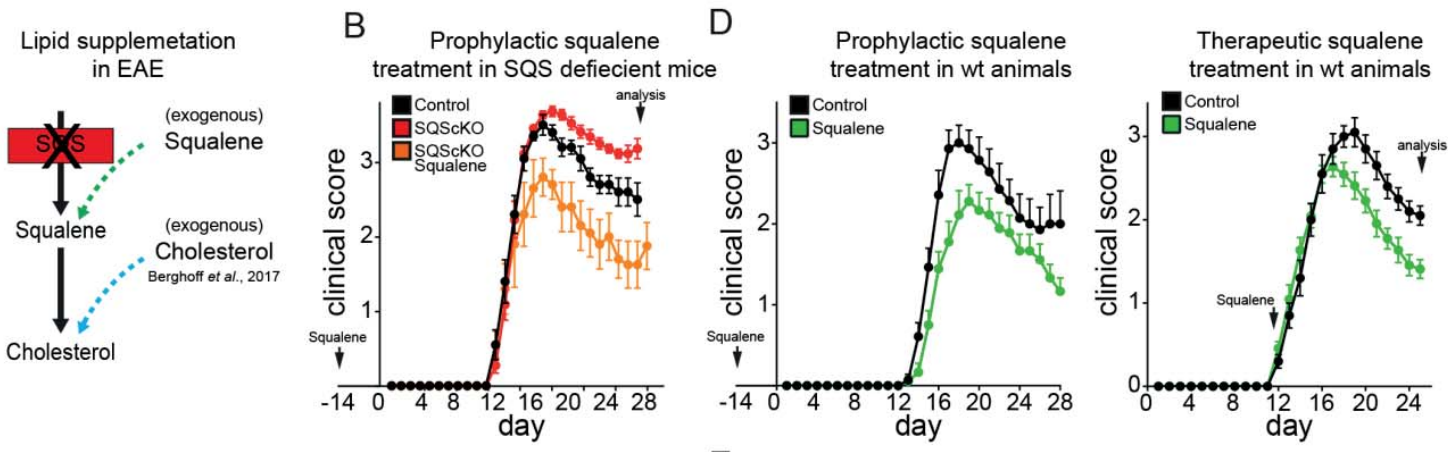

C

FACS analysis: Squalene treatment in SQScKO animals

E

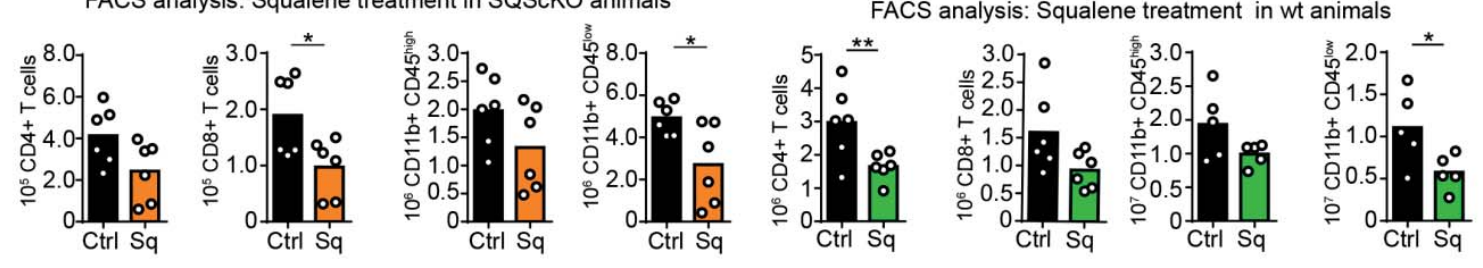

F

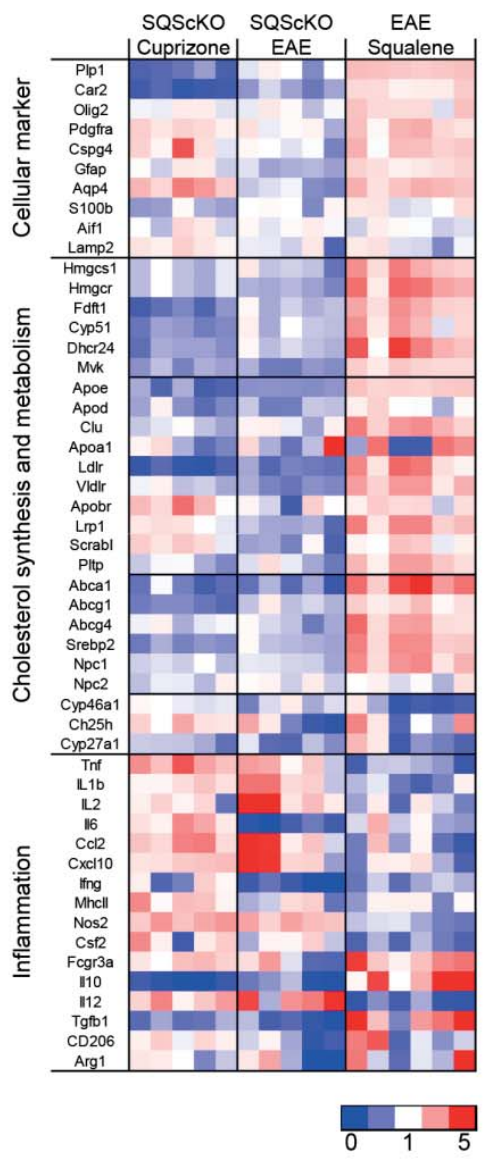

G

Targeted expression pofile of isolated CD11b+ cells

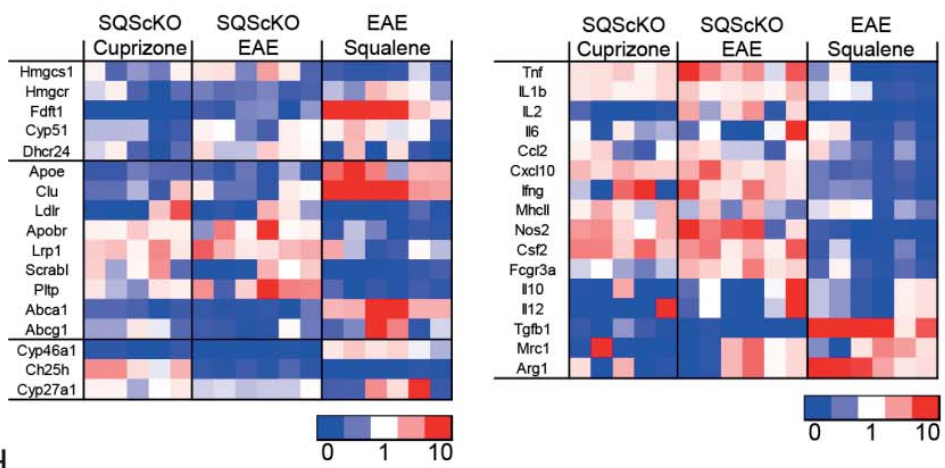

enn diagram of significantly
regulated genes

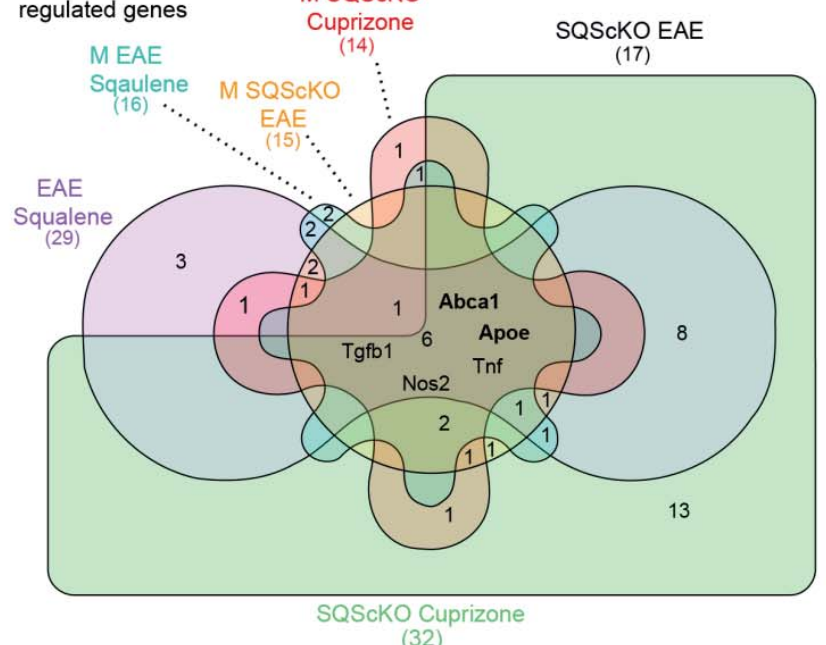


Figure 4

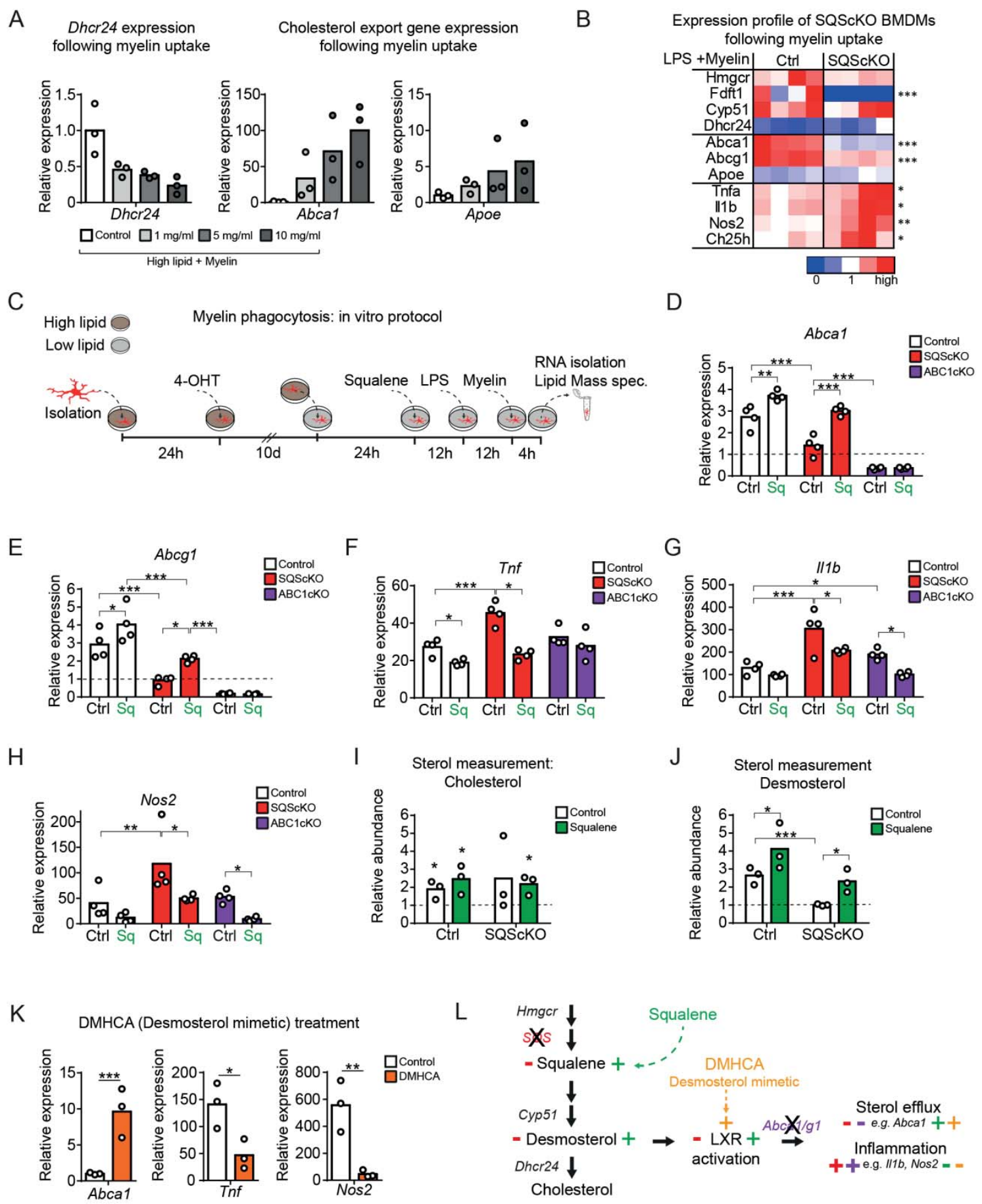


Figure 5

A Sterol synthesis gene expression in acutely isolated CD11b+myeloid cells
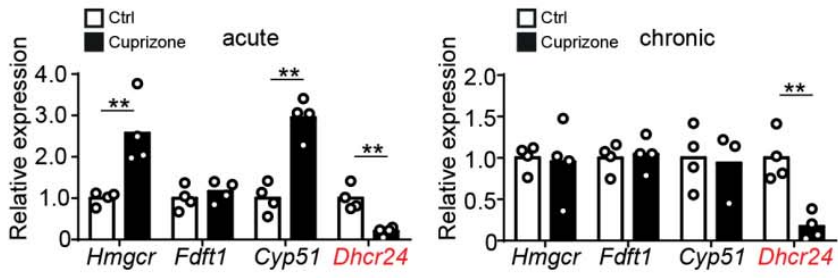

B
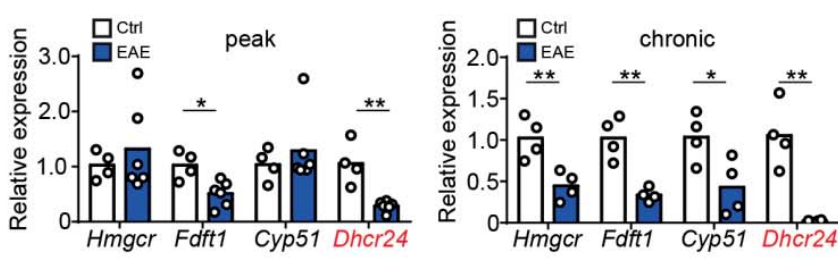

E

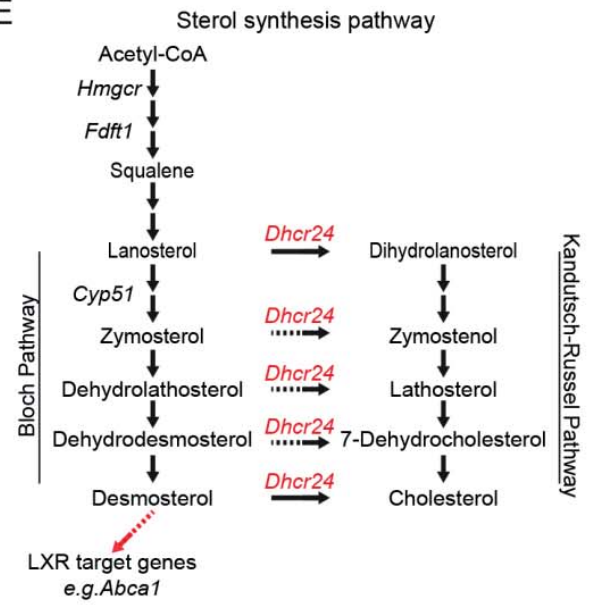

C

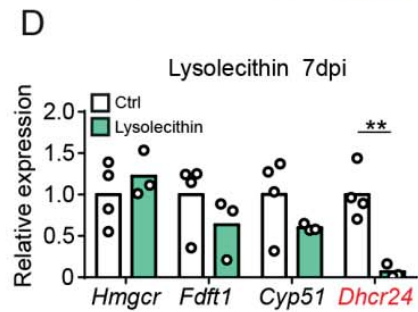

$\mathrm{F}$

G CD11b+cells:
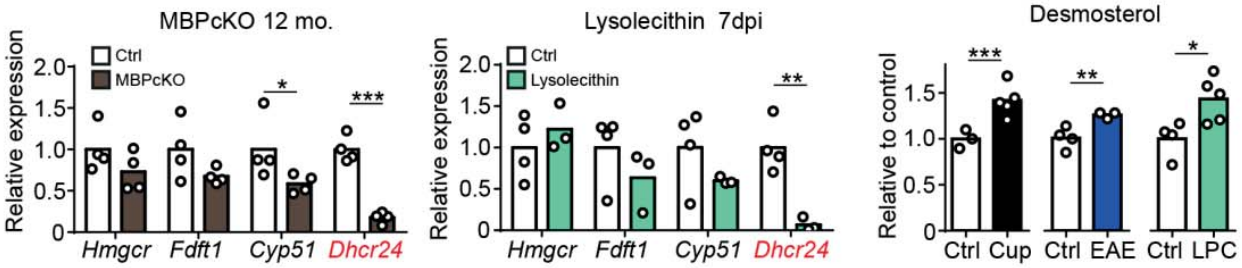

$\mathrm{H}$
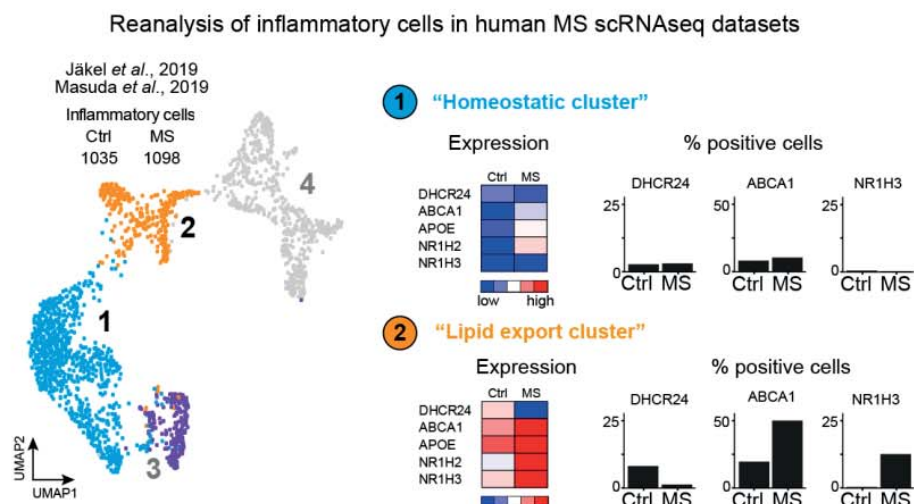

(2) "Lipid export cluster"
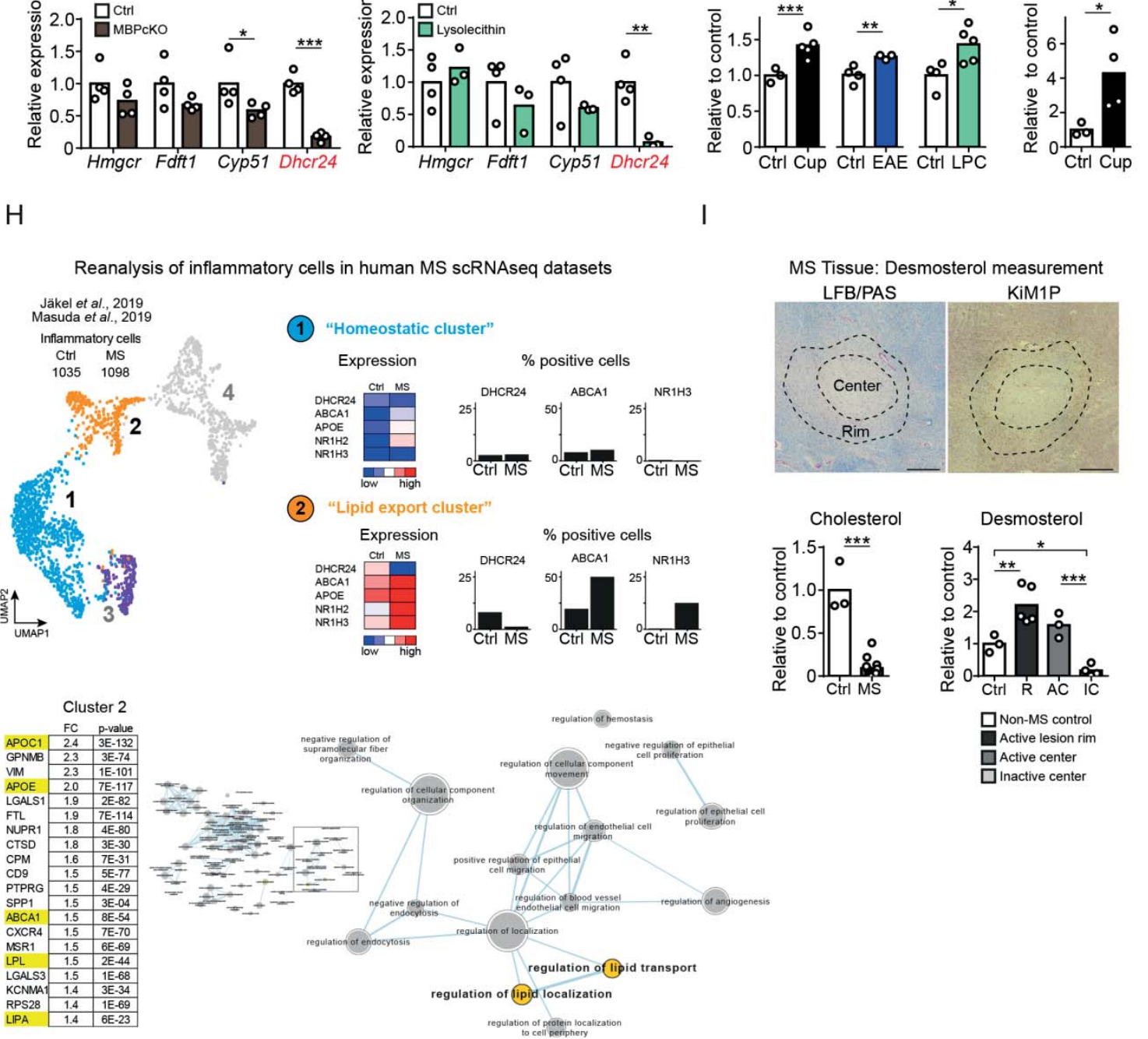

Non-MS control

Active lesion rim

$\square$ Active center 
Figure 6

A Squalene effects on oligodendrocyte myelination in vitro

B Squalene effects on oligodendrocyte differentiation in lysolecithin lesions

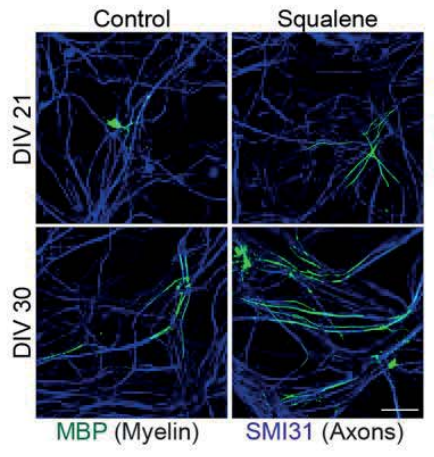

C

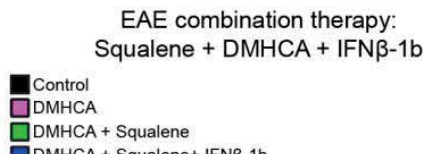

DMHCA + Squalene+ IFNB-1

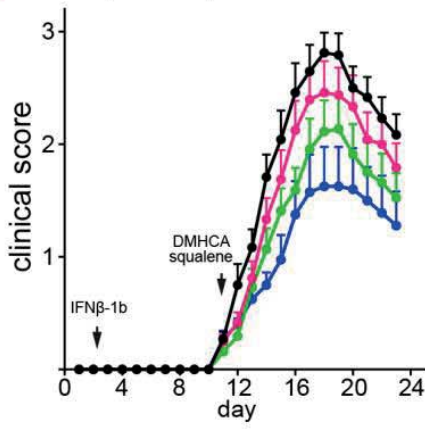

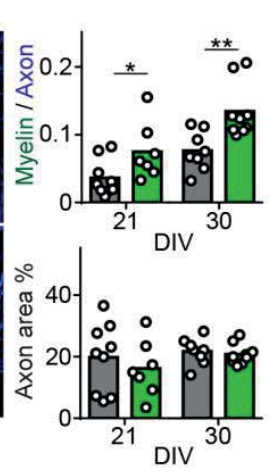

D

FACS analysis: Squalene+ DMHCA + IFN $\beta-1 b$
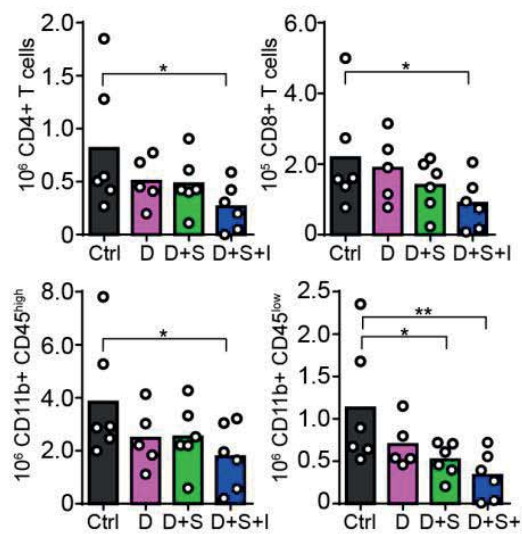

Squalene
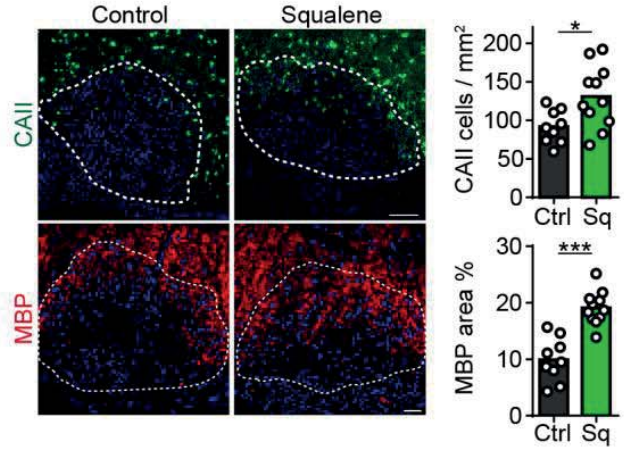

E

Therapeutic EAE in vivo sorted $C D 11 b+$ cells
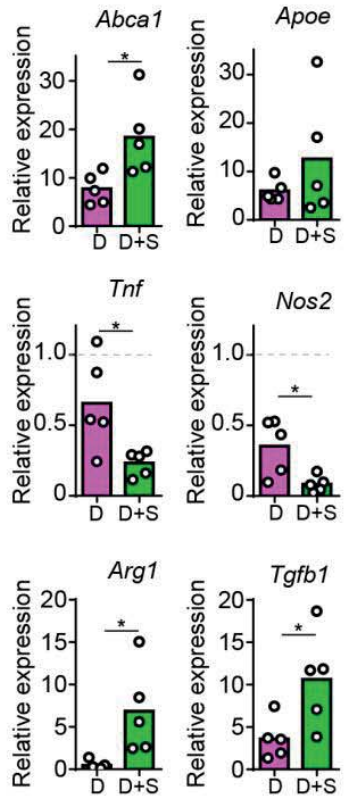


\section{Supplementary Figure 1}

A Cellular marker gene expression during remyelination

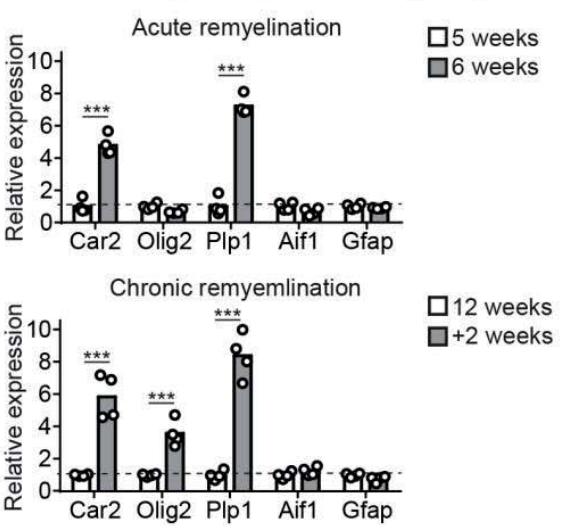

C

SQS KO efficiency: SQS expression of in vivo sorted cells

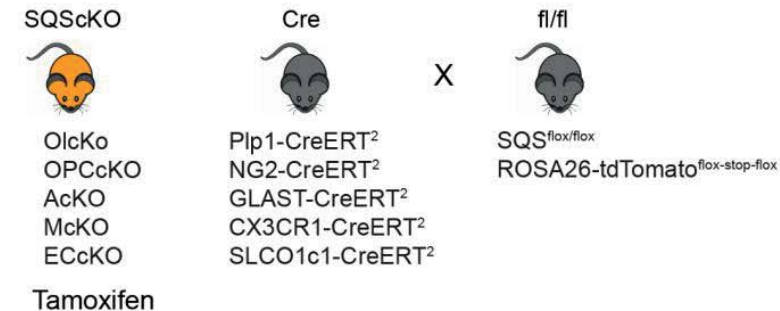

Tamoxifen

$\left|\begin{array}{l}1 \\ \mathrm{t}\end{array}\right|^{2}\left|\begin{array}{l}3 \\ \mathrm{t}\end{array}\right|^{4}\left|\begin{array}{l}5 \\ \mathrm{t}\end{array}\right|$

5 days

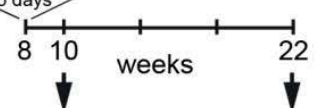

cell isolation evaluation

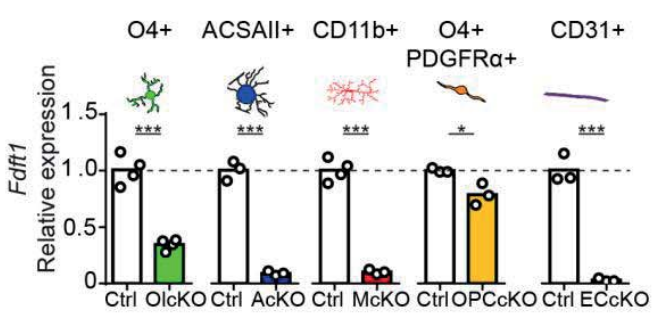

E

Cuprizone model: Tamoxifen protocols

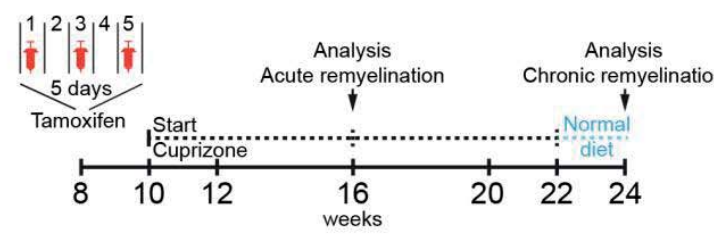

Mice: AcKO, McKO, OPCcKO, ECcKO

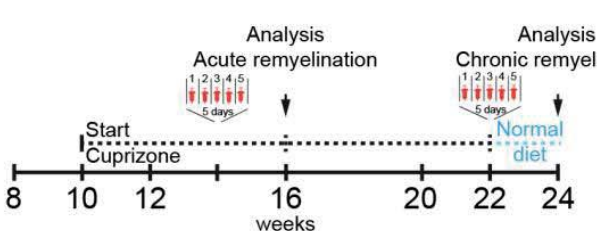

Mice: OLcKO
B Targeted sterol gene expression profil of isolated cells
from wildtype animals

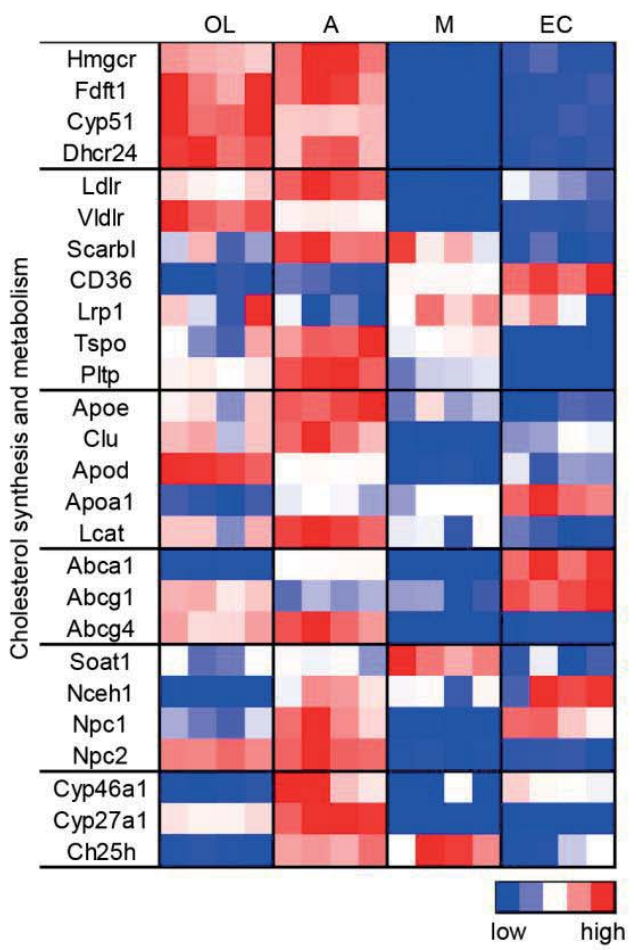

D

Peripheral monitoring of cholesterol mutants
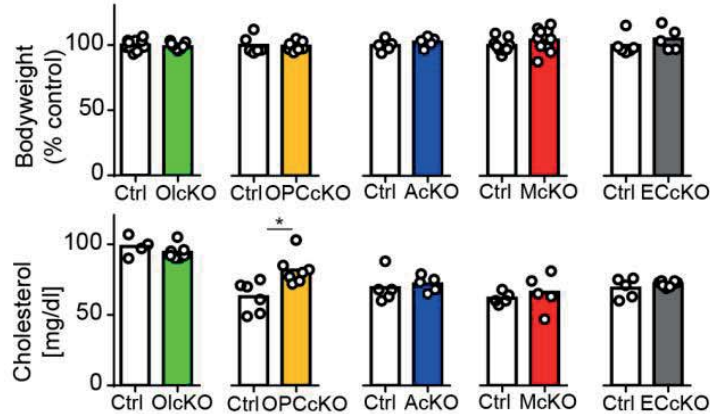

F Cell specific targeting during acute remyelination: Cre-reporter mice

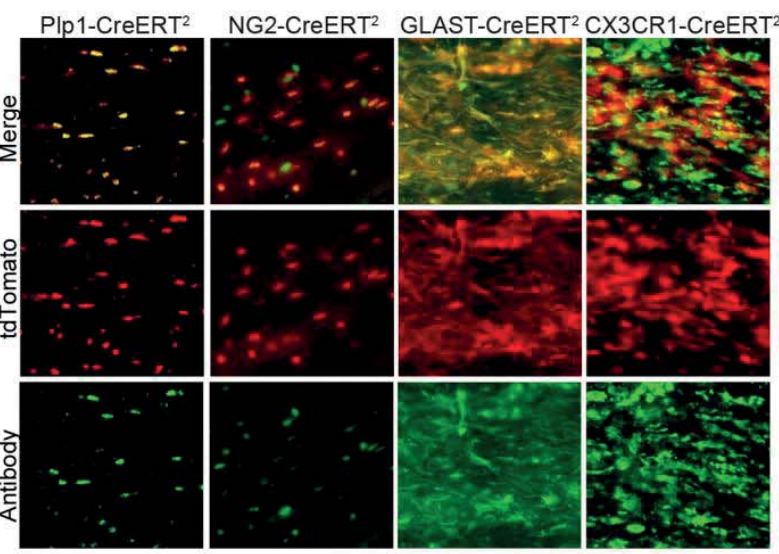

IBA1 


\section{Supplementary Figure 2}

A

Astrocytic expression of cholesterol synthesis genes in vivo

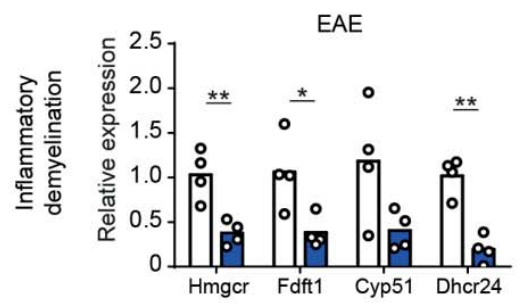

B
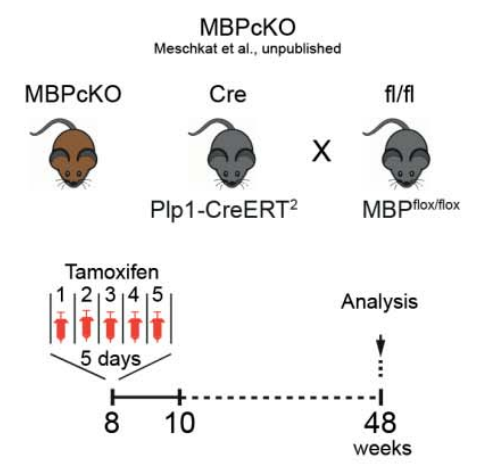

Astrocytic expression of cholesterol synthesis genes in vivo

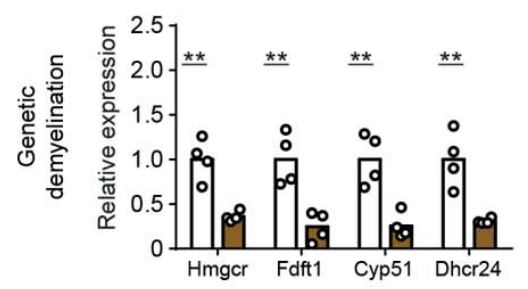

C

Aldh1/1-CreERT2 $\times$ SQS fi/f Winchenbach et al.,2016

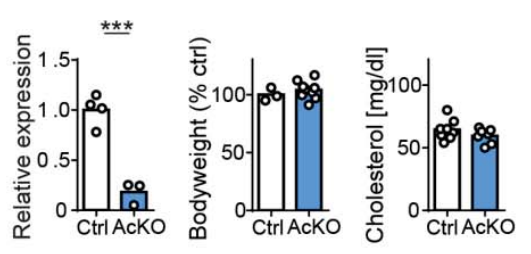

D

Remyelination efficiency of Aldh111-CreERT2 $\times$ SQS fiff

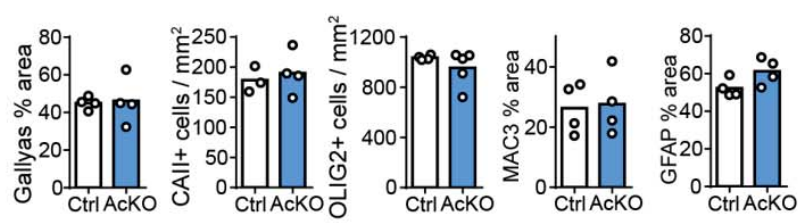

E

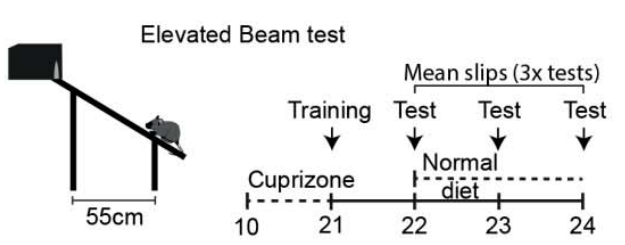

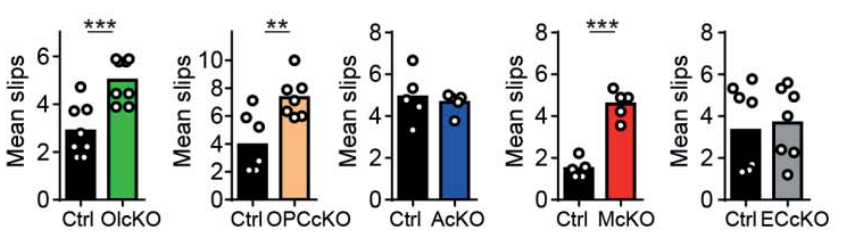




\section{Supplementary Figure 3}

A

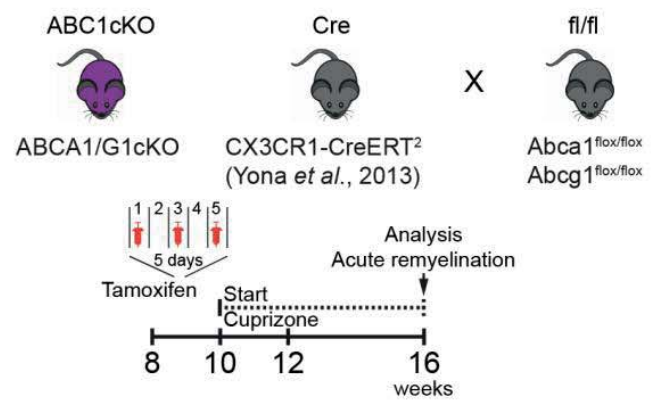

C

SQScKO BMDMs (in vitro): Lipid depleted media
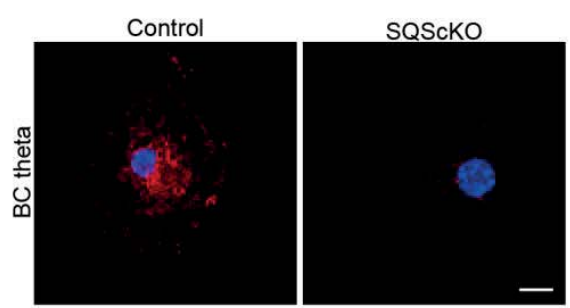

F
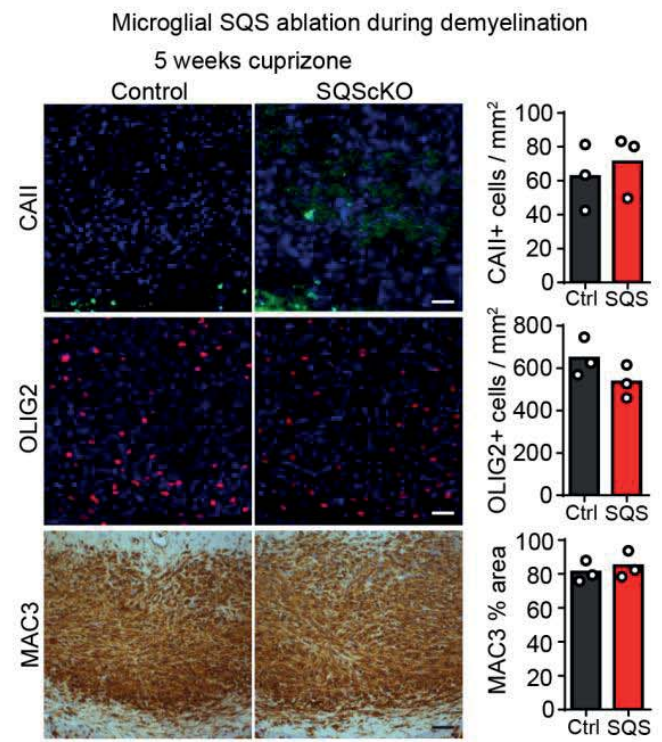

I

$$
\text { Lesion area }
$$

Lysolecithon SQScKO
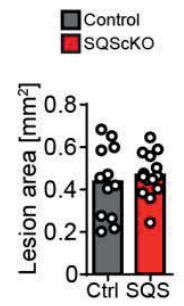

B

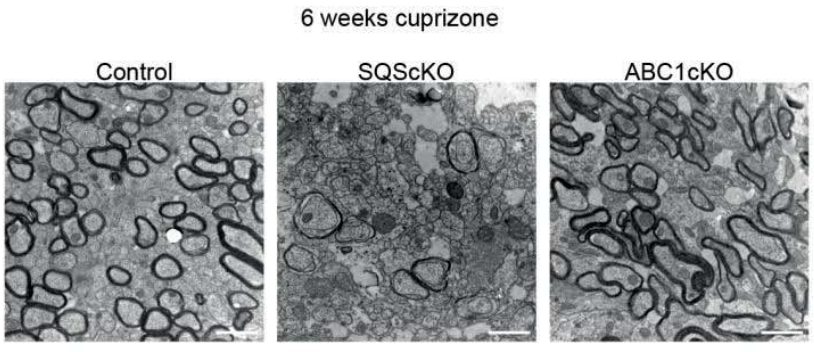

D SQS ablation in vitro

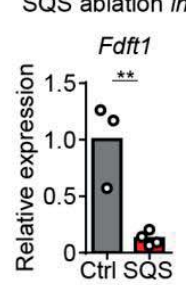

E in vitro phagocytosis

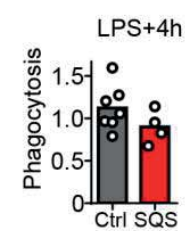

G

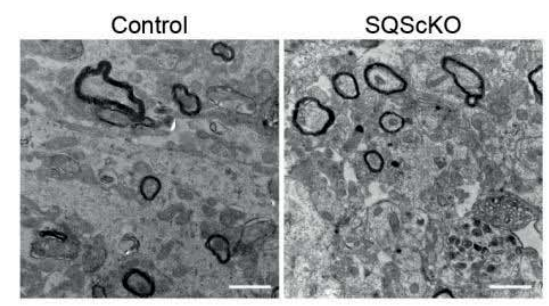

$\mathrm{H}$

Abca1/g1 ablation in vitro

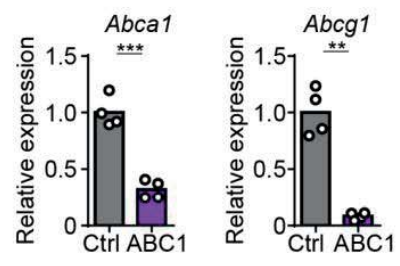

J

SQSCKO EAE tamoxifen protocol

K

FACS analysis EAE microglial SQScKO

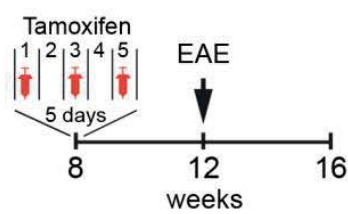

\section{Control}

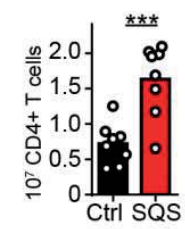


Supplementary Figure 4

A

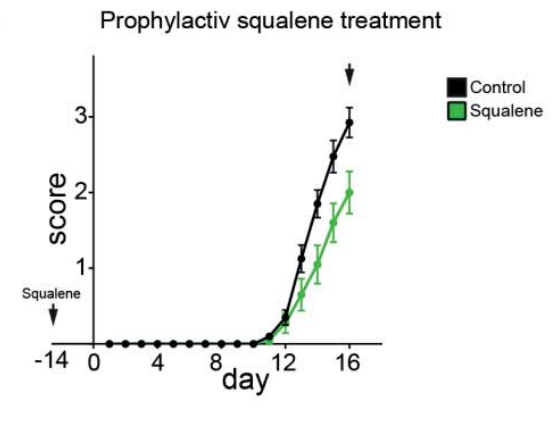

FACS analysis: Blood EAE Squalene vs. Control

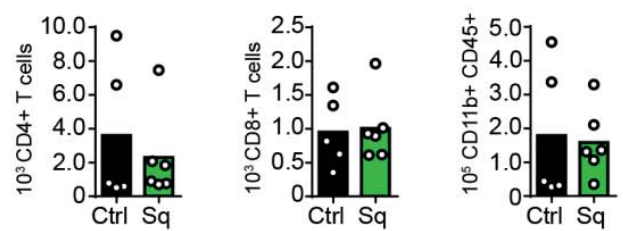

F

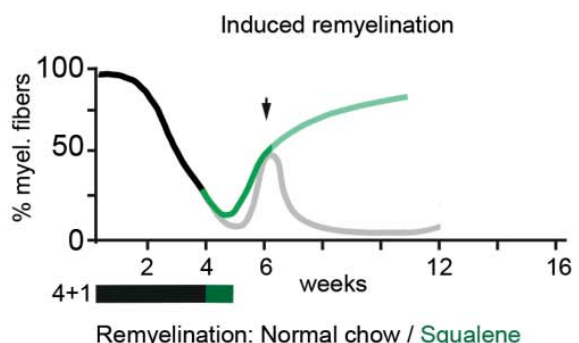

B

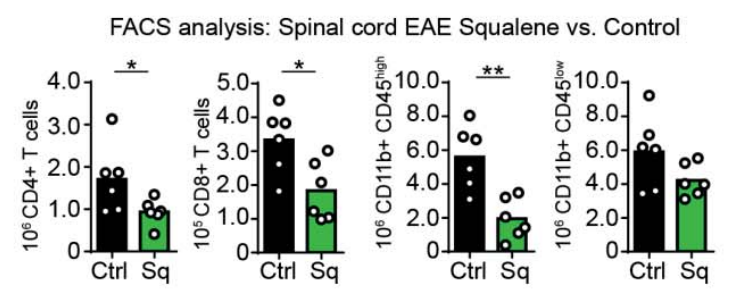

D

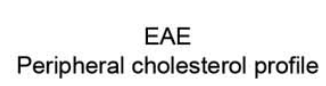

E

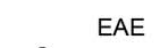

Serum squalene
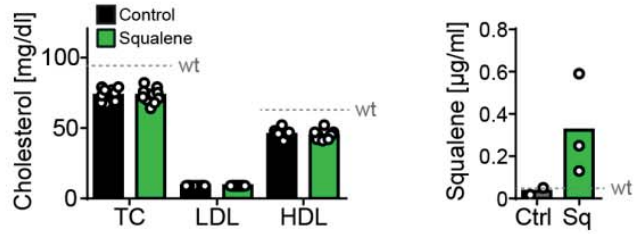

G

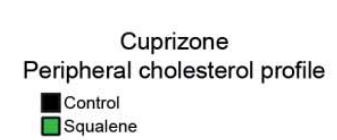

H Cuprizone Serum squalene
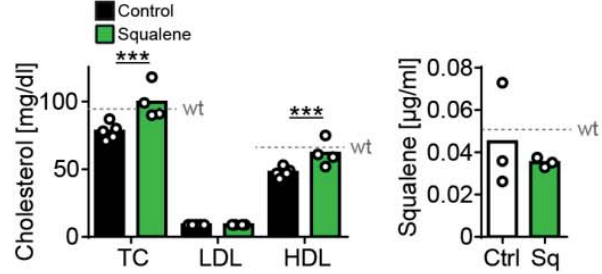

I

Cuprizone

Induced remyelination: Histology

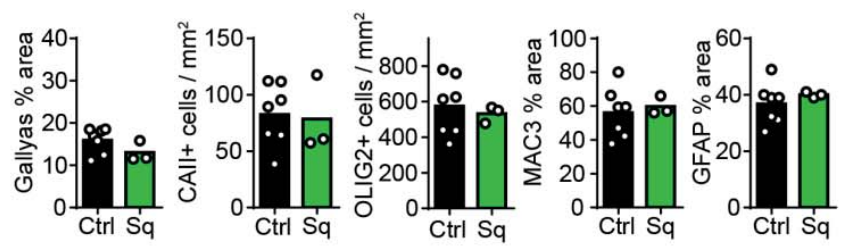


Supplementary Figure 5

A

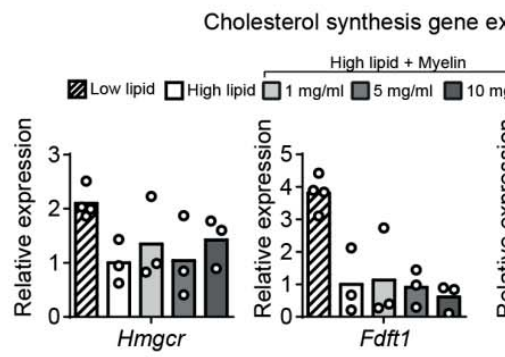

C

Cholesterol synthesis gene expression: SQScKO BMDMs $\mathrm{Hmgcr}$

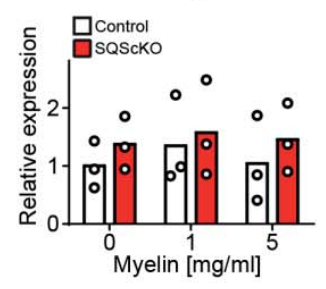

$\mathrm{E}$

$T N F$ and $I L 1 b$ expression following LPS challenge
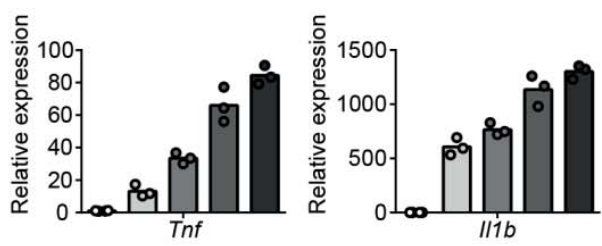

$\mathrm{H}$
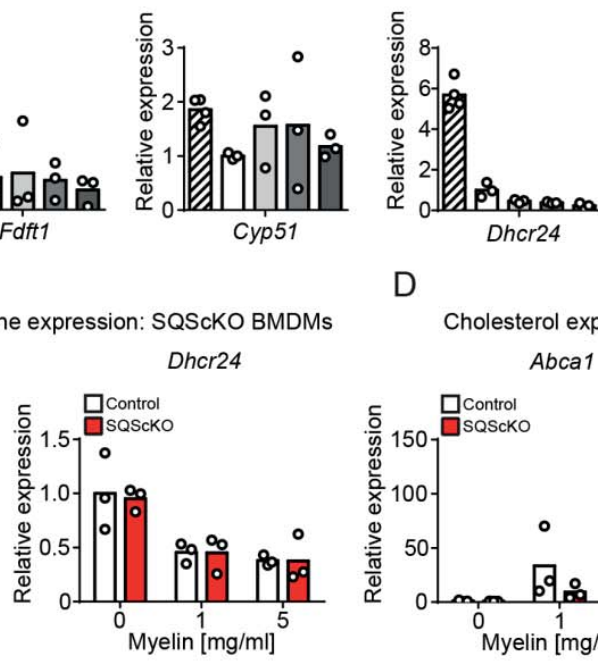

D Dhcr24

$$
\text { Abca } 1
$$

$\mathrm{F}$

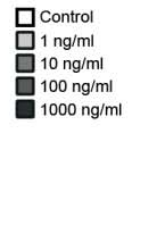

B

Ch25h expression: SQScKO BMDMs

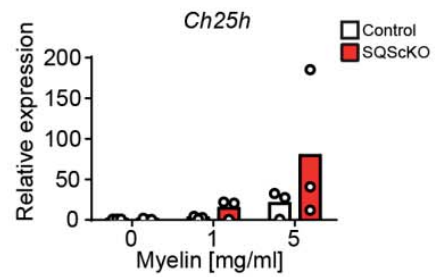

Cholesterol export gene expression: SQScKO BMDMs

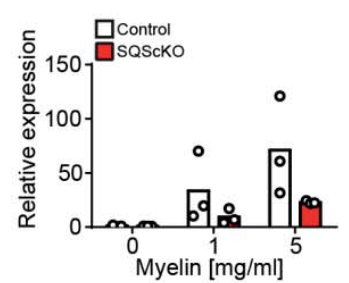

Abcg1

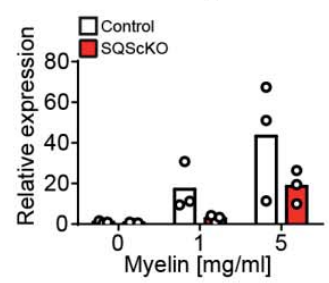

G

in vitro: LPS treatement in vitro: LPS + Myelin
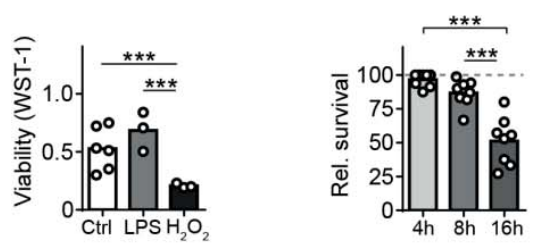

Nos2 expression: SQScKO BMDMs

ROS production: SQScKO BMDMs
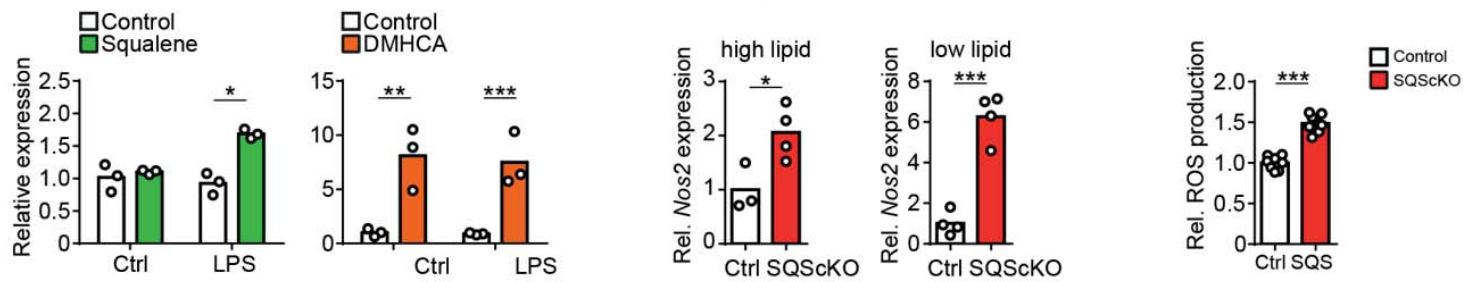

$\mathrm{K}$

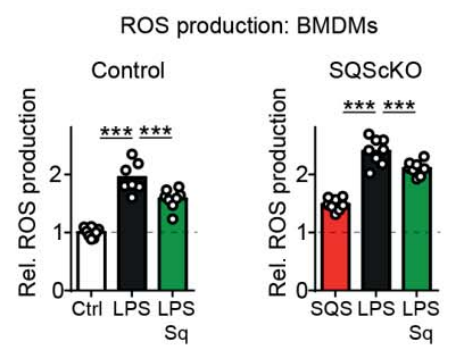




\section{Supplementary Figure 6}

A

Sterol measurement: in vivo sorted CD11b+ (Cuprizone)
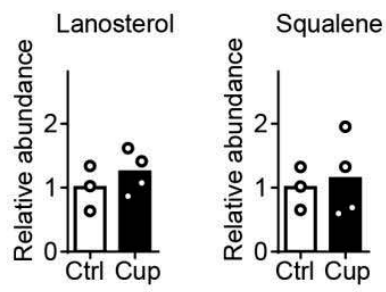

C

Sterol measurement: Cuprizone
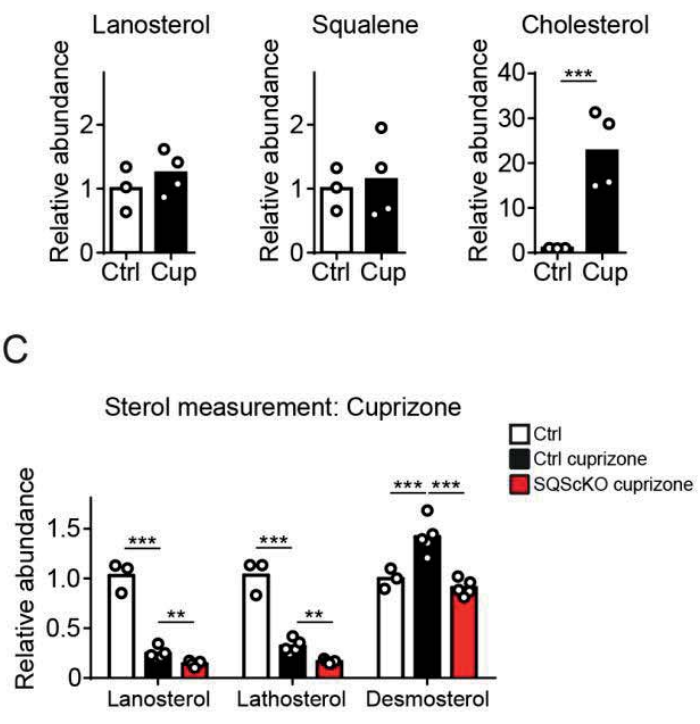

B Sterol measurement: Lysolecithin

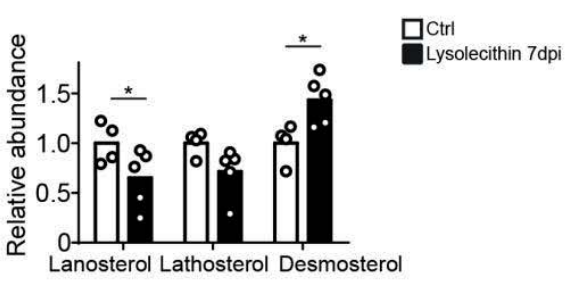

D

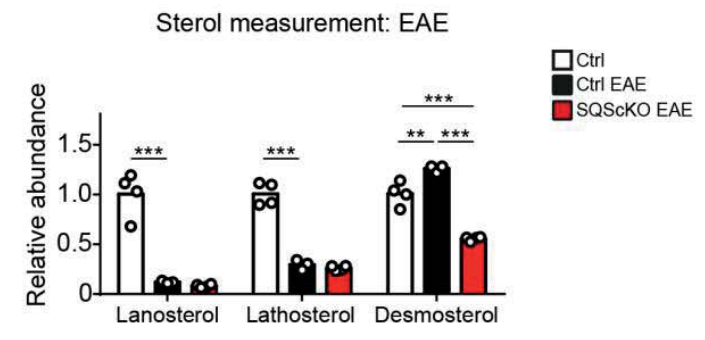

E LXR target gene expression: in vivo sorted CD11b+ cells

EAE chronic

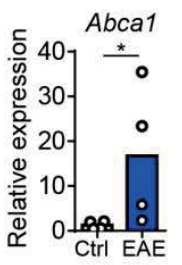

MBPcKO $12 \mathrm{mo}$.

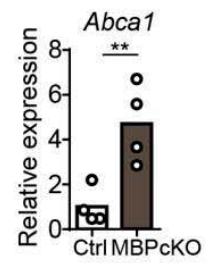

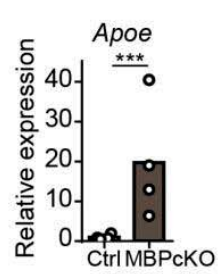

Lysolecithin 7dpi

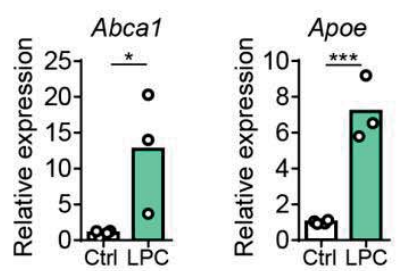

$\mathrm{F}$

Sterol measurement: EAE Squalene

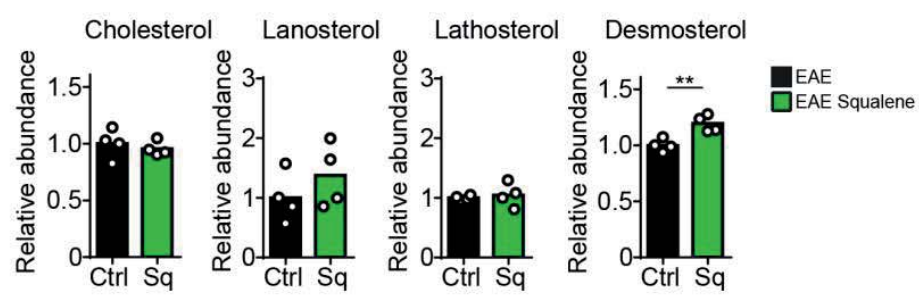




\section{Supplementary Figure 7}

A

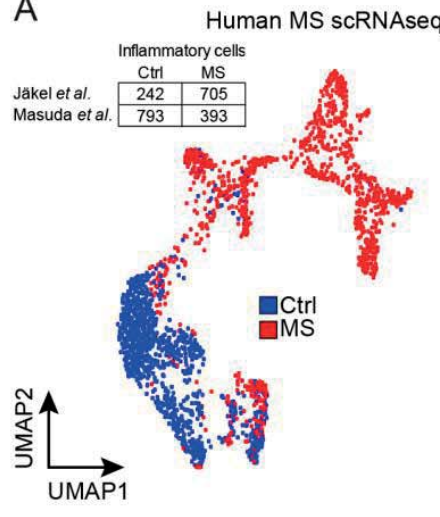

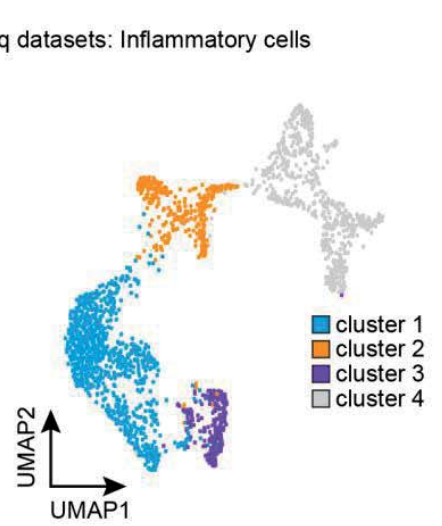

C

AIF1

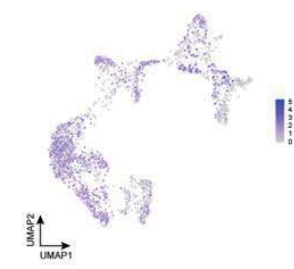

D DHCR24

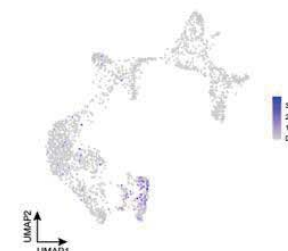

E
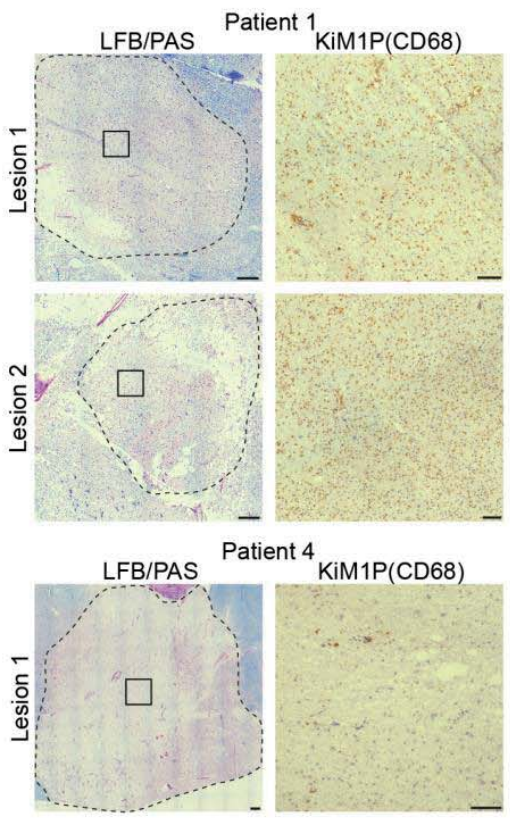

CX3CR1

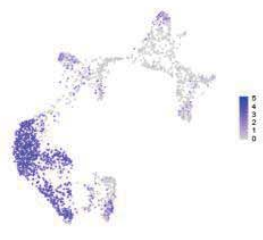

ABCA1

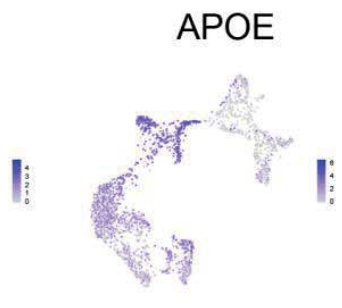

PLP1

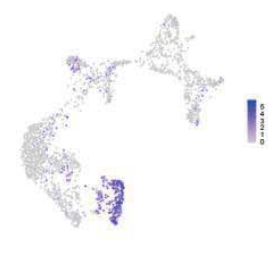

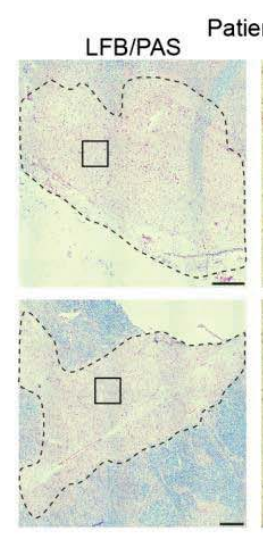

$\mathrm{F}$

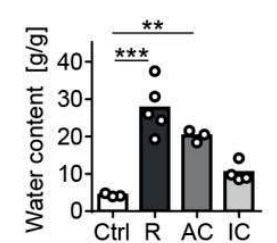

B
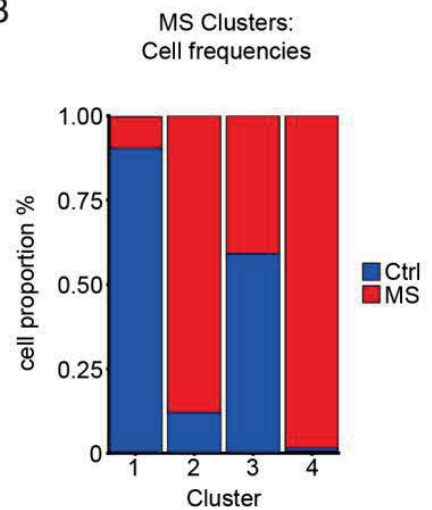

CD3

S100A4

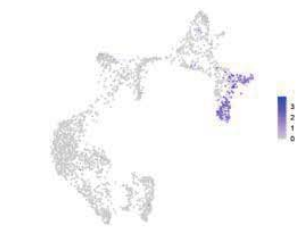

NR1H2

$\mathrm{NR} 1 \mathrm{H} 3$

Histological characterization: Human Multiple Sclerosis lesions

Patient 3 KiM1P(CD68)

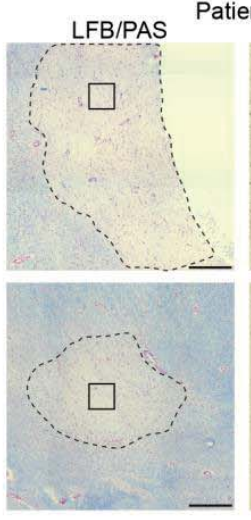

G Sterol measurement

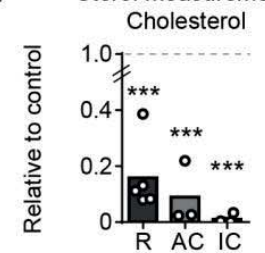




\section{Supplementary Figure 8}

A

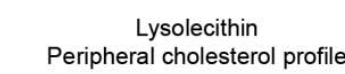

Control

宁

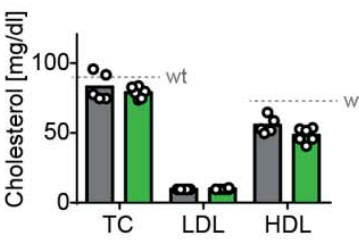

D

E

EAE 28 days

DEAE
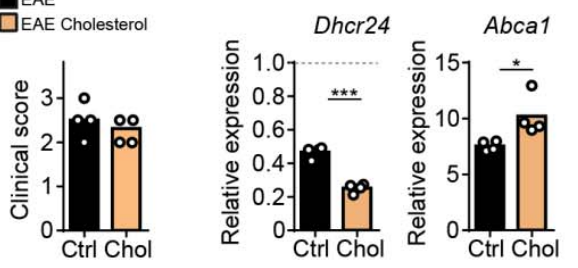

G FACS analysis: Cholesterol + IFN $\beta-1 b$ treatment

Control

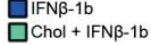
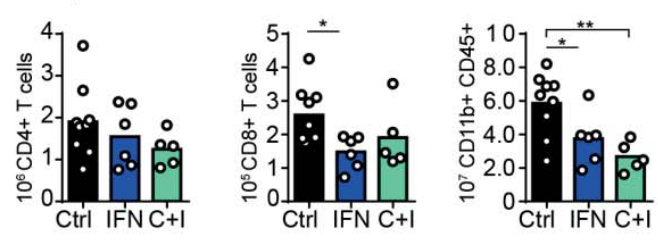

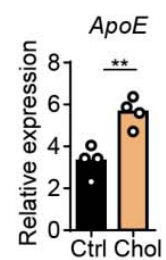

B

Lysolecithin

口Control

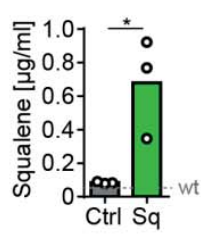

Ctrl Chol

I

Targeted inflammatory gene expression profile: In vivo sorted CD11b + cells

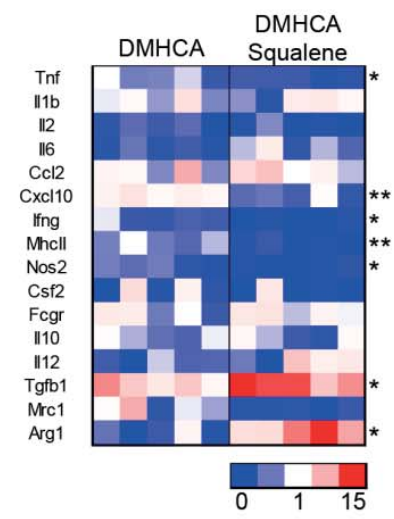

$\mathrm{H}$

C

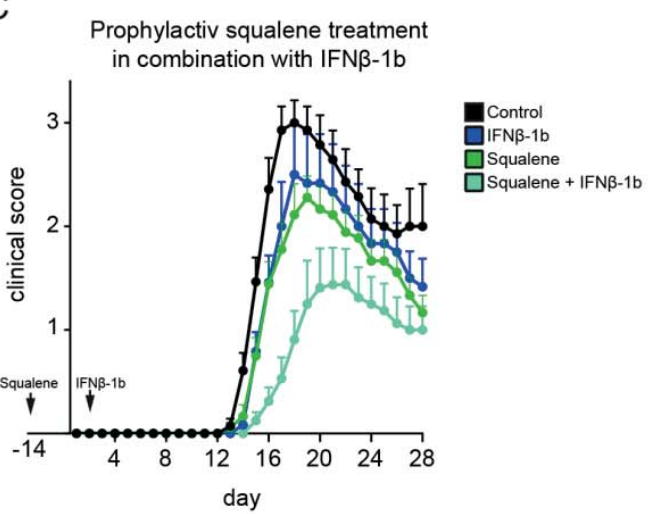

F

Prophylactiv cholesterol treatment in combination with IFN $\beta-1 b$

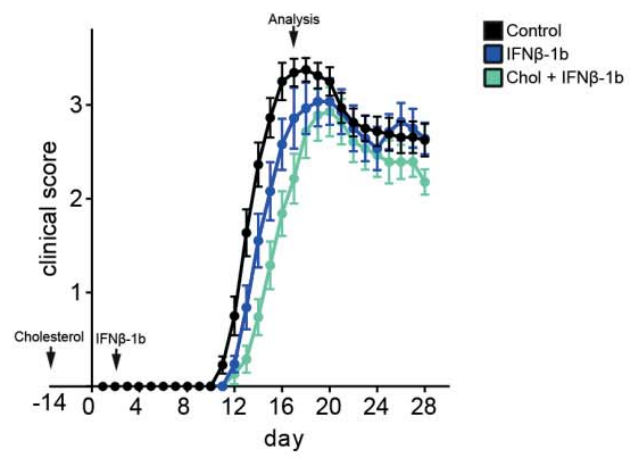

Inflammatory infiltrate : Cholesterol + IFN $\beta$ - 1 b treatment In vivo expression

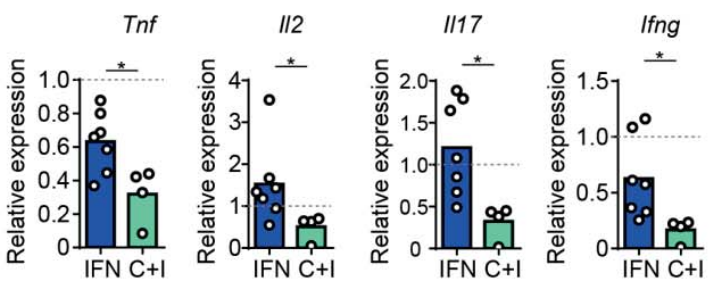




\section{Supplementary Figure 9}

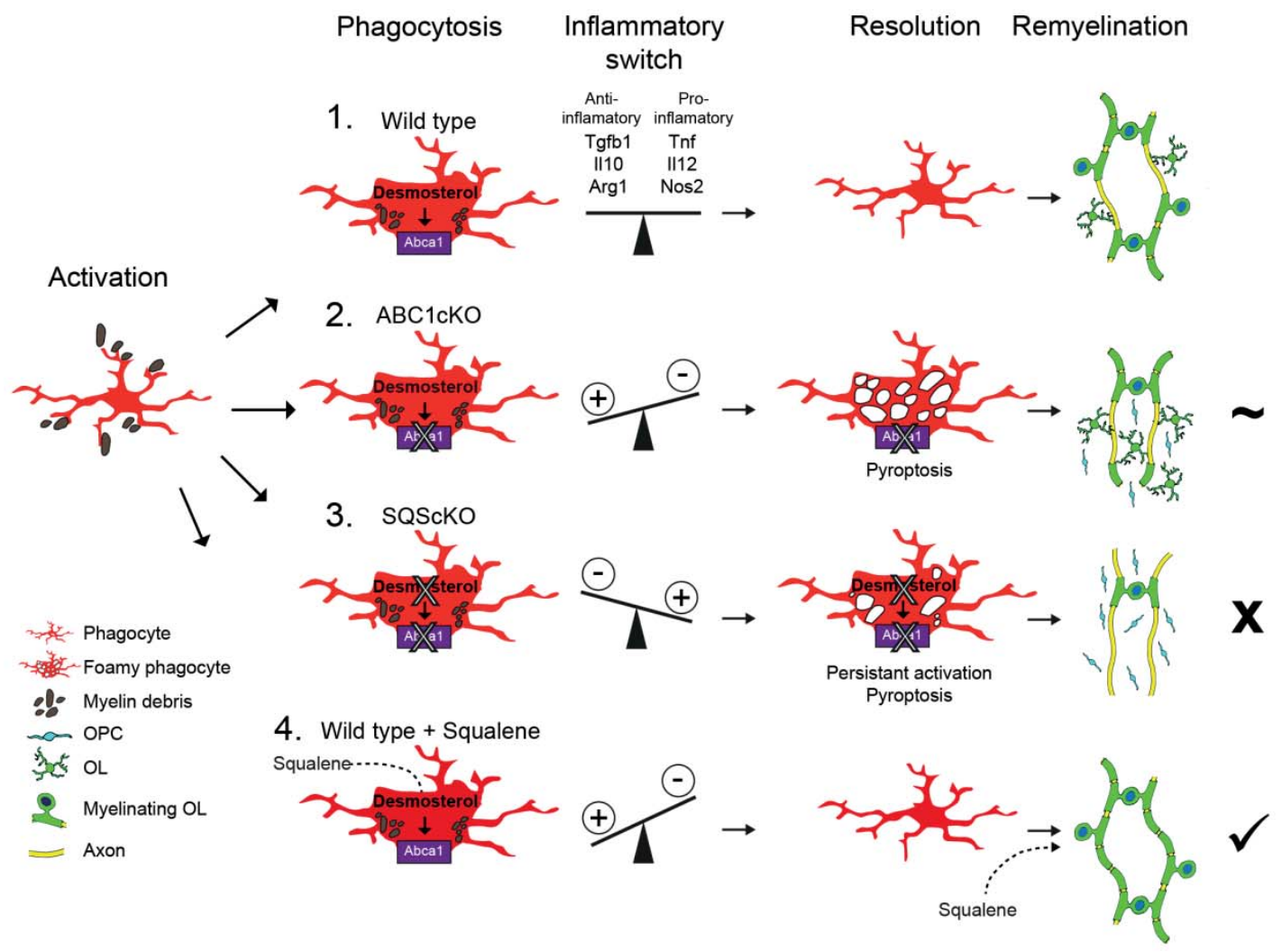




\section{Discussion}

Cholesterol in the CNS is synthesized locally and cholesterol metabolism is essentially independent of the periphery due to restricted entry of molecules at the BBB. During development, genetically induced ablation of cholesterol/lipid synthesis in glial cells, neurons or neural stem cells impairs normal brain maturation (Camargo et al., 2012; Camargo et al., 2017; Ferris et al., 2017; Funfschilling et al., 2012; Saher et al., 2005; Saito et al., 2009; van Deijk et al., 2017). By specifically eliminating cholesterol synthesis in oligodendrocytes it has been shown, that cholesterol availability is a prerequisite for developmental myelination (Saher et al., 2005). Therefore, studies targeting brain cell specific cholesterol/lipid synthesis during this critical period have revealed important insights into developmental CNS cholesterol metabolism. Several experimental observations have linked neurodegenerative diseases to altered brain cholesterol metabolism (Anchisi et al., 2012; Hung et al., 2013; Hussain et al., 2019; Valenza et al., 2015). Especially, during de- and remyelinating diseases like MS, disruption of lipid metabolism likely contributes to pathology. However, the role of cholesterol availability, the contribution of individual cell-types to CNS cholesterol metabolism and the relevance of the capability of different cell types to synthesize cholesterol during de- and remyelination are largely unknown. In my Ph.D. study, I analyzed blood brain barrier permeability during demyelination , cell type specific and tissue related cholesterol metabolism during deand remyelination, remyelination efficiency of cholesterol synthesis mutants and therapeutic potential of sterols in several MS mouse models (Berghoff et al., 2017a; Berghoff et al., 2017b).

Blood brain barrier in demyelinating diseases. It is widely accepted that cuprizone induced demyelination of the corpus callosum accompanied by pronounced gliosis occurs, while the BBB remains intact (Bakker and Ludwin, 1987; Boretius et al., 2012; Kondo et al., 1987; Matsushima and Morell, 2001). However, by applying sensitive techniques, my study showed that tight junctions are destabilized leading to increased vascular permeability (Berghoff et al., 2017b) which has been confirmed by others (Wang et al., 2020). Further, the data presented here indicate that BBB disruption is mediated by astroglial secretion of several BBB destabilizing cytokines such as IL6, IL1 $\beta$, TNF or CCL2 even before the onset of demyelination. In addition, 
influx of tracers with different biochemical properties (NaFI, EB, FITC-Dextran and BODIPY-Cholesterol) is accompanied by raised brain water content and enhanced expression and mislocalization of astrocytic AQP4, both characteristics of vasogenic edema that is also observed in MS and inflammatory models of MS (Balashov et al., 2011; Roemer et al., 2007; Stokum et al., 2016; Wolburg-Buchholz et al., 2009). It has been suggested that BBB leakage precedes white matter lesion establishment in human pathology (Cramer et al., 2015). Further, restoration of BBB integrity independent of inflammation ameliorates clinical symptoms in MS mouse models (Klicek et al., 2013; Luhder et al., 2017; Pfeiffer et al., 2011). Therefore, we suggest that early BBB dysfunction preceding demyelination might have prospective value for disease activity. In addition, our data support a concept, in which demyelinating pathologies that involve glial activation are characterized by BBB disruption.

Despite the contribution to pathology, increased vascular permeability may serve as an entry route for therapeutic compounds. In a mouse model with impaired blood brain barrier, increased lipid influx into the brain following peripheral lipid supplementation could be shown (Saher et al., 2012). In addition to hydrophilic tracers, increased influx of the lipophilic tracer BODIPY-Cholesterol in MS mouse models and therapeutic success of dietary cholesterol is observed (Berghoff et al., 2017a). However, during MS disease progression, decreased blood-brain barrier permeability and compartmentalization of inflammation has been shown (Correale et al., 2017). Therefore, it has been suggested that the relatively intact BBB limits therapy success of monoclonal antibodies (MAbs) such as natalizumab (146kDa) during chronic disease phase (Avasarala, 2017). Several strategies have been proposed to overcome BBB restricting including the use of nanoparticles, immunoliposomes, peptide vectors, and influx transporter. Combination therapy of MAbs with BBB crossing drugs, are under evaluation. In addition to size and lipophilicity, BBB penetration is restricted by the charge of the molecule, the tertiary structure and protein-binding properties. Although we provide evidence of cholesterol entry into CNS parenchyma, the exact entry route remains unknown. Cholesterol transport to peripheral organs occurs via apolipoprotein B-100 (apoB-100) containing LDL-particles. ApoB-100 with a molecular mass of $550 \mathrm{kDa}$ accounts for more than $95 \%$ of the total LDL protein mass and likely limits entry of whole lipoproteins into the 
brain (Segrest et al., 2001). In isolated endothelial cells from demyelinated corpus callosum samples, no increase of classical lipoprotein receptors such as LDLR could be detected. Nonetheless, BBB dysfunction seems to be a prerequisite for sterol/lipid entry (Saher et al., 2005). Therefore, future studies are needed, to elucidate the exact sterol entry route into the CNS taking several possible import routes into account such as paracellular influx, transcytosis and endocytosis.

Oligodendrocytes during remyelination. The here presented data support the possibility of different endogenous repair strategies during acute and chronic remyelination. During acute remyelination, oligodendroglia did not rely on autonomous cholesterol synthesis and peripheral cholesterol supplementation did not affect remyelination efficiency. It is possible, that efficient recycling of cholesterol by phagocytes following myelin clearance serves as a delivery route for cholesterol (Lavrnja et al., 2017; Olah et al., 2012), supporting oligodendrocyte differentiation and myelination (Berghoff et al., 2017a). Leaving the incorporation route of cholesterol enigmatic, we did not observe upregulation of classical LDL or VLDL receptors in oligodendrocytes that have been linked to developmental myelination (Zhao et al., 2007). Possibly, there is involvement of one, out of several other cholesterol receptor families that have not been analyzed (Lane-Donovan et al., 2014) or direct incorporation of cholesterol into myelin membranes as previously shown (Saher et al., 2012).

Conversely and in agreement with previous studies, cholesterol synthesis in oligodendroglia is indispensable during chronic remyelination (Voskuhl et al., 2019). Accordingly, peripheral cholesterol supply supported oligodendrocyte proliferation, differentiation and remyelination following chronic demyelination (Berghoff et al., 2017a). In MS, remyelination seems to be extensive during early disease phase while it often fails during chronic phase of disease (Goldschmidt et al., 2009; Nakahara, 2017). Several models have been suggested including OPC recruitment failure, reduced OPC proliferation and block of differentiation following repeated demyelination (Franklin and Ffrench-Constant, 2008). These processes are influenced by many extrinsic and intrinsic factors (Rodgers et al., 2013). In our study, increased cholesterol availability in addition to directly targeting oligodendrocytes, balances the expression of growth factors in surrounding glial cells thereby 
supporting remyelination. It might be possible that several factors following repeated demyelination and lesion chronification limit cholesterol availability and lipid synthesis in oligodendroglia. Failed upregulation of lipid synthesis genes in oligodendroglia during chronic disease phase might be associated with age, imbalanced signaling or environmental factors as previously suggested (Baror et al., 2019; Franklin, 2002; Li, 2019; O'Gorman et al., 2012). On the other hand, reduced cholesterol availability during chronic diseases phase could be caused by reduced horizontal transfer to oligodendroglia. In line with previous studies (Itoh et al., 2018), astrocytes downregulate cholesterol synthesis genes following experimental demyelination (this study). In MS white matter lesions, astrocytes eventually form a glial scar in the lesion center (Haindl et al., 2019). Although CNS protective features of glial scar formation have been described, it is possible that hypertrophic astrocytes limit cholesterol availability for oligodendroglia participating in chronification of lesions beyond physical barrier establishment. In addition, repeated demyelination and aging may impact recycling efficiency of cholesterol in phagocytes e.g. microglia. In a recent study, inefficient cholesterol efflux of aged microglia has been connected to limited remyelination supporting the possibility of sufficient cholesterol availability as important driver of remyelination (Cantuti-Castelvetri et al., 2018). Together, these data also question the use of statins in MS therapy. Although statins may have beneficial effects due to their anti-inflammatory mode of action during early disease phase (Togha et al., 2010; Vollmer et al., 2004), inhibition of cholesterol synthesis especially during chronic phase of disease can be deleterious for remyelination, as previously shown in MS mouse models (Miron et al., 2009).

Phagocyte cholesterol synthesis during demyelination. In this study, inactivation of cholesterol synthesis in CNS phagocytes induced persistent activation and cholesterol export deficiency following demyelination. By specifically targeting the cholesterol transporters $\mathrm{ABCA} 1$ and $\mathrm{ABCG} 1$ in phagocytes during demyelination, cholesterol export deficiency has been linked to limited remyelination (CantutiCastelvetri et al., 2018) that was also observed in cholesterol synthesis deficient microglia (this study). However, in contrast to primary export deficient phagocytes, we also observed persistent microgliosis simultaneously with foam cell formation following cholesterol synthesis ablation during remyelination. In line with previous 
studies, foam cell formation has been attributed to inefficient LXR activation (CantutiCastelvetri et al., 2018; Schuster et al., 2002). Integrating post squalene cholesterol synthesis and LXR activation, sterol intermediates with $L X R$ receptor ligand function have been identified in phagocytes (Shibata and Glass, 2009; Spann et al., 2012; Yang et al., 2006). In particular, lipid loading in atherosclerosis has been suggested to specifically reduce expression of the terminal cholesterol synthesis gene Dhcr24 leading to accumulation of the LXR ligand desmosterol thereby inducing cholesterol efflux and limiting inflammation (Spann et al., 2012). The data presented here support this presumptively conserved phagocyte program in mouse models of MS and active human MS lesions. Although appearance of foamy phagocytes has been thought to promote MS disease progression, our and other support a model in which phagocytes adopt a beneficial phenotype following myelin phagocytosis (Grajchen et al., 2018).

Squalene therapy in mouse models of Multiple Sclerosis. To date, a wide range of anti-inflammatory treatments are available for therapeutic management of MS (The Lancet, 2019; Torkildsen et al., 2016). However, no medication efficiently targeting remyelination is available, even though several candidates have been tested (Hooijmans et al., 2019). In this study, we identified the cholesterol precursor squalene as potential candidate for therapeutic intervention in demyelinating disease. Squalene feeds into the synthesis of the LXR ligand desmosterol in phagocytes (antiinflammatory mode of action) and directly supports myelination in oligodendrocytes (remyelinating mode of action). Although remyelination therapy targeting oligodendroglia, has become a major objective in MS (Franklin and FfrenchConstant, 2008; Rodgers et al., 2013), the overwhelming inflammatory response during acute phase of disease requires potent anti-inflammatory therapy (Berkovich, 2013; Lassmann, 2017). Thus, combining therapeutic strategies have been suggested with the attempt to avoid transition to chronic disease phase (Rodgers et al., 2013). With these data we provide evidence that lipid based therapy can be combined with first-line anti-inflammatory MS medication. Therefore, these findings highlight therapeutic potential of squalene, limiting inflammation and promoting repair after demyelinating episodes. 


\section{References}

Abbott, N.J. (2013). Blood-brain barrier structure and function and the challenges for CNS drug delivery. Journal of inherited metabolic disease 36, 437-449.

Abbott, N.J., and Friedman, A. (2012). Overview and introduction: the blood-brain barrier in health and disease. Epilepsia 53 Suppl 6, 1-6.

Abbott, N.J., Patabendige, A.A., Dolman, D.E., Yusof, S.R., and Begley, D.J. (2010). Structure and function of the blood-brain barrier. Neurobiology of disease 37, 13-25.

Abbott, N.J., Ronnback, L., and Hansson, E. (2006). Astrocyte-endothelial interactions at the bloodbrain barrier. Nature reviews. Neuroscience 7, 41-53.

Albers, J.J., Tollefson, J.H., Wolfbauer, G., and Albright, R.E., Jr. (1992). Cholesteryl ester transfer protein in human brain. International journal of clinical \& laboratory research 21, 264-266.

Alvarez, J.I., Cayrol, R., and Prat, A. (2011a). Disruption of central nervous system barriers in multiple sclerosis. Biochimica et biophysica acta 1812, 252-264.

Alvarez, J.I., Dodelet-Devillers, A., Kebir, H., Ifergan, I., Fabre, P.J., Terouz, S., Sabbagh, M., Wosik, K., Bourbonniere, L., Bernard, M., et al. (2011b). The Hedgehog Pathway Promotes Blood-Brain Barrier Integrity and CNS Immune Quiescence. Science 334, 1727-1731.

Alvarez, J.I., Katayama, T., and Prat, A. (2013). Glial influence on the blood brain barrier. Glia 61, 1939-1958.

Anchisi, L., Dessi, S., Pani, A., and Mandas, A. (2012). Cholesterol homeostasis: a key to prevent or slow down neurodegeneration. Frontiers in physiology 3, 486.

Andreone, B.J., Chow, B.W., Tata, A., Lacoste, B., Ben-Zvi, A., Bullock, K., Deik, A.A., Ginty, D.D., Clish, C.B., and Gu, C. (2017). Blood-Brain Barrier Permeability Is Regulated by Lipid TransportDependent Suppression of Caveolae-Mediated Transcytosis. Neuron 94, 581-594 e585.

Argaw, A.T., Asp, L., Zhang, J., Navrazhina, K., Pham, T., Mariani, J.N., Mahase, S., Dutta, D.J., Seto, J., Kramer, E.G., et al. (2012). Astrocyte-derived VEGF-A drives blood-brain barrier disruption in CNS inflammatory disease. The Journal of clinical investigation 122, 2454-2468.

Argaw, A.T., Gurfein, B.T., Zhang, Y., Zameer, A., and John, G.R. (2009). VEGF-mediated disruption of endothelial CLN-5 promotes blood-brain barrier breakdown. Proceedings of the National Academy of Sciences of the United States of America 106, 1977-1982.

Armulik, A., Genove, G., Mae, M., Nisancioglu, M.H., Wallgard, E., Niaudet, C., He, L., Norlin, J., Lindblom, P., Strittmatter, K., et al. (2010). Pericytes regulate the blood-brain barrier. Nature 468, 557561.

Avasarala, J. (2017). It's Time For Combination Therapies: in Multiple Sclerosis. Innovations in clinical neuroscience $14,28-30$.

Baeten, K.M., and Akassoglou, K. (2011). Extracellular matrix and matrix receptors in blood-brain barrier formation and stroke. Developmental neurobiology 71, 1018-1039.

Bakker, D.A., and Ludwin, S.K. (1987). Blood-brain barrier permeability during Cuprizone-induced demyelination. Implications for the pathogenesis of immune-mediated demyelinating diseases. Journal of the neurological sciences $78,125-137$.

Balashov, K.E., Aung, L.L., Dhib-Jalbut, S., and Keller, I.A. (2011). Acute multiple sclerosis lesion: conversion of restricted diffusion due to vasogenic edema. Journal of neuroimaging : official journal of the American Society of Neuroimaging 21, 202-204. 
Balazs, Z., Panzenboeck, U., Hammer, A., Sovic, A., Quehenberger, O., Malle, E., and Sattler, W. (2004). Uptake and transport of high-density lipoprotein (HDL) and HDL-associated alpha-tocopherol by an in vitro blood-brain barrier model. Journal of neurochemistry 89, 939-950.

Baranowski, M. (2008). Biological role of liver $X$ receptors. Journal of physiology and pharmacology : an official journal of the Polish Physiological Society 59 Suppl 7, 31-55.

Barnett, M.H., and Prineas, J.W. (2004). Relapsing and remitting multiple sclerosis: pathology of the newly forming lesion. Annals of neurology 55, 458-468.

Baror, R., Neumann, B., Segel, M., Chalut, K.J., Fancy, S.P.J., Schafer, D.P., and Franklin, R.J.M. (2019). Transforming growth factor-beta renders ageing microglia inhibitory to oligodendrocyte generation by CNS progenitors. Glia 67, 1374-1384.

Bazzoni, G., and Dejana, E. (2004). Endothelial cell-to-cell junctions: molecular organization and role in vascular homeostasis. Physiological reviews $84,869-901$.

Behan, P.O., and Chaudhuri, A. (2014). EAE is not a useful model for demyelinating disease. Multiple sclerosis and related disorders 3, 565-574.

Bergersen, L.H. (2015). Lactate transport and signaling in the brain: potential therapeutic targets and roles in body-brain interaction. Journal of cerebral blood flow and metabolism : official journal of the International Society of Cerebral Blood Flow and Metabolism 35, 176-185.

Berghoff, S.A., Duking, T., Spieth, L., Winchenbach, J., Stumpf, S.K., Gerndt, N., Kusch, K., Ruhwedel, T., Mobius, W., and Saher, G. (2017a). Blood-brain barrier hyperpermeability precedes demyelination in the cuprizone model. Acta neuropathologica communications 5, 94.

Berghoff, S.A., Gerndt, N., Winchenbach, J., Stumpf, S.K., Hosang, L., Odoardi, F., Ruhwedel, T., Böhler, C., Barrette, B., Stassart, R., et al. (2017b). Dietary cholesterol promotes repair of demyelinated lesions in the adult brain. Nature communications 8, 14241.

Berkovich, R. (2013). Treatment of acute relapses in multiple sclerosis. Neurotherapeutics : the journal of the American Society for Experimental NeuroTherapeutics 10, 97-105.

Biardi, L., Sreedhar, A., Zokaei, A., Vartak, N.B., Bozeat, R.L., Shackelford, J.E., Keller, G.A., and Krisans, S.K. (1994). Mevalonate kinase is predominantly localized in peroxisomes and is defective in patients with peroxisome deficiency disorders. The Journal of biological chemistry 269, 1197-1205.

Bieber, A.J., Kerr, S., and Rodriguez, M. (2003). Efficient central nervous system remyelination requires T cells. Annals of neurology 53, 680-684.

Billiau, A., and Matthys, P. (2001). Modes of action of Freund's adjuvants in experimental models of autoimmune diseases. Journal of leukocyte biology 70, 849-860.

Bjelobaba, I., Begovic-Kupresanin, V., Pekovic, S., and Lavrnja, I. (2018). Animal models of multiple sclerosis: Focus on experimental autoimmune encephalomyelitis. Journal of neuroscience research 96, 1021-1042.

Bjorkhem, I., Leoni, V., and Meaney, S. (2010). Genetic connections between neurological disorders and cholesterol metabolism. Journal of lipid research 51, 2489-2503.

Bjorkhem, I., Leoni, V., and Svenningsson, P. (2019). On the fluxes of side-chain oxidized oxysterols across blood-brain and blood-CSF barriers and origin of these steroids in CSF (Review). The Journal of steroid biochemistry and molecular biology $188,86-89$. 
Bjorkhem, I., Lutjohann, D., Diczfalusy, U., Stahle, L., Ahlborg, G., and Wahren, J. (1998). Cholesterol homeostasis in human brain: turnover of 24S-hydroxycholesterol and evidence for a cerebral origin of most of this oxysterol in the circulation. Journal of lipid research 39, 1594-1600.

Bjorkhem, I., and Meaney, S. (2004). Brain cholesterol: long secret life behind a barrier. Arteriosclerosis, thrombosis, and vascular biology 24, 806-815.

Bloch, K. (1965). The biological synthesis of cholesterol. Science 150, 19-28.

Boadu, E., and Francis, G.A. (2006). The role of vesicular transport in ABCA1-dependent lipid efflux and its connection with NPC pathways. Journal of molecular medicine 84, 266-275.

Boadu, E., Nelson, R.C., and Francis, G.A. (2012). ABCA1-dependent mobilization of lysosomal cholesterol requires functional Niemann-Pick C2 but not Niemann-Pick $\mathrm{C} 1$ protein. Biochimica et biophysica acta 1821, 396-404.

Bohlen, C.J., Bennett, F.C., Tucker, A.F., Collins, H.Y., Mulinyawe, S.B., and Barres, B.A. (2017). Diverse Requirements for Microglial Survival, Specification, and Function Revealed by DefinedMedium Cultures. Neuron 94, 759-773 e758.

Boretius, S., Escher, A., Dallenga, T., Wrzos, C., Tammer, R., Bruck, W., Nessler, S., Frahm, J., and Stadelmann, C. (2012). Assessment of lesion pathology in a new animal model of MS by multiparametric MRI and DTI. Neurolmage 59, 2678-2688.

Boyd, A., Zhang, H., and Williams, A. (2013). Insufficient OPC migration into demyelinated lesions is a cause of poor remyelination in MS and mouse models. Acta neuropathologica 125, 841-859.

Breij, E.C., Brink, B.P., Veerhuis, R., van den Berg, C., Vloet, R., Yan, R., Dijkstra, C.D., van der Valk, P., and Bo, L. (2008). Homogeneity of active demyelinating lesions in established multiple sclerosis. Annals of neurology 63, 16-25.

Breitling, R., and Krisans, S.K. (2002). A second gene for peroxisomal HMG-CoA reductase? A genomic reassessment. Journal of lipid research 43, 2031-2036.

Brown, M.S., and Goldstein, J.L. (1979). Receptor-mediated endocytosis: insights from the lipoprotein receptor system. Proceedings of the National Academy of Sciences of the United States of America $76,3330-3337$.

Brown, R.A., Narayanan, S., and Arnold, D.L. (2014). Imaging of repeated episodes of demyelination and remyelination in multiple sclerosis. Neurolmage. Clinical 6, 20-25.

Buhaescu, I., and Izzedine, H. (2007). Mevalonate pathway: a review of clinical and therapeutical implications. Clinical biochemistry 40, 575-584.

Buschmann, J.P., Berger, K., Awad, H., Clarner, T., Beyer, C., and Kipp, M. (2012). Inflammatory response and chemokine expression in the white matter corpus callosum and gray matter cortex region during cuprizone-induced demyelination. Journal of molecular neuroscience : MN 48, 66-76.

Calabrese, M., Filippi, M., and Gallo, P. (2010). Cortical lesions in multiple sclerosis. Nature reviews. Neurology 6, 438-444.

Camargo, N., Brouwers, J.F., Loos, M., Gutmann, D.H., Smit, A.B., and Verheijen, M.H. (2012). Highfat diet ameliorates neurological deficits caused by defective astrocyte lipid metabolism. FASEB journal : official publication of the Federation of American Societies for Experimental Biology 26, 43024315. 
Camargo, N., Goudriaan, A., van Deijk, A.F., Otte, W.M., Brouwers, J.F., Lodder, H., Gutmann, D.H., Nave, K.A., Dijkhuizen, R.M., Mansvelder, H.D., et al. (2017). Oligodendroglial myelination requires astrocyte-derived lipids. PLoS biology 15, e1002605.

Camire, R.B., Beaulac, H.J., and Willis, C.L. (2015). Transitory loss of glia and the subsequent modulation in inflammatory cytokines/chemokines regulate paracellular claudin-5 expression in endothelial cells. Journal of neuroimmunology 284, 57-66.

Canellas, A.R., Gols, A.R., Izquierdo, J.R., Subirana, M.T., and Gairin, X.M. (2007). Idiopathic inflammatory-demyelinating diseases of the central nervous system. Neuroradiology 49, 393-409.

Cantagrel, V., and Lefeber, D.J. (2011). From glycosylation disorders to dolichol biosynthesis defects: a new class of metabolic diseases. Journal of inherited metabolic disease 34, 859-867.

Cantuti-Castelvetri, L., Fitzner, D., Bosch-Queralt, M., Weil, M.T., Su, M., Sen, P., Ruhwedel, T., Mitkovski, M., Trendelenburg, G., Lutjohann, D., et al. (2018). Defective cholesterol clearance limits remyelination in the aged central nervous system. Science 359, 684-688.

Carlton, W.W. (1967). Studies on the induction of hydrocephalus and spongy degeneration by cuprizone feeding and attempts to antidote the toxicity. Life sciences 6, 11-19.

Carstea, E.D., Morris, J.A., Coleman, K.G., Loftus, S.K., Zhang, D., Cummings, C., Gu, J., Rosenfeld, M.A., Pavan, W.J., Krizman, D.B., et al. (1997). Niemann-Pick C1 disease gene: homology to mediators of cholesterol homeostasis. Science 277, 228-231.

Cerqueira, N.M., Oliveira, E.F., Gesto, D.S., Santos-Martins, D., Moreira, C., Moorthy, H.N., Ramos, M.J., and Fernandes, P.A. (2016). Cholesterol Biosynthesis: A Mechanistic Overview. Biochemistry 55, 5483-5506.

Chakrabarti, R.S., Ingham, S.A., Kozlitina, J., Gay, A., Cohen, J.C., Radhakrishnan, A., and Hobbs, H.H. (2017). Variability of cholesterol accessibility in human red blood cells measured using a bacterial cholesterol-binding toxin. eLife 6.

Chang, A., Tourtellotte, W.W., Rudick, R., and Trapp, B.D. (2002). Premyelinating oligodendrocytes in chronic lesions of multiple sclerosis. The New England journal of medicine 346, 165-173.

Chang, T.Y., Li, B.L., Chang, C.C., and Urano, Y. (2009). Acyl-coenzyme A:cholesterol acyltransferases. American journal of physiology. Endocrinology and metabolism 297, E1-9.

Chari, D.M. (2007). Remyelination in multiple sclerosis. International review of neurobiology 79, 589620.

Charlton-Menys, V., and Durrington, P.N. (2007). Squalene synthase inhibitors : clinical pharmacology and cholesterol-lowering potential. Drugs 67, 11-16.

Chirackal Manavalan, A.P., Kober, A., Metso, J., Lang, I., Becker, T., Hasslitzer, K., Zandl, M., Fanaee-Danesh, E., Pippal, J.B., Sachdev, V., et al. (2014). Phospholipid transfer protein is expressed in cerebrovascular endothelial cells and involved in high density lipoprotein biogenesis and remodeling at the blood-brain barrier. The Journal of biological chemistry 289, 4683-4698.

Chiry, O., Pellerin, L., Monnet-Tschudi, F., Fishbein, W.N., Merezhinskaya, N., Magistretti, P.J., and Clarke, S. (2006). Expression of the monocarboxylate transporter MCT1 in the adult human brain cortex. Brain research 1070, 65-70.

Chu, B.B., Liao, Y.C., Qi, W., Xie, C., Du, X., Wang, J., Yang, H., Miao, H.H., Li, B.L., and Song, B.L. (2015). Cholesterol transport through lysosome-peroxisome membrane contacts. Cell 161, 291-306. 
Clarner, T., Janssen, K., Nellessen, L., Stangel, M., Skripuletz, T., Krauspe, B., Hess, F.M., Denecke, B., Beutner, C., Linnartz-Gerlach, B., et al. (2015). CXCL10 triggers early microglial activation in the cuprizone model. Journal of immunology 194, 3400-3413.

Collaborators, G.B.D.M.S. (2019). Global, regional, and national burden of multiple sclerosis 19902016: a systematic analysis for the Global Burden of Disease Study 2016. The Lancet. Neurology 18, 269-285.

Constantinescu, C.S., Farooqi, N., O'Brien, K., and Gran, B. (2011). Experimental autoimmune encephalomyelitis (EAE) as a model for multiple sclerosis (MS). British journal of pharmacology 164, $1079-1106$.

Coomber, B.L., and Stewart, P.A. (1985). Morphometric analysis of CNS microvascular endothelium. Microvascular research $30,99-115$.

Correale, J., Gaitan, M.I., Ysrraelit, M.C., and Fiol, M.P. (2017). Progressive multiple sclerosis: from pathogenic mechanisms to treatment. Brain : a journal of neurology 140, 527-546.

Courtney, R., and Landreth, G.E. (2016). LXR Regulation of Brain Cholesterol: From Development to Disease. Trends in endocrinology and metabolism: TEM 27, 404-414.

Cramer, S.P., Modvig, S., Simonsen, H.J., Frederiksen, J.L., and Larsson, H.B. (2015). Permeability of the blood-brain barrier predicts conversion from optic neuritis to multiple sclerosis. Brain : a journal of neurology 138, 2571-2583.

Cramer, S.P., Simonsen, H., Frederiksen, J.L., Rostrup, E., and Larsson, H.B. (2014). Abnormal blood-brain barrier permeability in normal appearing white matter in multiple sclerosis investigated by MRI. Neurolmage. Clinical 4, 182-189.

Crawford, A.H., Tripathi, R.B., Richardson, W.D., and Franklin, R.J.M. (2016). Developmental Origin of Oligodendrocyte Lineage Cells Determines Response to Demyelination and Susceptibility to AgeAssociated Functional Decline. Cell reports 15, 761-773.

da Fonseca, A.C., Matias, D., Garcia, C., Amaral, R., Geraldo, L.H., Freitas, C., and Lima, F.R. (2014). The impact of microglial activation on blood-brain barrier in brain diseases. Frontiers in cellular neuroscience 8,362 .

Daneman, R., Agalliu, D., Zhou, L., Kuhnert, F., Kuo, C.J., and Barres, B.A. (2009). Wnt/beta-catenin signaling is required for CNS, but not non-CNS, angiogenesis. Proceedings of the National Academy of Sciences of the United States of America 106, 641-646.

Daneman, R., Zhou, L., Agalliu, D., Cahoy, J.D., Kaushal, A., and Barres, B.A. (2010). The mouse blood-brain barrier transcriptome: a new resource for understanding the development and function of brain endothelial cells. PloS one 5, e13741.

Davalos, D., Baeten, K.M., Whitney, M.A., Mullins, E.S., Friedman, B., Olson, E.S., Ryu, J.K., Smirnoff, D.S., Petersen, M.A., Bedard, C., et al. (2014). Early detection of thrombin activity in neuroinflammatory disease. Annals of neurology $75,303-308$.

DeBose-Boyd, R.A. (2008). Feedback regulation of cholesterol synthesis: sterol-accelerated ubiquitination and degradation of HMG CoA reductase. Cell research 18, 609-621.

Dietschy, J.M. (2009). Central nervous system: cholesterol turnover, brain development and neurodegeneration. Biological chemistry 390, 287-293.

Dietschy, J.M., and Turley, S.D. (2004). Thematic review series: brain Lipids. Cholesterol metabolism in the central nervous system during early development and in the mature animal. Journal of lipid research $45,1375-1397$. 
Doan, V., Kleindienst, A.M., McMahon, E.J., Long, B.R., Matsushima, G.K., and Taylor, L.C. (2013). Abbreviated exposure to cuprizone is sufficient to induce demyelination and oligodendrocyte loss. Journal of neuroscience research 91, 363-373.

Dobolyi, A., Vincze, C., Pal, G., and Lovas, G. (2012). The Neuroprotective Functions of Transforming Growth Factor Beta Proteins. International journal of molecular sciences 13, 8219-8258.

Dobson, R., and Giovannoni, G. (2019). Multiple sclerosis - a review. European journal of neurology 26, 27-40.

Dombrowski, Y., O'Hagan, T., Dittmer, M., Penalva, R., Mayoral, S.R., Bankhead, P., Fleville, S., Eleftheriadis, G., Zhao, C., Naughton, M., et al. (2017). Regulatory T cells promote myelin regeneration in the central nervous system. Nature neuroscience 20,674-680.

Doucette, J.R., Jiao, R., and Nazarali, A.J. (2010). Age-related and cuprizone-induced changes in myelin and transcription factor gene expression and in oligodendrocyte cell densities in the rostral corpus callosum of mice. Cellular and molecular neurobiology 30, 607-629.

Duncan, I.D., Marik, R.L., Broman, A.T., and Heidari, M. (2017). Thin myelin sheaths as the hallmark of remyelination persist over time and preserve axon function. Proceedings of the National Academy of Sciences of the United States of America 114, E9685-E9691.

Duncan, I.D., Radcliff, A.B., Heidari, M., Kidd, G., August, B.K., and Wierenga, L.A. (2018). The adult oligodendrocyte can participate in remyelination. Proceedings of the National Academy of Sciences of the United States of America 115, E11807-E11816.

Efendi, H. (2015). Clinically Isolated Syndromes: Clinical Characteristics, Differential Diagnosis, and Management. Noro psikiyatri arsivi 52, S1-S11.

Ehrlich, P. (1885). Das Sauerstoff-Bedürfniss des Organismus: eine farbenanalytische Studie. (August Hirschwald).

Endo, A. (2010). A historical perspective on the discovery of statins. Proceedings of the Japan Academy. Series B, Physical and biological sciences $86,484-493$.

Engelhardt, B. (2006). Molecular mechanisms involved in T cell migration across the blood-brain barrier. Journal of neural transmission 113, 477-485.

Engelhardt, B., Carare, R.O., Bechmann, I., Flugel, A., Laman, J.D., and Weller, R.O. (2016). Vascular, glial, and lymphatic immune gateways of the central nervous system. Acta neuropathologica 132, 317-338.

Engelhardt, B., and Coisne, C. (2011). Fluids and barriers of the CNS establish immune privilege by confining immune surveillance to a two-walled castle moat surrounding the CNS castle. Fluids and barriers of the CNS 8,4 .

Espenshade, P.J. (2006). SREBPs: sterol-regulated transcription factors. Journal of cell science 119, 973-976.

Evans, W.R., and Hendriksz, C.J. (2017). Niemann-Pick type C disease - the tip of the iceberg? A review of neuropsychiatric presentation, diagnosis and treatment. BJPsych bulletin 41, 109-114.

Fabis, M.J., Scott, G.S., Kean, R.B., Koprowski, H., and Hooper, D.C. (2007). Loss of blood-brain barrier integrity in the spinal cord is common to experimental allergic encephalomyelitis in knockout mouse models. Proceedings of the National Academy of Sciences of the United States of America 104, 5656-5661. 
Falcao, A.M., van Bruggen, D., Marques, S., Meijer, M., Jakel, S., Agirre, E., Samudyata, Floriddia, E.M., Vanichkina, D.P., Ffrench-Constant, C., et al. (2018). Disease-specific oligodendrocyte lineage cells arise in multiple sclerosis. Nature medicine 24, 1837-1844.

Faust, J.R., Goldstein, J.L., and Brown, M.S. (1979). Synthesis of ubiquinone and cholesterol in human fibroblasts: regulation of a branched pathway. Archives of biochemistry and biophysics 192, 86-99.

Faust, P.L., and Kovacs, W.J. (2014). Cholesterol biosynthesis and ER stress in peroxisome deficiency. Biochimie 98, 75-85.

Fazio, S., Linton, M.F., and Swift, L.L. (2000). The cell biology and physiologic relevance of ApoE recycling. Trends in cardiovascular medicine 10, 23-30.

Fenstermacher, J., Gross, P., Sposito, N., Acuff, V., Pettersen, S., and Gruber, K. (1988). Structural and functional variations in capillary systems within the brain. Annals of the New York Academy of Sciences 529, 21-30.

Ferrari, C.C., Depino, A.M., Prada, F., Muraro, N., Campbell, S., Podhajcer, O., Perry, V.H., Anthony, D.C., and Pitossi, F.J. (2004). Reversible demyelination, blood-brain barrier breakdown, and pronounced neutrophil recruitment induced by chronic IL-1 expression in the brain. The American journal of pathology $165,1827-1837$.

Ferris, H.A., Perry, R.J., Moreira, G.V., Shulman, G.I., Horton, J.D., and Kahn, C.R. (2017). Loss of astrocyte cholesterol synthesis disrupts neuronal function and alters whole-body metabolism.

Proceedings of the National Academy of Sciences of the United States of America 114, 1189-1194.

Figley, C.R., and Stroman, P.W. (2011). The role(s) of astrocytes and astrocyte activity in neurometabolism, neurovascular coupling, and the production of functional neuroimaging signals. The European journal of neuroscience 33, 577-588.

Filippi, M., Bar-Or, A., Piehl, F., Preziosa, P., Solari, A., Vukusic, S., and Rocca, M.A. (2018). Multiple sclerosis. Nature reviews. Disease primers 4, 43.

Franklin, R.J. (2002). Why does remyelination fail in multiple sclerosis? Nature reviews. Neuroscience 3, 705-714.

Franklin, R.J., and Ffrench-Constant, C. (2008). Remyelination in the CNS: from biology to therapy. Nature reviews. Neuroscience 9, 839-855.

Franklin, R.J., ffrench-Constant, C., Edgar, J.M., and Smith, K.J. (2012). Neuroprotection and repair in multiple sclerosis. Nature reviews. Neurology 8, 624-634.

Frischer, J.M., Weigand, S.D., Guo, Y., Kale, N., Parisi, J.E., Pirko, I., Mandrekar, J., Bramow, S., Metz, I., Bruck, W., et al. (2015). Clinical and pathological insights into the dynamic nature of the white matter multiple sclerosis plaque. Annals of neurology 78, 710-721.

Fujimoto, T., and Parton, R.G. (2011). Not just fat: the structure and function of the lipid droplet. Cold Spring Harbor perspectives in biology 3.

Funfschilling, U., Jockusch, W.J., Sivakumar, N., Mobius, W., Corthals, K., Li, S., Quintes, S., Kim, Y., Schaap, I.A., Rhee, J.S., et al. (2012). Critical time window of neuronal cholesterol synthesis during neurite outgrowth. The Journal of neuroscience : the official journal of the Society for Neuroscience 32, 7632-7645.

Glatigny, S., and Bettelli, E. (2018). Experimental Autoimmune Encephalomyelitis (EAE) as Animal Models of Multiple Sclerosis (MS). Cold Spring Harbor perspectives in medicine 8. 
Gold, R., Linington, C., and Lassmann, H. (2006). Understanding pathogenesis and therapy of multiple sclerosis via animal models: 70 years of merits and culprits in experimental autoimmune encephalomyelitis research. Brain : a journal of neurology 129, 1953-1971.

Goldberg, J., Daniel, M., van Heuvel, Y., Victor, M., Beyer, C., Clarner, T., and Kipp, M. (2013). Shortterm cuprizone feeding induces selective amino acid deprivation with concomitant activation of an integrated stress response in oligodendrocytes. Cellular and molecular neurobiology 33, 1087-1098.

Goldenberg, M.M. (2012). Multiple sclerosis review. P \& T : a peer-reviewed journal for formulary management 37, 175-184.

Goldschmidt, T., Antel, J., Konig, F.B., Bruck, W., and Kuhlmann, T. (2009). Remyelination capacity of the MS brain decreases with disease chronicity. Neurology 72, 1914-1921.

Goldstein, J.L., and Brown, M.S. (1990). Regulation of the mevalonate pathway. Nature 343, 425-430.

Goldstein, J.L., and Brown, M.S. (2009). The LDL receptor. Arteriosclerosis, thrombosis, and vascular biology 29, 431-438.

Goldstein, J.L., DeBose-Boyd, R.A., and Brown, M.S. (2006). Protein sensors for membrane sterols. Cell 124, 35-46.

Grabacka, M., Pierzchalska, M., Dean, M., and Reiss, K. (2016). Regulation of Ketone Body Metabolism and the Role of PPARalpha. International journal of molecular sciences 17.

Grajchen, E., Hendriks, J.J.A., and Bogie, J.F.J. (2018). The physiology of foamy phagocytes in multiple sclerosis. Acta neuropathologica communications 6, 124.

Greene, C., Hanley, N., and Campbell, M. (2019). Claudin-5: gatekeeper of neurological function. Fluids and barriers of the CNS 16, 3.

Gudi, V., Gingele, S., Skripuletz, T., and Stangel, M. (2014). Glial response during cuprizone-induced de- and remyelination in the CNS: lessons learned. Frontiers in cellular neuroscience $8,73$.

Gudi, V., Moharregh-Khiabani, D., Skripuletz, T., Koutsoudaki, P.N., Kotsiari, A., Skuljec, J., Trebst, C., and Stangel, M. (2009). Regional differences between grey and white matter in cuprizone induced demyelination. Brain research 1283, 127-138.

Hagan, N., and Ben-Zvi, A. (2015). The molecular, cellular, and morphological components of bloodbrain barrier development during embryogenesis. Seminars in cell \& developmental biology 38, 7-15.

Haindl, M.T., Kock, U., Zeitelhofer-Adzemovic, M., Fazekas, F., and Hochmeister, S. (2019). The formation of a glial scar does not prohibit remyelination in an animal model of multiple sclerosis. Glia 67, 467-481.

Halestrap, A.P. (2013). The SLC16 gene family - structure, role and regulation in health and disease. Molecular aspects of medicine $34,337-349$.

Hall, S.M. (1972). The effect of injections of lysophosphatidyl choline into white matter of the adult mouse spinal cord. Journal of cell science 10, 535-546.

Harbo, H.F., Gold, R., and Tintore, M. (2013). Sex and gender issues in multiple sclerosis. Therapeutic advances in neurological disorders 6, 237-248.

Harirchian, M.H., Fatehi, F., Sarraf, P., Honarvar, N.M., and Bitarafan, S. (2018). Worldwide prevalence of familial multiple sclerosis: A systematic review and meta-analysis. Multiple sclerosis and related disorders $20,43-47$. 
Harrison, B. (1985). Schwann cell and oligodendrocyte remyelination in lysolecithin-induced lesions in irradiated rat spinal cord. Journal of the neurological sciences $67,143-159$.

Harsan, L.A., Steibel, J., Zaremba, A., Agin, A., Sapin, R., Poulet, P., Guignard, B., Parizel, N., Grucker, D., Boehm, N., et al. (2008). Recovery from chronic demyelination by thyroid hormone therapy: myelinogenesis induction and assessment by diffusion tensor magnetic resonance imaging. The Journal of neuroscience : the official journal of the Society for Neuroscience 28, 14189-14201.

Hendrickx, D.A.E., van Scheppingen, J., van der Poel, M., Bossers, K., Schuurman, K.G., van Eden, C.G., Hol, E.M., Hamann, J., and Huitinga, I. (2017). Gene Expression Profiling of Multiple Sclerosis Pathology Identifies Early Patterns of Demyelination Surrounding Chronic Active Lesions. Frontiers in immunology 8, 1810.

Herz, J., and Chen, Y. (2006). Reelin, lipoprotein receptors and synaptic plasticity. Nature reviews. Neuroscience 7, 850-859.

Hesse, A., Wagner, M., Held, J., Bruck, W., Salinas-Riester, G., Hao, Z., Waisman, A., and Kuhlmann, T. (2010). In toxic demyelination oligodendroglial cell death occurs early and is FAS independent. Neurobiology of disease 37, 362-369.

Hirsch-Reinshagen, V., Zhou, S., Burgess, B.L., Bernier, L., Mclsaac, S.A., Chan, J.Y., Tansley, G.H., Cohn, J.S., Hayden, M.R., and Wellington, C.L. (2004). Deficiency of ABCA1 impairs apolipoprotein E metabolism in brain. The Journal of biological chemistry 279, 41197-41207.

Hogenboom, S., Romeijn, G.J., Houten, S.M., Baes, M., Wanders, R.J., and Waterham, H.R. (2002). Absence of functional peroxisomes does not lead to deficiency of enzymes involved in cholesterol biosynthesis. Journal of lipid research 43, 90-98.

Hogenboom, S., Tuyp, J.J., Espeel, M., Koster, J., Wanders, R.J., and Waterham, H.R. (2004a). Human mevalonate pyrophosphate decarboxylase is localized in the cytosol. Molecular genetics and metabolism 81, 216-224.

Hogenboom, S., Tuyp, J.J., Espeel, M., Koster, J., Wanders, R.J., and Waterham, H.R. (2004b). Mevalonate kinase is a cytosolic enzyme in humans. Journal of cell science 117, 631-639.

Hooijmans, C.R., Hlavica, M., Schuler, F.A.F., Good, N., Good, A., Baumgartner, L., Galeno, G., Schneider, M.P., Jung, T., de Vries, R., et al. (2019). Remyelination promoting therapies in multiple sclerosis animal models: a systematic review and meta-analysis. Scientific reports 9, 822.

$\mathrm{Hu}, \mathrm{X}$., Li, S., Wu, J., Xia, C., and Lala, D.S. (2003). Liver X receptors interact with corepressors to regulate gene expression. Molecular endocrinology 17, 1019-1026.

Huang, C. (2014). Natural modulators of liver X receptors. Journal of integrative medicine 12, 76-85.

Hughes, T.M., Rosano, C., Evans, R.W., and Kuller, L.H. (2013). Brain cholesterol metabolism, oxysterols, and dementia. Journal of Alzheimer's disease : JAD 33, 891-911.

Hung, Y.H., Bush, A.I., and La Fontaine, S. (2013). Links between copper and cholesterol in Alzheimer's disease. Frontiers in physiology 4, 111.

Hussain, G., Wang, J., Rasul, A., Anwar, H., Imran, A., Qasim, M., Zafar, S., Kamran, S.K.S., Razzaq, A., Aziz, N., et al. (2019). Role of cholesterol and sphingolipids in brain development and neurological diseases. Lipids in health and disease 18, 26.

ladecola, C. (2017). The Neurovascular Unit Coming of Age: A Journey through Neurovascular Coupling in Health and Disease. Neuron 96, 17-42. 
Igarashi, Y., Utsumi, H., Chiba, H., Yamada-Sasamori, Y., Tobioka, H., Kamimura, Y., Furuuchi, K., Kokai, Y., Nakagawa, T., Mori, M., et al. (1999). Glial cell line-derived neurotrophic factor induces barrier function of endothelial cells forming the blood-brain barrier. Biochemical and biophysical research communications $261,108-112$.

Infante, R.E., Wang, M.L., Radhakrishnan, A., Kwon, H.J., Brown, M.S., and Goldstein, J.L. (2008). NPC2 facilitates bidirectional transfer of cholesterol between NPC1 and lipid bilayers, a step in cholesterol egress from lysosomes. Proceedings of the National Academy of Sciences of the United States of America 105, 15287-15292.

International Multiple Sclerosis Genetics, C., Hafler, D.A., Compston, A., Sawcer, S., Lander, E.S., Daly, M.J., De Jager, P.L., de Bakker, P.I., Gabriel, S.B., Mirel, D.B., et al. (2007). Risk alleles for multiple sclerosis identified by a genomewide study. The New England journal of medicine 357, $851-$ 862.

Irvine, K.A., and Blakemore, W.F. (2006). Age increases axon loss associated with primary demyelination in cuprizone-induced demyelination in C57BL/6 mice. Journal of neuroimmunology 175 , 69-76.

Itoh, N., Itoh, Y., Tassoni, A., Ren, E., Kaito, M., Ohno, A., Ao, Y., Farkhondeh, V., Johnsonbaugh, H., Burda, J., et al. (2018). Cell-specific and region-specific transcriptomics in the multiple sclerosis model: Focus on astrocytes. Proceedings of the National Academy of Sciences of the United States of America 115, E302-E309.

Jakel, S., Agirre, E., Mendanha Falcao, A., van Bruggen, D., Lee, K.W., Knuesel, I., Malhotra, D., Ffrench-Constant, C., Williams, A., and Castelo-Branco, G. (2019). Altered human oligodendrocyte heterogeneity in multiple sclerosis. Nature 566, 543-547.

Jankovic, D., and Feng, C.G. (2015). CD4(+) T Cell Differentiation in Infection: Amendments to the Th1/Th2 Axiom. Frontiers in immunology 6, 198.

Jarius, S., Konig, F.B., Metz, I., Ruprecht, K., Paul, F., Bruck, W., and Wildemann, B. (2017). Pattern II and pattern III MS are entities distinct from pattern I MS: evidence from cerebrospinal fluid analysis. Journal of neuroinflammation 14, 171.

Jia, W., Martin, T.A., Zhang, G., and Jiang, W.G. (2013). Junctional adhesion molecules in cerebral endothelial tight junction and brain metastasis. Anticancer research 33, 2353-2359.

Johnson, E.S., and Ludwin, S.K. (1981). The demonstration of recurrent demyelination and remyelination of axons in the central nervous system. Acta neuropathologica 53, 93-98.

Kandutsch, A.A., and Russell, A.E. (1960a). Preputial gland tumor sterols. 2. The identification of 4 alpha-methyl-Delta 8-cholesten-3 beta-ol. The Journal of biological chemistry 235, 2253-2255.

Kandutsch, A.A., and Russell, A.E. (1960b). Preputial gland tumor sterols. 3. A metabolic pathway from lanosterol to cholesterol. The Journal of biological chemistry 235, 2256-2261.

Kanerva, K., Uronen, R.L., Blom, T., Li, S., Bittman, R., Lappalainen, P., Peranen, J., Raposo, G., and Ikonen, E. (2013). LDL cholesterol recycles to the plasma membrane via a Rab8a-Myosin5b-actindependent membrane transport route. Developmental cell 27, 249-262.

Kang, Z., Liu, L., Spangler, R., Spear, C., Wang, C., Gulen, M.F., Veenstra, M., Ouyang, W., Ransohoff, R.M., and Li, X. (2012). IL-17-induced Act1-mediated signaling is critical for cuprizoneinduced demyelination. The Journal of neuroscience : the official journal of the Society for Neuroscience 32, 8284-8292.

Karasinska, J.M., Rinninger, F., Lutjohann, D., Ruddle, P., Franciosi, S., Kruit, J.K., Singaraja, R.R., Hirsch-Reinshagen, V., Fan, J., Brunham, L.R., et al. (2009). Specific Loss of Brain ABCA1 Increases 
Brain Cholesterol Uptake and Influences Neuronal Structure and Function. Journal of Neuroscience 29, 3579-3589.

Keegan, M., Konig, F., McClelland, R., Bruck, W., Morales, Y., Bitsch, A., Panitch, H., Lassmann, H., Weinshenker, B., Rodriguez, M., et al. (2005). Relation between humoral pathological changes in multiple sclerosis and response to therapeutic plasma exchange. Lancet 366, 579-582.

Keirstead, H.S., and Blakemore, W.F. (1997). Identification of post-mitotic oligodendrocytes incapable of remyelination within the demyelinated adult spinal cord. Journal of neuropathology and experimental neurology 56, 1191-1201.

Kermode, A.G., Thompson, A.J., Tofts, P., MacManus, D.G., Kendall, B.E., Kingsley, D.P., Moseley, I.F., Rudge, P., and McDonald, W.I. (1990). Breakdown of the blood-brain barrier precedes symptoms and other MRI signs of new lesions in multiple sclerosis. Pathogenetic and clinical implications. Brain : a journal of neurology 113 ( Pt 5), 1477-1489.

Kipp, M., Clarner, T., Dang, J., Copray, S., and Beyer, C. (2009). The cuprizone animal model: new insights into an old story. Acta neuropathologica 118, 723-736.

Kipp, M., van der Star, B., Vogel, D.Y., Puentes, F., van der Valk, P., Baker, D., and Amor, S. (2012). Experimental in vivo and in vitro models of multiple sclerosis: EAE and beyond. Multiple sclerosis and related disorders 1, 15-28.

Kirk, J., Plumb, J., Mirakhur, M., and McQuaid, S. (2003). Tight junctional abnormality in multiple sclerosis white matter affects all calibres of vessel and is associated with blood-brain barrier leakage and active demyelination. The Journal of pathology 201, 319-327.

Kirk, S.L., and Karlik, S.J. (2003). VEGF and vascular changes in chronic neuroinflammation. Journal of autoimmunity $21,353-363$.

Klicek, R., Kolenc, D., Suran, J., Drmic, D., Brcic, L., Aralica, G., Sever, M., Holjevac, J., Radic, B., Turudic, T., et al. (2013). Stable gastric pentadecapeptide BPC 157 heals cysteamine-colitis and colon-colon-anastomosis and counteracts cuprizone brain injuries and motor disability. Journal of physiology and pharmacology : an official journal of the Polish Physiological Society 64, 597-612.

Koch, S., Donarski, N., Goetze, K., Kreckel, M., Stuerenburg, H.J., Buhmann, C., and Beisiegel, U. (2001). Characterization of four lipoprotein classes in human cerebrospinal fluid. Journal of lipid research 42, 1143-1151.

Kondo, A., Nakano, T., and Suzuki, K. (1987). Blood-brain barrier permeability to horseradish peroxidase in twitcher and cuprizone-intoxicated mice. Brain research 425, 186-190.

Konig, F.B., Wildemann, B., Nessler, S., Zhou, D., Hemmer, B., Metz, I., Hartung, H.P., Kieseier, B.C., and Bruck, W. (2008). Persistence of immunopathological and radiological traits in multiple sclerosis. Archives of neurology 65, 1527-1532.

Kornek, B., Storch, M.K., Weissert, R., Wallstroem, E., Stefferl, A., Olsson, T., Linington, C., Schmidbauer, M., and Lassmann, H. (2000). Multiple sclerosis and chronic autoimmune encephalomyelitis: a comparative quantitative study of axonal injury in active, inactive, and remyelinated lesions. The American journal of pathology 157, 267-276.

Kovacs, W.J., Tape, K.N., Shackelford, J.E., Duan, X., Kasumov, T., Kelleher, J.K., Brunengraber, H., and Krisans, S.K. (2007). Localization of the pre-squalene segment of the isoprenoid biosynthetic pathway in mammalian peroxisomes. Histochemistry and cell biology 127, 273-290.

Krishnamoorthy, G., Lassmann, H., Wekerle, H., and Holz, A. (2006). Spontaneous opticospinal encephalomyelitis in a double-transgenic mouse model of autoimmune T cell/B cell cooperation. The Journal of clinical investigation 116, 2385-2392. 
Kuhlmann, T., Miron, V., Cui, Q., Wegner, C., Antel, J., and Bruck, W. (2008). Differentiation block of oligodendroglial progenitor cells as a cause for remyelination failure in chronic multiple sclerosis. Brain : a journal of neurology 131, 1749-1758.

Laman, J.D., Thompson, E.J., and Kappos, L. (1998). Balancing the Th1/Th2 concept in multiple sclerosis. Immunology today 19, 489-490.

Lane-Donovan, C., Philips, G.T., and Herz, J. (2014). More than cholesterol transporters: lipoprotein receptors in CNS function and neurodegeneration. Neuron 83, 771-787.

Lassmann, H. (2013). Pathology and disease mechanisms in different stages of multiple sclerosis. Journal of the neurological sciences $333,1-4$.

Lassmann, H. (2017). Targets of therapy in progressive MS. Multiple sclerosis 23, 1593-1599.

Lassmann, H. (2018). Pathogenic Mechanisms Associated With Different Clinical Courses of Multiple Sclerosis. Frontiers in immunology 9, 3116.

Lassmann, H., and Bradl, M. (2017). Multiple sclerosis: experimental models and reality. Acta neuropathologica 133, 223-244.

Lavrnja, I., Smiljanic, K., Savic, D., Mladenovic-Djordjevic, A., Tesovic, K., Kanazir, S., and Pekovic, S. (2017). Expression profiles of cholesterol metabolism-related genes are altered during development of experimental autoimmune encephalomyelitis in the rat spinal cord. Scientific reports 7, 2702.

Lewandowsky, M. (1900). Zur Lehre von der Cerebrospinalflüssigkeit. Z. Klin. Med. 40, 480-494.

$\mathrm{Li}, \mathrm{H}$. (2019). Cholesterol esters of oligodendrocytes in developmental and ageing brain.

(Biotechnology and Biological Sciences Research Council (BBSRC)).

Li, L., Zhang, H., Varrin-Doyer, M., Zamvil, S.S., and Verkman, A.S. (2011). Proinflammatory role of aquaporin-4 in autoimmune neuroinflammation. FASEB journal : official publication of the Federation of American Societies for Experimental Biology 25, 1556-1566.

Li, W., Chen, Z., Chin, I., Chen, Z., and Dai, H. (2018). The Role of VE-cadherin in Blood-brain Barrier Integrity Under Central Nervous System Pathological Conditions. Current neuropharmacology 16, $1375-1384$.

Linton, M.F., Gish, R., Hubl, S.T., Butler, E., Esquivel, C., Bry, W.I., Boyles, J.K., Wardell, M.R., and Young, S.G. (1991). Phenotypes of apolipoprotein B and apolipoprotein E after liver transplantation. The Journal of clinical investigation 88, 270-281.

Liscum, L. (2002). Cholesterol Biosynthesis. (Elsevier Science B.V.).

Litzenburger, T., Fassler, R., Bauer, J., Lassmann, H., Linington, C., Wekerle, H., and Iglesias, A. (1998). B lymphocytes producing demyelinating autoantibodies: development and function in genetargeted transgenic mice. The Journal of experimental medicine 188, 169-180.

Lodygin, D., Hermann, M., Schweingruber, N., Flugel-Koch, C., Watanabe, T., Schlosser, C., Merlini, A., Korner, H., Chang, H.F., Fischer, H.J., et al. (2019). Publisher Correction: beta-Synuclein-reactive T cells induce autoimmune CNS grey matter degeneration. Nature 567, E15.

Lodygin, D., Odoardi, F., Schlager, C., Korner, H., Kitz, A., Nosov, M., van den Brandt, J., Reichardt, H.M., Haberl, M., and Flugel, A. (2013). A combination of fluorescent NFAT and H2B sensors uncovers dynamics of T cell activation in real time during CNS autoimmunity. Nature medicine 19, 784-790. 
Loeffler, B., Heeren, J., Blaeser, M., Radner, H., Kayser, D., Aydin, B., and Merkel, M. (2007). Lipoprotein lipase-facilitated uptake of LDL is mediated by the LDL receptor. Journal of lipid research 48, 288-298.

Lorscheider, J., Buzzard, K., Jokubaitis, V., Spelman, T., Havrdova, E., Horakova, D., Trojano, M., Izquierdo, G., Girard, M., Duquette, P., et al. (2016). Defining secondary progressive multiple sclerosis. Brain : a journal of neurology 139, 2395-2405.

Loscher, W., and Potschka, H. (2005). Blood-brain barrier active efflux transporters: ATP-binding cassette gene family. NeuroRx : the journal of the American Society for Experimental NeuroTherapeutics 2, 86-98.

Lucchinetti, C., Bruck, W., Parisi, J., Scheithauer, B., Rodriguez, M., and Lassmann, H. (1999). A quantitative analysis of oligodendrocytes in multiple sclerosis lesions. A study of 113 cases. Brain : a journal of neurology 122 (Pt 12), 2279-2295.

Lucchinetti, C., Bruck, W., Parisi, J., Scheithauer, B., Rodriguez, M., and Lassmann, H. (2000). Heterogeneity of multiple sclerosis lesions: implications for the pathogenesis of demyelination. Annals of neurology $47,707-717$.

Lucchinetti, C.F., Gavrilova, R.H., Metz, I., Parisi, J.E., Scheithauer, B.W., Weigand, S., Thomsen, K., Mandrekar, J., Altintas, A., Erickson, B.J., et al. (2008). Clinical and radiographic spectrum of pathologically confirmed tumefactive multiple sclerosis. Brain : a journal of neurology 131, 1759-1775.

Luhder, F., Kebir, H., Odoardi, F., Litke, T., Sonneck, M., Alvarez, J.I., Winchenbach, J., Eckert, N., Hayardeny, L., Sorani, E., et al. (2017). Laquinimod enhances central nervous system barrier functions. Neurobiology of disease 102, 60-69.

Luissint, A.C., Artus, C., Glacial, F., Ganeshamoorthy, K., and Couraud, P.O. (2012). Tight junctions at the blood brain barrier: physiological architecture and disease-associated dysregulation. Fluids and barriers of the CNS 9, 23.

Lund, E.G., Guileyardo, J.M., and Russell, D.W. (1999). cDNA cloning of cholesterol 24-hydroxylase, a mediator of cholesterol homeostasis in the brain. Proceedings of the National Academy of Sciences of the United States of America 96, 7238-7243.

Luo, J., Ho, P., Steinman, L., and Wyss-Coray, T. (2008). Bioluminescence in vivo imaging of autoimmune encephalomyelitis predicts disease. Journal of neuroinflammation 5, 6 .

Luo, J., Yang, H., and Song, B.-L. (2019). Mechanisms and regulation of cholesterol homeostasis. Nature Reviews Molecular Cell Biology.

Luu, W., Hart-Smith, G., Sharpe, L.J., and Brown, A.J. (2015). The terminal enzymes of cholesterol synthesis, DHCR24 and DHCR7, interact physically and functionally. Journal of lipid research 56, 888897.

Mahad, D., Lassmann, H., and Turnbull, D. (2008). Review: Mitochondria and disease progression in multiple sclerosis. Neuropathology and applied neurobiology 34, 577-589.

Mahad, D.J., and Ransohoff, R.M. (2003). The role of MCP-1 (CCL2) and CCR2 in multiple sclerosis and experimental autoimmune encephalomyelitis (EAE). Seminars in immunology 15, 23-32.

Mahley, R.W. (2016). Central Nervous System Lipoproteins: ApoE and Regulation of Cholesterol Metabolism. Arteriosclerosis, thrombosis, and vascular biology 36, 1305-1315.

Malik, V.A., and Di Benedetto, B. (2018). The Blood-Brain Barrier and the EphR/Ephrin System: Perspectives on a Link Between Neurovascular and Neuropsychiatric Disorders. Frontiers in molecular neuroscience 11, 127. 
Manrique-Hoyos, N., Jurgens, T., Gronborg, M., Kreutzfeldt, M., Schedensack, M., Kuhlmann, T., Schrick, C., Bruck, W., Urlaub, H., Simons, M., et al. (2012). Late motor decline after accomplished remyelination: impact for progressive multiple sclerosis. Annals of neurology 71, 227-244.

Maoz, B.M., Herland, A., FitzGerald, E.A., Grevesse, T., Vidoudez, C., Pacheco, A.R., Sheehy, S.P., Park, T.E., Dauth, S., Mannix, R., et al. (2018). A linked organ-on-chip model of the human neurovascular unit reveals the metabolic coupling of endothelial and neuronal cells. Nature biotechnology $36,865-874$.

Marchiando, A.M., Shen, L., Graham, W.V., Weber, C.R., Schwarz, B.T., Austin, J.R., 2nd, Raleigh, D.R., Guan, Y., Watson, A.J., Montrose, M.H., et al. (2010). Caveolin-1-dependent occludin endocytosis is required for TNF-induced tight junction regulation in vivo. The Journal of cell biology $189,111-126$.

Marques, S., Zeisel, A., Codeluppi, S., van Bruggen, D., Mendanha Falcao, A., Xiao, L., Li, H., Haring, M., Hochgerner, H., Romanov, R.A., et al. (2016). Oligodendrocyte heterogeneity in the mouse juvenile and adult central nervous system. Science 352, 1326-1329.

Martin, M.G., Pfrieger, F., and Dotti, C.G. (2014). Cholesterol in brain disease: sometimes determinant and frequently implicated. EMBO reports $15,1036-1052$.

Mason, J.L., Toews, A., Hostettler, J.D., Morell, P., Suzuki, K., Goldman, J.E., and Matsushima, G.K. (2004). Oligodendrocytes and progenitors become progressively depleted within chronically demyelinated lesions. The American journal of pathology 164, 1673-1682.

Matsushima, G.K., and Morell, P. (2001). The neurotoxicant, cuprizone, as a model to study demyelination and remyelination in the central nervous system. Brain pathology 11, 107-116.

Mauch, D.H., Nagler, K., Schumacher, S., Goritz, C., Muller, E.C., Otto, A., and Pfrieger, F.W. (2001). CNS synaptogenesis promoted by glia-derived cholesterol. Science 294, 1354-1357.

Mazein, A., Watterson, S., Hsieh, W.Y., Griffiths, W.J., and Ghazal, P. (2013). A comprehensive machine-readable view of the mammalian cholesterol biosynthesis pathway. Biochemical pharmacology $86,56-66$.

McMahon, E.J., Suzuki, K., and Matsushima, G.K. (2002). Peripheral macrophage recruitment in cuprizone-induced CNS demyelination despite an intact blood-brain barrier. Journal of neuroimmunology $130,32-45$.

Metz, I., Weigand, S.D., Popescu, B.F., Frischer, J.M., Parisi, J.E., Guo, Y., Lassmann, H., Bruck, W., and Lucchinetti, C.F. (2014). Pathologic heterogeneity persists in early active multiple sclerosis lesions. Annals of neurology 75, 728-738.

Miller, D.H., Chard, D.T., and Ciccarelli, O. (2012). Clinically isolated syndromes. The Lancet. Neurology 11, 157-169.

Miron, V.E., Zehntner, S.P., Kuhlmann, T., Ludwin, S.K., Owens, T., Kennedy, T.E., Bedell, B.J., and Antel, J.P. (2009). Statin therapy inhibits remyelination in the central nervous system. The American journal of pathology $174,1880-1890$.

Mitsche, M.A., McDonald, J.G., Hobbs, H.H., and Cohen, J.C. (2015). Flux analysis of cholesterol biosynthesis in vivo reveals multiple tissue and cell-type specific pathways. eLife 4, e07999.

Morcos, Y., Hosie, M.J., Bauer, H.C., and Chan-Ling, T. (2001). Immunolocalization of occludin and claudin-1 to tight junctions in intact CNS vessels of mammalian retina. Journal of neurocytology 30 , 107-123.

Morita, K., Sasaki, H., Furuse, M., and Tsukita, S. (1999). Endothelial claudin: claudin-5/TMVCF constitutes tight junction strands in endothelial cells. The Journal of cell biology 147, 185-194. 
Moutsianas, L., Jostins, L., Beecham, A.H., Dilthey, A.T., Xifara, D.K., Ban, M., Shah, T.S., Patsopoulos, N.A., Alfredsson, L., Anderson, C.A., et al. (2015). Class II HLA interactions modulate genetic risk for multiple sclerosis. Nature genetics $47,1107-1113$.

Mundt, S., Greter, M., Flugel, A., and Becher, B. (2019). The CNS Immune Landscape from the Viewpoint of a T Cell. Trends in neurosciences 42, 667-679.

Munji, R.N., Soung, A.L., Weiner, G.A., Sohet, F., Semple, B.D., Trivedi, A., Gimlin, K., Kotoda, M., Korai, M., Aydin, S., et al. (2019). Profiling the mouse brain endothelial transcriptome in health and disease models reveals a core blood-brain barrier dysfunction module. Nature neuroscience 22, 18921902.

Muoio, V., Persson, P.B., and Sendeski, M.M. (2014). The neurovascular unit - concept review. Acta physiologica 210, 790-798.

Muse, E.D., Yu, S., Edillor, C.R., Tao, J., Spann, N.J., Troutman, T.D., Seidman, J.S., Henke, A., Roland, J.T., Ozeki, K.A., et al. (2018). Cell-specific discrimination of desmosterol and desmosterol mimetics confers selective regulation of LXR and SREBP in macrophages. Proceedings of the National Academy of Sciences of the United States of America 115, E4680-E4689.

Nag, S., and Begley, D. (2005). Blood-brain barrier, exchange of metabolites and gases. ISN Neuropath. Press Pathology and Genetics, 22-29.

Nag, S., Manias, J.L., and Stewart, D.J. (2009). Pathology and new players in the pathogenesis of brain edema. Acta neuropathologica 118, 197-217.

Nakahara, J. (2017). Remyelination in multiple sclerosis: Pathology and treatment strategies. Clinical and Experimental Neuroimmunology Volume 8, 40-46.

Nakanishi, M., Goldstein, J.L., and Brown, M.S. (1988). Multivalent control of 3-hydroxy-3methylglutaryl coenzyme A reductase. Mevalonate-derived product inhibits translation of mRNA and accelerates degradation of enzyme. The Journal of biological chemistry 263, 8929-8937.

Nieweg, K., Schaller, H., and Pfrieger, F.W. (2009). Marked differences in cholesterol synthesis between neurons and glial cells from postnatal rats. Journal of neurochemistry 109, 125-134.

Nitta, T., Hata, M., Gotoh, S., Seo, Y., Sasaki, H., Hashimoto, N., Furuse, M., and Tsukita, S. (2003). Size-selective loosening of the blood-brain barrier in claudin-5-deficient mice. The Journal of cell biology 161, 653-660.

O'Gorman, C., Lucas, R., and Taylor, B. (2012). Environmental risk factors for multiple sclerosis: a review with a focus on molecular mechanisms. International journal of molecular sciences $13,11718-$ 11752.

Olah, M., Amor, S., Brouwer, N., Vinet, J., Eggen, B., Biber, K., and Boddeke, H.W. (2012). Identification of a microglia phenotype supportive of remyelination. Glia 60, 306-321.

Oldendorf, W.H., Cornford, M.E., and Brown, W.J. (1977). The large apparent work capability of the blood-brain barrier: a study of the mitochondrial content of capillary endothelial cells in brain and other tissues of the rat. Annals of neurology 1, 409-417.

Olivier, L.M., and Krisans, S.K. (2000). Peroxisomal protein targeting and identification of peroxisomal targeting signals in cholesterol biosynthetic enzymes. Biochimica et biophysica acta 1529, 89-102.

Olzmann, J.A., and Carvalho, P. (2019). Dynamics and functions of lipid droplets. Nature reviews. Molecular cell biology 20, 137-155. 
Omidi, Y., and Barar, J. (2012). Impacts of blood-brain barrier in drug delivery and targeting of brain tumors. Biolmpacts : BI 2, 5-22.

Oreja-Guevara, C., Ramos-Cejudo, J., Aroeira, L.S., Chamorro, B., and Diez-Tejedor, E. (2012). $\mathrm{TH} 1 / \mathrm{TH} 2$ Cytokine profile in relapsing-remitting multiple sclerosis patients treated with Glatiramer acetate or Natalizumab. BMC neurology 12, 95.

Orth, M., and Bellosta, S. (2012). Cholesterol: its regulation and role in central nervous system disorders. Cholesterol 2012, 292598.

Ortiz, J.A., Gil-Gomez, G., Casaroli-Marano, R.P., Vilaro, S., Hegardt, F.G., and Haro, D. (1994). Transfection of the ketogenic mitochondrial 3-hydroxy-3-methylglutaryl-coenzyme A synthase cDNA into Mev- 1 cells corrects their auxotrophy for mevalonate. The Journal of biological chemistry 269, 28523-28526.

Park, J., Matralis, A.N., Berghuis, A.M., and Tsantrizos, Y.S. (2014). Human isoprenoid synthase enzymes as therapeutic targets. Frontiers in chemistry 2, 50.

Parnell, G.P., and Booth, D.R. (2017). The Multiple Sclerosis (MS) Genetic Risk Factors Indicate both Acquired and Innate Immune Cell Subsets Contribute to MS Pathogenesis and Identify Novel Therapeutic Opportunities. Frontiers in immunology 8, 425.

Patrikios, P., Stadelmann, C., Kutzelnigg, A., Rauschka, H., Schmidbauer, M., Laursen, H., Sorensen, P.S., Bruck, W., Lucchinetti, C., and Lassmann, H. (2006). Remyelination is extensive in a subset of multiple sclerosis patients. Brain : a journal of neurology 129, 3165-3172.

Paul, D., Ge, S., Lemire, Y., Jellison, E.R., Serwanski, D.R., Ruddle, N.H., and Pachter, J.S. (2014). Cell-selective knockout and 3D confocal image analysis reveals separate roles for astrocyte-and endothelial-derived CCL2 in neuroinflammation. Journal of neuroinflammation 11, 10.

Paul, R., Koedel, U., Winkler, F., Kieseier, B.C., Fontana, A., Kopf, M., Hartung, H.P., and Pfister, H.W. (2003). Lack of IL-6 augments inflammatory response but decreases vascular permeability in bacterial meningitis. Brain : a journal of neurology 126, 1873-1882.

Penderis, J., Shields, S.A., and Franklin, R.J. (2003). Impaired remyelination and depletion of oligodendrocyte progenitors does not occur following repeated episodes of focal demyelination in the rat central nervous system. Brain : a journal of neurology 126, 1382-1391.

Peters, A., Palay, S.L., and Webster, H. (1991). The Fine Structure of the Nervous System: Neurons and Their Supporting Cells. (Oxford University Press,).

Petrov, A.M., Kasimov, M.R., and Zefirov, A.L. (2016). Brain Cholesterol Metabolism and Its Defects: Linkage to Neurodegenerative Diseases and Synaptic Dysfunction. Acta naturae 8, 58-73.

Petzold, G.C., and Murthy, V.N. (2011). Role of astrocytes in neurovascular coupling. Neuron 71, 782797.

Pfeffer, S.R. (2019). NPC intracellular cholesterol transporter 1 (NPC1)-mediated cholesterol export from lysosomes. The Journal of biological chemistry 294, 1706-1709.

Pfeiffer, F., Schafer, J., Lyck, R., Makrides, V., Brunner, S., Schaeren-Wiemers, N., Deutsch, U., and Engelhardt, B. (2011). Claudin-1 induced sealing of blood-brain barrier tight junctions ameliorates chronic experimental autoimmune encephalomyelitis. Acta neuropathologica 122, 601-614.

Pfrieger, F.W., and Ungerer, N. (2011). Cholesterol metabolism in neurons and astrocytes. Progress in lipid research 50, 357-371. 
Pinholt, M., Frederiksen, J.L., and Christiansen, M. (2006). The association between apolipoprotein E and multiple sclerosis. European journal of neurology 13, 573-580.

Pittock, S.J., McClelland, R.L., Achenbach, S.J., Konig, F., Bitsch, A., Bruck, W., Lassmann, H., Parisi, J.E., Scheithauer, B.W., Rodriguez, M., et al. (2005). Clinical course, pathological correlations, and outcome of biopsy proved inflammatory demyelinating disease. Journal of neurology, neurosurgery, and psychiatry 76, 1693-1697.

Pollinger, B., Krishnamoorthy, G., Berer, K., Lassmann, H., Bosl, M.R., Dunn, R., Domingues, H.S., Holz, A., Kurschus, F.C., and Wekerle, H. (2009). Spontaneous relapsing-remitting EAE in the SJL/J mouse: MOG-reactive transgenic T cells recruit endogenous MOG-specific B cells. The Journal of experimental medicine 206, 1303-1316.

Popescu, B.F., Pirko, I., and Lucchinetti, C.F. (2013). Pathology of multiple sclerosis: where do we stand? Continuum 19, 901-921.

Praet, J., Guglielmetti, C., Berneman, Z., Van der Linden, A., and Ponsaerts, P. (2014). Cellular and molecular neuropathology of the cuprizone mouse model: clinical relevance for multiple sclerosis. Neuroscience and biobehavioral reviews 47, 485-505.

Prineas, J.W., Barnard, R.O., Kwon, E.E., Sharer, L.R., and Cho, E.S. (1993a). Multiple sclerosis: remyelination of nascent lesions. Annals of neurology 33, 137-151.

Prineas, J.W., Barnard, R.O., Revesz, T., Kwon, E.E., Sharer, L., and Cho, E.S. (1993b). Multiple sclerosis. Pathology of recurrent lesions. Brain : a journal of neurology 116 ( Pt 3), 681-693.

Prineas, J.W., Kwon, E.E., Goldenberg, P.Z., llyas, A.A., Quarles, R.H., Benjamins, J.A., and Sprinkle, T.J. (1989). Multiple sclerosis. Oligodendrocyte proliferation and differentiation in fresh lesions. Laboratory investigation; a journal of technical methods and pathology 61, 489-503.

Procaccini, C., De Rosa, V., Pucino, V., Formisano, L., and Matarese, G. (2015). Animal models of Multiple Sclerosis. European journal of pharmacology 759, 182-191.

Quintana, F.J., Farez, M.F., Viglietta, V., Iglesias, A.H., Merbl, Y., Izquierdo, G., Lucas, M., Basso, A.S., Khoury, S.J., Lucchinetti, C.F., et al. (2008). Antigen microarrays identify unique serum autoantibody signatures in clinical and pathologic subtypes of multiple sclerosis. Proceedings of the National Academy of Sciences of the United States of America 105, 18889-18894.

Rabchevsky, A.G., Degos, J.D., and Dreyfus, P.A. (1999). Peripheral injections of Freund's adjuvant in mice provoke leakage of serum proteins through the blood-brain barrier without inducing reactive gliosis. Brain research 832, 84-96.

Radhakrishnan, A., Goldstein, J.L., McDonald, J.G., and Brown, M.S. (2008). Switch-like control of SREBP-2 transport triggered by small changes in ER cholesterol: a delicate balance. Cell metabolism $8,512-521$.

Raine, C.S., and Wu, E. (1993). Multiple sclerosis: remyelination in acute lesions. Journal of neuropathology and experimental neurology 52, 199-204.

Ramagopalan, S.V., Dyment, D.A., Cader, M.Z., Morrison, K.M., Disanto, G., Morahan, J.M., Berlanga-Taylor, A.J., Handel, A., De Luca, G.C., Sadovnick, A.D., et al. (2011). Rare variants in the CYP27B1 gene are associated with multiple sclerosis. Annals of neurology 70, 881-886.

Ramirez, D.M., Andersson, S., and Russell, D.W. (2008). Neuronal expression and subcellular localization of cholesterol 24-hydroxylase in the mouse brain. The Journal of comparative neurology 507, 1676-1693.

Ramirez, O.A., and Couve, A. (2011). The endoplasmic reticulum and protein trafficking in dendrites and axons. Trends Cell Biol 21, 219-227. 
Ransohoff, R.M. (2012). Animal models of multiple sclerosis: the good, the bad and the bottom line. Nature neuroscience 15, 1074-1077.

Recks, M.S., Addicks, K., and Kuerten, S. (2011). Spinal cord histopathology of MOG peptide 35-55induced experimental autoimmune encephalomyelitis is time- and score-dependent. Neuroscience letters 494, 227-231.

Reese, T.S., and Karnovsky, M.J. (1967). Fine structural localization of a blood-brain barrier to exogenous peroxidase. The Journal of cell biology 34, 207-217.

Reiber, H., Suckling, A.J., and Rumsby, M.G. (1984). The effect of Freund's adjuvants on bloodcerebrospinal fluid barrier permeability. Journal of the neurological sciences $63,55-61$.

Reuss, B., Dono, R., and Unsicker, K. (2003). Functions of fibroblast growth factor (FGF)-2 and FGF-5 in astroglial differentiation and blood-brain barrier permeability: evidence from mouse mutants. The Journal of neuroscience : the official journal of the Society for Neuroscience 23, 6404-6412.

Richard, J.F., Roy, M., Audoy-Remus, J., Tremblay, P., and Vallieres, L. (2011). Crawling phagocytes recruited in the brain vasculature after pertussis toxin exposure through IL6, ICAM1 and ITGalphaM. Brain pathology 21, 661-671.

Robinson, A.P., Harp, C.T., Noronha, A., and Miller, S.D. (2014). The experimental autoimmune encephalomyelitis (EAE) model of MS: utility for understanding disease pathophysiology and treatment. Handbook of clinical neurology 122, 173-189.

Rochfort, K.D., Collins, L.E., Murphy, R.P., and Cummins, P.M. (2014). Downregulation of blood-brain barrier phenotype by proinflammatory cytokines involves NADPH oxidase-dependent ROS generation: consequences for interendothelial adherens and tight junctions. PloS one 9, e101815.

Rodgers, J.M., Robinson, A.P., and Miller, S.D. (2013). Strategies for protecting oligodendrocytes and enhancing remyelination in multiple sclerosis. Discovery medicine 16, 53-63.

Roemer, S.F., Parisi, J.E., Lennon, V.A., Benarroch, E.E., Lassmann, H., Bruck, W., Mandler, R.N., Weinshenker, B.G., Pittock, S.J., Wingerchuk, D.M., et al. (2007). Pattern-specific loss of aquaporin-4 immunoreactivity distinguishes neuromyelitis optica from multiple sclerosis. Brain : a journal of neurology 130, 1194-1205.

Rosenberg, G.A. (2012). Neurological diseases in relation to the blood-brain barrier. Journal of cerebral blood flow and metabolism : official journal of the International Society of Cerebral Blood Flow and Metabolism 32, 1139-1151.

Rosenstein, J.M., Krum, J.M., and Ruhrberg, C. (2010). VEGF in the nervous system. Organogenesis $6,107-114$.

Ross, J.P., Bernales, C.Q., Lee, J.D., Sadovnick, A.D., Traboulsee, A.L., and Vilarino-Guell, C. (2014). Analysis of CYP27B1 in multiple sclerosis. Journal of neuroimmunology 266, 64-66.

Rossi, B., and Constantin, G. (2016). Live Imaging of Immune Responses in Experimental Models of Multiple Sclerosis. Frontiers in immunology 7, 506.

Ruther, B.J., Scheld, M., Dreymueller, D., Clarner, T., Kress, E., Brandenburg, L.O., Swartenbroekx, T., Hoornaert, C., Ponsaerts, P., Fallier-Becker, P., et al. (2017). Combination of cuprizone and experimental autoimmune encephalomyelitis to study inflammatory brain lesion formation and progression. Glia 65, 1900-1913.

Sa-Pereira, I., Brites, D., and Brito, M.A. (2012). Neurovascular unit: a focus on pericytes. Molecular neurobiology 45, 327-347. 
Saeed, A.A., Genove, G., Li, T., Lutjohann, D., Olin, M., Mast, N., Pikuleva, I.A., Crick, P., Wang, Y., Griffiths, W., et al. (2014). Effects of a disrupted blood-brain barrier on cholesterol homeostasis in the brain. The Journal of biological chemistry 289, 23712-23722.

Saharinen, P., Eklund, L., and Alitalo, K. (2017). Therapeutic targeting of the angiopoietin-TIE pathway. Nature reviews. Drug discovery 16, 635-661.

Saher, G., Brugger, B., Lappe-Siefke, C., Mobius, W., Tozawa, R., Wehr, M.C., Wieland, F., Ishibashi, S., and Nave, K.A. (2005). High cholesterol level is essential for myelin membrane growth. Nature neuroscience $8,468-475$.

Saher, G., Quintes, S., and Nave, K.A. (2011). Cholesterol: a novel regulatory role in myelin formation. The Neuroscientist : a review journal bringing neurobiology, neurology and psychiatry 17, 79-93.

Saher, G., Rudolphi, F., Corthals, K., Ruhwedel, T., Schmidt, K.F., Lowel, S., Dibaj, P., Barrette, B., Mobius, W., and Nave, K.A. (2012). Therapy of Pelizaeus-Merzbacher disease in mice by feeding a cholesterol-enriched diet. Nature medicine 18, 1130-1135.

Saher, G., and Stumpf, S.K. (2015). Cholesterol in myelin biogenesis and hypomyelinating disorders. Biochimica et biophysica acta 1851, 1083-1094.

Saito, K., Dubreuil, V., Arai, Y., Wilsch-Brauninger, M., Schwudke, D., Saher, G., Miyata, T., Breier, G., Thiele, C., Shevchenko, A., et al. (2009). Ablation of cholesterol biosynthesis in neural stem cells increases their VEGF expression and angiogenesis but causes neuron apoptosis. Proceedings of the National Academy of Sciences of the United States of America 106, 8350-8355.

Sakai, K., Igarashi, M., Yamamuro, D., Ohshiro, T., Nagashima, S., Takahashi, M., Enkhtuvshin, B., Sekiya, M., Okazaki, H., Osuga, J., et al. (2014). Critical role of neutral cholesteryl ester hydrolase 1 in cholesteryl ester hydrolysis in murine macrophages. Journal of lipid research 55, 2033-2040.

Sawcer, S., Franklin, R.J., and Ban, M. (2014). Multiple sclerosis genetics. The Lancet. Neurology 13, 700-709.

Scheld, M., Ruther, B.J., Grosse-Veldmann, R., Ohl, K., Tenbrock, K., Dreymuller, D., Fallier-Becker, P., Zendedel, A., Beyer, C., Clarner, T., et al. (2016). Neurodegeneration Triggers Peripheral Immune Cell Recruitment into the Forebrain. The Journal of neuroscience : the official journal of the Society for Neuroscience 36, 1410-1415.

Schellenberg, A.E., Buist, R., Yong, V.W., Del Bigio, M.R., and Peeling, J. (2007). Magnetic resonance imaging of blood-spinal cord barrier disruption in mice with experimental autoimmune encephalomyelitis. Magnetic resonance in medicine 58, 298-305.

Schoenheimer, R., and Breusch, F. (1933). Synthesis and destruction of cholesterol. The Journal of biological chemistry 103, 439-448. .

Schuster, G.U., Parini, P., Wang, L., Alberti, S., Steffensen, K.R., Hansson, G.K., Angelin, B., and Gustafsson, J.A. (2002). Accumulation of foam cells in liver X receptor-deficient mice. Circulation 106, 1147-1153.

Scurfield, A., and Latimer, D.C. (2018). A computational study of the impact of inhomogeneous internodal lengths on conduction velocity in myelinated neurons. PloS one 13, e0191106.

Segatto, M., Tonini, C., Pfrieger, F.W., Trezza, V., and Pallottini, V. (2019). Loss of Mevalonate/Cholesterol Homeostasis in the Brain: A Focus on Autism Spectrum Disorder and Rett Syndrome. International journal of molecular sciences 20.

Segrest, J.P., Jones, M.K., De Loof, H., and Dashti, N. (2001). Structure of apolipoprotein B-100 in low density lipoproteins. Journal of lipid research 42, 1346-1367. 
Sharpe, L.J., and Brown, A.J. (2013). Controlling cholesterol synthesis beyond 3-hydroxy-3methylglutaryl-CoA reductase (HMGCR). The Journal of biological chemistry 288, 18707-18715.

Shen, S., Sandoval, J., Swiss, V.A., Li, J., Dupree, J., Franklin, R.J., and Casaccia-Bonnefil, P. (2008). Age-dependent epigenetic control of differentiation inhibitors is critical for remyelination efficiency. Nature neuroscience 11, 1024-1034.

Shibata, N., and Glass, C.K. (2009). Regulation of macrophage function in inflammation and atherosclerosis. Journal of lipid research 50 Suppl, S277-281.

Shigemoto-Mogami, Y., Hoshikawa, K., and Sato, K. (2018). Activated Microglia Disrupt the BloodBrain Barrier and Induce Chemokines and Cytokines in a Rat in vitro Model. Frontiers in cellular neuroscience 12, 494.

Sim, F.J., Zhao, C., Penderis, J., and Franklin, R.J. (2002). The age-related decrease in CNS remyelination efficiency is attributable to an impairment of both oligodendrocyte progenitor recruitment and differentiation. The Journal of neuroscience : the official journal of the Society for Neuroscience 22, 2451-2459.

Simmons, R.D., Bernard, C.C.A., and Carnegie, P.R. (1983). Factors Underlying Ascending Paralysis in Rodents During Experimental Autoimmune Encephalomyelitis (EAE). (Humana Press).

Smets, I., Goris, A., Moisse, M., Vandebergh, M., Lagou, V., Demeestere, J., Mallants, K., Hilven, K., Van Damme, P., Sunaert, S., et al. (2018). Chr8p23 region implicated in genetic basis for demyelination in multiple sclerosis patients. In 34th Congress of the European-Committee-forTreatment-and-Research-in-Multiple-Sclerosis (ECTRIMS) (Berlin: ECTRIMS Online Library).

Smith, K.J., Blakemore, W.F., and McDonald, W.I. (1979). Central remyelination restores secure conduction. Nature 280, 395-396.

Smith, K.J., Blakemore, W.F., and McDonald, W.I. (1981). The restoration of conduction by central remyelination. Brain : a journal of neurology 104, 383-404.

Spann, N.J., Garmire, L.X., McDonald, J.G., Myers, D.S., Milne, S.B., Shibata, N., Reichart, D., Fox, J.N., Shaked, I., Heudobler, D., et al. (2012). Regulated accumulation of desmosterol integrates macrophage lipid metabolism and inflammatory responses. Cell 151, 138-152.

Sparrow, C.P., Baffic, J., Lam, M.H., Lund, E.G., Adams, A.D., Fu, X., Hayes, N., Jones, A.B., Macnaul, K.L., Ondeyka, J., et al. (2002). A potent synthetic LXR agonist is more effective than cholesterol loading at inducing ABCA1 mRNA and stimulating cholesterol efflux. The Journal of biological chemistry 277, 10021-10027.

Stadelmann, C., Wegner, C., and Bruck, W. (2011). Inflammation, demyelination, and degeneration recent insights from MS pathology. Biochimica et biophysica acta 1812, 275-282.

Stamatovic, S.M., Keep, R.F., and Andjelkovic, A.V. (2008). Brain endothelial cell-cell junctions: how to "open" the blood brain barrier. Current neuropharmacology 6, 179-192.

Stamatovic, S.M., Keep, R.F., Kunkel, S.L., and Andjelkovic, A.V. (2003). Potential role of MCP-1 in endothelial cell tight junction 'opening': signaling via Rho and Rho kinase. Journal of cell science 116, 4615-4628.

Steelman, A.J., Thompson, J.P., and Li, J. (2012). Demyelination and remyelination in anatomically distinct regions of the corpus callosum following cuprizone intoxication. Neuroscience research 72, 3242.

Stefani, M., and Liguri, G. (2009). Cholesterol in Alzheimer's disease: unresolved questions. Current Alzheimer research 6, 15-29. 
Stein, E.A., Bays, H., O'Brien, D., Pedicano, J., Piper, E., and Spezzi, A. (2011). Lapaquistat acetate: development of a squalene synthase inhibitor for the treatment of hypercholesterolemia. Circulation 123, 1974-1985.

Stern, L., and Gautier, R. (1921). Recherches Sur Le Liquide CÉphalo-Rachidien: I.-Les Rapports Entre Le Liquide CÉphalo- Rachidien et la Circulation Sanguine. Archives Internationales de Physiologie 17:2, 138-192.

Stidworthy, M.F., Genoud, S., Suter, U., Mantei, N., and Franklin, R.J. (2003). Quantifying the early stages of remyelination following cuprizone-induced demyelination. Brain pathology 13, 329-339.

Stokum, J.A., Gerzanich, V., and Simard, J.M. (2016). Molecular pathophysiology of cerebral edema. Journal of cerebral blood flow and metabolism : official journal of the International Society of Cerebral Blood Flow and Metabolism 36, 513-538.

Strickland, D.K., Gonias, S.L., and Argraves, W.S. (2002). Diverse roles for the LDL receptor family. Trends in endocrinology and metabolism: TEM 13, 66-74.

Subramanian, K., and Balch, W.E. (2008). NPC1/NPC2 function as a tag team duo to mobilize cholesterol. Proceedings of the National Academy of Sciences of the United States of America 105, 15223-15224.

Tang, M., Gao, G., Rueda, C.B., Yu, H., Thibodeaux, D.N., Awano, T., Engelstad, K.M., SanchezQuintero, M.J., Yang, H., Li, F., et al. (2017). Brain microvasculature defects and Glut1 deficiency syndrome averted by early repletion of the glucose transporter-1 protein. Nature communications 8 , 14152.

Terasaki, M. (2018). Axonal endoplasmic reticulum is very narrow. Journal of cell science 131.

The Lancet, N. (2019). Essential medicines for patients with multiple sclerosis. The Lancet. Neurology $18,1067$.

Theesfeld, C.L., Pourmand, D., Davis, T., Garza, R.M., and Hampton, R.Y. (2011). The sterol-sensing domain (SSD) directly mediates signal-regulated endoplasmic reticulum-associated degradation (ERAD) of 3-hydroxy-3-methylglutaryl (HMG)-CoA reductase isozyme Hmg2. The Journal of biological chemistry 286, 26298-26307.

Thiessen, J.D., Zhang, Y., Zhang, H., Wang, L., Buist, R., Del Bigio, M.R., Kong, J., Li, X.M., and Martin, M. (2013). Quantitative MRI and ultrastructural examination of the cuprizone mouse model of demyelination. NMR in biomedicine 26, 1562-1581.

Thompson, A.J., Baranzini, S.E., Geurts, J., Hemmer, B., and Ciccarelli, O. (2018). Multiple sclerosis. Lancet 391, 1622-1636.

Togha, M., Karvigh, S.A., Nabavi, M., Moghadam, N.B., Harirchian, M.H., Sahraian, M.A., Enzevaei, A., Nourian, A., Ghanaati, H., Firouznia, K., et al. (2010). Simvastatin treatment in patients with relapsing-remitting multiple sclerosis receiving interferon beta 1a: a double-blind randomized controlled trial. Multiple sclerosis 16, 848-854.

Torkildsen, O., Brunborg, L.A., Myhr, K.M., and Bo, L. (2008). The cuprizone model for demyelination. Acta neurologica Scandinavica. Supplementum 188, 72-76.

Torkildsen, O., Myhr, K.M., and Bo, L. (2016). Disease-modifying treatments for multiple sclerosis - a review of approved medications. European journal of neurology 23 Suppl 1, 18-27.

Trommsdorff, M., Gotthardt, M., Hiesberger, T., Shelton, J., Stockinger, W., Nimpf, J., Hammer, R.E., Richardson, J.A., and Herz, J. (1999). Reeler/Disabled-like disruption of neuronal migration in knockout mice lacking the VLDL receptor and ApoE receptor 2. Cell 97, 689-701. 
Umeda, K., Ikenouchi, J., Katahira-Tayama, S., Furuse, K., Sasaki, H., Nakayama, M., Matsui, T., Tsukita, S., Furuse, M., and Tsukita, S. (2006). ZO-1 and ZO-2 independently determine where claudins are polymerized in tight-junction strand formation. Cell 126, 741-754.

Valenza, M., Marullo, M., Di Paolo, E., Cesana, E., Zuccato, C., Biella, G., and Cattaneo, E. (2015). Disruption of astrocyte-neuron cholesterol cross talk affects neuronal function in Huntington's disease. Cell death and differentiation 22, 690-702.

van Deijk, A.F., Camargo, N., Timmerman, J., Heistek, T., Brouwers, J.F., Mogavero, F., Mansvelder, H.D., Smit, A.B., and Verheijen, M.H. (2017). Astrocyte lipid metabolism is critical for synapse development and function in vivo. Glia 65, 670-682.

Van Itallie, C.M., Fanning, A.S., Holmes, J., and Anderson, J.M. (2010). Occludin is required for cytokine-induced regulation of tight junction barriers. Journal of cell science 123, 2844-2852.

Vance, J.E., and Hayashi, H. (2010). Formation and function of apolipoprotein E-containing lipoproteins in the nervous system. Biochimica et biophysica acta 1801, 806-818.

Vance, J.E., Pan, D., Campenot, R.B., Bussiere, M., and Vance, D.E. (1994). Evidence that the major membrane lipids, except cholesterol, are made in axons of cultured rat sympathetic neurons. Journal of neurochemistry $62,329-337$.

Vigano, F., Mobius, W., Gotz, M., and Dimou, L. (2013). Transplantation reveals regional differences in oligodendrocyte differentiation in the adult brain. Nature neuroscience 16, 1370-1372.

Vollmer, T., Key, L., Durkalski, V., Tyor, W., Corboy, J., Markovic-Plese, S., Preiningerova, J., Rizzo, M., and Singh, I. (2004). Oral simvastatin treatment in relapsing-remitting multiple sclerosis. Lancet $363,1607-1608$.

Vorbrodt, A.W., and Dobrogowska, D.H. (2003). Molecular anatomy of intercellular junctions in brain endothelial and epithelial barriers: electron microscopist's view. Brain research. Brain research reviews 42, 221-242.

Voskuhl, R.R., Itoh, N., Tassoni, A., Matsukawa, M.A., Ren, E., Tse, V., Jang, E., Suen, T.T., and Itoh, Y. (2019). Gene expression in oligodendrocytes during remyelination reveals cholesterol homeostasis as a therapeutic target in multiple sclerosis. Proceedings of the National Academy of Sciences of the United States of America 116, 10130-10139.

Wahrle, S.E., Jiang, H., Parsadanian, M., Legleiter, J., Han, X., Fryer, J.D., Kowalewski, T., and Holtzman, D.M. (2004). ABCA1 is required for normal central nervous system ApoE levels and for lipidation of astrocyte-secreted apoE. The Journal of biological chemistry 279, 40987-40993.

Waldner, H., Collins, M., and Kuchroo, V.K. (2004). Activation of antigen-presenting cells by microbial products breaks self tolerance and induces autoimmune disease. The Journal of clinical investigation 113, 990-997.

Walther, T.C., and Farese, R.V., Jr. (2009). The life of lipid droplets. Biochimica et biophysica acta $1791,459-466$.

Walther, T.C., and Farese, R.V., Jr. (2012). Lipid droplets and cellular lipid metabolism. Annual review of biochemistry $81,687-714$.

Wang, D., Li, S.P., Fu, J.S., Zhang, S., Bai, L., and Guo, L. (2016a). Resveratrol defends blood-brain barrier integrity in experimental autoimmune encephalomyelitis mice. Journal of neurophysiology 116 , 2173-2179.

Wang, H., and Eckel, R.H. (2014). What are lipoproteins doing in the brain? Trends in endocrinology and metabolism: TEM 25, 8-14. 
Wang, J., Liu, Y., Liu, F., Huang, C., Han, S., Lv, Y., Liu, C.J., Zhang, S., Qin, Y., Ling, L., et al. (2016b). Loss-of-function Mutation in PMVK Causes Autosomal Dominant Disseminated Superficial Porokeratosis. Scientific reports 6, 24226.

Wang, J., Sui, R.X., Miao, Q., Wang, Q., Song, L.J., Yu, J.Z., Li, Y.H., Xiao, B.G., and Ma, C.G. (2020). Effect of Fasudil on remyelination following cuprizone-induced demyelination. CNS neuroscience \& therapeutics $26,76-89$.

Wang, M., and Casey, P.J. (2016). Protein prenylation: unique fats make their mark on biology. Nature reviews. Molecular cell biology 17, 110-122.

Wang, Y., Jin, S., Sonobe, Y., Cheng, Y., Horiuchi, H., Parajuli, B., Kawanokuchi, J., Mizuno, T., Takeuchi, H., and Suzumura, A. (2014). Interleukin-1beta induces blood-brain barrier disruption by downregulating Sonic hedgehog in astrocytes. PloS one 9, e110024.

Wang, Y., Rogers, P.M., Su, C., Varga, G., Stayrook, K.R., and Burris, T.P. (2008). Regulation of cholesterologenesis by the oxysterol receptor, LXRalpha. The Journal of biological chemistry 283, 26332-26339.

Wang, Z., Sadovnick, A.D., Traboulsee, A.L., Ross, J.P., Bernales, C.Q., Encarnacion, M., Yee, I.M., de Lemos, M., Greenwood, T., Lee, J.D., et al. (2016c). Nuclear Receptor NR1H3 in Familial Multiple Sclerosis. Neuron 92, 555.

Wimmer, I., Tietz, S., Nishihara, H., Deutsch, U., Sallusto, F., Gosselet, F., Lyck, R., Muller, W.A., Lassmann, H., and Engelhardt, B. (2019). PECAM-1 Stabilizes Blood-Brain Barrier Integrity and Favors Paracellular T-Cell Diapedesis Across the Blood-Brain Barrier During Neuroinflammation. Frontiers in immunology 10, 711.

Wolburg-Buchholz, K., Mack, A.F., Steiner, E., Pfeiffer, F., Engelhardt, B., and Wolburg, H. (2009). Loss of astrocyte polarity marks blood-brain barrier impairment during experimental autoimmune encephalomyelitis. Acta neuropathologica 118, 219-233.

Wosik, K., Cayrol, R., Dodelet-Devillers, A., Berthelet, F., Bernard, M., Moumdjian, R., Bouthillier, A., Reudelhuber, T.L., and Prat, A. (2007). Angiotensin II controls occludin function and is required for blood brain barrier maintenance: relevance to multiple sclerosis. The Journal of neuroscience : the official journal of the Society for Neuroscience 27, 9032-9042.

Xie, C., Lund, E.G., Turley, S.D., Russell, D.W., and Dietschy, J.M. (2003). Quantitation of two pathways for cholesterol excretion from the brain in normal mice and mice with neurodegeneration. Journal of lipid research $44,1780-1789$.

Xu, Q., Bernardo, A., Walker, D., Kanegawa, T., Mahley, R.W., and Huang, Y. (2006). Profile and regulation of apolipoprotein $E$ (ApoE) expression in the CNS in mice with targeting of green fluorescent protein gene to the ApoE locus. The Journal of neuroscience : the official journal of the Society for Neuroscience 26, 4985-4994.

Xu, S., Zhang, X., and Liu, P. (2018). Lipid droplet proteins and metabolic diseases. Biochimica et biophysica acta. Molecular basis of disease 1864, 1968-1983.

Yamasaki, R., Lu, H., Butovsky, O., Ohno, N., Rietsch, A.M., Cialic, R., Wu, P.M., Doykan, C.E., Lin, J., Cotleur, A.C., et al. (2014). Differential roles of microglia and monocytes in the inflamed central nervous system. The Journal of experimental medicine 211, 1533-1549.

Yang, C., McDonald, J.G., Patel, A., Zhang, Y., Umetani, M., Xu, F., Westover, E.J., Covey, D.F., Mangelsdorf, D.J., Cohen, J.C., et al. (2006). Sterol intermediates from cholesterol biosynthetic pathway as liver X receptor ligands. The Journal of biological chemistry 281, 27816-27826.

Yao, Y., Chen, Z.L., Norris, E.H., and Strickland, S. (2014). Astrocytic laminin regulates pericyte differentiation and maintains blood brain barrier integrity. Nature communications 5, 3413. 
Yazdi, A., Baharvand, H., and Javan, M. (2015). Enhanced remyelination following lysolecithininduced demyelination in mice under treatment with fingolimod (FTY720). Neuroscience 311, 34-44.

Ye, J., and DeBose-Boyd, R.A. (2011). Regulation of cholesterol and fatty acid synthesis. Cold Spring Harbor perspectives in biology 3 .

Yeung, M.S.Y., Djelloul, M., Steiner, E., Bernard, S., Salehpour, M., Possnert, G., Brundin, L., and Frisen, J. (2019). Dynamics of oligodendrocyte generation in multiple sclerosis. Nature 566, 538-542.

Ying, Z., Zhai, R., McLean, N.A., Johnston, J.M., Misra, V., and Verge, V.M. (2015). The Unfolded Protein Response and Cholesterol Biosynthesis Link Luman/CREB3 to Regenerative Axon Growth in Sensory Neurons. The Journal of neuroscience : the official journal of the Society for Neuroscience 35, 14557-14570.

Zeis, T., Howell, O.W., Reynolds, R., and Schaeren-Wiemers, N. (2018). Molecular pathology of Multiple Sclerosis lesions reveals a heterogeneous expression pattern of genes involved in oligodendrogliogenesis. Experimental neurology 305, 76-88.

Zelcer, N., Hong, C., Boyadjian, R., and Tontonoz, P. (2009). LXR regulates cholesterol uptake through Idol-dependent ubiquitination of the LDL receptor. Science 325, 100-104.

Zhang, Y., Chen, K., Sloan, S.A., Bennett, M.L., Scholze, A.R., O'Keeffe, S., Phatnani, H.P., Guarnieri, P., Caneda, C., Ruderisch, N., et al. (2014). An RNA-sequencing transcriptome and splicing database of glia, neurons, and vascular cells of the cerebral cortex. The Journal of neuroscience : the official journal of the Society for Neuroscience 34, 11929-11947.

Zhao, S., Hu, X., Park, J., Zhu, Y., Zhu, Q., Li, H., Luo, C., Han, R., Cooper, N., and Qiu, M. (2007). Selective expression of LDLR and VLDLR in myelinating oligodendrocytes. Developmental dynamics : an official publication of the American Association of Anatomists 236, 2708-2712.

Zhou, Y., Wang, Y., Tischfield, M., Williams, J., Smallwood, P.M., Rattner, A., Taketo, M.M., and Nathans, J. (2014). Canonical WNT signaling components in vascular development and barrier formation. The Journal of clinical investigation 124, 3825-3846.

Zorzella-Pezavento, S.F., Chiuso-Minicucci, F., Franca, T.G., Ishikawa, L.L., da Rosa, L.C., Marques, C., Ikoma, M.R., and Sartori, A. (2013). Persistent inflammation in the CNS during chronic EAE despite local absence of IL-17 production. Mediators of inflammation 2013, 519627. 


\section{Acknowledgements}

Firstly, I would like to express my sincere gratitude to my advisor Dr. Gesine Saher for her continuous support and giving me the opportunity to work freely on these challenging projects. I am deeply thankful for inspiring scientific discussions, the opportunity to plan and execute own scientific ideas, advises for my scientific carrier and sharing important aspects of scientific profession.

I extend by special thanks to Prof. Klaus-Armin Nave for his respect, critical advises and for sharing his scientific experience in addition to support me in sharing my findings with the scientific community.

I also would like to thank Prof. Dr. Christine Stadelmann-Nessler as part of my thesis committee and who agreed on reviewing my thesis, for her insightful comments and encouragement, but also her briefing and advises with regards to human Multiple Sclerosis histopathology.

I further thank Prof. Dr. Alexander Flügel for his critical advises as part of my thesis committee.

Besides my advisors, I would like to thank my thesis examination committee: Prof. Dr. Dr. Hannelore Ehrenreich, Prof. Dr. Tiago Fleming Outeiro and Prof. Dr. Till Ischebeck.

I am sincerely thankful to Dr. Julia Edgar, which gave me the opportunity to perform experiments independently at an early stage of my scientific education and for her advises during collaborative projects.

My exceptional gratitude goes to Dr. Dr. Francesca Odoardi for her mentorship during early scientific education and advises during collaborative work.

I want to extend by thankfulness to Dr. Benoit Barrette and Dr. Iva Tzvetanova for their scientific advises and encouraging support.

I also thank Prof. Dr. Mikael Simons for his scientific advises and collaborative contribution to my projects.

I am thankful to my collaborators, Ph.D. Ludovico Cantuti-Castelvetri, Dr. Ruth Stassart, Dr. David Liebetanz, Dr. Payam Dibaj Dr. Kathrin Kusch and Dr. David Ewers and Leon Hosang for their supportive contribution towards my project. I would like to emphasize my appreciation to Leon Hosang for his inspiring motivation and for making the impossible possible. 
I want to express my deepest gratitude to my colleagues and friends.

\section{From the Saher group:}

Jan Winchenbach for scientific debates, his contributions to these projects and the first beer in Göttingen.

Sina Stumpf and Tim Duking for valuable scientific discussions throughout the years.

Nina Gerndt for her essential technical assistance and contributions to these projects.

Lena Spieth for her contribution to this project during her lab rotation and master thesis. It was a privilege to supervise her.

Former students of mine, Lennart Schlaphoff and Jonathan Neuber, for their contributions to these projects during their lab rotation and/or master thesis.

Carolin Böhler and Silvia Thüne for valuable technical assistance.

\section{and beyond:}

Constanze Depp for inspiring discussion and productive arguments leading to new scientific hypotheses.

Maria Eichel and Martin Meschkat for their advises regarding visualization and presentation of scientific data.

Ting Sun for her advises and contribution to single cell RNAseq. datasets.

Ulli Bode, Ramona Jung, Annette Fahrenholz and Verena Meywirth for very valuable technical support.

Dr. Wiebke Möbius, Torben Ruhwedel and Boguslawa Sadowski for advices and introductions into electron microscopy. My particular thanks goes to Torben Ruhwedel for his inspiring and encouraging motivation.

I thank Tanja Pawelz, Ines Malade, Cornelia Casper, Nadja Hoffmeister, Bianca Nickel and Dr. Ursula Fünfschilling for providing an excellent mouse house and transgenic animal facility.

I am thankful to Gabriele Endo and Michaela Schmalstieg not only for their support with administrative issues, but also for their lovely care and positive spirit.

At last but not least I thank my family for their love and care, and for always reminding me that there is also a different aspect of life besides science.

And above all, I thank my wife who did not give up in trying to understand all ridiculous scientific ideas I had, with her words: "Behind every successful man there is a strong woman" 


\section{Publications and presentations}

\section{Publications}

Winchenbach, J., Duking, T., Berghoff, S.A., Stumpf, S.K., Hulsmann, S., Nave, K.A., and Saher, G. (2016). Inducible targeting of CNS astrocytes in Aldh1/1CreERT2 BAC transgenic mice.

F1000Research 5, 2934.

Berghoff, S.A., Gerndt, N., Winchenbach, J., Stumpf, S.K., Hosang, L., Odoardi, F., Ruhwedel, T., Böhler, C., Barrette, B., Stassart, R., et al. (2017b). Dietary cholesterol promotes repair of demyelinated lesions in the adult brain.

Nature communications 8, 14241.

Berghoff, S.A., Duking, T., Spieth, L., Winchenbach, J., Stumpf, S.K., Gerndt, N., Kusch, K., Ruhwedel, T., Mobius, W., and Saher, G. (2017a). Blood-brain barrier hyperpermeability precedes demyelination in the cuprizone model.

Acta neuropathologica communications 5, 94.

Pan H., Oliveira B., Saher G., Dere E., Tapken D., Mitjans M., Seidel J., Wesolowski J., Wakhloo D., Klein-Schmidt C., Ronnenberg A., Schwabe K., Trippe R., MätzRensing K., Berghoff S., Al-Krinawe Y., Martens H., Begemann M., Stöcker W., Kaup F.J., Mischke R., Boretius S., Nave K.A., Krauss J.K., Hollmann M, Lühder F. \& Ehrenreich H. (2019). Uncoupling the widespread occurrence of anti-NMDAR1 autoantibodies from neuropsychiatric disease in a novel autoimmune model.

Mol Psychiatry 24, 1489-1501.

Stumpf, S.K., Berghoff, S.A., Trevisiol, A., Spieth, L., Duking, T., Schneider, L.V., Schlaphoff, L., Dreha-Kulaczewski, S., Bley, A., Burfeind, D., et al. (2019). Ketogenic diet ameliorates axonal defects and promotes myelination in Pelizaeus-Merzbacher disease.

Acta neuropathologica 138, 147-161.

Lena Spieth, Stefan A. Berghoff, Sina K. Stumpf, Jan Winchenbach, Takashi Watanabe, Nina Gerndt, Tim Düking, Thomas Michaelis, Sabine Hofer, Jens Frahm2, Katrin Willig, Petra Hülper and Gesine Saher (2019), Simultaneous exposure to anesthesia and cytostatic improves therapy of glioblastoma in mice.

(Submitted Manuscript)

Berghoff S.A., Spieth L., Sun T., Schlaphoff L., Hosang L. , Depp C., Düking T., Winchenbach J., Neuber J, Ewers D., Scholz P., Paap F., Cantuti-Castelvetri L., Sasmita A.O., Meschkat M., Ruhwedel T., Möbius W. , Odoardi F., Ischebeck T., Edgar J.M. , Prinz M, Sankowski R., Stadelmann-Nessler C., Simons M, Nave K.A., and Saher G. Phase and cell-type specific post-squalene sterol synthesis coordinates inflammation and remyelination

(Prepared Manuscript) 


\section{Poster presentations}

October 2018

Berlin, Germany

March 2019

Kassel, Germany

June 2019

Keysone, CA, USA
ECTRIMS

$34^{\text {th }}$ Congress of European Committee for Treatment

and Research in Multiple Sclerosis

P741

Title: Cholesterol supports repair in mouse models of demyelination and remyelination

Current Topics in Myelin Research

Titel: Cholesterol supports repair in mouse models of demyelination and remyelination

Keystone Symposia

Neural Enviroment in Disease:

Glial Responses and Neuroinflammation

Titel: Cell-type specific cholesterol synthesis coordinates inflammation and remyelination in demyelinated lesions

Oligo meeting

Title: Cell-type specific cholesterol synthesis during remyelination

Keystone Symposia

Title: Cell-type specific cholesterol synthesis coordinates inflammation and remyelination in demyelinated lesions 


\section{Own contribution}

Publication 1: Dietary cholesterol promotes repair of demyelinated lesions in the adult brain

Stefan A. Berghoff, Nina Gerndt, Jan Winchenbach, Sina K. Stumpf, Leon Hosang, Francesca Odoardi, Torben Ruhwedel, Carolin Böhler, Benoit Barrette, Ruth Stassart, David Liebetanz, Payam Dibaj, Wiebke Möbius, Julia M. Edgar \& Gesine Saher

Published: 24 Jan 2017

G.S., S.A.B., J.M.E., D.L. and W.M. participated in the planning and designing the experiments. S.A.B. was involved in performing all experiments. N.G. and J.M.E.

Author contribution: performed cell culture experiments, S.K.S., P.D. and J.W., characterized the mouse pathologies, C.B. performed expression analyses, B.B. was involved in EAE experiments, L.H. and F.O. did flow cytometry with subsequent expression analyses, T.R. performed electron microscopy analysis, W.M. edited the manuscript, S.A.B., J.M.E. and G.S. wrote the manuscript.

Own contribution

a) Feeding and EAE induction

b) Bodyweight monitoring

Figure $1 \quad$ c) Feeding, Perfusion, sectioning, histological staining, imaging and quantification LFB-PAS staining was done by collaborators

a) Evans blue detection (Histology)

b) Evans Blue extravasation assay

EAE Evans Blue analysis was done by Lennart Wiegand

c) Bodipy-cholesterol injection

Imaging was performed by Gesine Saher and Payam Dibaj

d) Bodipy-cholesterol extraction assay

a) Gesine Saher

b) Feeding, Perfusion, sectioning, histological staining, imaging and

Figure 3 quantification

c) Feeding, Perfusion, sectioning, histological staining, imaging and quantification

a) Gesine Saher

b) Feeding, Perfusion, sectioning, histological staining, imaging and quantification

Figure $4 \quad$ c) Perfusion, imaging and quantification

d) Fectioning and contrasting was performed by EM facility quantification

Figure 5 a) Gesine Saher

b) Feeding, Perfusion, sectioning, histological staining, imaging and quantification 
Table continued

c) Feeding, Perfusion, sectioning, histological staining, imaging and quantification

d) Feeding, Perfusion, sectioning, histological staining, imaging and quantification

e) Perfusion, imaging and quantification

Sectioning and contrasting was performed by EM facility

f) Feeding, Perfusion, sectioning, histological staining, imaging and

quantification

g) Feeding, quantification

Figure 6

a) Feeding, Perfusion, histological staining, imaging and quantification Injection were done by Ruth Stassart

b) Feeding, Perfusion and quantification

Sectioning, contrasting and imaging was performed by EM facility

c) Quantification

d) Bodyweight monitoring and Quantification

a) Nina Gerndt and Gesine Saher

b) Cell culture, staining and analysis

c) QPCR and quantification

d) QPCR and quantification

e) QPCR and quantification

Figure $7 \quad$ f) $\quad$ qPCR and quantification

g) qPCR and quantification

h) QPCR and quantification

i) Nina Gerndt and Gesine Saher

j) Nina Gerndt and Gesine Saher

k) qPCR and quantification

RNA isolation was performed by Carolin Böhler

Figure $8 \quad$ Stefan Berghoff and Gesine Saher

\footnotetext{
a) Feeding and EAE induction

Supplementary

Figure 1

b) Feeding, Perfusion, histological staining, imaging and quantification

c) Tissue isolation

FACS analysis was performed by collaborators
}

a) Feeding and EAE induction

Supplementary

b) Tissue isolation

Figure 2

FACS analysis was performed by collaborators

c) $\quad$ QPCR and quantification was performed by Leon Hosang

Supplementary

Figure 3

a-c)

Feeding and serum collection 
Table continued

$\begin{array}{cll} & \text { a) } & \text { Feeding, Perfusion and imaging } \\ \text { Supplementary } & \text { b) } & \text { Feeding, Perfusion and imaging } \\ \text { Figure } 4 & \text { c) } & \text { Quantification } \\ & & \text { Sectioning and contrasting was performed by EM facility }\end{array}$

Supplementary Figure 5

a) Bodyweight, monitoring and Quantification

Supplementary

a) Feeding, Perfusion, histological staining, imaging and quantification Figure 6

Supplementary a-c) Monitoring and Quantification

Figure 7

Supplementary Figure 8
a) Nina Gerndt and Gesine Saher

b) Cell culture, staining and imaging

Supplementary Table 1

qPCR and quantification

Supplementary

Table 2

QPCR and quantification

Supplementary

Table 3

Primer design and validation

Supplementary Table 4

Imaging characteristics 

$\begin{array}{ll}\text { Publication 2: } & \begin{array}{l}\text { Blood-brain barrier hyperpermeability precedes demyelination in the cuprizone } \\ \text { model }\end{array}\end{array}$

Authors: $\quad$ Stefan A. Berghoff, Tim Düking, Lena Spieth, Jan Winchenbach, Sina K. Stumpf, Nina Gerndt, Kathrin Kusch, Torben Ruhwedel, Wiebke Möbius and Gesine Saher

Published: $\quad 01$ December 2017

Author contribution:

GS, and SAB conceptualized the study and designed the experiments, SAB, TD, JW, SKS, NG, LS, TR, BHC, WM performed experiments. KK generated the GLUT1 antibody. SAB and GS wrote the manuscript. All authors read and approved the final manuscript.

Own contribution

a) Animal treatment, tissue isolation, qPCR and quantification

b) Animal treatment, tissue isolation, qPCR and quantification

c) Animal treatment, perfusion, imaging and quantification

Sectioning and contrasting was performed by EM facility

d) Animal treatment, perfusion, staining and imaging

Figure 1

e) Animal treatment, perfusion, staining and imaging

Imaging was done with help of Jan Winchenbach

f) Gesine Saher, Jan Winchenbach

g) Quantification

h) Perfusion, staining, imaging and quantification

e) Cell culture and quantification

f) Nina Gerndt

Figure 2

g) Cell culture, staining, imaging and quantification

Together with Gesine Saher

h) Cell culture, qPCR and quantification

Cell culture together with Nina Gerndt

d) Animal treatment, perfusion, staining and imaging Imaging was done with help of Jan Winchenbach

e) Quantification

Figure 3

f) Animal treatment and tissue isolation Western Blot by Lena Spieth

g) Animal treatment, perfusion, staining, imaging and quantification

h) Animal treatment, perfusion, staining and imaging

i) Quantification

e) Gesine Saher and Stefan Berghoff

b-g) Experimental procedure and quantification

Figure 4 h) Animal treatment, tissue isolation qPCR and quantification

i) Animal treatment, tissue isolation qPCR and quantification RNA isolation and cDNA synthesis by Lena Spieth 
Table continued

a-c) Animal treatment, tissue isolation, qPCR and quantification

Figure 5 RNA isolation and CDNA synthesis by Lena Spieth

d-f) Experimental procedure and quantification

j) Animal treatment, tissue isolation, qPCR and quantification Cell isolation by Tim Düking

Supplementary a) Animal treatment, perfusion, staining and imaging

Figure 1

b) Quantification

Supplementary

Figure 2

a-c) Animal treatment, perfusion, imaging and quantification Sectioning and contrasting was performed by EM facility Scheme: Gesine Saher

a-d) Nina Gerndt performed experiments

i) $\quad$ QPCR and quantification

Supplementary

Figure 3

Cell culture experiments by Nina Gerndt

j) Cell culture, qPCR and quantification

k) Cell culture, qPCR and quantification

Cells were provided by Dept. Clinical Neuroscience

Supplementary

Figure 4

Animal treatment, perfusion, staining and imaging 
Manuscript/ Publication 3:

Authors: contribution:

Figure 2
A) Scheme design
B) Animal treatment, perfusion, staining and imaging
C) Scheme design
D) Scheme design
E) Cell isolation qPCR and quantification
F) Animal treatment, tissue isolation $\mathrm{QPCR}$ and quantification
G) $\mathrm{QPCR}$ and quantification
Cell isolation was performed by Tim Düking and Lena Spieth
$\mathrm{H}$ ) Animal treatment, perfusion, staining and imaging

Figure 1
A) Scheme design
B) Animal treatment, perfusion, staining and imaging
C) Animal treatment, tissue isolation, qPCR and quantification
D) Cell culture experiment performed by Lennart Schlaphoff
E) Animal treatment, perfusion, staining and imaging
F) Animal treatment, perfusion, staining and imaging

G) Animal treatment and tissue isolation

Western Blot was performed by Jonathan Neuber

H) $\quad$ qPCR and quantification Cell isolation was performed by Lena Spieth

I) Cell culture experiment performed by Lennart Schlaphoff

J) Animal treatment, perfusion, staining and imaging

K) Lysolecithin induction was performed by Ludovico Cantuti-Castelvetri

K) $\quad$ EAE induction and tissue isolation Scoring was performed by Lena Spieth FACS analysis was performed by Leon Hosang

A) Scheme design

Figure 3

B) EAE induction and tissue isolation

C) EAE induction and tissue isolation FACS analysis was performed by Leon Hosang 
Table continued

D) $\quad E A E$ induction and tissue isolation Scoring was performed by Lena Spieth

E) EAE induction and tissue isolation

Figure 3

$\mathrm{F}$ ) Animal treatment, tissue isolation $\mathrm{QPCR}$ and quantification

G) $\quad$ QPCR and quantification

$\mathrm{H}) \quad$ Analysis

Figure 4

A-H) $\quad$ qPCR and quantification

Cell culture experiment performed by Lennart Schlaphoff

I-J) Samples preparation and lyophilization

Lipid Mass spectrometry performed by Till Ischebeck

K) $\quad$ qPCR and quantification

Cell culture experiment performed by Lennart Schlaphoff

L) Scheme design

A-D) Cuprizone treatment /EAE induction, $\mathrm{QPCR}$ and quantification Cell isolation was performed by Lena Spieth Lysolecithin induction was performed by Ludovico Cantuti-Castelvetri $M B P C K O$ induction performed by Martin Meschkat

E) Scheme design

F) Cuprizone treatment /EAE induction, tissue isolation and lyophilization

Figure 5

G) Cuprizone treatment, sample preparation and lyophilization

Cell isolation was performed by Lena Spieth

H) Single cell human RNAseq. reanalysis together with Ting Sun

I) Samples preparation, imaging and lyophilization Histopathological classification and sample preparation by Christine Stadelmann-Nessler and Franziska Paap

Lipid Mass spectrometry performed by Till Ischebeck

A) Imaging and quantification

Cell culture experiment performed by Julia M. Edgar

B) Lnimal treatment, perfusion, staining and imaging
Lysolecithin induction was performed by Ludovico Cantuti-Castelvetri

Figure $6 \quad$ C) Scoring was performed by Lena Spieth

D) EAE induction, treatment and tissue isolation

FACS analysis was performed by Leon Hosang

E) qPCR and quantification

Cell isolation was performed by Lena Spieth

F) Scheme design 
Table continued

\begin{tabular}{|c|c|c|}
\hline \multirow{7}{*}{$\begin{array}{l}\text { Supplementary } \\
\quad \text { Figure } 1\end{array}$} & $\begin{array}{l}\text { A) } \\
\text { B) }\end{array}$ & $\begin{array}{l}\text { Cuprizone treatment, tissue isolation, } \mathrm{QPCR} \text { and quantification } \\
\text { qPCR and quantification } \\
\text { Cell isolation was performed by Lena Spieth }\end{array}$ \\
\hline & C) & Scheme design, qPCR and quantification \\
\hline & & Cell isolation was performed by Tim Düking and Lena Spieth \\
\hline & D) & $\begin{array}{l}\text { Bodyweight monitoring, blood collection, serum preparation and } \\
\text { quantification }\end{array}$ \\
\hline & & Serum cholesterol was measured by UMG Göttigen (Zentrallabor) \\
\hline & E) & Scheme design \\
\hline & F) & $\begin{array}{l}\text { Cuprizone treatment, perfusion, histological staining, imaging and } \\
\text { quantification }\end{array}$ \\
\hline
\end{tabular}

A) EAE induction, $\mathrm{qPCR}$ and quantification

Cell isolation was performed by Lena Spieth

B) Scheme design, qPCR and quantification MBPCKO induction performed by Martin Meschkat

Supplementary

Cell isolation was performed by Lena Spieth

Figure 2

C) Bodyweight monitoring, blood collection, serum preparation, qPCR and

D) Cuprizone treatment, perfusion, histological staining, imaging and quantification

Together with Jan Winchenbach

E) Beam testing and quantification

Together with Lena Spieth

A) Scheme design

B) Cuprizone treatment, perfusion and imaging

Sectioning and contrasting was performed by EM facility

Cell culture experiment performed by Lennart Schlaphoff

D) $\quad$ QPCR and quantification

Cell culture experiment performed by Lennart Schlaphoff

E) Cell culture experiment performed by Lennart Schlaphoff

Supplementary F) Cuprizone treatment, perfusion, histological staining, imaging and

quantification

G) Cuprizone treatment, perfusion and imaging

Sectioning and contrasting was performed by EM facility

H) QPCR and quantification

Cell culture experiment performed by Lennart Schlaphoff

I) Perfusion, histological staining, imaging and quantification

Lysolecithin induction was performed by Ludovico Cantuti-Castelvetri

J) Scheme design

K) EAE induction, treatment and tissue isolation

FACS analysis was performed by Leon Hosang

A) EAE induction and treatment

Scoring was performed by Lena Spieth

FACS analysis was performed by Leon Hosang

Supplementary

B-C) EAE induction, blood collection, serum preparation and quantification

Figure 4

D) EAE induction, blood collection, serum preparation and quantification

Serum cholesterol was measured by UMG Göttigen (Zentrallabor)

E) EAE induction, blood collection, serum preparation

Squalene measurement performed by David Ewers 
Table continued

\begin{tabular}{|c|c|c|}
\hline \multirow{6}{*}{$\begin{array}{l}\text { Supplementary } \\
\text { Figure } 4\end{array}$} & F) & Scheme design \\
\hline & G) & Cuprizone treatment,serum preparation and quantification \\
\hline & & Serum cholesterol was measured by UMG Göttigen (Zentrallabor) \\
\hline & H) & Cuprizone treatment, blood collection, serum preparation \\
\hline & & Squalene measurement performed by David Ewers \\
\hline & I) & Cuprizone treatment, perfusion, staining, imaging and quantification \\
\hline \multirow{5}{*}{$\begin{array}{l}\text { Supplementary } \\
\text { Figure } 5\end{array}$} & A-E) & QPCR and quantification \\
\hline & & Cell culture experiment performed by Lennart Schlaphoff \\
\hline & $\begin{array}{l}\mathrm{H}-\mathrm{G}) \\
\mathrm{H}-\mathrm{I})\end{array}$ & $\begin{array}{l}\text { Cell culture experıment performed by Lennart Schlaphott } \\
\text { qPCR and quantification }\end{array}$ \\
\hline & & Cell culture experiment performed by Lennart Schlaphoff \\
\hline & $\mathrm{J}-\mathrm{K})$ & Cell culture experiment performed by Lennart Schlaphoff \\
\hline
\end{tabular}

A) Cuprizone treatment, tissue preparation and lyophilization Cell isolation was performed by Lena Spieth

B) Data provided from Mikael Simons

C) Cuprizone treatment, tissue isolation and lyophilization

Supplementary

Dipid Mass spectrometry performed by Till Ischebeck

Figure 6

D) EAE induction, tissue isolation and lyophilization

E) LAE induction, QPCR and quantification

Cell isolation was performed by Lena Spieth

Lysolecithin induction was performed by Ludovico Cantuti-Castelvetri

$M B P C K O$ induction performed by Martin Meschkat

F) EAE induction, tissue isolation and lyophilization

Lipid Mass spectrometry performed by Patricia Scholz

A-D) Single cell human RNAseq. reanalysis together with Ting Sun

Supplementary

E-G) Samples preparation, imaging and lyophilization

Figure 7

Histopathological classification and sample preparation by

Christine Stadelmann-Nessler and Franziska Paap

Lipid Mass spectrometry performed by Till Ischebeck

A) Animal treatment, blood collection, serum preparation and quantification

A) Serum cholesterol was measured by UMG Göttigen (Zentrallabor)

B) Lysolecithin induction was performed by Ludovico Cantuti-Castelvetri

B) Animal treatment, blood collection, serum preparation

C) Squalene measurement performed by David Ewers

C) EAE induction and treatment

Supplementary

D) Scoring was performed by Lena Spieth

Figure 8

E) EAE induction, tissue isolation, qPCR and quantification

F) EAE induction and treatment

F) Scoring was performed by Jan Winchenbach

Tissue isolation

G-I) FACS analysis was performed by Leon Hosang

qPCR analysis was performed by Leon Hosang

EAE induction, treatment, qPCR and quantification

Cell isolation was performed by Lena Spieth 